\title{
Metonymy in Mind, Language, and Communication
}

\author{
By
}

\section{Xitao Fu}

\begin{abstract}
A thesis
submitted to the Victoria University of Wellington

in fulfilment of the requirements for the degree of

Doctor of Philosophy

in Linguistics
\end{abstract}

Victoria University of Wellington 


\begin{abstract}
The typical view considers metonymy as an intra-domain mapping which involves the source providing mental access to the target within the domain, with PARTWHOLE as the prototypical relation. This commonly held view of metonymy in Cognitive Linguistics pays attention to what happens after the domain, or rather, the WHOLE, has been established. How the WHOLE is formed seems to be missing. Based on the research results of cognitive science, especially in cognitive psychology, developmental psychology, and neuroscience, metonymy is tentatively argued to be an innate cognitive mechanism involving PART-WHOLE FORMING, PART-WHOLE/PART RELATING processes. The PART-WHOLE FORMING process establishes the WHOLE from the PART: It picks up some prominent element(s) in an interactive process to form a patterned experience, and the PARTWHOLE/PART RELATING process relates PART to PART, relating PART to the WHOLE and vice versa. The PART-WHOLE/PART RELATING process is made possible by the PART-WHOLE FORMING process. Metonymic operations usually precede metaphoric operation. Metaphor is essentially grounded on metonymy.
\end{abstract}

Metonymy as a cognitive mechanism is most noticeably realized in language. It operates in various aspects of language and language use. The experience pattern (i.e. the WHOLE) formulated through the PART-WHOLE FORMING process is found to underpin the process of grammaticalization, the development of meaning prototype, and to motivate such daily language use as football nicknaming and to bring in certain cognitive and communicative functions. In the light of this view of metonymy, grammaticalization is considered from the conceptual perspective as a process from the general/global to the specific/local, or from focus on one specific aspect to focus on another particular aspect within the global WHOLE, rather than the usually held concrete-to-abstract process. This also applies to word meaning prototypes. Word meaning develops due to the dynamic of meaning prototypes. When considered from the conceptual perspective, meaning prototypes generally develop from the general/global to the specific/local with the change and specification of contextual situations. The cognitive analysis of football nicknames also suggests that metonymy is overwhelming and provides the requisite basis for metaphor. 
The PART-WHOLE FORMING and the PART-WHOLE/PART RELATING processes of metonymy as an inherent cognitive mechanism often interact in the mind, which is evidenced in language and may be best illustrated through analysis of interactive communication in general, and dialogic discourse in particular. Metonymy in interaction is embodied in its functions and operations in dialogue and its contribution to the dialogue as a discourse entity. Metonymy operates in dialogic discourse in various patterns of GENERAL-SPECIFIC scheme. It operates in the development of dialogue and helps structure the dialogic discourse, making it a coherent discourse entity; it makes meaning out of the local utterance and relates it to the whole dialogue; it underlies the decision-making process, helping make a final decision among alternatives. It also motivates the problem-solving process, helping formulate and organise replies to the questions posed by the counterpart in dialogic discourse, and facilitating the solution of daily problems. 


\section{Acknowledgements}

It would be impossible to have gone through my $\mathrm{PhD}$ research without generous help from others. I would like to acknowledge some people upon the completion of my dissertation.

I am extremely indebted to my supervisors Derek Wallace and Sky Marsen. They have provided me with great support and encouragement in both my research and my life. I always remember the first day we arrived in Wellington. Derek picked us up at the airport and drove us around Wellington City. He and his wife Deborah Battell accommodated us for the first three days and drove us around to help us look for a proper flat, and even introduced a Chinese family to us, hoping we would not feel lonely and could settle down easily. I really appreciate their kindness and hospitability and what they have done for us during these three years. I also remember the first time I went to Derek's Office after the enrolment; he gave me a file box which was filled with sheets of paper: Some printed papers, some printed reference data, and some books that he thought might be of use to my research topic. I was deeply impressed by his considerateness and advance preparation. Sky is also much concerned about my work, offering research help and information, asking after my family frequently. Every time I presented my writing to Derek and Sky, they always read it through with care and patience, giving me valuable comments and heartening encouragement, providing me with information and other materials related to my research. Discussions with them are beneficial and inspirational. Their careful supervision and patient guidance bolster me and help keep me right on track till the end.

I also owe a lot to some well-known scholars in metonymy research and in the scholarship of cognitive science before and during my $\mathrm{PhD}$ research. I feel indebted to Brigitte Nerlich and Beatrice Warren. Well before I came to New Zealand, I had been in frequent contact with Brigitte and benefited quite a lot from her through personal communication and reading her writings. Beatrice Warren sent me her research papers and a book on metonymy when I just became interested in metonymy. I am much obliged to Jean Mandler, Dirk Geeraerts, Antonio Barcelona for both invaluable personal communications and the papers and books they sent me, 
and to Francisco Ruiz de Mendoza, Lesley Stirling for sending me their works on metonymy, and Serge Sharoff for sending me his papers on Sketch Engine Corpus. My thanks also go to Andrew LaVelle and Carolyn Wilshire for beneficial discussions and Gerald Nelson for free access to ICE-Singapore, ICE-Canada, and ICE-Hong Kong during my pilot study of data analysis. I am also grateful to such English football clubs as Watford FC, Cheltenham Town FC, Barnet FC, Cardiff City FC, Brentford FC, Wigan Athletic FC, for their help with football nicknames, and to those authors whose wonderful works I have referred to in my research.

I am thankful to the LALS staff and $\mathrm{PhD}$ fellows for their support. I really appreciate the help from Bernadette Vine and Janet Holmes, who gave me access to ICE-NZ and WSC for my research analysis. My thanks also go to the LALS thesis group for its platform of presentation practice and research exchange.

I thank the VUW Library staff, especially our subject librarian Tony Quinn, for their professional assistance with the requested national and overseas inter-loans and the suggested purchase of some books and theses.

I would like to express my gratitude to the examiners Antonio Barcelona, John Taylor, and Frank Boers for their engagement with my thesis and their constructive and inspirational comments.

My thanks also go to Tony Angelo, Gary Stevens, Shaun Manning, Amanda Golding, and Derek Wallace, and Sky Marsen for their editing and proofreading help.

Finally my gratitude goes to my wife Jane and my daughter Lily. It is their love, support, and encouragement that walk me through the ups and downs. 


\section{Contents}

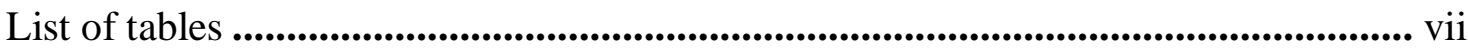

List of figures........................................................................................................................ viii

Chapter 1 Introduction .........................................................................................1

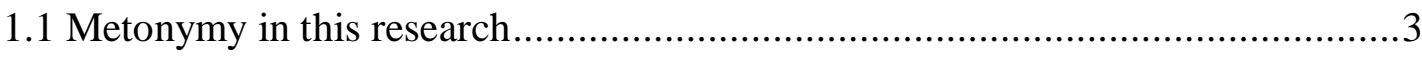

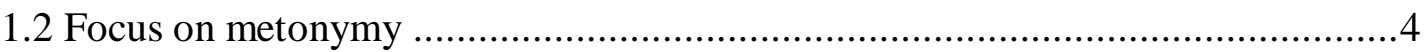

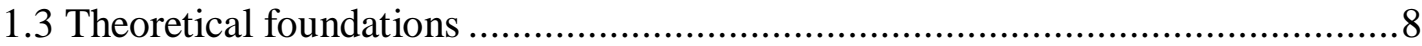

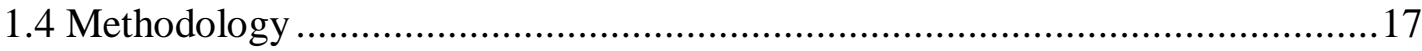

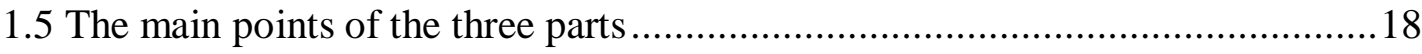

\section{PART ONE METONYMY IN THE MIND}

Chapter 2 Metonymy in the Cognitive Perspective ......................................................227

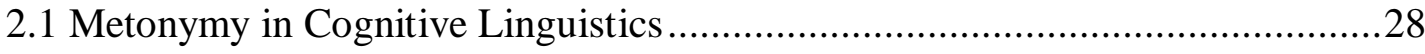

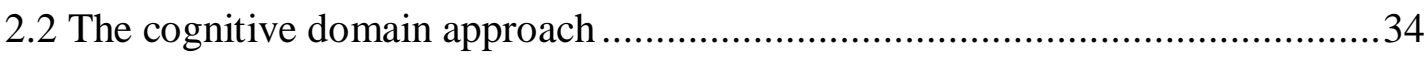

2.3 Interpreting “contiguity" .........................................................................4

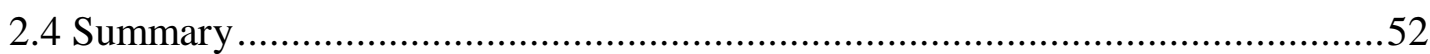

Chapter 3 Metonymy as an Innate Cognitive Mechanism ....................................55

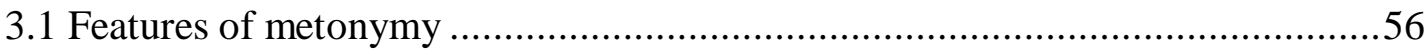

3.2 Metonymy as an inherent cognitive mechanism..........................................60

3.3 Spatial to temporal: PART-PART relating ............................................. 73

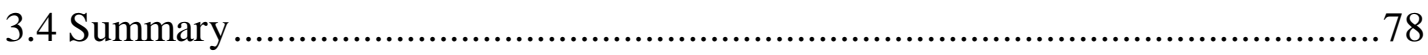

Chapter 4 Grounding Metaphor on Metonymy......................................................81

4.1 Metonymy versus metaphor: A review ................................................. 81

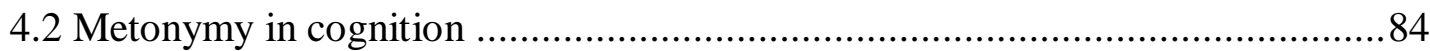

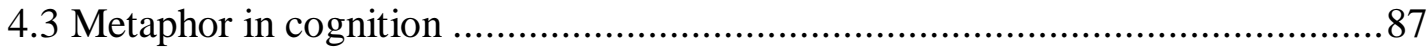

4.4 Metonymy and metaphor in the interactive perspective................................92

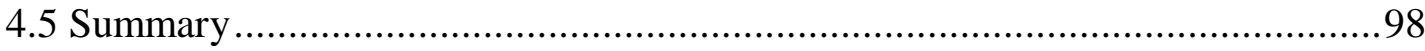

\section{PART TWO METONYMY IN LANGUAGE}

Chapter 5 Metonymy and Grammaticalization ........................................................103

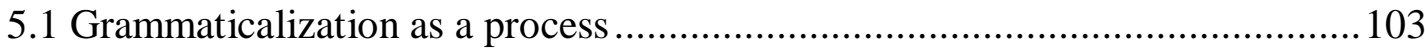

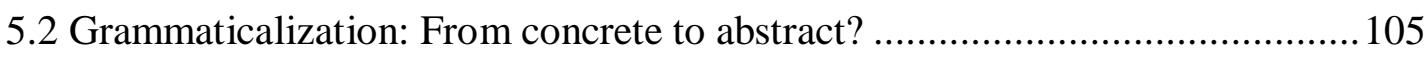

5.3 Metonymy in grammaticalization .......................................................... 111

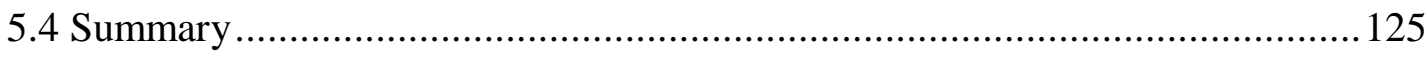


Chapter 6 Metonymy and Meaning Prototype.................................................129

6.1 Meaning in the perspective of cognition........................................................130

6.2 Meaning prototype and cognitive processes ................................................131

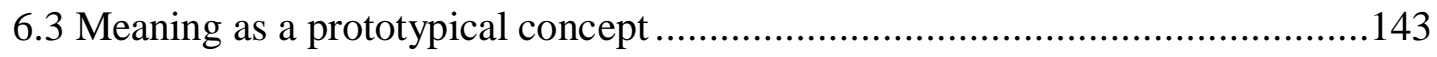

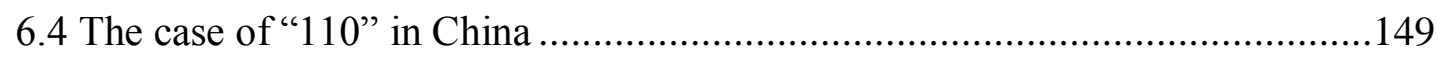

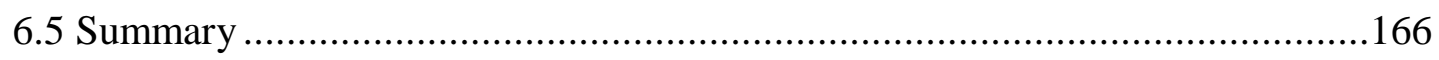

Chapter 7 Metonymy and Football Nicknames ...................................................169

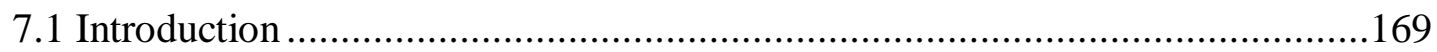

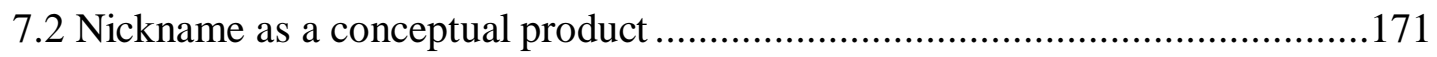

7.3 Football nicknames: Cognitive patterns and mechanisms .............................174

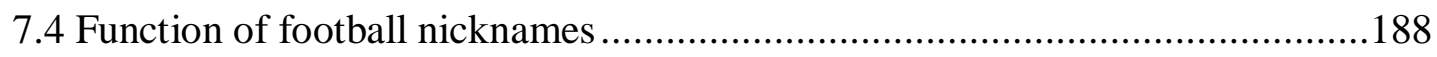

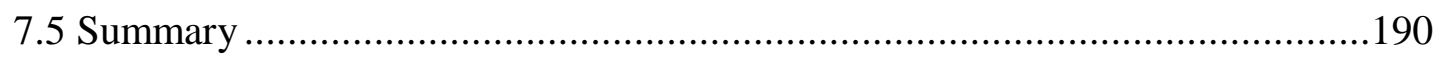

PART THREE METONYMY IN COMMUNICATION

Chapter 8 Metonymy in Dialogic Discourse: Structuring and Developing..........195

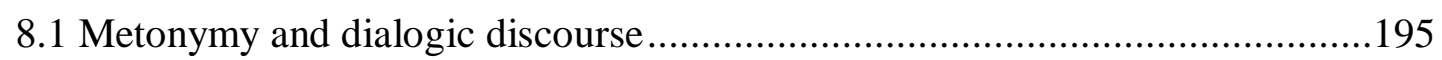

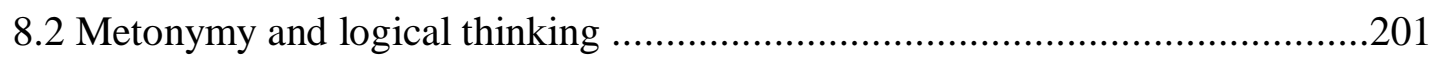

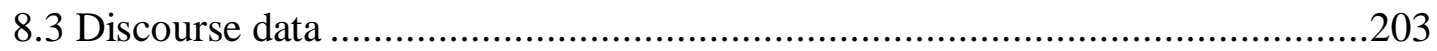

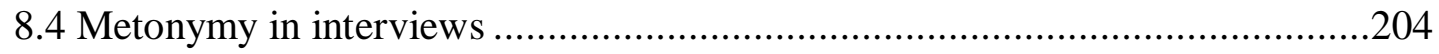

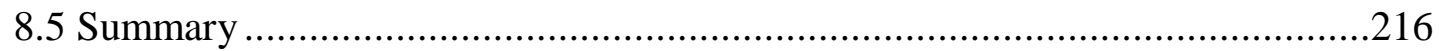

Chapter 9 Metonymy in Dialogic Discourse: Meaning-making, Decisionmaking, and Problem-solving ...............................................................................219

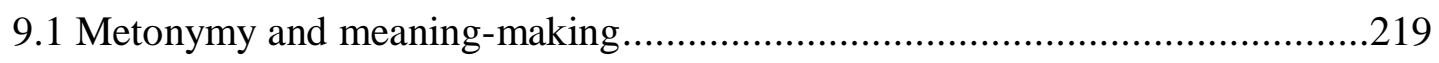

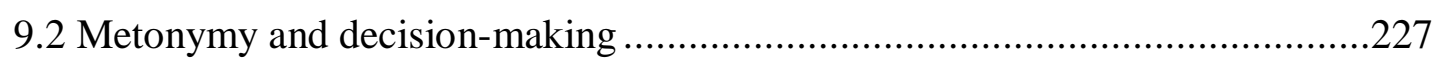

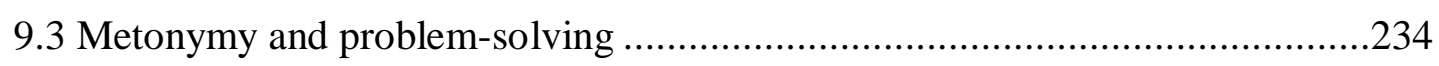

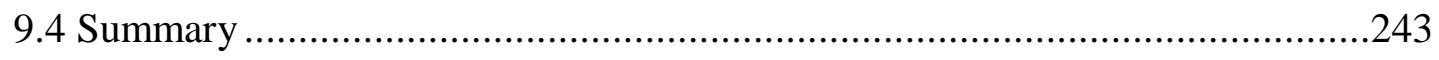

Chapter 10 Conclusion .......................................................................................................245

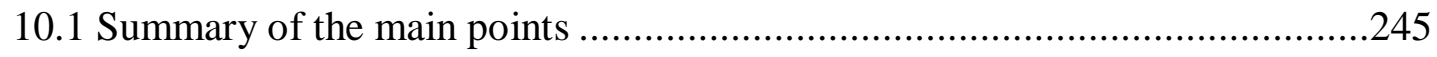

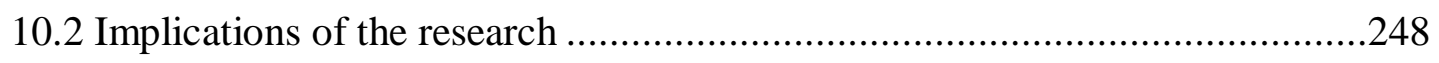

10.3 Areas of future research ....................................................................251

Appendix 1: Examples of the Metaphoric Extension of "110" .................................255

Appendix 2: The Nicknames of Football Club Teams (English Premier League

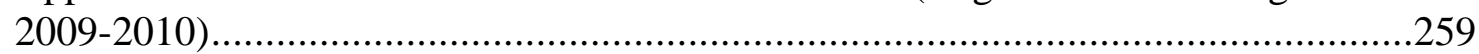

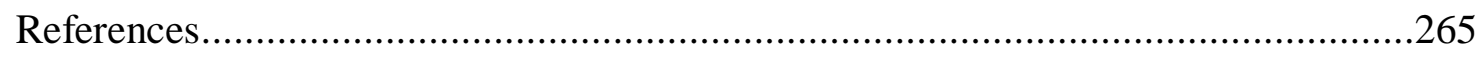




\section{List of tables}

Table 6.1: The numbers and percentage of 110 as different parts of speech...........156

Table 7.1: The patterns in football team nicknames...............................................176

Table 7.2: The cognitive processes underlying football nicknames........................187 


\section{List of figures}

Figure 4.1: The formation of metaphor from the perspective of semiotic cognition.......................................................... 88

Figure 6.1: The semantic representation chart of " 110 " as a noun.........................153

Figure 6.2: The semantic representation chart of " 110 " as an adjective..................155

Figure 8.1: How metonymy structures and develops WSC\#DGI158....................215 


\section{Chapter 1 Introduction}

Metonymy is traditionally considered as a figure of speech in which one word stands for another, related word.

Here are some examples:

- Washington tries to resolve standoff at Camp Ashraf in Iraq. (CNN, May 05, 2011) (Washington for the US government)

- It's always nice to see a friendly familiar face. (The British National Corpus (BNC) ) (face for person)

- The All Whites overcame a shaky start to score a deserved 1-1 draw with China at Wuhan Sports Centre Stadium in Hubei Province on Friday. (The Dominion Post, March 26, 2011) (All Whites for the New Zealand National Football Team, China for The Chinese National Football Team)

- Sir Radford had engaged governesses to teach her, and she wrote a fine hand, played the pianoforte with considerable skill, and had a lovely singing voice. (The Corpus of Contemporary American English (COCA)) (find hand for good handwriting)

- The pen is mightier than the sword. (Edward Bulwer-Lytton) (the pen for literary power, the sword for military force)

- The House was called to order. (the House for the members in the House)

- We have always remained loyal to the crown. (the crown for the queen of UK)

Rhetoricians thus consider metonymy as a pure linguistic phenomenon. But as can be seen further below, Cognitive Linguists view metonymy as more than a linguistic phenomenon. According to Cognitive Linguistics, metonymy is a fundamental cognitive ability where one concept is used as a "reference point" (Langacker, 1999) that provides mental access to another within the same experiential domain.

Metonymy is distinguished from metaphor in Cognitive Linguistics by proposing metonymy as an intra-domain mapping and metaphor as a cross-domain mapping. 
The most typical kind of metonymy is probably the part-for-the-whole type, e.g. face/hand/leg for person in the following examples:

- There is a new face in my office today.

- I need more hands in this work.

- More legs are needed to play the incoming season.

This can be extended to the specific-for-the-general metonymy, where a salient member of an experienced domain or category is used to stand for that domain or category as a whole, e.g. Shakespeare stands for the category of talented writers in "She is a Shakespeare" and cup stands for a filled up drink in "The cup is quite tasty".

The Cognitive Linguistics treatment of metonymy focuses on how the part concept can evoke the whole, how a salient part of an experiential domain can evoke the domain at large, and how a specific member of a category can evoke the whole category and vice versa. Cognitive Linguists, e.g. Kövecses \& Radden (1998), Radden \& Kövecses (1999), Ruiz de Mendoza (2000), and Peirsman \& Geeraerts (2006), use PART to accommodate the specific member, the part concept, and the salient part, and use WHOLE to accommodate the whole concept, the experiential domain, and the whole category. Therefore Cognitive Linguistics focuses on how the PART evokes the WHOLE and vice versa, i.e. PART-WHOLE RELATING.

However, these are descriptions of associations between concepts that are already in place. What Cognitive Linguistics has not investigated in depth is the role metonymy may also play in the formation of those concepts (experiential domains and categories) (i.e. the WHOLE) in the first place. One of the aims of this research is to help fill that niche.

This thesis thus extends the role of metonymy as a cognitive phenomenon even beyond what most Cognitive Linguists have ascribed to it. The term metonymy is used in this research to denote a cognitive mechanism involving PART-WHOLE RELATING process as well as PART-WHOLE FORMING process.

In accordance with Cognitive Linguistics, this research investigates metonymy from a conceptual perspective. Based on current cognitive studies of metonymy, it 
tentatively argues that metonymy is an innate cognitive mechanism involving PART-WHOLE FORMING and PART-WHOLE/PART RELATING processes. I will also illustrate how metonymy is ubiquitous in language and communication, where it serves various discourse functions. This thesis comprises three main parts: METONYMY IN THE MIND, METONYMY IN LANGUAGE, and METONYMY IN COMMUNICATION, and a chapter by way of conclusion. Each part consists of two or three chapters.

This introductory chapter outlines the conception of metonymy as a term in this research (1.1), the reasons for my focus on metonymy (1.2), the theoretical foundations (1.3), the research methodology (1.4), and the content of the three parts (1.5).

\subsection{Metonymy in this research}

Metonymy as a term in this research encompasses such variants as metonymic thinking, metonymic reasoning/inference, metonymic expressions/utterance, and is taken as a prototype of a cognitive mechanism. Since I view metonymy as an innate cognitive mechanism involving PART-WHOLE FORMING and PARTWHOLE/PART RELATING processes, it is thought to underlie and motivate various conceptual activities and language uses; these are all treated under the label of metonymy for the sake of convenience. This research does not make distinctions between metonymy and other expressions containing "metonymy" or "metonymic", and does not distinguish conventionalized metonymy from novel metonymy because they all share the same cognitive mechanism. Once metonymy is taken as a natural cognitive mechanism, it is not necessarily significant to see such notions or classifications as hyponymy and taxonomy as essentially different. Another reason is that this research concentrates on the analysis of the underlying cognitive mechanism whose operations result in such classifications. Therefore, following Kövecses \& Radden (1998) and Radden \& Kövecses (1999), this research also takes PART and WHOLE as prototypes in terms of notion, and does not make distinctions between categorical PART-WHOLE and taxonomical PART-WHOLE for the reason that conceptually there is no difference between them. That may be why Cognitive Linguistics does not distinguish metonymy from synecdoche, and why metonymy 
encompasses a variety of relations as shown in section 2.3 in Chapter 2. In this sense, the prototype WHOLE can sometimes be presented as the general and the prototype PART can accordingly be presented as the specific for the sake of analysis.

When taken as a natural cognitive mechanism, metonymy is more than a reference point phenomenon. Regarding metonymy as a type of reference point phenomenon in the paradigmatic plane ${ }^{1}$, Langacker (1999) actually seems to use metonymy as the reference point phenomenon. To function as a reference point that provides mental access to the target, the source has to have undergone usually co-presence or succession (Blank, 1999) with the target in embodied experience; once the embodied experience is patterned, the appearance of the source (acting as a reference point) can provide mental access to the target. Thus reference point phenomena and other similar activation models in cognition have a prerequisite, i.e. the WHOLE pattern or whatever has already been long established, and this reference point/mental access or activation takes place within the WHOLE pattern. This is only the PARTWHOLE/PART RELATING process. But metonymy in this research consists of PART-WHOLE FORMING process as well as PART-WHOLE/PART RELATING process. That is, although it is suggested that human beings are born with a WHOLE (body schema) which influences their thinking, it is only a prototype which needs to be formed or configured in embodied experience. The configuration of the WHOLE is based on specific aspect(s) of what is perceived and conceived, and this is achieved through PART-WHOLE FORMING process, in which a WHOLE pattern are mentally formed. This WHOLE pattern might be very rough initially but would take shape and become enriched in the development of embodied experience.

\subsection{Focus on metonymy}

Metonymy is traditionally viewed as a figure of speech using the name of one thing for something else associated with it, the main types being relationships of causeeffect, part-whole, whole-part, and producer-product. Following structural linguistics'

\footnotetext{
${ }^{1}$ In Langacker 's view (1999, chapter 6 Reference point constructions), there are many types of reference point phenomena, which occur in both syntagmatic plane (i.e. syntagmatic relationships among sentential elements, e.g. anaphora, non-possessive constructions, quasi-possessive constructions) and paradigmatic plane; metonymy is the primary one of those reference point phenomena in the paradigmatic plane, i.e. metonymy occurs when an expression that normally designates one entity is used instead to designate another, associated entity.
} 
view of metonymy as a figure of sense based on a contiguity relationship (Jakobson, 1971/2002; Ullmann, 1962), lexical semantics regards metonymy as a lexical meaning extension mechanism (e.g. Goossens, 2000; Nerlich, 2010); Cognitive Linguistics takes up metonymy as a figure of thought and a cognitive process based on conceptual contiguity (e.g. Dirven, 2002; Gibbs, 1994). Metonymy in Cognitive Linguistics is considered to be a conceptual phenomenon which structures and shapes human thinking and language (Lakoff, 1987b; Lakoff \& Johnson, 1980; Lakoff \& Turner, 1989; Langacker, 1987). It is also considered to be ubiquitous in language and to have cognitive and communicative functions (Barcelona, 2002b; Langacker, 1999; Radden, 2005).

Metonymy has undergone an upsurge in research since Lakoff \& Johnson's seminal book Metaphors We Live By, but metonymy research still lags behind metaphor research both in quantity and in area of application. Furthermore, even in the cognitive research on metonymy, there are still aspects in need of further clarification and study, e.g. the nature of metonymy, metonymy in thought, metonymy in language and discourse, and the application of metonymy in other fields. Apart from that, the cognitive research on metonymy has not yet formed a uniform theory of metonymy, e.g. the Reference Point/Mental Access view, the Conceptual Blending view, the Contingent Indexical view, and the Cognitive Domain view. These approaches to metonymy are attempts to clarify metonymy as a cognitive process in language and thought, endeavouring to interpret the conceptual contiguity in a manifest manner.

The Reference Point/Mental Access view regards metonymy as a reference point phenomenon in which the source, acting as the reference point, provides mental access to the target in the same ICM. It seems to be a very broad view of cognitive construal. According to this view, metonymy is only a common type of reference point selection that operates in the paradigmatic plane, i.e. "an expression that normally designates one entity is used instead to designate another, associated entity", and other reference point phenomena operate in the syntagmatic plane ${ }^{2}$ (Langacker, 1999, pp. 198-202, 207-208). Langacker (1999) in fact does not always distinguish clearly between metonymy and reference point (see e.g. Barcelona (2011,

\footnotetext{
${ }^{2}$ See the previous footnote.
} 
p. 30) and Langacker (2009)). The question of how mental access can take place or be triggered seems to be left unanswered.

The Conceptual Blending view takes metonymy as a phenomenon involving blending the mental spaces of the source and the target. Like the Reference Point/Mental Access view, the blending view is broad enough to possibly encompass other mental activities as well, e.g. metaphor. It seems that these two views have not yet, in theory, been applied to the distinction between metonymy and metaphor.

The Contingent Indexical view takes metonymy as an indexical relation between the source and the target, and the indexical relation is cancellable. This view seems to focus merely on temporary or novel metonymic phenomena in language use, excluding those conventionalized metonymic expressions and those phenomena in which metonymy is based on iconic relation (see e.g. Warren, 1998, 2002).

The Cognitive Domain view employs "domain" to feature metonymy as an intradomain mapping. Owing to the vagueness of terms such as domain and mapping and the application of the terms in both metonymy and metaphor, it is difficult to have a clear-cut distinction between these two cognitive processes, which are assumed to be quite different from each other.

Despite these different views, there seems to be a consensus in the cognitive perspective of metonymy that metonymy is a cognitive process underlying various linguistic levels such as phonological, morphological, lexical, syntactic, discoursal levels (see e.g. AI-Sharafi, 2004; Barcelona, 2000b, 2002b, 2004, 2005a, 2007a, 2007b; Brdar, 2007; Dirven \& Pörings, 2002; Langacker, 2004; Panther et al, 2009; Radden, 2005; Ruiz de Mendoza \& Otal Campo, 2002; Ruiz de Mendoza \& Pérez Hernández, 2001), underlying speech acts and pragmatic inferences (Barcelona, 2003a, 2005b; Panther \& Radden, 1999; Panther \& Thornburg, 2003b; Thornburg \& Panther, 1997), and other cognitive and communicative activities (see e.g. Ruiz de Mendoza, 1997, 1998; Taylor, 2002a). Metonymy is hence claimed to be elementary and more central and basic than metaphor in language and cognition (Barcelona, 2000c, 2002b, 2005a; Gibbs, 1994; Kalisz, 2007; Mittelberg \& Waugh, 2008; Mittelberg \& Waugh, 2009; Radden, 2005); and to be the necessary base for most 
metaphors (Barcelona, 2000a, 2000b; Dirven \& Pörings, 2002; Panther \& Radden, 1999).

While much work has been done on metonymy in Cognitive Linguistics, most of the research, as mentioned above, seems to be confined to language in general, and the metonymic motivation of and constraints on linguistic phenomena in particular, e.g. lexical semantics (words, phrases, idioms), syntactic structure, speech acts, and interaction between metonymy and metaphor; and mostly, confined to intuitive data (Gibbs, 2006); very little research has been done on discourse. Indeed, the dynamic aspect of metonymy in dialogic discourse is rarely touched on, e.g. how metonymy from the speaker influences the listener and thus what response is invited from, or what psychological change occurs in the listener.

In addition, even in the most studied area of metonymy, i.e. metonymy in language, there are also some fields left unploughed or not well developed, e.g. metonymy in grammaticalization, metonymy in meaning prototype. Another question concerns the cognitive nature of metonymy. If metonymy is a cognitive process/model, as claimed in Cognitive Linguistics, is this cognitive process/model self-driven or motivated by other cognitive elements? That is, is metonymy a mere cognitive process or rather a natural cognitive mechanism? ${ }^{3}$ So far, it seems this kind of question has not been answered, or even raised.

Moreover, the claim that metonymy is a cognitive process seems to be based merely on the conceptual analysis of language phenomena and needs to be empirically evidenced in cognitive science and neuroscience. In this case it can be taken as ubiquitous in language-involved activities. But, is it only ubiquitous in language and language-involved cognitive activities or is it also significantly operative in cognitive activities beyond language or where the effect of language is least felt? Since these questions resonate beyond language phenomena, it might be necessary to look at the

\footnotetext{
3 "Process" and "mechanism" are used here not as opposing notions but in the sense that processes are operational functions of a mechanism. Here "mechanism" is stressed as a comprehensive system and "process" is the actual operation of a mechanism. This means that a process cannot tell the whole story but a mechanism does. Metonymy in this research is seen as innate and needs no other force for its operation and function; it is not developed or learned or trained; it is an independent system that has its own processes such as PART-WHOLE FORMING, PART-WHOLE/PART RELATING. It is from this stance that the distinction is made between "mechanism" and "process".
} 
field of cognitive science and neuroscience for the answers. Otherwise, the claim of metonymy as a cognitive process based on language phenomena only would not be as convincing and robust.

Studying or viewing language from the cognitive perspective has to be based on and consistent with recent research results of other disciplines in cognitive science (cf. Evans \& Green, 2006; Gibbs, 2006; Lakoff, 1987b; Lakoff \& Johnson, 1999). Language, though a vital part, is only part of cognition. Thus metonymy, treated as a cognitive process, has to be evidenced in cognition without language or with language as the least felt factor; and more importantly, the cognitive driver of this process has to be evidenced beyond language influence, if taken as a cognitive process/model.

\subsection{Theoretical foundations}

This research is based on the theoretical principles of Cognitive Linguistics ${ }^{4}$ and Embodied Cognition Theory. Embodied Cognition suggests that the body plays a crucial role in cognition and cognition is eventually grounded on the perceptual experience in the physical and socio-cultural environment. Following Embodied Cognition Theory, Cognitive Linguistics advocates embodied meanings and a distinction collapse between semantics and pragmatics. This is significant in theory to the conceptual analysis of language and language use, and it indicates that embodied meaning is encyclopaedic in nature. The following sections are devoted to a brief explanation of the main ideas so that the theoretical orientation of Cognitive Linguistics and Embodied Cognition Theory can become clear.

\subsubsection{The fundamentals of Cognitive Linguistics}

Cognitive Linguistics is "the study of language in its cognitive function, where cognitive refers to the crucial role of intermediated informational structures in our encounters with the world" (Geeraerts \& Cuyckens, 2007a, p. 5). It studies the relation between human language, the human mind, and human socio-physical

\footnotetext{
${ }^{4}$ Following Geeraerts \& Cuyckens (2007a) and others, the term Cognitive Linguistics (CL) is employed here in order to be distinguished from the Chomskyan cognitive approach to language, which views language as an innate independent faculty. For details on the difference between these two approaches, see Geeraerts (2006a), Evans \& Green (2006), Taylor (2002b), also see Janda (2006), Lee (2001), Ungerer \& Schmid (2006), Gonza'lvez-Garcı'a (2009).
} 
experience (Evans, et al, 2007a), which is destined to be interdisciplinary by nature: It involves

Psychology (both Cognitive and Social), Anthropology (both Evolutionary and Cultural), Sociology, Philosophy (especially via the study of categorization), and indeed, any field in which scholars study conventionalized knowledge systems (conceptual systems) deployed in human interaction. ${ }^{5}$

This cognitive approach to language focuses on "language as an instrument for organizing, processing, and conveying information", and addresses such topics as "the structural characteristics of natural language categorization, the functional principles of linguistic organization, the conceptual interface between syntax and semantics, the experiential and pragmatic background of language in use, and the relationship between language and thought" (Geeraerts, 1997, p. 7). To put it simply, Cognitive Linguistics looks at language system and language use through a conceptual lens and examines how language functions at the interface of brain, mind, and communication.

It has been made clear in Cognitive Linguistics that this approach to the study of language is not a single articulated theory of language, but a flexible framework or enterprise with some common core ideas and principles (Vyvyan Evans et al., 2007a; Geeraerts, 1997, 2006a; Geeraerts \& Cuyckens, 2007a; Janda, 2006; Radden, 1992; 2008). The ICLA lists the common basis as

The idea that language is an integral part of cognition that reflects the interaction of cultural, psychological, communicative, and functional considerations; that language can only be understood in the context of a realistic view of conceptualization and cognitive processing; and that any theoretical conception of language must be compatible with what is known about neurological organization and function.

This is the basis shared by different approaches under the label of Cognitive Linguistics. Different scholars stress different aspects in their writing on Cognitive Linguistics. The fundamental hypotheses of Cognitive Linguistics, as derived from

\footnotetext{
${ }^{5}$ See International Cognitive Linguistics Association (ICLA) website http://www.cognitivelinguistics.org/about.shtml, retrieved on 15 December 2009
} 
representative books and papers on Cognitive Linguistics, are set out in following paragraphs.

Croft \& Cruse (2004, p. 1) see three major guiding principles in the Cognitive Linguistic approach to language:

1) Language is not an autonomous cognitive faculty;

2) Grammar is conceptualization;

3) Knowledge of language emerges from language use.

In Croft \& Cruse (2004, pp. 1-4), the representation and the process of linguistic knowledge (knowledge of meaning and form) are no different from the representation and the process of other conceptual structures; linguistic knowledge is essentially conceptual structure, which is subject to construal; knowledge of language originates from the cognition of specific utterances on specific occasions of use.

Geeraerts (1997, p. 8) and Geeraerts \& Cuyckens (2007a, p. 5) present three fundamental characteristics of Cognitive Linguistics:

1) The primacy of semantics in linguistic analysis,

2) The encyclopaedic nature of linguistic meaning,

3) The perspectival nature of linguistic meaning.

In Geeraerts (1997) and Geeraerts \& Cuyckens (2007a), meaning is placed at centre stage and further described from the conceptual perspective. The first point emphasises the crux of meaning in Cognitive Linguistics, the second point defines the encyclopaedic nature of meaning in language, and the third further underscores in language the mediating role of the mind in its encounter with the objective world, i.e. in organizing knowledge, language embeds the perspectives and experiences of the world.

In line with this, Geeraerts (2006a, pp. 3-6) emphasises the point that the most basic characteristic is that "language is all about meaning", which can be elucidated as the following four specific characteristics of Cognitive Linguistics:

1) Linguistic meaning is perspectival; 
2) Linguistic meaning is dynamic and flexible;

3) Linguistic meaning is encyclopaedic and non-autonomous;

4) Linguistic meaning is based on usage and experience.

In Geeraerts (2006a), linguistic meaning is further developed. According to him, language reflects the view of the world, it also shapes the perspective of view ${ }^{6}$; the world is changing, different people have different experiences of the world and may have different perspectives even on the same entity in the world, which brings in different interpretations and thus dynamic and flexible meaning. In this sense, meaning is constructed through language by the mind, which involves personal experience of different scenarios.

Evans \& Green (2006) and Evans et al (2007a), following Lakoff's (1990) generalization commitment and cognitive commitment ${ }^{7}$, put forward four guiding principles:

1) Conceptual structure is embodied;

2) Semantic structure is conceptual structure;

3) Meaning representation is encyclopaedic;

4) Meaning construction is conceptualization.

These principles accentuate the embodied nature of cognition and the conceptual nature of meaning. They show that conceptual structure results from the embodied experience and the structure of language reflects the conceptual structure in mind ${ }^{8}$. They also indicate that encyclopaedic meaning originates from actual use and is thus dynamic.

\footnotetext{
${ }^{6}$ Language as a product reflects others' perspectives of viewing the world. At the same time, it influences our perspective of view. That is, language, while reflecting the embodied experience of the interaction with the world, would inevitably shape how one looks at the world once one thinks in the language. This is also a point of linguistic relativity, see e.g. Gumperz \& Levinson (1996).

${ }^{7}$ In Lakoff (1990), Cognitive Linguistics is seen as defined by the commitment to linguistic generalization that is in consistent with empirical discoveries about the nature of the mind/brain.

${ }^{8}$ When it is said that the structure of language or language reflects the conceptual structure in mind, it is from the point of view of language as a system in use, i.e. as a product, language reflects the conceptual structure. However, when this product as a system is to be acquired or learned, it shapes the way the acquirers or learners (especially children) think. That is to say, language is a product of embodied human cognition and meanwhile it exerts influence on the language acquirer/learner/user in terms of thinking. It is in this sense that Boroditsky and her colleagues contend that language shapes the way one thinks.
} 
Evans (2010a) generalizes from the cognitive literature that Cognitive Linguistics takes the following assumptions as its guide:

1) Language is the outcome of general properties of cognition;

2) Conceptual representation is the outcome of the nature of the bodies humans have and how they interact with the socio-physical world;

3) Grammar is conceptual in nature;

4) Meaning, as it emerges from language use, is a function of the activation of conceptual knowledge structures as guided by context.

Evans stresses that language is part of the human cognitive faculty and it is conceptual in nature; conceptual structure is embodied and originates from human experience of the world; and meaning comes from the activation of the conceptual knowledge by the context.

From the above and other works on Cognitive Linguistics, e.g. Evans et al (2007b) Fauconnier (2002), Geeraerts (2006b), Geeraerts \& Cuyckens (2007b), Janda (2006), Kemmer \& Barlow (2000b), Kövecses (2005), Kristiansen et al (2006), Lee (2001), Radden \& Dirven (2007), Rudzka-ostyn (1988), and Taylor (2002b), it could be claimed that Cognitive Linguistics is theoretically:

1) "Embodied cognition/experience" grounded,

2) "Conceptual" oriented,

3) "Meaning" focused,

4) "Usage" based,

5) "Data" driven.

In Cognitive Linguistics, human cognition and experience are determined by the human body; the structure and the features of the human body determine what can be cognized and experienced and how. Experiments show that right-handers and lefthanders perform actions differently and they use correspondingly different areas of the brain for representing action verb meanings (Willems et al, 2010). Right-handers tend to associate rightward space with positive ideas and leftward space with negative ideas, but left-handers associate rightward space with negative ideas and leftward with positive ideas (Casasanto, 2009). 
Cognitive Linguistics sees language as an integral part of human cognition and assumes that language study can be enlightened by what is known about the mind, and aims to provide a cognitively feasible account of language phenomena (Langacker, 1987; Taylor, 2002b). Language is thus viewed from the conceptual perspective, and is taken as a very special component of overall human cognitive capacities; it is regarded as a track to thinking and to be able to reflect the conceptual structure of the mind.

Cognitive Linguistics supposes that the speaker's linguistic system is fundamentally grounded in usage events, i.e. in the speaker's producing and understanding language, and linguistic representations are emergent entities (Kemmer \& Barlow, 2000a); knowledge of language emerges from language use (Croft \& Cruse, 2004), study of language is study of language use (Fauconnier, 2002). It underscores the importance of actual language use as the source of language knowledge and language use, and stresses the intimate relation between language knowledge and language use. Knowledge of language and knowledge of language use are both encyclopaedic knowledge, which is structured and emerges in context and thus dynamic in nature. Hence meaning is not stored in entities and does not reside in linguistic units, but rather is constructed on-line in the mind of the language user as discourse unfolds in context (Fauconnier, 2002). Language does not represent meaning, and linguistic representation is just a prompt for the construction of meaning at the conceptual level in a particular context (Evans, et al., 2007a; Fauconnier, 2002; Radden, et al., 2007a). Meaning construction involves both the hearer and the speaker in making inferences (Radden, et al., 2007a); it is a mental blending of encyclopaedic knowledge and the actual situation. In this sense, the distinction between semantics and pragmatics breaks down (Vyvyan Evans, 2010a; Fauconnier, 2002). The meaning of language is therefore viewed as a conceptual configuration which comes from embodied experience. Embodied experience indicates that language is for use and from use, the meaning of language originates from actual language use and develops in actual use, it is based on usage. The study of language thus becomes the study of the way of expressing ideas and thoughts (Dirven \& Verspoor, 1998), and should be based on the analysis of language data. The methodology of top-down and bottom-up in linguistic research is expected to be integrated accordingly. 
These fundamentals of Cognitive Linguistics also suggest that encyclopaedic knowledge plays a crucial role in both language and thought, since meaning is encyclopaedic and conceptual in nature. Encyclopaedic knowledge is well organized in the mind and may be considered as a WHOLE prototype consisting of independent but interacting networks, which might be individually taken as a WHOLE when they function primarily on their own independently. This comparatively independent network of knowledge involves groups of factors, which might be individually taken as a PART when their operations are primarily legitimated within this network. Thus understood, the use of WHOLE and PART in this research follows Kövecses \& Radden (1998) and Radden \& Kövecses (1999) in their interpretation of WHOLE and PART. Since encyclopaedic knowledge is assumed to be structured in the form of Idealized Cognitive Models or ICMs (Lakoff, 1987b, p. 68), the WHOLE is similar to ICM / Frame ${ }^{9}$ (Barcelona, 2011) in this sense.

\subsubsection{The essentials of Embodied Cognition Theory}

Metonymy research is done within the framework of Cognitive Linguistics, Cognitive Linguistics as a discipline is theoretically grounded on phenomenology, embodied realism, experientialism, or rather, on what is commonly termed the theory of Embodied Cognition (Johnson, 1987, 2007; Johnson \& Lakoff, 2002; Lakoff, 1987b; Lakoff \& Johnson, 1980; Lakoff \& Turner, 1989; Langacker, 1987; Rohrer, 2007). Because this research is related to Embodied Cognition in regard to attempting to take metonymy as a natural cognitive mechanism since birth, it will only set forth the guiding principles of Embodied Cognition Theory.

Embodied Cognition stresses the role of the body in cognitive activities and cognitive development and claims that cognition is shaped or determined by the body. Embodied Cognition grounds concepts or conceptual processing on perceptual and sensorimotor activities, and eventually, on neural patterns in the brain; it also claims that cognition is multimodal (Barsalou, 1999b), and that cognition is

\footnotetext{
${ }^{9}$ Frame, in this sense, was first used by Fillmore (2006(1972)). It is used here and elsewhere in Cognitive Linguistics as a form of structuring knowledge as similar to ICM, i.e. they are rich models of a relatively specific area of experience, whereas Domains are general classificatory schemes of experiential areas. Thus a Frame will normally recruit elements from many taxonomically different domains: the UNIVERSITY frame includes students, staff, faculty, lecture theatres, library, buildings, finance, etc.
} 
constructed, and develops in bodily interactive experience with the physical and socio-cultural environment; it takes cognition as goal-directed; see e.g. Anderson (2003, 2007, 2008), Cowart (2009), Gallagher (2005), Gibbs (2006), Johnson (1987, 2007), Lakoff \& Johnson (1999), Marsen (2009), Pecher \& Zwaan (2005), Rohrer (2007), Thelen (2000), Varela et al. (1991), Ziemke (2003), and Ziemke et al. (2007).

In Embodied Cognition, reality is constructed by the nature of unique human embodiment, and the construal of reality is likely to be mediated by the nature of bodies (Evans \& Green, 2006). According to Cowart (2009), Embodied Cognition has the following as its key characteristics:

1) Primacy of goal-directed actions occurring in real-time;

2) Form of embodiment constrains kinds of cognitive processes;

3) Cognition is constructive.

It is contended that thought results from the ability to act in the environment and human cognitive actions are goal-directed in certain settings, which suggests that in cognitive actions attention is only paid to what is meaningful to the observer in that situation. Thus it is possible to look without seeing or "inattentional blindness" (Mandler, 2004; also see O'Regan, Rensink, \& Clark, 1999). It is in this sense that Cognitive Linguistics claims that linguistic meaning is perspectival. Goal-directed actions suggest that meaning is significant and it derives from cognitive actions; actions in real-time shows the significance of contextual information. Thus the first feature of Embodied Cognition focuses on meaning and usage events, and is related to the Cognitive Linguistic advocacy of meaning in actual use: Cognitive Linguistics takes meaning as the central stage and as emerging from usage.

The second feature indicates that the particular ways of embodiment influence how goal-directed actions are performed in the world and the particular sensorimotor experience connected with these actions serves as the basis for categorization and conceptualization and thus shapes their cognitive functions. This feature does not deny the significance of environmental factors, which can influence not only what options are available, but also why one option is preferred over another when performing a particular goal-directed activity, for these factors in the environment have been taken into account by the sensorimotor experience in the performance of 
the action. Thus this characteristic hosts the subject and the context harmoniously and treats the interaction between them more comprehensively.

The third feature follows the first two. Since cognition is goal-directed in a certain environment and conceptualization and categorization are based on sensorimotor experience, it is unavoidable that Embodied Cognition is constructive: It depends on many relevant factors, such as the goals, actions, the form of embodiment, and the functioning of sensorimotor modalities. Humans are initiating and active in constructing sensorimotor representations based on those environmental features that are directly relevant to the goal-directed action they are performing. This feature is also relevant to the view of meaning and encyclopaedic knowledge in Cognitive Linguistics, e.g. "Meaning, as it emerges from language use, is a function of the activation of conceptual knowledge structures as guided by context (Evans, 2010a)".

Taken in the aggregate, Embodied Cognition is a comprehensive theory of both language and cognition. It places more emphasis on the subjective aspect (e.g. goals), the objective aspect (e.g. the real time environment, the embodied form), and the interaction of the subjective and the objective (e.g. meaning construction and contextual constraints). It advocates an integrated analysis of any cognitive activity, taking into account every functional component involved in the activity and only placing the weight on the aspects most concerned. In the light of Embodied Cognition, cognitive activities, such as language communication, need to be considered as a whole event scenario and analysed in accordance with different goals and perspectives. Both the Embodied Cognition Theory and the fundamentals of Cognitive Linguistics provide theoretical guidelines for language analysis, especially the conceptual approach to the ways that language is used, deliberately or unconsciously.

Embodied Cognition values the significance and function of the body in cognition, which operates even before birth:

Prenatal bodily movement has already been organized along the lines of our own human shape, in proprioceptive and cross-modal registrations, in ways that provide a capacity for experiencing a basic distinction between our own embodied existence and everything else (Gallagher, 2005, p. 1). 
Thus human beings were born with a body schema ${ }^{10}$ that impacts the bodily experience and cognition. In this sense, "experience is complex and holistic from its very beginning" (Gallagher, 2005, p. 5). Starting from this point, the body schema may be taken as a prototype WHOLE, which shapes the mind without conscious awareness. This might be considered as the main reason for the tentative claim of metonymy as a natural cognitive mechanism in this research.

\subsection{Methodology}

This research is predominantly corpus-based in the analysis of language and dialogic discourse. PART ONE is based on experiments in cognitive science and combines both qualitative and quantitative analyses.

For the theoretical analysis, i.e. for the tentative argument of metonymy as a natural cognitive mechanism of attentively selecting a prominent part in an experience pattern for the whole or vice versa, the empirical evidence is taken from the experimental results in cognitive science and neuroscience, within the framework of Embodied Cognition. Some experiments are presented in brief and the results are interpreted relevantly.

In PART TWO, the illustrations and evidence are mainly case studies from data gained by corpus and statistical approaches, combining both qualitative and quantitative strategies. For example, " 110 " as the case study of a metonymymotivated dynamic meaning prototype is from the internet-based corpus Sketch Engine Corpus, from Internet-ZH, to be specific, and the (Chinese) People Daily ${ }^{11}$. Internet-ZH is part of the Sketch Engine Corpus established in 2005 by Serge Sharoff, who thinks that internet-based corpora from search engines are better than

\footnotetext{
${ }^{10}$ A body schema, according to Gallagher (2005, p. 25), is a system of sensory-motor capacities that function without awareness or the necessity of perceptual monitoring. It involves certain motor capacities, abilities, and habits that both enable and constrain movement and the maintenance of posture. It continues to operate, and in many cases operates best, when the intentional object of perception is something other than one's own body. In Gallagher's view, embodiment functions in the form of body image and body schema, which are interrelated in cognition, though each has its own responsibilities, e.g. a body image involves more than occurrent perceptions, and it can include mental representations, beliefs, and attitudes where the object of such intentional states (that object or matter of fact towards which they are directed, or that which they are about) is or concerns one's own body. In this research, a WHOLE prototype is employed to cover both the notions of body image and body schema due to their close interrelation and the nature of my research (it is not specifically a neurological research on body schema or body image).

${ }^{11}$ More details on the corpora are given in Chapter 6.
} 
balanced/ representative corpus such as BNC (British National Corpus) in terms of authorship, mode, audience, aim, and domain. Football nicknames as the case of metonymically-motivated daily language phenomenon are done in an organized corpus which originates from the (English) Barclays Premier League. Grammaticalization is discussed and analysed in a qualitative examination.

The METONYMY IN COMMUNICATION part is also based on corpus data. Most of the dialogic discourse examples are selected from corpora. The first is Corpus WSC (Wellington Corpus of Spoken New Zealand English) established by School of Linguistics and Applied Language Studies, Victoria University of Wellington, under the leadership of Janet Holmes, Bernadette Vine and Gary Johnson in 1998, which contains many recorded public and private dialogues ${ }^{12}$; The second is the ICE (International Corpus of English) (New Zealand, Singapore) ${ }^{13}$, each ICE corpus consisting of one million words of spoken and written English produced after 1989 while following a common corpus design; only the dialogic part of these corpora are employed.

\subsection{The main points of the three parts}

PART ONE of this thesis reconsiders metonymy as a cognitive process as advocated in current Cognitive Linguistics, and it tentatively argues that metonymy is instead an innate cognitive mechanism unconsciously operating in such basic cognitive activities as perceptual and conceptual categorizations, concept formation and development, memory, and inductive inference beyond language influence. This innate cognitive mechanism involves two cognitive processes: PART-WHOLE FORMING and PART-WHOLE/PART RELATING; the first is responsible for the establishment of an experienced pattern or formulation, and the second is responsible for relating PART to PART and relating the PART to the WHOLE pattern and vice versa. The latter is what is focused on in the Cognitive Linguistic literature of metonymy. PART ONE of this thesis also analyses the interaction between metonymy and metaphor in cognition without language, arguing metaphor is logically and conceptually grounded on metonymy, thus developing and validating

\footnotetext{
${ }^{12} \mathrm{http} / / /$ www.victoria.ac.nz/lals/research/corpora/wcs.aspx

${ }^{13}$ More details on ICE-NZ are given in Chapter 8.
} 
the Cognitive Linguistic claim from experimental research in cognitive science that metonymy is the operative base of metaphor.

Cognitive Linguistics views language as part of human cognitive abilities and (1) claims that language reflects conceptual structures, i.e. language is cognitively motivated (cf. Cuyckens et al, 2003; Evans et al, 2007a; Vyvyan Evans \& Green, 2006; Geeraerts, 2006a; Geeraerts \& Cuyckens, 2007a; Janda, 2006; Radden \& Panther, 2004), and (2) advocates Embodied Cognition as the theoretical framework (see e.g. Vyvyan Evans \& Green, 2006; Fauconnier, 2002; Geeraerts \& Cuyckens, 2007b; Johnson, 1987, 2007; Lakoff, 1987b; Lakoff \& Johnson, 1999; Ziemke, 2003). Embodied Cognition accounts stress the roles of the body and the interactive experience between the body and the physical and socio-cultural environment in cognitive activities, claiming that cognition is virtually grounded in the sensorimotor activities in the interactive experience, i.e. perception and action (see e.g. Anderson, 2003; Cowart, 2009; Frank et al, 2008; Gallagher, 2005; Gallese \& Lakoff, 2005; Gibbs, 2006; Glenberg et al, 2005; Pecher \& Zwaan, 2005; Rohrer, 2007; Ziemke et al, 2007), and eventually on neural action and networking (Barsalou, 2008b; 2008c; Barsalou \& Wiemer-Hastings, 2005; Berlucchi \& Aglioti, 1997; Casasanto, 2009; Chiel \& Beer, 1997; Kosslyn et al, 2001; Lakoff, 2009; Mashal et al, 2007; Willems \& Hagoort, 2007). Reviewing and examining some basic cognitive activities within the Embodied Cognition framework from research experiments in developmental psychology, cognitive psychology, neuroscience (e.g. how categories are formed, how a concept is formed and develops, how memory works in a subject's prelinguistic period and in subjects with damaged language ability or memory) suggests that metonymy definitely operates and endures, even when the metaphoric process is lost. This finding and the question whether metonymy is a cognitive process or a natural mechanism, in the aggregate, leads to the view that metonymy is actually a natural cognitive mechanism rather than merely a cognitive process that needs a driver.

The intensive cognitive research on metonymy and other approaches to metonymy deepen the understanding of metonymy. Although the "contiguity" relationship in metonymy is variously interpreted as "non-similarity" or "intra-domain mapping" or 
"contingent indexical" or "mental reference point"14, it is generally viewed as a relation between PART and WHOLE (cf. Radden \& Kövecses, 1999; Ruiz de Mendoza, 2000). With the PART-WHOLE relation as a prototypical category, metonymy is classified in terms of the types of contiguity involved in three dimensions, e.g. from PART-WHOLE physical contact to adjacency without contact, from bounded PART-WHOLE to unbounded, from spatial to temporal, spatiotemporal to categorical domain (Peirsman \& Geeraerts, 2006). This prototype account of metonymy is based on the prototype interpretation of "contiguity" (cf. Barcelona, 2011), whereas other scholars (Barcelona, 2002a, 2003b, 2011), argue that prototypical metonymy is only part of the schematic metonymies, which include Purely Schematic, Typical, Simply Typical and Prototypical metonymies according to the degrees in metonymicity. Both of these accounts require the PART-WHOLE relation to take place within the WHOLE (i.e. the same ICM/Frame). In fact, this PART-WHOLE relating does not only operate in cognition with language, it also underpins perceptual categorization and conceptual categorization before language is developed and acquired, and in inductive reasoning before language takes effect. It also operates in the cognitive activities of those subjects whose language ability is somehow damaged. Metonymy is thus evidenced to be a natural cognitive mechanism, which is inherent and develops together with the organism. Cognition is decided by the body in general (e.g. the body schema), and by the body structure in particular. The body structure determines that not everything can be seen at once; even in what is seen, only part of it is focused and only something concerned in the situation is attended to at the time. Whether it is perceptual categorization or conceptual categorization, attention is needed in this cognitive activity. Thus this cognitive mechanism of metonymy makes it possible to pick up PART of what is perceived and to take it as the WHOLE. However, this PART-WHOLE relating is warranted by an experience pattern, which is formed in categorizing when attentive perceiving takes place. In this sense, metonymy operates in the mind even without language.

\footnotetext{
${ }^{14}$ Strictly speaking, only the first two are interpretations of the "contiguity" relationship, because they explain what counts as contiguity. On the contrary, the last two just explain the working mechanism, (i.e. how metonymy works), e.g. the source provides indexical reference to the target; the source, acting as a reference point, provides mental access to the target. Thus the last two do not interpret what counts as a contiguity relation.
} 
PART TWO of the thesis focuses on metonymy in language, providing further evidence on metonymy as a natural cognitive mechanism. Recent decades have seen a surge of research on metonymy in language, therefore, emphasis will be given to those areas that are ignored or rarely covered in the literature. The thesis takes up those disregarded aspects of metonymy as a motivation in language and explores the crucial roles of metonymy in the process of grammaticalization, in dynamic meaning prototypes, and football nicknames. Grammaticalization has been widely viewed for a long time as a syntactic feature with little relevance to semantic or meaning aspect. By contrast, through analysis of both English and Chinese cases, it is argued here that grammaticalization is essentially a conceptual development phenomenon and is largely motivated by metonymy. The notion of the prototype is in fact well employed in lexical semantics and cognitive semantics. However, the dynamic nature of prototyping is not fully understood and realized. The second chapter of PART TWO argues, through reviewing views on lexical prototype and clarifying how meaning prototype develop in a Chinese case analysis, that metonymy motivates meaning prototype and determines its dynamic nature in most cases. It suggests that metonymy underlies the process of grammaticalization and the development of meaning prototype. By the same token, the last chapter of this PART also takes a case study to demonstrate that metonymy functions in daily language experience without conscious awareness, e.g. in football nicknames, and underlies daily language use. PART ONE mainly focuses on metonymy as an inherent cognitive mechanism operating not only conceptually but also perceptually, i.e. metonymy in situations without language, PART TWO aims to show that metonymy as a natural cognitive mechanism significantly operates without conscious awareness in language phenomena.

PART THREE examines metonymy in communication, metonymy in interaction, to be specific. What is examined in this part is actually on-going dialogic discourse. Metonymy in discourse is touched on in some research. Nevertheless, in most cases the discourse data have been selected mainly from written texts, e.g. a news report, or an article, which are in essence descriptive and narrative and thus static to some extent. Furthermore, most of the study on metonymy in this kind of discourse is closely related to grammar, with exceptions of Barcelona (2005a, 2005b, 2007a, 2007b), which have studied some discourse functions of metonymy, e.g. metonymy 
as guiding implicature across longer texts. Metonymy as a natural cognitive mechanism operates not merely in one individual person but in everyone involved in the interaction. Therefore, in interpersonal communication, metonymy is destined to have influence and interact between communicators. Language shapes thought and shapes the way the users think (Boroditsky, 2001, 2009; Boroditsky et al, 2003). Language influence functions especially when people are "thinking for speaking" (Slobin, 1996) ${ }^{15}$. What one has in mind in terms of language, what one hears, and what one is to reply, interact in dialogic discourse. This is a different perspective and experience from that of static discourse.

PART THREE thus aims to analyse how metonymy functions in on-going dialogic discourse. In particular, it examines the structuring and developing operation of metonymy, i.e. how metonymy contributes to structuring and developing the dialogue between the interlocutors and makes it a coherent discourse entity. It also considers how metonymy helps meaning-making, decision-making and problemsolving in the dialogue.

Besides the generally termed meaning construction or the narrowly meant sensemaking, metonymy also contributes to the discourse as an entity in terms of meaning, i.e. it makes meaning out of the local utterance and relates it to the whole dialogue or the whole scenario. In dialogic discourse, metonymy is also found to function both in the decision process of what to utter or to reply, and to operate in the problemsolving process of how to formulate and organise the utterance or the reply to the questions posed by the counterpart. Why is it that the speaker utters such metonymic expression, or organises his thinking metonymically; what influence does the metonymy exert on the listener and what kind of response is invited from the listener in the talk-turns; and what cognitive (pragmatic) strategies s/he employs in the

\footnotetext{
${ }^{15}$ In Slobin's view, thought is much richer than language; they are separable and we can do thinking without language; but once language is acquired and put to use, it will influence our thinking; we only fit our thoughts into available linguistic forms. Thinking for speaking is what we do in actual communication. It involves picking those characteristics that (1) fit some conceptualization of the event, and (2) are readily encodable in the language (Slobin, 1996, p. 76). In communication we adjust our thinking in accordance with what comes to our mind of the linguistic forms such as words, phrases, or constructions, but we may not necessarily mean what those linguistic forms indicate; we sometimes repair our utterances owing to the thinking for speaking process.
} 
responding: These are all taken into account in the analysis of dialogic discourse. The dialogic discourse data comprise real daily dialogues, broadcast interviews, and movie dialogues. The analysis shows that metonymy underlies the whole process of dialogue, making a local, discoursal contribution in terms of discourse organization and development, making a conceptual contribution in terms of thinking and reasoning, and making a strategic contribution in terms of decision-making and problem-solving. Metonymy is crucial in the mind and ubiquitous in language, nevertheless what is examined as metonymy in this analysis is not every word in the dialogic discourse, not every utterance, but those regarded as typical examples of metonymy and as serving the function for the purpose of the interaction, even though they are implicitly underlying the actual utterance in the dialogues. 
PART ONE METONYMY IN THE MIND 


\section{Chapter 2 Metonymy in the Cognitive Perspective}

Metonymy is traditionally viewed as a figure of speech in which one word or phrase is substituted for another with which it is closely related, and it is considered to involve a transfer of the name, a substitution relation between words. What is more, metonymy is generally subsumed under metaphor and taken as a special type of metaphor; thus attention has been focused on metaphor since Aristotle, and a large number of papers and books are devoted to metaphor with very little writing on metonymy specifically.

A little different from the traditional rhetorical view of word substitution, the historical semanticists borrow the figures of speech from rhetoric as procedures of semantic change, and regard metonymy ${ }^{16}$ as a mechanism of meaning extension. Historical semanticists such as Robert Thomas (1894) use metaphor and metonymy as terms to designate shifts in meaning across conceptual boundaries, metaphor based on subjective correspondences and metonymy based on objective correspondences; metaphor and metonymy are treated as semantic changes based on transfers between conceptual spheres (Nerlich, 2010).

Later on, the structuralists made a distinction between sense transfer and name transfer and viewed metonymy (and metaphor) from the perspective of the objective world, considering that metonymy involves one thing standing for another with which it is closely associated (and metaphor involves one thing standing for another with which it is similar). In this view, metonymy is a relation of contiguity in sense (Ullmann, 1962, p. 218), a transfer of meaning(Nunberg, 1995), a relation between things (Bredin, 1984), a modifier-head relation(Warren, 1999, 2002, 2006); while metaphor is a relation of similarity in sense (Ullmann, 1962, p. 212). Metonymy and metaphor form two complementary semantic change mechanisms, for contiguity is taken to include any associative relations other than those based on similarity (Ullmann, 1962, p. 212). Jakobson (1956/1971) places metonymy and metaphor on different axes, raising metonymy to the same status as metaphor and thus forming opposing poles. Jakobson views metonymy as the syntagmatic axis, which is based

\footnotetext{
${ }^{16}$ The so-called four master tropes (metaphor, metonymy, synecdoche and irony) are often reduced to metonymy and metaphor, and hereafter metonymy is often spoken of together with metaphor whether in comparison or contrast.
} 
on combination and contexture underlying the external relation of contiguity (uniting the constituents of a context), and metaphor as the paradigmatic axis, which is based on selection and substitution underlying the internal relation of similarity (linking with the code) (Jakobson, 1971a, pp. 232-233; 1971b, pp. 243-259).

Lakoff \& Johnson's seminal book Metaphors We Live By triggers a worldwide fresh interest in metaphor and metonymy from the cognitive perspective. Distinct from the traditional rhetorical view, which stresses the names, and the structuralist view, which emphasises the sense, and somewhat like the approach of historical semantics, the cognitive view of metonymy and metaphor underlines the concept. From the literature on metonymy, it could be considered that there are four main approaches to metonymy (and metaphor) from the conceptual perspective: The cognitive domain approach, the reference point/mental access approach, the blending theory, and the contingent indexical approach ${ }^{17}$.

This chapter examines metonymy in current Cognitive Linguistics, focusing on the above-mentioned cognitive approaches to metonymy, and then proceeds to argument about the widely held cognitive domain approach, discussing mapping and domain, followed by discussions on contiguity interpretation and a summary.

\subsection{Metonymy in Cognitive Linguistics}

The cognitive domain approach considers metonymy and metaphor as cognitive processes. In metonymy one domain is mapped onto another within the same domain (matrix) or ICM (idealized cognitive model), i.e. it is an intra-domain mapping; whereas in metaphor one domain is mapped onto another different domain, i.e. it is an inter/cross-domain mapping (Barcelona, 2002a; Croft, 2002; Lakoff, 1987b; Lakoff \& Turner, 1989; Ruiz de Mendoza, 1998, 2000; Thornburg \& Panther, 1997). The typical definitions of metonymy in this approach are:

1) Metonymy is a process which allows humans to conceptualize one thing by means of its relation to something else (Lakoff \& Johnson, 1980).

\footnotetext{
${ }^{17}$ These approaches are more or less related to each other, or even intertwined, especially the first two. This consideration of different approaches is put forward mainly due to the judgement that each of the views focuses on different perspective though interrelation is involved.
} 
2) Metonymy is a mapping from the source conceptual entity to the target conceptual entity in the same domain with a primarily referential purpose (Lakoff, 1987b; Lakoff \& Turner, 1989).

3) Metonymy is a domain-internal one-correspondence conceptual mapping where the matrix domain can be either the source or the target of the mapping and where the target domain is a non-central characterization of the source (Ruiz de Mendoza, 1997, 2000).

4) Metonymy is a mapping of a conceptual domain, the source, onto another domain, the target. Source and target are in the same functional domain and are linked by a pragmatic function, so that the target is mentally activated (Barcelona, 2002a).

The reference point/mental access approach sees metonymy as a conceptual reference point phenomenon providing mental access to the target concept based on the conceptual contiguity relationship (Feyaerts, 2000; Langacker, 1993; 1999; Radden, 2005; Radden \& Kövecses, 1999). The following are some typical definitions in this approach:

1) Following Lakoff (1987), Radden/Kövecses (1999) defines metonymy as:

A cognitive process in which one conceptual entity, the vehicle, provides mental access to another conceptual entity, the target, within the same idealized cognitive model (ICM).

2) Following Langacker (1993), Radden (2005) defines metonymy as:

A reference point phenomenon in which one conceptual entity, the reference point or vehicle, provides mental access to another conceptual entity, the target.

In line with the reference point/mental access view, the contingent indexical approach, which is really a revival of the semiotic view of metonymy as an indexical relation and metaphor as an iconic relation in the conceptual perspective, regards the target meaning in metonymy as a type of meaning elaboration, arguing that the metonymic relation is a contingent indexical relation while metaphor is an iconic relation (Panther, 2006). It is pointed out that this indexical relationship between the source and the target is not arbitrary like the symbolic relation between linguistic 
vehicle and its meaning, but motivated instead. In the indexical relation between the source and the target, the target concept is construed as conceptually more complex.

The conceptual blending theory is not specifically a theory of metonymy or metaphor, but a general theory of online meaning construction. However, it is also used to try to interpret metonymic phenomena. In this theory, metonymy is viewed as a conceptual blending between the source concept and the target concept, which generally involves two mental input spaces (the source and the target), a generic mental space (encompassing both the source and the target), and an emergent mental space (i.e. the blend, or blended space) (cf. Alač \& Coulson, 2004; Coulson \& Oakley, 2003; Fauconnier \& Turner, 1999; Turner \& Fauconnier, 2000).

However, as the above definitions and descriptions show, some problems accompany these approaches.

1. The reference point/mental access approach applies not only to metonymic phenomena, but also to other semantic phenomena such as active zone, facets (Geeraerts \& Peirsman, 2011; Paradis, 2004), even to metaphoric phenomena in some cases, especially in conventionalized metaphor, where a WHOLE frame of SOURCE and TARGET has been formed and the trigger of the source can automatically activate the target, i.e. conventionalized metaphor can be viewed as a mental access process in which the target concept can be automatically accessed through the source due to the high conventionality, e.g.

(1) The world is a stage.

In the perception or conceptualization of "the world", some point(s) are sensed as similar to "the stage" where people perform, and this similarity between them has been conventionalized and thus in cognition they are experienced as a WHOLE or ICM/Frame. Thus even without explicit presentation of the similarity between them, mental access can be provided from "the world" to "a stage", or the other way around. In this sense, all conventionalized metaphors can be said to obviously work metonymically. 
2. The second problem is that the reference point/mental access view of metonymy seems easily to wrongly lead to the referential perspective of metonymy. It is true that metonymy can basically refer to things, it can be used to understand as well, and can be predicative or propositional and illocutionary (cf. Panther \& Thornburg, 1998; Panther \& Thornburg, 1999; Ruiz de Mendoza, 1997; Ruiz de Mendoza \& Díez Velasco, 2003; Ruiz de Mendoza \& Pérez Hernández, 2001; Stallard, 1993; Sweep, 2009; Thornburg \& Panther, 1997; Warren, 2002). For example:

(2) Could you please clear the table?

It means to clear what is on "the table" such as the dishes, forks and knives; however, there is no reference shift here, because "table" carries its own denotation and cannot refer to other things. The same applies to "clear the dishes", because it is the verb "clear" here that determines the event/process, i.e. the metonymic understanding (cf. Sweep, 2009). ${ }^{18}$ This sentence is also a metonymy-based indirect request, i.e. illocutionary metonymy (QUESTION FOR REQUEST, ABILITY (POTENTIALITY) FOR ACTUALITY).

In addition, Ruiz de Mendoza (1997) points out that metaphors can also be used referentially, and referentiality seems to be the basic property of all tropes rather than that of metonymy alone, e.g.

(3) The pig is waiting for his check.

Here "the pig" is referred to a particular unpleasant customer who kept harassing the waitress (cf. Ruiz de Mendoza, 1997, p. 164).

Therefore, the mental access/reference point view is more likely to be a common phenomenon with all tropes.

The mental access/reference point view of metonymy discloses the function of the source providing mental access to the target, avoiding the issue of distinguishing

\footnotetext{
${ }^{18}$ The metonymic understanding of "Clear the table" is determined by the verb "clear"; in this use, "Clear" can only be followed by a word with the meaning of an area as a whole, e.g. "clear the bottle" means "remove all staff out of the bottle", with the bottle as the whole area to clear; and "clear the village" also limits to the area of the village. That is, "clear the table" is not as same as "clear the dishes/silverware/glasses".
} 
between metaphor and metonymy. However, the question why the source can provide access to the target is not successfully, at least not convincingly, unanswered. Although certain principles of human cognitive salience, e.g. human > non-human, whole > part, concrete > abstract, visible > non-visible are proposed (Langacker, 1999), actual metonymies do not necessarily follow this order. For example, FOOD FOR CUSTOMER WHO ORDERED IT and DRESS FOR PERSON WHO WEARS IT in examples (11) and (12) in Section 2.2.2 below do not follow these salience principles. The question of how the mental access can take place or be triggered is left unanswered as well. ${ }^{19}$ Thus, this reference point/mental access view of metonymy is not unproblematic (Radden \& Kövecses, 1999).

3. The contingent indexical approach treats metonymic relations as indexical. Nevertheless, some metonymic relations may be iconic as well, e.g.

(4) This is Eve. (Showing a picture of a girl)

(5) He has his father's eyes.

These two are examples of metonymy with iconic rather indexical relations (Warren, 1998, pp. 304-306).

4. The conceptual blending theory regards blending as ubiquitous, thus metonymy surely involves blending. A close scrutiny finds, nevertheless, that blending is actually enabled by metonymy. Metonymy plays a crucial role in blending, as pinpointed by Coulson \& Oakley (2000, p. 187; 2003, p. 52 ). The following example can help make sense of this point:

(6) He has got a Picasso in his den. (cf. Alač \& Coulson, 2004, pp. 23-24)

This metonymy of PICASSO FOR HIS ART WORK involves two input mental spaces. One is Pablo Picasso's person space, and the other is Picasso's art space. In Picasso's person space, there are many elements which act as reference points to Pablo Picasso as a person, among which is Picasso as an artist, which can further act

\footnotetext{
${ }^{19}$ Radden \& Kövecses (1999) and Kövecses \& Radden (1998) propose "metonymy-producing relationships", and Barcelona (2002a) proposes "pragmatic function (strong experiential link)" for the possibility of metonymy in cognition. But these relationships or experiential links need empirical evidence, and cognitive science and neuroscience might be the best resort.
} 
as a reference point to other concepts of art such as Picasso's techniques in art, Picasso's role in art history. In Picasso's art space, there is at least an element which acts as a reference point to the particular work of art mentioned in this example. And then these two inputs combine to yield a blended space, i.e. a Picasso. If the generic space has to be pointed out, it is "Picasso as a human", which encompasses the two input spaces. In this blending process, it may be noticed that one element is conceptually selected as a reference point to the whole space, e.g. a particular art work is picked up for Picasso's art space; Picasso as an artist is picked up for Picasso as a person. The conceptual blending theory does not explain this PART FOR WHOLE phenomenon that takes place right before the blending occurs; however, this PART FOR WHOLE cognitive selection is typically metonymic. Thus analysed, it can be clearly seen that when the conceptual blending theory tries to explain metonymy, it conceptually needs metonymy to interpret the process before the blending takes place, and that might be why Coulson \& Oakley (2000, 2003) announce that metonymy is crucial in blending. Therefore, the blending theory is deemed not to give a successful interpretation of metonymy. Otherwise, the theory falls into circular reasoning. On the one hand, Coulson \& Oakley pinpoint the significance of metonymy in blending; on the other hand, they intend to interpret metonymy with the blending theory which itself involves metonymy.

Another problem is that it is artificial to divide the source into several input spaces in analysis, e.g.

(7) Finland called. They sent their love. (Warren, 2004)

In this example, "Finland" metonymically means "the relatives living in Finland". In accordance with the blending theory, FINLAND FOR THE RELATIVES LIVING THERE might involve two inputs, one is "Finland as a country", and the other is "Finland as the residence of the relatives". But why and how to divide Finland into such input spaces seems to be a problem. If there is any rule to follow in the division, e.g. country - people living there - relatives among them, it is metonymically-related, i.e. metonymy is the precondition of the blending. Hence it seems that blending is an operation after metonymy. 
Moreover, since blending is ubiquitous, it applies to metaphor as well. Thus it seems not be able to fruitfully tell metonymy from metaphor either.

The above analysis suggests that the reference point/mental access approach, the blending theory, and the contingent indexical approach cannot successfully distinguish metonymy from metaphor. Even though these approaches seem to have pointed out the working mechanism of metonymy; this mechanism proves to be shared by both metonymy and metaphor. It could be that the blending theory cannot satisfactorily explain metonymy as a cognitive model because of its need of metonymy in the interpretation of metonymy. Of the cognitive views, the cognitive domain approach is the most widely adopted and employed in cognitive analysis of language, for this approach seems to allow an obvious distinction between metonymy and metaphor. However, under close scrutiny, this approach appears to fall into the same trap as the traditional rhetoric "closely related" view and the structuralist "contiguity" view. This approach is examined in the following section.

\subsection{The cognitive domain approach}

Although the cognitive domain view accounts for both metonymy and metaphor, distinguishing metonymy from metaphor actually creates the greatest challenge for it. Since both metonymy and metaphor are mappings involving the conceptual domain in the cognitive domain view, the key terms "mapping" and "domain" have to be interpreted and clarified in order to make a clear distinction between them.

\subsubsection{About mapping}

Fauconnier (1997) regards mapping as a one-to-one correspondence relation between two sets. Initially Barcelona (2003b) regards mapping as a superimposition of one structure onto another, but later Barcelona (2003a) views mapping as a perspective imposition, i.e. the target domain is understood from the perspective imposed by the source. Barcelona (2000c, 2003b) believes mapping is also an essential property of metonymy. Nonetheless, Ruiz de Mendoza (1997, 2000) thinks that relying on domain-internal and domain-external mappings in distinguishing metonymy from metaphor is rather difficult unless these two mappings can be determined unequivocally, and suggests that the nature of mapping should be reconsidered. $\mathrm{He}$ 
then takes metonymy as a one-correspondence mapping between domains in the same domain matrix; he sees metaphor as both a one-correspondence and a manycorrespondence mapping between domain matrices. This view, nevertheless, is reviewed and shown by Panther (2003) to be not generally true; the viewpoint is over-generalized.

Mapping, in its original sense, is a substitution relation ${ }^{20}$. Some scholars, e.g. Barcelona (2011, p. 12), understand mapping in a narrower sense as the projection of one structure onto another, i.e. the source imposes (some of) its internal elements, traits, and properties onto their counterparts in the target; the projection proceeds from a part onto another part of the domain, from a whole onto a part and vice versa, involving no structural similarity or functional or relational equivalence; hence metonymy involves an asymmetrical mapping, whereas metaphor involves a symmetrical mapping. But metaphor is in fact only a partial mapping, for the "metaphorical structuring involved is partial" (Lakoff \& Johnson, 1980, p. 13). In Lakoff's original use of mapping, it meant mathematical correspondence. And later he converted mapping to mean projection, i.e. mapping is transferred to projection from the original or essential meaning of correspondence.

Since it was felt that mapping was not used to mean correspondence but projection and it was not the right term to characterize metonymy, why not use project or projection explicitly in the definition of metonymy? If both metonymy and metaphor constitute projection, what distinguishes these two kinds of projection? Otherwise the confusion would remain. In addition, as Brdar-Szabó and Brdar (2011) point out, since metaphor and metonymy are taken as very different processes, why do they still share the same term "mapping" in their definitions? In this sense, it might be better to consider metonymy not as a mapping by nature. In "She is my joy", it is not possible to tell what is mapped onto what. Besides, mapping does not describe exactly the actual contiguity taking place in metonymy and the similarity in metaphor. Thus, Ruiz de Mendoza (2000, p. 130) says that in metonymy the structural relations which hold in the source can hardly be mapped onto the target since source and target stand in an inclusion relationship. Therefore mapping in metonymy and mapping in metaphor are not fruitfully distinguished. The metonymic

${ }^{20}$ By this sentence, I mean the original meaning as used in mathematics. 
cognitive process is quite different from the metaphoric cognitive process. If they were not, it would not be necessary to have metaphor and metonymy as opposite poles or as a continuum.

\subsubsection{About domain}

In respect of domain, Langacker (1991, p. 547) views it as any coherent area of conceptualization relative to which semantic structures can be characterized (including experience, concept or knowledge system). Barcelona (2003b, p. 230) views domain as a cognitive encyclopaedic domain, i.e. including all entrenched knowledge about the domain a speaker may have. Thus conceptual domain is the structured form of the encyclopaedic knowledge or knowledge network about an entity that a person holds. Croft (2002, pp. 166-194) regards domain as a semantic structure that functions as the BASE for at least one concept PROFILE (typically, many profiles), e.g. "circle" (base) and "arc" (profile). He puts forward base domain, domain matrix and domain highlighting. Base domain is the domain immediately presupposed by the profiled concept. Domain matrix is all the domains a concept may presuppose at once, e.g. HUMAN BEING: Physical objects, Living things, Volitional agents, etc. Domain highlighting refers to the phenomenon that makes primary a domain that is secondary in the literal meaning. Thus Croft uses "domain matrix" to replace "the same domain", and suggests that metonymy is a conceptual domain highlighting phenomenon of a sub-domain within the domain matrix, whereas metaphor is a conceptual domain mapping between two independent domains which do not form a domain matrix. Croft offers the following example:

(8) Proust is tough to read.

In this example, Proust as a person (literal meaning) is the primary domain, works of Proust as a writer is the secondary domain. This example is highlighting the secondary domain within the domain matrix of Proust as a person.

But domain highlighting applies to both metonymy and metaphor. Lakoff/Johnson (1980, p. 10) point out "metaphorical highlighting and hiding" in Metaphors We Live By. They write that "a metaphorical concept can keep us from focusing on other aspects of the concept that are inconsistent with that metaphor." It means that 
metaphor involves domain highlighting as well. Ruiz de Mendoza (1997, 2000) also contends that domain highlighting is applied to metaphor. He gives the following examples:

(9) John is a brain.

In this case, intelligence of John is highlighted via the metonymic use of "brain".

(10) John is a lion.

In this example, courage is highlighted both in the source (lion) and the target (John).

Alternatively, Ruiz de Mendoza and Díez Velasco (2003), and Ruiz de Mendoza \& Pérez Hernández (2001) propose domain expansion (i.e. source-in-target metonymy) and domain reduction (i.e. target-in-source metonymy). While other scholars do not distinguish cognitive models and domains (e.g. Lakoff, 1987b; Radden \& Kövecses, 1999), Ruiz de Mendoza and Campo (2002) make a distinction between cognitive models and domains; they combine these two in order to explain the notion of matrix domain. In Ruiz de Mendoza and Campo's view, a cognitive model is a conventionally structured semantic configuration and a domain is a frame of reference for the activation of part of the information of a cognitive model. Take Lakoff's (1987a, 1987b) "mother" analysis as an example. According to Lakoff, "mother" as a concept has at least such cognitive models as the BIRTH model, the NURTURANCE model, the MARITAL model, the GENETIC model, and the GENEALOGICAL model. These cognitive models are the BASE in Ruiz de Mendoza and Campo's view, i.e. each of them constitutes a matrix domain; simultaneously, the conceptual structure of each of these cognitive models acts as the PROFILE against other base domains which are activated by the notion "mother". For example, the BIRTH model can be profiled against many base domains such as the "procreation" domain characterized by the existence of sexual interaction, partners, etc, the "pregnancy' domain including time of pregnancy, cravings, a foetus, etc, the "birth" domain consisting of hospital, birth wards, the midwife, etc; each of these domains serves as a frame of reference for the activation of relevant 
information contained in a cognitive model (Ruiz de Mendoza \& Otal Campo, 2002, p. 60).

However, "domain" per se as a key term, (whether defined as structured encyclopaedic knowledge or a reference frame for activation of relevant information in a cognitive model), is far from determinate. Some scholars think the term "domain" is rather vague and it is impossible to make a clear-cut demarcation between boundaries, since the range of domain varies from person to person (Dirven, 2002; Feyaerts, 1999; Riemer, 2002). Dirven (2002: 87) thinks that this term proper needs further clarification before it is used to interpret other phenomena. Panther (2006) argues that since the notions such as "domain", "single domain", "sub-domain", "separate domains", which are often used as definitional criteria for distinguishing metonymy from metaphor, are cover terms for heterogeneous concepts and conceptual relations, and they are unreliable in distinguishing metonymy from metaphor. Feyaert (1999) points out that it is often an arbitrary intervention to draw distinct boundaries around a domain (matrix), which definitely results in a methodological problem, and "domain" or "domain matrix" is a notion too malleable to serve as a criterion of defining metonymy. Taylor (2002b) also contends that, since domains typically overlap and interact, it is an error to suppose that domains constitute strictly separated knowledge configurations. In addition, Panther (2006, p. 160) spells out that Croft's conception that "metonymy makes primary a domain that is secondary in the literal meaning" is not compatible with the idea that metonymy is a domain - sub-domain or sub-domain - domain relationship.

What is more, an entity can be said to fall in different domains according to the viewer's perspective or different situation. For instance, "110" as the police emergency phone number in China can be said to be in the domain of Number (110 as a number), Telecommunication (110 as a phone number), Digital system (110 as communicative system or platform), Police force (110 as collective police), People (110 as a policeman), Vehicle (110 as a police car), Emergency (110 extended as any emergency situation), etc. Furthermore, which domain applies in a specific situation is also a problem. Ruiz de Mendoza and Campo (2002, p. 54) provide the following examples: 
(11) The ham sandwich has left without paying.

(12) The fur coat has left without paying.

These two examples are utterances occurred in a restaurant. But it is obvious that it is not the RESTAURANT domain that determines the metonymic process in both sentences. If the restaurant model or domain plays the role in (11), i.e. FOOD FOR CUSTOMER WHO ORDERED THE FOOD, it is absolutely not in (12). Instead, it is DRESS FOR PERSON WHO WEARS IT, which is not in the RESTAURANT model/domain, at least not immediately. ${ }^{21}$

Thus on different occasions, a particular entity would be in different domains, and even when it is clearly in a certain domain according to the folk knowledge, it is not always necessarily this domain that determines the metonymic process in utterances. What is more, it is often hard to decide that an entity is definitely of a certain domain.

And another problem in the application of domain in the analysis of metonymy in the literature is that domain seems to be made a Jack of all trades and used everywhere as analysis needs. For example, time and space are regarded as different domains (the primary domains (Grady, 1997)), while an event which inevitably involves time and space is also considered as a domain such as dining in a restaurant, or taking a taxi. It seems domain has become an omnipotent word.

Combining the above, the problems with domain are:

1) Unclear boundaries (i.e. the range). Since domain is structured encyclopedic knowledge, it is open-ended and may vary from person to person.

2) Tough domain decision (i.e. the level). In Croft (2002), domain is raised to a higher abstract level and thus hierarchies of domain are established. Since domains have hierarchies and are interrelated with each other, a super-domain can always be found and two domains will always have at least one domain in common that encompasses them, e.g. a man and a pig

\footnotetext{
${ }^{21}$ The notion of domain is confused quite often in the study of metaphor and metonymy. Sometimes it means the basic knowledge categories such as TIME and SPACE, and on other occasions it is used to indicate encyclopaedic knowledge frame or scenario such as RESTAURANT and SHOPPING. These cases suggest the indeterminacy of domain as a term.
} 
might be said to either be in a domain matrix (ANIMATE) or in different domains (HUMAN vs. ANIMAL); it is often difficult to name or tell one domain from another.

3) Changeable perspectivization. That is, the relationship of domain - subdomain depends on the perspective of the viewer; a domain - sub-domain relation may be also viewed as a sub-domain - domain relation instead (Panther, 2006), e.g.

(13) The ham sandwich is waiting for his check.

The ham sandwich can be either a domain or sub-domain depending on how the situation is viewed. If it is viewed from the perspective of food, the ham sandwich is the domain, the customer is the sub-domain; if it is viewed from the other way round, then a different domain---sub-domain relation will be reached, e.g. if it is viewed from the perspective of customer, the customer is the domain, and the food is the sub-domain.

4) Same domain metaphors. In fact, it is also likely to have metaphors which obviously involve the same domain, e.g.

(14) My pet tiger is a lion. (Panther, 2006)

Tiger and lion are obviously in the same domain according to the folk taxonomy of domain (Barcelona, 2003b, p. 231).

So from the above analysis, it is clear that the terms "mapping" and "domain" in the definitions of, and in the distinction between, metonymy and metaphor in the cognitive view are somewhat problematic. This kind of view might be operational, but it seems unable to solve the problem at the root. It is a terminological paradox; the problem with "contiguity" and "similarity" still exists. In order to have a clear conception of metonymy and how it works conceptually, it is better first to examine what counts as metonymy so far in terms of "contiguity", and then to check what cognitive operation underlies it through analysing its linguistic representations. 


\subsection{Interpreting "contiguity"}

From the very beginning, metonymy was considered as a category of relation constituted by many types, e.g. relations between PART and WHOLE, between GENERIC and SPECIFIC, as related by the terms "closeness" in traditional rhetoric or "contiguity" in structuralism or "intra-domain" in Cognitive Linguistics. The above analysis suggests that that the change of terms leaves the relation problem unresolved. Since the definitions are problematic, and "contiguity" plays a crucial role in metonymy, it is better to observe what counts as metonymy and see what relations between the source and the target count as metonymy, i.e. examine how "contiguity" is interpreted and described in the literature.

According to Arata (2005), the first datable mention of metonymy is by Trypho of Alexandria in the first century B. C.. In his De Tropis metonymy is described as "a word which means the synonym starting from the homonymy" (Rhetores 3.195, 2026. see Arata (2005, p. 57)). Trypho II in $1^{\text {st }}$ century A. D. defined metonymy as "a part of speech which is imposed on a given thing in a literal sense, but which signifies another given thing according to a type of relationship" (see Arata (2005, p. 57)). And then in Rhetorica ad Herennium, metonymy was considered as "the figure which draws from an object closely akin or associated an expression suggesting the object meant, but not called by its own name" (see Bredin (1984, p. 52) and LaVelle (2007, p. 132). Although what was metonymy was somewhat implicit in these definitions, the examples given or the types of metonymy listed were explicit. It is also noticed that metonymy went from word naming to a figure of speech during this period. Fontanier (1830) categorised metonymy as "tropes by correspondence consisting in the designation of an object by the name of another object, with both constituting a completely separate whole, but one owing its existence or mode of being to the other". Alexander Bain (1871) classified metonymy as "a figure based on the mental association of contiguity in general and on accompaniment in particular" (see VaLelle, 2007, pp.137-138). From these traditional views of metonymy some divergent opinions of metonymy have been derived. Some stress the naming aspect, e.g. in rhetoric; some emphasise the semantic aspect, i.e. in intralinguistic relations such as the Group $\mu$ (1970) (see Bredin (1984) and Nerlich (2010) ), Jakobson (1971b), Ullmann (1962), and Warren (1998, 2006); some focus 
on the extralinguistic aspect such as between objective entities, e.g. Bredin (1984) and Seto (1999), and between conceptual entities, e.g. Blank (1999) and Koch (1999, 2001). Although the literature presents some different views of metonymy in terms of definition and the underlying mechanism, some types of metonymy are shared. For instance, the Ancient Greek rhetors classify the following as metonymy (see Arata, 2005, pp. 58-61):

1) Saying the name of a divinity to mean the name of his attributes and vice versa, e.g. "Hephaestus" signifying "fire"

2) Saying the thing that is contained by the name of the thing containing it, e.g. "The house" signifying "things which are in the house"

3) Saying the thing that contains by the name of the thing that is contained, e.g. "Head and shoulders" signifying "helmet and shield"

4) Saying the name of a place to signify the name of its inhabitants, e.g. "the earth" signifying "men"

5) Saying the name of the instrument to signify the name of the activity, e.g. "bow" signifying "archery"

6) Saying the name of the thing that has a quality to signifying the name of the quality, e.g. "rush" signifying "obscurity"

7) Saying the name of the cause to signify the name of the effect, e.g. "terrible fear" for "the terrible thing that produces fear"

The list of relations such as these that are categorised as metonymy is far from exhaustive. The following instances are regarded in the literature as common relations in metonymy (see, e.g. Arata, 2005; Barcelona, 2000b; Dirven \& Pörings, 2002; Kövecses \& Radden, 1998; Lakoff, 1987b; Lakoff \& Johnson, 1980; LaVelle, 2007; Panther \& Radden, 1999; Radden \& Kövecses, 1999):

AGENT FOR ACTION (and vice versa), e.g. "butcher" the cow; "author" a book.

ACTION FOR INSTRUMENT (and vice versa), e.g. She "pianoed" and sang last night. "Shampoo" the hair.

ACTION FOR MEANS (and vice versa), e.g. The passengers were "bussed" back to the airport. He "sneezed" the tissue off the table. 
MEMBER FOR CATEGORY (and vice versa), e.g. "aspirin” for "pain-killer"; "pill" for "contraceptive medicine".

CONTAINER FOR CONTENTS (and vice versa), e.g. The "kettle" is boiling. Give me the "salt".

CONTROLLER FOR CONTROLLED, e.g. "Nixon" bombed Hanoi. A "Mercedes" rear-ended me. The "buses" are on strike.

FUNCTION FOR ORGAN (and vice versa), e.g. He is a "brain".

INSTRUMENT FOR POSSESSOR, e.g. The "saxophone" is sick.

OBJECT FOR MATERIAL CONSTITUTING THE OBJECT (and vice versa), e.g. "steel" for "sword"; "wood" for "forest".

PART FOR WHOLE (and vice versa), e.g. There are some new "faces" in the office. "England" for "Great Britain"; "America" for "United States"; "rear" for "bum".

PLACE FOR INSTITUTION (and vice versa), e.g. "Wall Street" has long been bored with the US budget deficit. "Hollywood" is not what it used to be.

PLACE FOR EVENT (and vice versa), e.g. "Watergate" changed our policy. POSSESSOR FOR POSSESSION (and vice versa), e.g. The "BMW" is my friend.

PRESENT FOR FUTURE, e.g. I "am finishing" the job.

PRESENT FOR HABITUALITY, e.g. He "plays" soccer every Sunday.

STATE OF BEING FOR RESULT, e.g. That is "excellent".

PRODUCER FOR PRODUCT, e.g. He's got a "Picasso". A "Ford".

This kind of relationship is found to be underlying the conversion of word-class, e.g. conversion between verb and noun (Dirven, 1999), and indirect speech acts 
(Thornburg \& Panther, 1997), in other grammatical aspects (Langacker, 2004; Panther \& Thornburg, 2000, 2002, 2003a; Panther et al., 2009), and in making pragmatic inferences (Panther \& Thornburg, 2003b). Since the relations between the source and the target in metonymy cannot be exhaustive, and "contiguity" is a "wishy-washy notion" (Koch, 1999, p. 145) and a "convenient but unrevealing metaphor" (Bredin, 1984, p. 47), scholars endeavour to classify those relations generally taken as metonymy and discover what counts as the relationship of "contiguity". Some assume that metonymic relations take place between things and explore the relations between objects. For example, Bredin (1984) takes metonymy as simple extrinsic relations, i.e. non-dependent relations between objects, which is a structural relation within a thing; and metaphor, which is a dependent relation between things. In Bredin's view, cause and effect are in a simple relationship because there is no property possessed by both a cause and its effect. Seto (1999, 2003) treats metonymy as the spatio-temporal contiguity between objective "entities", thus distinguishing metonymy from synecdoche, which is instead a semantic inclusion between two "categories".

In Cognitive Linguistics, synecdoche is not taken to have a position distinct from metonymy and is treated under the umbrella of metonymy, since there is no conceptual difference between metonymy and synecdoche (they work in the same way) and no cognitive significance in excluding synecdoche in the cognitive study of metonymy. See e.g. Koch (1999) Lakoff (1987b), , Peirsman \& Geeraerts (2006). Moreover, Seto's distinction between entity and category cannot gain any cognitive support, even though he views them from the cognitive perspective, because an entity, whether concrete as spatial or abstract as temporal, is conceptually a category. Categorization is an internal feature of human cognitive process, for which humans have an insatiable appetite (Trenholm \& Jensen, 2004, p. 144). The capacity to categorize is the most fundamental of human capacities (Lakoff, 1987b, p. 8). Bredin's argument also seems unsuccessful in expounding the "contiguity" relationship; his dependent and non-dependent distinction is no different from Ullman's (1962, p. 212) similarity and non-similarity between metaphor and metonymy. 
Ullman (1962, pp. 218-210) is one of those scholars who explores the contiguity relationship by virtue of sense. He thinks that the underlying relations in metonymy are mainly spatial, temporal, and causal. Spatial relationship is physical proximity, e.g. from Latin "coax" (hip) to French "cuisse" (thigh) is a spatial relation. Temporal relationship is temporal succession, e.g. French "veille" (vigil) with the present meaning "eve, preceding day". Causal relationship is a relation in which one causes another as a result, e.g. ACTION FOR RESULT. Ullman also mentions the PARTWHOLE relation, but does not relate it to the above three relations. Moreover, his classification is not clear-cut and excludes some obvious types of metonymy, e.g. possessive relations, and hierarchical relationship between the source and the target (see also LaVelle, 2007, pp. 152-153).

Another scholar viewing the contiguity relation from the sense aspect is Warren (see e.g. Warren, 1995, 1998, 1999, 2002, 2004, 2006). She distinguishes five metonymic relations: Composition (e.g. SOURCE-RESULT, SUBJECT-SET, and MATERIALOBJECT), possession (e.g. PART-WHOLE), location in time and space (e.g. PLACE/TIME-OBJECT, PLACE-EVENT), causation (e.g. CAUSE-EFFECT), and representation (MODEL-REPRESENTATIVE). All these are concomitant relations between entities, i.e. referential, compared with those between propositions, i.e. propositional. Warren further proposes that all these metonymic relations are in fact a modifier-head relation with the head implicit and the modifier explicit in the linguistic expressions. For instance,

(15) Mary is Cinderella in the play. ("Cinderella" for "the person representing Cinderella") (Warren, 1999, p. 131)

(16) That is the house the yellow Volvo lives in. ("the yellow Volvo" for "the one who has the yellow Volvo") (Warren, 2006, p. 19)

As regards referential metonymy, Warren's description is compelling. However, due to the restriction of her research in referential metonymy to the (syntactic-)semantic aspect, a large part that is regarded as metonymy in Cognitive Linguistics such as proposition metonymy and speech act metonymy (Panther \& Thornburg, 1999, 2003b; Thornburg \& Panther, 1997) is ignored. 
Besides the contiguity of objective entities and the contiguity of sense, metonymy is also examined as conceptual contiguity. In the Cognitive Linguists' view, the "contiguity" in metonymy is not between words, nor between senses, nor between objects in the real world, but between concepts. Blank (1999) proposes two contiguity relations, i.e. co-presence in time and space and succession in time, and they are expected to encompass all possible metonymies. Yet it is not stated why one element rather than other can be conceptually viable to form a metonymy when they are co-present or in successive order.

Koch $(1999,2001,2004)$ treats "contiguity" as the relation existing between elements of a prototypical conceptual/perceptual frame or between the frame as a whole and its elements. In his view, "domain", which is often used to denote contiguity-based frames as well as taxonomic hierarchies in an undifferentiated manner, and "ICM" (Idealized Cognitive Model) (Lakoff, 1987a), which is also an ambiguous term, should be avoided; instead he opts for "frame" in the general sense, which comprises "scene", "scenario", "script". ${ }^{22}$ Koch (2004, p.7) sees the advantage of "frame" in expressing a notion that is perfectly compatible with the notion of "contiguity". In his view, a frame unites and structures encyclopaedic expectations that are based on concepts-connecting contiguity; the contiguity relations connect elements of a frame with each other as well as one element to the frame as a whole. Koch thus takes contiguity as a conceptual relation within a frame, and pushes "frame" to the frontline. Although "frame" is borrowed from psychology, it seems that this term itself is not well defined, and the problems with "domain" seems to apply as well.

From the conceptual perspective, Kövecses \& Radden (1998) and Radden \& Kövecses (1999) conclude that the conceptual contiguity relations underlying metonymies are actually WHOLE-PART (in an ICM) and PART-PART (in an ICM). These two relations are assumed to be able to provide mental access from the source to the target within the same ICM. The ICM concept is supposed to include people's encyclopaedic knowledge of a particular domain and the cultural models as well. They suggest the configuration of the Whole ICM and its parts involves at least:

${ }^{22}$ Barcelona (2011) also expresses his preference for frame. 
Thing-and-part ICM (e.g. WHOLE THING FOR A PART OF THE THING and vice versa)

Scale ICM (e.g. "speeding" in "Henry is speeding again"; "old" in "How old are you")

Constitution ICM (e.g. OBJECT FOR MATERIAL CONSTITUTIING THAT OBJECT and vice versa)

Complex event ICM (e.g. PART OF AN EVENT FOR THE WHOLE EVENT and vice versa, for instance, "standing at the altar" for "wedding ceremony"; "behind the wheel" for "driving"; "put your hands together" for "clap/applaud") Category-and-member ICM (e.g. A CATEGORY FOR A MEMBER OF THE CATEGORY and vice versa)

Category-and-property ICM (e.g. CATEGORY FOR DEFINING PROPERTY and vice versa, for instance, "blacks" for "black people")

In the case of PART-AND-PART configuration, Kövecses \& Radden (1998) suggest:

Action ICM (e.g. INSTRUMENT FOR ACTION, AGENT FOR ACTION and vice versa, OBJECT INVOLVED IN AN ACTION FOR THE ACTION and vice versa (to "blanket" the bed; one "bite"), RESULT FOR ACTION and vice versa (a deep "cut"), MANNER OF ACTION FOR THE ACTION (to “tiptoe"))

Perception ICM (e.g. PERCEPTION FOR THING PERCEIVED and vice versa ("sight" for "thing seen"))

Causation ICM (e.g. CAUSE FOR EFFECT and vice versa)

Production ICM (e.g. PRODUCER FOR PRODUCT)

Control ICM (e.g. CONTROLLER FOR CONTROLLED and vice versa)

Possession ICM (e.g. POSSESSOR FOR POSSESSED and vice versa)

Containment ICM (e.g. CONTAINER FOR CONTAINED and vice versa ("glass" for "wine"))

In their view, the conceptual relations between the source and the target in metonymy are possible happenings within the same ICM (they term them as Metonymy-producing relationships), and can be said to be the following two: One is WHOLE-PART, and the other is PART-PART. By the same token, Ruiz de Mendoza (1997, 2000) advocates WHOLE-PART (i.e. PART FOR WHOLE and 
WHOLE FOR PART) relationship, but rejects the PART-PART relation. He argues that underlying metonymies is either a source-in-target relation or a target-in-source relation. Nevertheless, these WHOLE-PART views, unlike Blank's co-presence and succession, need to address what counts as the WHOLE and to what degree the source and the target fall into a PART-WHOLE relation, e.g.

(17) She is a pretty face here.

(18) John is a brain.

Examples (17) and (18) are generally said to be in the PART-WHOLE metonymy. In (17) "face" is related with "beauty", and in (18) "brain" is related with "intelligence" (Ruiz de Mendoza \& Otal Campo, 2002, p. 55). In the "beauty" sense, "face" is related to its appearance; whereas in the "intelligence" sense, "brain" is related to its function. Even though face and brain are both parts of a person, it depends on the situation whether face and brain are referred to and conceptually constructed on the same person. These examples, together with examples (11) and (12), urge the consideration of the immediate and non-immediate categories, i.e. category/domain boundaries, even when they are in the same domain (in the term of the cognitive domain approach.

Thus the WHOLE-PART views are subject to their operable definition of domain or ICM, whose inaccuracy has been argued in the previous part.

All scholars working on metonymy have attempted to interpret the so-called "closeness", or "contiguity" of metonymy. This becomes so challenging that some scholars even adopt a non-similarity interpretation of contiguity. For example, Ullman (1962, p. 212) and Feyaerts (2000, p. 63) treat contiguity as any association other than similarity. However, Warren (1998, pp.304-305) has shown that metonymy can be based on similarity as well. Thus the similarity and non-similarity distinction between metaphor and metonymy is not factually viable. In order to interpret the "contiguity" of metonymy, Cognitive Linguistics takes metonymy as a cognitive process or model, proposing metonymy as an intra-domain mapping or a reference point/mental access, which, as analysed earlier, still leaves the difficulty unresolved, at least not convincingly resolved. Since the "closeness", or rather, 
"contiguity" or "domain" description does not clarify the underlying operation of metonymy nor classify the types of metonymy, metonymy is taken as a prototypical category (Peirsman \& Geeraerts, 2006).

In Peirsman and Geeraerts's view, "contiguity" is not a unitary concept but a prototypical concept, which can occur in the spatial and material domain, in the temporal domain, in actions, events and processes, and in assemblies and collections. Spatial part-whole contiguity is treated as the core of the category, extending to other forms of contiguity in terms of strength of contact (going from part-whole containment with physical contact to adjacency without contact), boundedness (bounded part-whole relationship developing towards unbounded), and domainhood (from the spatial to the temporal, to the spatio-temporal, and to the categorical domains). This argument suggests that Peirsman and Geeraerts still hold the PARTWHOLE view of metonymy as do Radden and Kövecses and Ruiz de Mendoza. The only difference is that Peirsman and Geeraerts consider this PART-WHOLE relation as projected from the spatial domain to the temporal, to the categorial domains, from bounded aspect to unbounded aspect, from physical contact to non-physical contact; i.e. the PART-WHOLE relation is three-dimensionally radiating. Barcelona (2011) nevertheless considers this prototypical category as merely one part of the categories of schematic metonymies in terms of metonymicity, other categories include Purely Schematic, Typical, and Simply Typical (see also Barcelona (2003b)).

Much effort has gone into interpreting and describing "contiguity", not much has been achieved that really convinces. Nevertheless, the literature still offers some clarification of the metonymic phenomenon:

1) It is not a conceptual substitution relation, although the source syntactically stands in the place of the target. For example, Warren discovered that referential metonymy involves a modifier-head relation not a stand-for relation. 
2) Metonymy can be propositional and illocutionary as well as referential; e.g. Warren (1999, 2002, 2006), Thornburg \& Panther (1997), Panther \& Thornburg $(2002,2003 b){ }^{23}$

3) Metonymy is not merely based on non-similarity relations, it is not indexical either in terms of semiotic contrast between indexical and iconic, i.e. the long held view of metaphor as based on iconic resemblance and metonymy as based on indexical contiguity. Instead metonymy can also be based on similarity between the source and the target as well as indexical. For example, Warren (1998, 1999):

(19) This is Eve. (Showing a picture of a girl) (Warren, 1998, pp. 304-305)

4) Metonymy is regarded as a cognitive process in which the target concept can be accessed via the source through some relationships, e.g. mapping, reference point/mental access.

5) This process is supposed to take place within a certain range, e.g. frame, domain, ICM, though it is hard to define.

6) This process is supposed to be a prototypical category with part-whole relation going from spatial to temporal, and to categorical domains.

7) This process is supposed to occur conceptually, i.e. conceptual metonymy (Dirven, 2002; Fauconnier \& Turner, 1999; Lakoff, 1987b; Panther \& Radden, 1999; Panther \& Thornburg, 2003b).

8) This process has to be licensed by some strong experiential links: Pragmatic function, privileged connections or metonymy producing relationships (Barcelona, 2011; Kövecses \& Radden, 1998).

Thus realized, an integrated definition of metonymy is provided by Barcelona (2011, p. 52) as follows:

Metonymy is an asymmetric mapping of a conceptual domain, the source, onto another domain, the target. Source and target are in the same functional domain and are linked by a pragmatic function, so that the target is mentally activated.

\footnotetext{
${ }^{23}$ Some scholars, e.g. Geeraerts \& Peirsman (2011), still take referential shift as the definitional property of metonymy, but there are many non-referential metonymies in language and even metonymies without a referential shift (Sweep, 2009), which will be illustrated in PART TWO: METONYMY IN LANGUAGE.
} 
To put it simply, what counts as metonymy in Cognitive Linguistics is the following:

A and B are within a WHOLE (i.e. in the same ICM/Frame/Domain)

$\mathrm{A}$ and $\mathrm{B}$ are functionally linked in experience

A activates B (B is mentally accessed while A is mentioned/projected)

Given such understanding, some questions may arise, e.g. since metonymy is enabled by strong experiential link, how can sense be made of the experiential link? Or in other words, is there anything sensible that makes this process happen? Does this operation happen in such basic cognitive activities of infants beyond language influence as forming concepts and categories? If the answer is positive, then how and why does metonymy function in the early stage of cognition? And is there any empirical evidence? In addition, since all the interpretations of "contiguity" are related to a WHOLE such as a domain/frame/ICM, how is this kind of WHOLE category formed?

These questions will be answered in the following chapter. There it is argued that metonymy is a natural cognitive mechanism rather than merely a cognitive process. This mechanism operates perceptually as well as conceptually. In terms of development, it consists of two stages in cognition. In the first stage, it picks up some prominent element(s) in an interactive process to form a patterned experience; once such a pattern is formed, it then moves to the second stage, where PART-FORWHOLE, WHOLE-FOR-PART and PART-FOR-PART RELATINGs take place. That is, the patterned experience enables a part to mean another part, and a part to mean the whole or vice versa. What current research on metonymy argues about is all in the second stage of cognition, e.g. PART-FOR-WHOLE, WHOLE-FORPART (and PART FOR PART). That is also why the cognitive views of metonymy all place emphasis on a whole frame/domain/ICM; it has to take the first pattern forming stage as its prerequisite. Here, "natural cognitive mechanism" means metonymy is not an ability to be learned or acquired; instead, it means metonymy functions since birth and beyond the conscious awareness. "Pick up" indicates subjectivity in embodied cognition, with the meaning that attention is only paid to something interesting or something attracting, whether it is something in focus or something as a whole in the range of what is perceived or conceived. "A patterned experience" means a discrete experience which forms a sort of formula or logical 
pattern in cognition, and which is generally reflected by or embodied in patterned neuron networks. ${ }^{24}$

Chapter 3 is devoted to the illustration of this definition of metonymy as a natural cognitive mechanism in terms of concept formation, conceptual development, categorization and memory, from the perspective of developmental psychology, cognitive psychology, and neuroscience in the framework of Embodied Cognition Theory.

\subsection{Summary}

This chapter examined some cognitive approaches to metonymy. The reference point/mental access view considers metonymy as a reference point phenomenon in which the source provides mental access to the target because of cognitive salience. However, the cognitive salience principle is not omnipotent and does not apply everywhere and every time; it involves subjectivity and pragmatic function. In addition, in some cases, metaphor might be viewed as a reference point phenomenon as well, for in conventionalized metaphor, the source also provides mental access to the target via a subjective perceived similarity relation between the source and the target whether in shape, size, appearance, feature, or function, due to the conventionalization.

The contingent indexical approach sees metonymy as an indexical relation and regards the target meaning in metonymy as a type of meaning elaboration. Yet as pointed by Warren (1998), metonymy can also be based on an iconic relation.

The blending theory attempts to explain metonymy and views it as a blending phenomenon involving integrating at least two input spaces into a blended space with the help of a generic space. Nevertheless, the elements selected from the space to speak for it before blending have to be motivated by metonymy per se, i.e. it is because metonymy operates here that the particular part can be picked up from the input spaces to blend and yield an emergent structure space. Thus when the blending theory explains a metonymic phenomenon, it needs metonymy to support the blending. The blending theory therefore is pointless when it comes to metonymy.

\footnotetext{
${ }^{24}$ In this sense, it is similar to ICM or Frame.
} 
While the cognitive domain view seems to make a distinction between metonymy and metaphor, it involves some definitional problems and boundary problems in "domain" and "mapping". Besides, its use of one and the same term/mechanism (i.e. mapping) to try to tell metonymy from metaphor suggests controversy, confusion, and unsuccessfulness. This implies that the long-held view of the contiguity relation in metonymy is not ultimately refreshed.

If viewed from the standpoint of Embodied Cognition, especially from neuroscience, the contiguity relation can be replaced by a patterned neural network, or in other words, patterned experience. Furthermore, those cognitive approaches to metonymy unanimously view metonymy as a cognitive process or model, and they seem to have ignored the force behind the process/model. That is, when metonymy is considered as a process or model, what exactly makes this process or model feasible needs to be known. And what is more, how the WHOLE is formed before the PART is permitted to speak for the whole or vice versa, and to speak for another part, has to be determined. 


\section{Chapter 3 Metonymy as an Innate Cognitive Mechanism}

The previous chapter examined current cognitive views of metonymy and their interpretations of the "contiguity" relation in metonymy, followed by a tentative claim of metonymy as an inherent cognitive mechanism. It could be that the cognitive research on metonymy is largely based on linguistic (both language and sign language) data, as well as on pictorial and multimedia, gestural and sign data (Kwiatkowska, 2007; Mujic, 2009; Pastra, 2009; P. Wilcox, 2004), and metonymy is found to be conceptually present; that is why metonymy is also termed conceptual metonymy. In other words, the current cognitive research on metonymy, for the most part, is devoted to language/sign-involved conceptual activities.

Summarily, metonymy in this perspective is regarded as a cognitive process in which the source, acting as a reference point, provides mental access to the target in the same domain/frame/ICM, with PART-WHOLE as the prototypical relation, i.e. metonymy is an intra-domain mapping between PART and WHOLE. This viewpoint, however, also raises some questions. For example, metonymy is viewed as a cognitive process, but is this process self-propelled or driven by other cognitive elements? Metonymy is considered to function by relating PART and WHOLE within a whole (such as the same domain/frame/ICM), but how is the whole formed before it enables the PART-WHOLE relating, does metonymy operate in forming the whole as well? It seems that so far the research on metonymy is confined to the conceptual activities mainly involving language(sign, gesture), but does metonymy operate in cognitive activities beyond language involvement, e.g. does metonymy function in cases without language influence or with language influence least felt, i.e., in infants and subjects with cognitive impairment? This chapter contributes to answering these questions.

In this chapter, it is tentatively claimed that metonymy is instead an innate cognitive mechanism involving two processes. It first enables elements to form a patterned experience, which will further license PART-FOR-WHOLE, PART-FOR-PART, and WHOLE-FOR-PART cognition. That suggests that, as a natural cognitive mechanism, metonymy does not merely operate within a certain frame/domain as argued in current cognitive research on metonymy, it also functions in the process of 
forming the whole pattern. Metonymy as an inherent cognitive mechanism operates perceptually as well as conceptually and operates in infancy when language influence is least felt and in subjects with impaired language ability. This chapter will first of all consider the features of metonymy obtained in the cognitive research on metonymy so far, and then interpret how metonymy operates in perceptual and conceptual categorization, category and concept development, inductive inference (generalization), and memory in light of cognitive science scholarship, with focus given to these cognitive activities in infants and brain-impaired subjects from research experiments in cognitive psychology, developmental psychology, and neuroscience.

\subsection{Features of metonymy}

As mentioned in the preceding chapter, it might be said that there are four main approaches to metonymy from the cognitive perspective. Although there are some differences among these views, consensus on the essential characteristics of metonymy has been reached, as listed below:

1) Metonymy is a cognitive process motivating language structures and language uses. For example, grammar is metonymic in nature (Langacker, 2004), see also Panther (1994, 2005), Panther \& Radden (1999), Panther \& Thornburg (2003b), Panther et al. (2009).

2) Metonymy takes place within a whole such as a frame/domain/ICM. For example, in the widely held domain view, the various definitions and contiguity interpretations mentioned in the previous chapter.

3) The prototypical relation of metonymy is PART-WHOLE. This prototype can be embedded dimensionally, e.g. from spatial to temporal to categorical domains, from bounded to unbounded, from physical contact to adjacent without contact (Peirsman \& Geeraerts, 2006).

4) Metonymy is ubiquitous in language and more elementary than metaphor. For instance, Radden (2005), Barcelona (2000c, 2002b), Mittelberg \& Waugh $(2008 ; 2009)$.

These features are consistent with the previous chapter's claims of what counts as metonymy, relisted below: 
$\mathrm{A}$ and $\mathrm{B}$ are within a WHOLE (i.e. in the same ICM/Frame/Domain)

$\mathrm{A}$ and $\mathrm{B}$ are functionally linked in experience

A activates B (B is mentally accessed while A is mentioned/projected)

The claims of metonymy as a cognitive process/model just like metaphor and as ubiquitous in language are based on the extensive cognitive analysis of linguistic structures and uses, such as the various levels of the language system (e.g. phonetic, morphological, syntactical, lexical aspects), metonymy-motivated language structures (Ruiz de Mendoza \& Pérez Hernández, 2001), language phenomena in use, e.g. making pragmatic inferences, speech act scenarios, meaning construction, conversion of word classes (Dirven, 1999). This is the area most Cognitive Linguistic study of metonymy lies in. ${ }^{25}$ This linguistics analysis of metonymy finds that the various relations in metonymy are viable only in a certain range or pattern, for instance,

(20) Does he have wheels? (Aitchison, 2003)

In this metonymy of "wheels for car", i.e. PART-FOR-WHOLE, it is only plausible when the car has wheels, i.e. the car having wheels as a whole enables the wheelsfor-car metonymy.

However, as listed in Chapter 2, there exist a large amount of relations in the category of metonymy such as CAUSE-FOR-EFFECT, PRODUCT-FORPRODUCER, and LOCATION-FOR-LOCATED, and it is impossible to exhaust the relationships possibly existing in the category of metonymy. Metonymy as a category therefore has prototypes and the prototypical relation in metonymy is accordingly PART-WHOLE (Peirsman \& Geeraerts, 2006). All other relations can be interpreted in terms of the PART-WHOLE relation (Radden \& Kövecses, 1999; Ruiz de Mendoza, 2000; Ruiz de Mendoza \& Díez Velasco, 2002). This PARTWHOLE prototype in metonymy develops multi-dimensionally, starting from spatial PART-WHOLE containment over physical contact to adjacency without contact in terms of strength of contact, towards unbounded wholes and parts in terms of

\footnotetext{
${ }^{25}$ One point should be made clear here, that is, metonymy in Cognitive Linguistics or in the cognitive perspective is very general, as the analysis in Chapters $1 \& 2$ demonstrated. The traditional rhetoric figures of speech such as euphemism, hyponymy, synecdoche all fall into the prototypical category of metonymy.
} 
boundedness, and towards temporal, spatio-temporal and categorical domains in terms of cognitive domain (Peirsman \& Geeraerts, 2006). In brief, the prototypical PART-WHOLE advances from the concrete to the abstract, which is in line with the view of Embodied Cognition that concept and conceptual processing are grounded on perceptual sensorimotor experience. Nevertheless, the prototypical presentation of metonymy does not tell how the PART-WHOLE relation is formed, even though it correctly describes the development of this relation within the category of metonymy.

More examples are given below to have a clear idea of these features of metonymy.

(21) A: We need another pair of hands, but Adam couldn't bring himself to replace Mike. They were together so long.

B: How long were you with him?

A: Eight years. I am an attachment from Number 10.

B: Oh, poor Number 10. (Movie: Ghost Writer)

(22) Please look out for the pedestrians whenever you are behind the wheel. (New Zealand Road Code)

(23) We'll be right there. Besides, it's good to have somebody around here that pees standing up. (Movie: The Devil's Own)

(24) Your 10:00 is here, the hospital discharged him this morning.

In example (21), "pair of hands" metonymically means a person with emphasis on hand work, e.g. "(ghost)writing" autobiography for Adam the Prime Minister in here; "an attachment" is metonymical for an aid/assistant; the first "Number 10" is metonymical for the UK Prime Minister's Office at 10 Downing Street, i.e. STREET NUMBER FOR RESIDENCE; the second "Number 10" is a further metonymy for the Prime Minister, involving a chain metonymy, i.e. STREET NUMBER FOR RESIDENCE FOR RESIDENT. Here, hands are part of a person as a whole; that an attachment is someone attached to some place (as a whole), which illustrates a general-specific relation, can also be regarded as a PART-WHOLE relation 
according to the prototypical view of metonymy. One of the two Number 10s is the street number for the place/building, and the other is street number for the person (the Prime Minister) working there (within the Frame/ICM of UK Prime Minister, i.e. the experienced pattern of knowledge about UK Prime Minister), which can both be considered as a PART-WHOLE relation since they are all in the same Frame/ICM.

In (22), "behind the wheel" is a metonymy for driving. The action of driving consists of getting into the car, sitting behind the wheel, pushing the pedals, etc., thus "behind the wheel" is part of driving as a whole.

In (23), "somebody around here that pees standing up" is metonymical for a man. "Pees standing up" is one of the typical properties of a man, i.e. a typical feature of man stands for man, hence a PART-WHOLE relation according to the prototypical view of metonymy held in this research.

In (24), "10:00" is a metonymy for the person with whom you have an appointment at ten o'clock. Actually this example, like the second "Number 10" in example (21), consists of a chain metonymy (See Chapter 7 for details of chain metonymies), i.e. "10:00" is first metonymical for the appointment at 10:00, which is then metonymical for the person for whom the appointment is arranged. Therefore PARTWHOLE relations are involved.

Nevertheless, from these features of metonymy in the cognitive perspective some questions arise. For example:

1) Is metonymy merely a cognitive process? If it is a cognitive process, what empowers its operation?

2) While the wholeness is emphasized and the PART-WHOLE relating within the whole is focused on, how the whole is formed is neglected. Does metonymy operate in the process of forming the whole as well or just operate as a cognitive process relating PART and WHOLE after the whole is formed?

3) Does metonymy operate in cognitive activities without language or with language influence least felt? 
In this research it is tentatively claimed that

1. Metonymy is an innate cognitive mechanism, which operates naturally and unconsciously.

2. Cognitively metonymy first operates to form a whole pattern (e.g. category, concept), i.e. PART-WHOLE FORMING, and then relates PART and WHOLE within the patterned experience, i.e. PARTWHOLE RELATING; besides, it also relates PART and PART within the whole pattern, e.g. from spatial to temporal in an event/action scenario $^{26}$.

3. Metonymy operates both perceptually and conceptually, i.e. it functions beyond language as well as with language. Both languageinvolved and language-beyond cognitive activities are underpinned by metonymic operation.

In order to justify this claim, this chapter will argue for the PART-WHOLE FORMING and PART-WHOLE RELATING roles of metonymy through examining perceptual and conceptual categorization, category and concept development, inductive inference, and memory organization and development in infancy when language does not take effect, in light of cognitive science research.

\subsection{Metonymy as an inherent cognitive mechanism}

\subsubsection{The WHOLE Prototype in Embodied Cognition}

The reasons why metonymy operates in the early stage of cognition are mainly from the research in cognitive science and neuroscience. In accord with the feature of metonymy, i.e. metonymy takes place within a WHOLE, what about the WHOLE in the early stage of cognition? If there is a WHOLE, how is it formed? Cognitive science research suggests that human beings were born with a WHOLE, i.e. the body schema, which influences cognition and determines cognition to go from the body to the surroundings and from self to others. This primitive WHOLE may be evidenced

\footnotetext{
${ }^{26}$ Although "frame" and "ICM" seems similar with "scenario", in my view, an embodied cognition account of metonymy should favour "scenario", because "scenario", by comparison, is much closer to the idea of embodied (perceptual and sensorimotor) experience and "frame" and "ICM" may sound more static and abstract. Besides, scenario is closely related to action (event), which is considered as the unit of, and thus crucial in, embodied cognition in cognitive science.
} 
by, at the most basic level, the body schema, embodied cognition, and neural networks, to list just a few.

Body schema: Research in cognitive science and neuroscience suggests that human body plays a crucial role in cognition, and in fact the body shapes human thinking (e.g. Boroditsky \& Ramscar, 2002; Chiel \& Beer, 1997; Gallagher, 2005; Johnson, 1987; Varela et al., 1991; Ziemke et al., 2007). Human beings were born with a body schema, which is the mental construct of the ideal body, and which is evidenced to operate under human thinking and behaviour (Gallagher, 2005). Phantom sensations reported by children who were born without one or more limbs suggests that the brain might be genetically predisposed to represent a prototypical human body, regardless of the correspondence or lack thereof between ideal model and actual body, and this corporeal awareness is evidenced at a neural level (Berlucchi \& Aglioti, 1997). Therefore, it seems that human beings were born with a prototype WHOLE already there, i.e. the body schema, which implies the likelihood of the WHOLE providing mental access to the PARTS and vice versa, e.g. body and body parts.

Embodied cognition: As pointed out early in Chapter 1, research in cognitive science and neuroscience also suggests that conceptual activities are grounded on the sensorimotor experience with the physical and socio-cultural environment, which is eventually represented in the form of neural substrates and networks (e.g. Barsalou, 1999b; Feldman \& Narayanan, 2004; Gallese \& Lakoff, 2005; Hauk et al., 2004; Lakoff, 2009).

Neural networks: In the research on the neural basis of cognitive activities, e.g. action verbs (Hauk et al., 2004; Willems et al., 2010), image schemas (Dodge \& Lakoff, 2005), abstract concepts (Gallese \& Lakoff, 2005; Kosslyn et al., 2001), sign language processing (Corina et al., 1996; Knapp \& Corina, 2010), and interplay of gestures, language and actions (Willems \& Hagoort, 2007), neurons are found to function in groups, which are meaningful and enter into neural computation as nodes to form neural networks (Lakoff, 2009). In other words, the brain, though born with a body schema, is neurally wired and consists of comparatively independent but interacting neural networks, in which the firing of some neurons will activate the whole neural network they operate in and are related to. This can be considered as 
the neural basis of PART-WHOLE and PART-PART metonymic processes. For example, Cortical premotor areas contain neurons that respond to multimodal (visual, somato-sensory, and auditory) sensory stimuli, and posterior parietal areas play a major role in motor control, but these areas are neurally integrated not only to control action, but also to serve the function of constructing an integrated representation of (1) actions together with (2) objects acted on and (3) locations toward which actions are directed (Feldman \& Narayanan, 2004).

Since human beings have this body schema as a WHOLE in the brain, under its influence and also due to the limitation of the body (e.g. it is not possible to intake and memorize too much a time), they are predisposed to assume that there is a mental WHOLE somewhere with indeterminate boundaries that may need to be proactively specified and brought out in embodied experience. This might be the reason humans are born to categorize (Mandler, 1997; Quinn, 2011). This perhaps psychologically allows for concept and category formation without conscious awareness.

This category might be very rough and hard to define initially. Repetition of exposure to the same object would normally subserve and facilitate the emergence of the WHOLE (i.e. category), but comparison after exposure to other objects would clarify the WHOLE.

Thus, in the formation of concepts and categories, human beings are still performing the PART-WHOLE process --- they are specifically establishing and updating the WHOLE under the influence of the mental WHOLE prototype. They are clarifying the WHOLE in the PART-WHOLE forming way. That is why the first object concepts tend to be global in nature and gradually become differentiated with experience (Mandler, 2007a). In this sense, Domain/Frame/ICM, which is considered as the WHOLE in Cognitive Linguistics, is itself metonymically formed.

If this is the case, then it might be said that metonymy operates since birth and is therefore a natural cognitive mechanism, which does not need learning and acquisition, and does not need other cognitive factors to drive its operation. 


\subsubsection{Metonymy in categorization: From PART to WHOLE}

The importance of categorization in cognition cannot be exaggerated. It is viewed as an inherent basic cognitive ability by most scholars across disciplines in cognitive science. For example, from communication studies, Trenholm \& Jensen (2004, p. 140) point out that humans have an insatiable appetite for categorization; from Cognitive Linguistics, Lakoff (1987b, p. 8) argues that the capacity to categorize is the most fundamental of human capacities; from developmental psychology, Mandler (1983, p. 466; 1994, p. 48) claims that categorizing itself is a basic ability and certainly must be posited as a primitive of psychological functioning, claiming "categorization is an essential aspect of all perceptual systems; it is what enables implicit recognition of old stimuli seen in new aspects and of new stimuli that are similar to those experienced in the past", and "it is actually a rather primitive function, found in all organisms, including the very young of our own species." Also in developmental psychology, Thelen \& Smith (1994; see Quinn, 2011, p. 129) describe categorization as the primitive in all behaviour and mental functioning; Quinn (2011) asserts that humans are born to categorize and cognition is founded on categorization. From neuroscience, Edelman (1987, p. 7, see Quinn, 2011, p. 129) argues that "one of the fundamental tasks of the nervous system is to carry out perceptual categorization in an unlabeled world". Categorization therefore is a natural elementary cognitive ability.

Although there is some debate as regards the necessity of distinguishing perceptual categorization from conceptual categorization, see e.g. Rakison \& Oakes (2003), following Mandler (1996, 2000, 2003, 2004a), this research treats perceptual categorization and conceptual categorization as different from each other. However, it also follows the common belief in Embodied Cognition research that concepts are grounded on perceptual and sensorimotor activities, and knowledge about the world originates from perception of the world. Thus conceptual categorization is also derived from perceptual observation of the world. Despite the perceptual and conceptual categorization dispute, it is likely that the mechanism underlying these two categorizations is the same, at least metonymy in general, and the PARTWHOLE FORMING process in particular, plays a crucial role in categorization. 
According to Mandler (1996, 2000, 2003, 2004), perceptual categorization is based on perception of salient perceptual features, i.e. on what an entity looks like; it functions to enable object identification and recognition. But the salient perceptual features have to be focused in perception, which is determined by the physiological characteristics of man. Embodied Cognition claims that the physiological characteristics, together with the goal, determine what can come to the perception and what can be perceived, which, even cross-modally, is only part of the situation at the time of perception; only a small part of what is perceived can be focused on and attended to. For example, the eyes can only look to the front and must simultaneously disregard the back; even within the sight of the eyes, only a specific part can come to the perception. Thus the fate for much of the perceptual information in process is probably "Looking without seeing" 27 and "inattentional blindness" (Mandler, 2004a, p. 69).

A large amount of psychological research on infant categorization, see e.g. Rakison and Yermolayeva (2010), illustrates that in the perceptual categorization of objects the shape or appearance of the object is often attended to. Infants differentiate objects according to their parts. For example, they differentiate animals from vehicles through perceiving legs versus wheels (Rakison, 2003; Rakison \& Butterworth, 1998; Rakison \& Cohen, 1999). Newborns are able to form broad categories of distinguishable geometric shapes by relying on the shapes' perceptual similarity — closed versus open geometric forms (Turati et al., 2003).

In the perceptual categorization of nonhuman animals, research shows the head of animals are frequently attended to. For example, in familiarization and preference tests using an eye tracking technique, 6- to 7- month-old infants tend to form a perceptual category of animals (e.g. cat versus dog) relying on the head rather than on the body; their eye fixation is more on heads than on bodies, even with a cat head on a dog body versus a dog head on a cat body (Quinn et al., 2009; Quinn \& Eimas, 1996). In perceptual categorization of humans, even 3- to 4- month-old infants can distinguish male from female by attending to the face, i.e. they tell male from female on the internal facial features in their upright orientation, not on hair cue or other

\footnotetext{
${ }^{27}$ As early as six hundred years ago, Leonardo Da Vinci said "People look without seeing, listen without hearing, touch without feeling, eat without tasting, move without physical awareness, inhale without awareness of odour or fragrance, and talk without thinking." See Gelb (1998, p. 97).
} 
features (Quinn et al., 2002). Infants aged 5- to 6- months attend to the features of a face which define its age or sex in face recognition (Fagan Iii \& Twarog Singer, 1979). But when dynamic events are involved, it is the salience of action, not faces any more, that matters in infants' perceptual categorization of humans (Bahrick et al., 2002). Some developmental psychology experiments suggest that perceptual categorization of the human body is different from that of other objects. The perceptual categorization of animate objects is more based on their visual characteristics or parts, but the perceptual categorization of inanimate objects is more based on their functional characteristics or parts (Rakison \& Butterworth, 1998; Reed et al., 2004). As for perception of motion events, correlation among member parts is focused on; even infants of 10-18 months can attend to some correlations among perceptual features such as correlations among parts, body, motion path (Rakison, 2004; Rakison \& Poulin-Dubois, 2002). Infants seem to naturally attend to a particular part or section to form distinguishable perceptual categories of inanimate objects, humans and other animates, and even motion events.

However, actions are evidenced in psychological experiments to be the crucial part in conceptual categorization, which is based on mental interpretation of perceptions of (inter)actions. While perceptual categorization is about what an entity looks like, conceptual categorization is about what an entity is, i.e. it forms "kind knowledge" (i.e. what kind of entity it is or belongs to, or in other words, category knowledge) of the entity from its (inter)action with surroundings. Thus conceptual categories are formed as a whole from actions as the salient aspect of the perceived entity, i.e. it is a kind of action feature forming the whole. Although perceptual features might and will eventually be embedded in the concept of the entity, in forming the concept of what the entity is, perceptual features or similarities are rather inferior (see e.g., Mandler, 2004a). Infants of 7- to 11 months did distinguish airplanes from birds even though they perceptually look alike with a similar shape, and they did not differentiate dogs from fish or from rabbits even though they look quite different (Mandler \& McDonough, 1993). Action organizes infants' conceptual world (Mandler, 2006; Pecher \& Zwaan, 2005) and influences perceptual and conceptual development (Rakison \& Woodward, 2008). Action relates perception, conception and language as well. Even neural patterns are centred on embodied actions (see e.g. Willems \& Hagoort, 2007). Concepts do not arise from perceptions directly, they are 
results of perceptual meaning analysis, which relates what is perceived to the perceiver in the environment and interprets it in terms of its meaning to the perceiver in the form of image schemas; thus at the very beginning, owing to their rudimentary foveal state, infants will form very general or global concepts, which will be enriched and detailed with more experience and sensory development (Mandler, 1988, 1992, 1994, 1997, 1999, 2003, 2004a, 2005, 2007b, 2008). For example, observing dog/cat/fish moving and trolley/table/chair being pushed, infants from the age of 7 months form global conceptual categories of self-motion and caused-motion (Mandler, 2000; Mandler \& McDonough, 1993, 2000).

Action is crucial to conceptual categorization, and it also influences the formation of some perceptual categories. ${ }^{28}$ For example, action even influences shape categories (Smith, 2005). The way an object moves an object changes shape categorization and helps to make inferences about the shape and material of objects; for instance, observing a sponge being squeezed or a steel ball being rolled, infants form different categories of shape and material, i.e. a sponge can be squeezed, it is soft material with changeable shape; a steel ball can be rolled, it is hard material with stable shape (Kobayashi, 1997). Research shows that even at the age of 5.5 months, infants are more likely to process and remember information about what people around them are doing than what they or the objects being used look like (Bahrick et al., 2002). The conceptualization of human body is based on how it performs actions, and the conceptualization of other objects appears to be shaped by how the human body interacts, or doesn't interact, with the objects (Reed et al., 2004). Experiments show that 11- to 12- month-old infants use functional information (i.e. what an object or part of it can do or what can be done to it) as a cue to conceptual categorization despite the overall similarity (Träuble \& Pauen, 2007). Other psychological experiments also demonstrate that young infants are sensitive to the difference between caused motion and self-motion (Leslie, 1988; Poulin-Dubois et al., 1996), as Mandler and her colleague showed; between contingent and non-contingent interaction among objects (Frye et al., 1983); between biological and non-biological motion (Arterberry \& Bornstein, 2001).

\footnotetext{
${ }^{28}$ It should be pointed out here that it is not action proper that shapes the perception of an object, but instead it is the conceptual categories derived from the action that exerts impact on the formation of the perceptual categories.
} 
In sum, both perceptual and conceptual categories in infants are essentially formed through picking up a salient part or section of an entity such as shape, appearance, face, motion, and action as the perceived to form a general or global category as a whole. According to the view of metonymy, it might be said that it is basically metonymy here that licenses the mental PART-WHOLE FORMING in categorization. Given that categorization is an inherent cognitive ability, metonymy, as the drive and underlying mechanism of categorization, is highly likely to be a natural cognitive mechanism.

\subsubsection{Metonymy in category and concept development: From WHOLE to PART}

Since Rosch and her colleague's research on category (Rosch, 1973; Rosch \& Lloyd, 1978), it has long been held that the basic level category is the first to form and acquire, and then based on the basic level category, super-ordinate and sub-ordinate categories are derived. That is, the general view of category development is from the basic level to super-ordinate level and sub-ordinate level. However, recent research in developmental psychology suggests that the basic level category may not be the first category formed in infancy, and in fact the basic level category is influenced by human labelling. The first category formed in infants is instead global, i.e. rather general, not really the so-called basic level category. Even if it is very general and global, this first category cannot be termed as super-ordinate, because there seem to be no subordinate parts at all in infancy when they form conceptual categories; they just form a general category out of their particular focus, no hierarchy at all initially. The particular focus they take advantage of to form a category would be ignored once the category is formed. Thus the first formed category or concept is rather general and global, very rough in the sense that they don't have enriched knowledge about it and are not aware of the category knowledge. Accordingly category and concept develop from global/general to local/specific or from broader to narrower (Behl-Chadha, 1996; Mandler, 1989, 1997, 1999, 2004a; Mandler \& Bauer, 1988; Mandler \& McDonough, 1993, 2000; Quinn \& Eimas, 1998; Quinn \& Johnson, 2000; Younger \& Fearing, 2000). Initial categories are evidenced to be very general and they become enriched and gradually specific and narrower with experience and cognitive development. Research shows that 2- and 4- month-old infants, familiarized with instances from a number of mammal categories, e.g. cats, dogs, 
tigers, rabbits, zebras, elephants, form a global representation of mammals that includes novel mammal categories but excludes instances of non-mammalian animals such as birds and fish and human-made artefacts, e.g. furniture (BehlChadha, 1996; Quinn \& Johnson, 2000). Infants of 7- month old form global conceptual categories of animals, vehicles, and furniture but they did not differentiate between dogs and fish (i.e. members of animal category), between cars and airplanes (members of vehicle category) until 18 months; they did not consistently differentiate between dogs and horses, cars and trucks until 30 months (Mandler, 1997; Mandler et al., 1991; Mandler \& McDonough, 1993). Research evidence shows that 16- and 20- month-olds differentiate basic-level categories only when the categorical contrasts are taken from different super-ordinate or global classes, e.g. they differentiate dogs from cars, but they did not differentiate the categories drawn from the same super-ordinate class, e.g. they don't differentiate dogs from horses (Mandler \& Bauer, 1988).

Categories and concepts are formed as a global or rather general whole, which is consistent with infants' biological, physiological and cognitive development, for in the early period of life, infants are in a rudimentary state in sensory perception and motor action. With their development in these aspects and their embodied experience in the physical and socio-cultural environment, the initially formed general categories and concepts will be enriched and take in more and more members, thus finally forming a hierarchy of membership. During this global to specific development, it might be mainly metonymy that enables the occurrence, i.e. it goes from WHOLE to PART. It is the WHOLE (e.g. category or concept), i.e. the kind knowledge the infants develop ${ }^{29}$, that validates encapsulating PARTS. These take place mentally without conscious awareness. While category and concept formation is essentially PART-WHOLE FORMING by metonymy, category and concept development is basically PART-WHOLE/PART RELATING ${ }^{30}$. Once the WHOLE is formed from PARTS, the WHOLE will take charge in inductive generalization

\footnotetext{
${ }^{29}$ According to Mandler (1997, 2004), both (conceptual) category and concept are summary kind knowledge; conceptual category stresses the extension of a concept, whereas concept emphasises the intension of a notion; perceptual categories, or rather perceptual schemas, are summary representations of what things look like without any intension.

${ }^{30}$ For the sake of convenience, the term of PART-WHOLE/PART RELATING covers the situations of WHOLE TO PART, PART TO WHOLE, and PART TO PART.
} 
and memory as well as in category and concept development, and also will enables PART-PART RELATING.

\subsubsection{Metonymy in inductive inference: From WHOLE to PART}

Inductive inference /generalization experiments on infants suggest that infants use the kind knowledge of category and concept they have acquired through PARTWHOLE FORMING to make inference/generalization, which is guided by the WHOLE (i.e. the kind knowledge of what an entity is). They also thereby predict and expect what will happen or what is next. Inductive inference fundamentally goes from the WHOLE to the PART. The kind knowledge of a category and/or a concept controls infants' inference/generalization. Generalized imitations can tell how infants make inference/generalization. For example, 9-to14- month-old infants were shown someone giving a dog a drink from a cup, going to sleep in a bed, keying a car, and a car giving a person a ride, and then the infants were given different animals and vehicles along with the relevant prop (e.g. the cup, the key) to imitate what had been modelled. They were found to give a drink to cats, rabbits, fish, birds, anteaters, and armadillos, and put them in bed, but did not extend this to vehicles; and found to key trucks, buses, motorcycles, airplanes, forklifts, and shovelers and give rides in them, but not animals. After being given a demonstration of someone giving both a dog and a car a drink or keying both a dog and a car, they typically did not use the inappropriate object, e.g. they did not key any of the animal models or give a drink to any vehicle models given (Mandler \& McDonough, 1996, 1998; McDonough \& Mandler, 1998). The infants made the right inductive inferences widely within the animal and vehicle categories respectively, although the props from different categories were mixed together in the experiments. Their generalization seems to be guided by the kind knowledge, i.e. the category or concept as a whole. Infants' inductive inference seems to go from the whole to the part, from the general to the specific. This metonymic development may be evidenced in collision, occlusion, and containment event experiments as well.

In a collision event consisting of a moving and a stationery object, 5.5-6.5 montholds engage in calibration-based reasoning, i.e. they reason on the proportional relation between the size of the moving object and the distance travelled by the stationery object (Kotovsky \& Baillargeon, 1994, 1998). Other experiments 
involving collision events also suggest that 9-month-olds reason a box as movable or immovable on the width of the box; they infer that the wide and tall-square box as being immovable and the tall-narrow box as movable and expect the wide and tallsquare box to remain stationary and the tall-narrow box to be displaced, and were found to be quite surprised when the expectation was violated (Wang et al., 2003).

Occlusion event experiments indicate that even 2.5 month-olds expected objects to become visible when passing between occluders and to remain hidden when passing behind occluders (Aguiar \& Baillargeon, 1999, 2002). They categorized the events as occlusion and reasoned about the object individuation on occlusion (i.e. the particularities of the objects involved) whether the occluders were opaque or transparent (Wilcox \& Chapa, 2002).

Even without habituation and familiarization trials, 4-month-old infants still could represent and reason about hidden objects, and they were surprised when a wide object became fully hidden behind a narrow occluder or inside a narrow container (Wang et al., 2004).

While in containment events (e.g. an object was lowered inside a container with either a wide opening or no opening in its top surface; an object was lowered either behind or inside a container and the container was moved forward and to the side, revealing the object behind it), 3.5-month-old infants in the experiments looked reliably longer at the closed- than at the open-container event, and at the inside- than at the behind-container event. These suggested that the infants recognized that the object could be lowered inside the container with the open but not the closed top, and that the object could not pass through the back wall of the container and hence should have moved with it to its new location (Hespos \& Baillargeon, 2001).

In the experiments of collections (i.e. a collection of objects could be conceived of as individual objects or as an individual collection, e.g. sand), 8-month-olds were surprised when a single object violated the continuity principle (i.e. the single object does not follow the kind knowledge of the category); however, they were not surprised when a collection of objects did so (Chiang \& Wynn, 2000). 
Even new-borns of just a few hours old could categorize sight-sound pairings and infer them relying on the sound as an attribute of a specific object even though the objects were in different locations (Morrongiello et al., 1998).

All these experiments demonstrate that infants, even before they can speak, make inductive inference relying on the kind knowledge they formed through PARTWHOLE FORMING in their perceptual and conceptual activities. Once they formed the whole, e.g. category or concept, they will accordingly generalize and infer, i.e. they will fundamentally undertake PART-WHOLE RELATING conceptual activities. It could be that the part(s), e.g. objects and props in the experiments, would activate the WHOLE (the kind knowledge of the category or concept) and then the WHOLE would take charge for the inference and generalization. The inference and generalization is essentially from WHOLE to PART.

\subsubsection{Metonymy in memory: PART-WHOLE FORMING and RELATING}

Memory comes out of PART-WHOLE FORMING, but its organization and development is actually PART-WHOLE RELATING. Entities (objects, events, etc.) do not come to the mental world in full form, but in the form of a salient part of it, i.e. only the salient part of an entity, taken as the entity, comes to the mind for later identification, recognition, and recall. This is a process of PART-WHOLE FORMING, enabled by metonymy, as argued in the previous section. Once the whole is mentally formed, memory takes place on the whole but relating to the part memory is based on. Memory is centred on the salient part which forms a whole, and the whole then controls memory with the salient part such as objects or object related actions as the trigger. Memory thus involves the PART-WHOLE RELATING process as well. That is to say, it is principally metonymy that empowers the initiation, organization, function and development of memory, even in infants.

To see how infants' memory develops and works, deferred imitations are often used, i.e. an event is modelled while infants watch, and after a delay they are given the objects used in the modelling and encouraged to imitate what they observed. Research experiments show that at 9 months infants can reproduce temporally ordered events after delays of a month; by 10 to 11 months, infants can reproduce 
temporally ordered events after delays of 3 months (Carver \& Bauer, 2001; Mandler $\&$ McDonough, 1995). At the age of 9 months, infants can recall two-action causal events after 24 hours, but cannot recall two-action arbitrary events even after a 20-s delay. After a brief period of observational learning of two-action novel causal events, 11 month-olds can reproduce many of the events in their entirety in the correct order both after 24 hours and a 3-month delay, even though they often remember only one of the two actions. These research results suggest that in order to imitate the modelling after a delay, the infants have to form a logical whole event mentally from parts of their observation and then accordingly trigger the whole event when they are shown the prop used in the modelling after a delay. Two-action arbitrary events could not be imitated, because they were not mentally taken as a whole when they observed the modelling. Novel events with causal relations can be recalled, even with interruptions in the experimental manipulation (Bauer \& Mandler, 1989, 1992). As mentioned in Section 3.1, above, infants attend to the relations existing between actions. This allows them to keep them as a whole in their memory and to recall for imitation after a delay.

Just like concept and category, infants' memory keeps the initially formed whole, which lasts even after all detailed information is lost. Global categories are more resilient and longer-lasting. Memory is organized in a hierarchical way just like the conceptual formation, i.e. from global/general to specific. The data on acquisition and breakdown of the semantic system suggest that super-ordinate categories are more resilient and longer-lasting than more detailed subdivisions, and the superordinate (i.e. the global) ${ }^{31}$ is the "first in, last out", because more detailed concepts are constructed out of super-ordinate ones and their most fundamental meaning stems from their super-ordinate class membership (Mandler, 1998b). Semantic dementia research also shows that memory breakdown goes from the loss of specific details to more general categories (Hodge et al., 1995); meaning of the class/category as a whole determines what gets associated with what (Mandler, 1998). In Hodge et al (1995), the patient first lost discrimination between specific category instances, e.g.

\footnotetext{
${ }^{31}$ Although the first category cannot be termed as super-ordinate due to short of members as pointed out earlier, after embodied experience and cognitive development, this global category will be enriched and become hierarchical, thus becomes super-ordinate. It is in this sense that "super-ordinate" is used here.
} 
dogs vs. horses or cars vs. buses, then told that an object was a flying animal, or a tool, or something to sit on, the last distinction was the animate-inanimate.

All this seems to support the idea that metonymy operates in memory organization and development, which could be best demonstrated by memory breakdown studies. It is likely that metonymy organizes memory through the WHOLE which is metonymically formed from part of the actions or events, and is in charge of memory development and reconstruction by recursively relating PART and WHOLE.

\subsection{Spatial to temporal: PART-PART relating}

Since metaphor is viewed as a cross-domain mapping while metonymy as an intradomain mapping, in cognitive science in general (Boroditsky, 2000, 2002; Casasanto \& Boroditsky, 2008; Casasanto et al., 2010; Gentner et al., 2002; Mandler, 2010; Torralbo et al., 2006), and Cognitive Linguistics in particular (Gentner \& GoldinMeadow, 2003; Gibbs, 1994; Johnson, 1987, 2007; Lakoff, 1987b; Lakoff \& Johnson, 1999; Lakoff \& Turner, 1989; Landau, 2000; Matlock et al., 2005), it is commonly held that it is metaphor that helps temporal understanding through spatial mapping, i.e. knowledge of space is used to understand time. Because space and time are two primary domains, this kind of metaphor is called a primary metaphor, e.g. TIME IS SPACE (Grady, 1997; Lakoff \& Johnson, 1999). However, in Embodied Cognition, as mentioned in Chapter 1, space and time are always bound together and take place simultaneously in any event. That is to say, space and time are forever part of any cognitive activity as a whole, and they are inseparable in this sense. In accordance with the cognitive domain view of metonymy and metaphor, when space is used to understand time in this situation, it should be a PART-PART relation, i.e. metonymic, rather than metaphoric. This, from another perspective, shows the potential problem of the domain view. Even if the whole event or scenario/frame/ICM is considered as the super-domain, and space and time as subdomains of this scenario/frame/ICM, as is often done in Cognitive Linguistics, the mapping between SPACE and TIME is a mapping between sub-domains within the super-domain of the scenario/frame/ICM, i.e. it is instead a metonymic mapping. Thus "domain" is confusingly problematic in this view and should be avoided. 
Besides, Embodied Cognition does not occur in terms of domain, but in terms of (inter)action or eventual activity, or rather, event scenario $^{32}$.

Therefore, Embodied Cognition indicates that the cognitive mechanism underpinning the spatial understanding and expressing of time is not necessarily metaphor, as widely held in Cognitive Linguistics, but basically metonymy. This also has a concern in treating metaphor as grounded on metonymy and answering why concepts of space are employed in temporal conceptualization. As mentioned earlier, the source of embodied understanding of the surroundings is indeed the perceptual and sensorimotor activities in interaction with the environment. There exists no interaction that does not happen in a certain spatio-temporal situation. Due to the perceptual property of Embodied Cognition, the salient part is more naturally attended to and focused on. Time is actually noticed or sensed passing by, for example, in the sun shadow's movement from here to there (or in the sun's movement from here or there), or in the feeling of the sunlight's strength. When infants observed an object rolling down from a height and hitting another object to a distance, for instance, their eye fixation lasted longer in the long rolling distance than in the short distance; at once they also observed that the more force the rolling object (e.g. bigger size, wider) inflicted on the stationery object, the more distance the object hit went; still simultaneously, they noticed the longer distance the object hit went, the longer time it went (cf. Wang et al., 2003). Many psychological experiments, as cited in 3.2, illustrate that infants attend to the correlations of parts in actions and events, and this also includes correlation of space and time. They form neural patterns or correlates in frequent embodied experiences of actions and events. Experiments in neuroscience have illustrated at least three kinds of neuron patterns of action, for example, the action-location neurons circuit, which is activated when subjects hear or see objects move in their peri-personal space and which controls movements aimed at objects in peri-personal space; the mirror neurons circuit, which matches action observation, thinking and execution; and the canonical neurons

\footnotetext{
${ }^{32}$ Comprehensively speaking, a cognitive activity generally involves actions, time, space, objects, subjects; it does not exist as a pure action, but a happening in a certain environment. Therefore, it might be more appropriate and convenient to employ event scenario in the cognitive analysis of language and hence be consistent with the integrated analysis advocacy of Embodied Cognition. In this sense, (event) scenario is better than domain in that scenario can be used to well interpret those situations that domain has difficulty in interpreting due to its own problems as a term, as argued in the previous chapter.
} 
circuit, which is activated when observing, naming silently and imagining using various man-made objects (e.g. hammers, screwdrivers) as actually performing those actions (Feldman \& Narayanan, 2004).

These neuron circuits form different neural patterns in embodied experience. Concepts are perceptually grounded, and perception and conception are both processed in the same neural area (Barsalou, 2008a, 2010; Goldstone \& Barsalou, 1998). Research indicates that temporal and spatial processing share the same neural region in the brain, i.e. the parietal cortex region, and input from multiple sensory modalities is integrated in these brain regions (Hubbard et al., 2005). This kind of experimental research, i.e. showing that temporal and spatial information is processed in integration in the same brain region, is often cited as the neural support of the TIME-SPACE metaphor, e.g. Gallese \& Lakoff (2005). What might be ignored is the fact that temporal information is initially embedded and integrated spatially as a whole in embodied interactions owing to the inherent multimodality of perception and sensorimotor activities. Since temporal information is spatially sensed and encoded, there is no mapping between these two, at least in a certain cognitive activity; rather, they are integral in nature.

Therefore, it seems that time is not an autonomous domain, nor is space, though comparatively speaking, space is more salient. The term "aspect" is preferred here in order to avoid the confusing term "domain". Time and space are inseparable aspects of any cognitive activity. It is necessary to make sense of time and space in the framework of Embodied Cognition. As said earlier, time passing can be sensed and observed by through inside feeling and outside perception. For example, after you walked a long distance, you felt hungry; you sensed that there was a difference between your breakfast and your feeling of hunger; but you might not express that it has been a period of time since your breakfast if you have not conceptualized the time. Perception does not need any word but conception has to rely on words/language. Thus you might just say: I am hungry, which best shows that you felt the time interval or time passing. The perception of time might not need spatial contribution though time happens together with space, but in general the conception of time eventually depends upon spatial terms/concepts. It might be said that time and space have their own perceptualization or perceptual categories, but their 
conceptualizations converge in that the conceptualization of time is based on spatial concepts and they are processed in the same brain region. Take the Amondawa (Sinha et al., 2011) as an example: Though their time may be conceptualized through progressive change in social status, which is a little different from what is evident in most cases, i.e. based on the distance or other obvious spatial relations, yet their temporal conception is still based on spatial relations. Their age change is accompanying their name change, together with their social role/status change, since their perspective is on the social role/status owing to their primary farming and hunting life (e.g. mainly dependent on the sun's heat/place, on the moon's position, etc.); their age concept, therefore time conceptualization, is reasonably based on this specific perspective. That is to say, spatial relations have sort of hierarchies, direct and indirect. What is known so far is obviously directly perceived spatial relations such as UP and DOWN, LONG and SHORT, HIGH and LOW; however, there are also other spatial relations which might not be as perceptive and obvious as the above but can be still employed to conceptualize time, relying on the specific cultural perspective or focus.

When "time" is said to be spatially conceptualized, it must be talking about an Embodied Cognitive event/activity, as mentioned earlier, which is the whole guiding the thinking in the situation and to which "space" and "time" are constituent parts. The relation between those constituent parts are hence in nature metonymic, just like the time "10:00" is intended to mean "the person with whom you have an appointment at 10:00" within the APPOINTMENT event (or rather, "10:00" is conceptualized as "the person with whom you have an appointment at 10:00", at least at the moment). In this case, of course, the conceptualization does not necessarily proceed this way; the person might be conceptualized as "the beards", "the bald", or other ways such as place (room/office the appointment is arranged for), the person's personality/peculiarity, the reason for the appointment, etc; it all depends on the actual situation. By the same token, time can be conceptualized this way. Besides the obvious spatial aspect of the Embodied Cognitive scenario it can be conceptualized by other aspects in the scenario, e.g. the change of social status, the change of marital status, etc, as in the Amondawa example. 
In this sense, although spatial concepts/terms are used in the conceptualization of time, they are intended to mean temporal concepts, not spatial concepts. It is essentially a conceptual borrowing. For example, the spatially-conceptualized time in the way cannot be extended as in space. In a word, even though time is made sense of in the way of space, it is not a mapping between time and space, at least when involving a certain cognitive scenario; just like that the "10:00" is understood not as the time but as the person for whom the appointment is arranged at the time. If it is considered as a metaphor, then what similar aspect exists or is created between time and space?

The conceptualization of time on space is not enabled by time and space themselves, but potentiated by Embodied Cognitive event/activity. For metonymic relation, it is not necessary to have specific aspect in time and specific aspect in space related; time and space are inherently related, actually inseparable, within the embodied cognitive activity/event. Different from metaphor which needs at least two aspects of the source and the target to have a similarity, time and space don't need this similarity. That spatial relations (whether direct/obvious or indirect) or other possible relations are employed to conceptualize time is determined by the embodied nature of cognition which takes place in interaction with(in) the physical, social, cultural, lingual, economic environment. As integral parts of an embodied cognitive activity, as in APPOINTMENT case, time and space are metonymically related and conceptualized.

In sum, Embodied Cognition favours temporal-spatial integration. Spatial cognition of temporal information is in essence PART-FOR-PART metonymy, i.e. temporal cognition starting from space is inherently the result of metonymy as a natural cognitive faculty in the embodied cognitive event.

However, it is presumable that the space aspect of one cognitive scenario is employed to understand the time aspect of another cognitive scenario after each experience is conventionalized. In this situation, the temporal configuration in one case based on the spatial configuration in another case is metaphoric. This process carries a further extension beyond the above TIME-FOR-SPACE metonymy in a certain cognitive scenario, building up a metonymy-based TIME AS SPACE 
metaphor. It should be pointed out that it is in this sense that TIME-SPACE mapping applies.

\subsection{Summary}

Metonymy in the cognitive perspective is characterised by a cognitive process relating PART and WHOLE within the same domain/frame/ICM with PARTWHOLE as the prototypical relation between the source and the target. This viewpoint is derived from extensive linguistic analysis of language structure and language use. It is argued in this chapter that metonymy is an innate cognitive mechanism which attentively picks up the salient part to form a whole such as a concept, a category, and a mental event image schema and then relate the PART and the WHOLE, relate different components (i.e. PART and PART). Metonymy not only operates in language-involved conceptual activities, but also in languagebeyond conceptual activities and perceptual activities. All these are demonstrated and evidenced in the cognitive activities of infants and subjects with impaired language ability. In infants' perceptual and conceptual categorizations, and even in children and adults, metonymy is executed to carry out the PART-WHOLE FORMING process, i.e. they form concepts and categories on the base of part of perception of entities, and this is a process from PART to form a WHOLE. Category and concept, nevertheless, develop from WHOLE to PART. The initial categories and concepts are rather general or global without specific subdivisions, but they will get enriched and detailed with embodied experience and cognitive development, encapsulating more members with gradually more specific information and hence forming a category or concept hierarchy. Infants' inductive inference is charged and guided by the kind knowledge of concept or category they acquired through unconscious PART-WHOLE FORMING. They spontaneously employ this WHOLE knowledge to make inference and generalization, to predict and expect what is next or what will happen next. Category and concept development and inductive inference are both sort of PART-WHOLE RELATING process, and specifically from WHOLE to PART, with the WHOLE as the supervisor. Infants' memory, and actually human memory, involves both from PART to WHOLE process and from WHOLE to PART process. Memory is formed and organized on attentive perception of particular part of an event/action/entity, and develops from WHOLE to PART, i.e. 
the WHOLE is in charge of memory development with the salient part as the trigger. Memory breakdown research shows that subjects lose their memory starting from detailed specific bottom information (subordinate) and then basic level, the lasting part of memory is the initially formed general category.

Spatial knowledge is believed to encode time. It is supposed in the domain view that space is mapped onto time in making sense of time, i.e. the mapping from space to time is metaphoric since space and time are different primary domains. However, space and time never exist independently. Embodied Cognition is grounded on perceptual and sensorimotor interactive experiences with the physical, socio-cultural environment; space and time are bound together as an embedment in these interactions. Neuroscience research suggests that the same neural area in the brain is activated in processing both spatial and temporal information. Space and time, even where there is a relation of one encoding the other, are binding components of embodied interactions; from space to time is hereby metonymic in nature in this situation, they are PART to PART RELATING in a certain cognitive scenario.

All these aspects seem to suggest that metonymy is an inherent cognitive mechanism, and it could be said to be the nature of Embodied Cognition. Given metonymy is a natural cognitive mechanism, metaphor, which was viewed as a cognitive process, and even the predominant one, in Cognitive Linguistics, seems to be a natural cognitive mechanism as well; then what is the difference and relationship between these two? This question is addressed in the following chapter. 


\section{Chapter 4 Grounding Metaphor on Metonymy}

Chapter 3 discussed the features of metonymy in the cognitive perspective and provisionally argued that metonymy is an innate cognitive mechanism rather than merely a cognitive process and it operates both perceptually and conceptually in two stages. In the first stage metonymy operates to form a WHOLE from PART in perceptual and conceptual categorization, and then in the second stage it relates PART and WHOLE/PART in category and concept development, inductive inference, and memory under the control of the formed WHOLE. The previous chapter is mainly devoted to research in infants and language-impaired subjects whose cognitive activities are the least influenced by language. When both are viewed as a cognitive process in Cognitive Linguistics, metonymy and metaphor are hotly discussed in terms of mutual relations in the linguistic analysis of idioms, phrases and lexical semantics. This chapter is targeted to the cognitive analysis of the relationship between metonymy and metaphor and to the probationary argument that metaphor is conceptually grounded on metonymy and that a metaphoric operation presupposes at least a metonymic operation.

\subsection{Metonymy versus metaphor: A review}

As mentioned in Chapter 2, in traditional rhetoric since Aristotle, metonymy is accommodated under the label of metaphor as a special case of it; metonymy is inferior to metaphor in study in terms of popularity, and is less, if at all, researched in the form of books and monographs. Metonymy is not given the same standing as metaphor until historical semantics, in which metonymy is put at the same level as metaphor for the first time as one of the four mechanisms of lexical meaning change, together with synecdoche and irony. But only metonymy and metaphor are kept as the most significant mechanisms of semantic extension. The relation between metonymy and metaphor has been in scholarly dispute ever since. Structuralists and semioticians argue that metaphor is based on an iconic relation between the source and the target while metonymy is based on an indexical relation.

Metaphor and metonymy began to enter the study of language structure as well as literary works. Metonymy was considered to dominate in prose while metaphor was 
primary in poetry. Metonymy and metaphor as two kinds of relation are considered omnipresent in language. Jakobson (1971b, p. 244) thought that "language in its various aspects deals with both modes of relation". He even found that metonymy and metaphor were related to mental disorders, e.g. aphasia. Jakobson focused on metonymy and metaphor separately and considered metaphor as selection or substitution and metonymy as combination or contexture. However, either selection/substitution or combination/contexture takes place on the basis of syntagmatic or linear construction. The eligibility and selection of substitutes has to depend on the syntagmatic construction of the utterance; and the possibility of combination of constituents has to rely on the linear construction; and these happenings have to comply with the context of utterance as well, i.e. the external relation of contiguity, in Jakobson's term.

Rekindled by Lakoff \& Johnson (1980), research on metonymy and metaphor in recent decades has witnessed both extensive and intensive effort to distinguish them from each other. But most of the discussion is on similarity in metaphor and contiguity in metonymy, as discussed earlier in Chapter 2. Metaphor is commonly considered to be based on a similarity/iconic relation and metonymy is on a contiguity/indexical relation.

However, as Lakoff \& Johnson (1980, p. 148) themselves point out, what makes similarity is metaphor itself. In addition, as Warren (1998) demonstrates, some clearly iconic cases are metonymic (see Chapter 2 for examples). She suggests that metaphor is a kind of feature connection and metonymy is a kind of relation connection, i.e. in metaphor the connection between the source and the target is one or more features but in metonymy it is a predictable relation. Thus the interpretation of metonymies involves retrieving a relation and interpretation of metaphors involves retrieving at least one attribute shared by the referents (Warren, 1999); metonymy expresses implicit modifier-head construction which involves no substitution, while metaphors involves property transfer (Warren, 2002). In Haser's (2005) view, the knowledge of the target does not imply knowledge of the source in metaphor, while in metonymy the source and the target senses are inseparable in principle, i.e. metonymy is class-inclusive between the source and the target (but see $\mathrm{Fu}, 2010$ for a review). 
Most recently, it has even been suggested that it would be more profitable to analyse utterances at the level of the dimensions involved in the differences rather than metaphor and metonymy as such, since metonymy partly involves similarity and metaphor involves contiguity (Barnden, 2010).

The discussion of similarity and contiguity even goes so far as to take them as complementary rather than mostly held opposite relations, i.e. contiguity is regarded as non-similarity relation (see e.g. Feyaerts, 2000; Ullmann, 1962).

Another trend is to employ different terms to define the relations between the source and the target in both metonymy and metaphor. The most popular view is the cognitive domain approach to metonymy and metaphor, which is considered to serve best in distinguishing metonymy from metaphor. Metaphor hereby is a mapping between different domains, i.e. inter-domain mapping, and metonymy is a mapping within a domain, i.e. intra-domain mapping. It seems successful to serve the purpose, but as shown in Chapter 2, the terms ad hoc used here are as problematic as "contiguity" and "similarity" used before. First, "domain" is a rather ambiguous concept, intra- and inter- domain is of no use to make distinctions. Second, "mapping" is employed to define both metonymy and metaphor, which indicates that metonymy and metaphor work in the same way.

Studies of metonymy and metaphor in lexical semantics suggest that it is hard to have a clear cut distinction between metaphor and metonymy. For example, Goossens $(1989,1995,2002)$ find that there are several integrations between metaphor and metonymy, such as metaphor from metonymy, metonymy within metaphor, metaphor within metonymy, and demetonymization inside a metaphor, and he terms these interactions between metonymy and metaphor as metaphonymy. Dirven (2002, p. 93) considers metonymy and metaphor both as linear in conceptualization, i.e. viewing metonymy and metaphor as a metonymy-metaphor continuum from non-figurativeness to complex figurativeness. Ever since, the metonymy-metaphor continuum view has been held and supported, and metonymy and metaphor are hereafter seen as opposing poles with a middle fuzzy area covered by metaphonymy, or by metonymy-based metaphors (Radden, 2000, 2002; Taylor, 2002a). 
Research on the interaction of metaphor and metonymy in language and cognition also suggests that metonymy is as ubiquitous as metaphor (Barcelona, 2002b; Panther \& Thornburg, 2003c; Radden, 2005); metonymy is primary, and it is more central than metaphor (see e.g. Barcelona, 2000b; Dirven \& Pörings, 2002; Gibbs, 1994; Koch, 1999; Mittelberg \& Waugh, 2008; Mittelberg \& Waugh, 2009; Sugeno, 1996; Taylor, 1995, 2002a); and metaphor is often metonymy-motivated or based (Barcelona, 2000c, 2002b; Gibbs, 1994; Kalisz, 2007; Radden, 2005; Taylor, 1995, 2002a).

The relationship between metonymy and metaphor is thus becoming more confused and complicated. So, what is the exact relation between metonymy and metaphor in the conceptual perspective? Is it really a continuum that metonymy and metaphor form? This chapter aims to answer these questions. It suggests that metonymy is probably what makes metaphor possible in cognition, and metaphor seems to be conceptually grounded on metonymy, although both of them are natural cognitive mechanisms. Following the previous chapter, this chapter still focuses on languagebeyond cognitive activities.

\subsection{Metonymy in cognition}

Metonymy in language-beyond cognitive activities has been discussed in the former chapter; nevertheless the following has to be emphasized here once again for the convenience of further argument.

In Cognitive Linguistics cognition is considered as embodied, and reality is supposed to be constructed by the nature of the unique human embodiment, and the construal of reality is likely to be mediated by the nature of the bodies (Evans \& Green, 2006). What is perceived is only part of the entity, part about the entity. In conceptualization and categorization, the entity is taken as a general WHOLE. Thought is contended to result from the ability to act in the environment. It means that cognitive actions are goal-directed in certain settings, which further indicates that in cognitive actions attention is just paid to what is meaningful to the observer. Looking can be done without seeing; and "inattentional blindness is probably the fate of much of the perceptual information human beings process" (Mandler, 2004a, p. 69). 
Embodied Cognition is innately generalizing and abstracting. Humans are born with the capacity to categorize and conceptualize the world and to bring aspects of the perceptual world to conscious awareness. The perceptual system and conceptual system develop simultaneously from birth (Mandler, 2004a). Humans are also born with the capacity of searching for relations (which therefore determines the ability of generalization and abstraction), relating entities beyond themselves, relating entities with themselves, through actions. "Actions are central in organizing the beginning conceptual system from birth" (Mandler, 2006, p. 111). The initial conceptual categories are general and global, and conceptual development frequently goes from the global to the specific (Mandler, 1999; Mandler \& McDonough, 1998; Quinn \& Eimas, 1998; Quinn \& Johnson, 2000). The conceptual process from the global/general to the specific is unavoidably metonymic in the core sense of metonymy in rhetoric, in historical semantics, in structural linguistics, and in Cognitive Linguistics.

Concepts are formed and represented as prototypes (Rosch, 1975). A prototype is an organized set of knowledge that reflects the best example of a category (Trenholm \& Jensen, 2004, p. 144). Humans have an insatiable appetite for categorization (Trenholm \& Jensen, p.140). The capacity to categorize is the most fundamental of human capacities (Lakoff, 1987b, p. 8). Categories do not have criterial features but harbour prototypes, with less prototypical members being apprehended with reference to the extent that they resemble (or fail to resemble) the prototype (Gardner, 1985, p. 346). Prototypicality exists because it is cognitively advantageous "prototypical categories carry both structural stability and flexible adaptability: On the one hand, the categorical system can only work efficiently if it can maintain its overall organization for some time; on the other hand, it should be flexible enough to be easily adaptable to changing circumstances; prototypically organized categories are particularly well suited to fulfil this double demand for flexible adaptability and structural stability" (Geeraerts, 1988, p. 227).

The reality is constructed by structuring, stabilizing, and relating the stream of stimuli around in meaningful ways (Trenholm \& Jensen, 2004, p. 146). Those that are meaningful are kept in mind, but only part is recalled from the mind to construct the memory according to what attention is paid to in the current situation. Memory is 
actually a process of construction, it is the dynamic interplay between inner (processes) and outer (settings) (Engel, 1999, pp. 6-8). People do not reproduce from memory what was but at least partly reconstruct what was, based on what must have been (Trenholm \& Jensen, 2004, p. 144). All these dynamic cognitive activities seem to be dominated by metonymy with PART-WHOLE or PART-PART RELATING. This can be demonstrated in the following quote from Kwiatkowska (2007, p. 297):

The information that we obtain in the process of perception is practically always incomplete. We have a limited field of vision, and an object may extend beyond it, so we see only its portion; we cannot view an object from all sides at once; parts of objects are also usually hidden from view by other objects. We see the spine of a book standing on a shelf, someone's leg sticking out from under a blanket, a photograph which shows only a person's face. We look out of the window and see a small fragment of the street, a bus momentarily coming into the frame to disappear from view again. Yet we are all able to infer automatically from this partial information that there is a complete book standing on the shelf, and that a whole person is sleeping under the blanket; we do not interpret the photograph as showing a cut-off head, or conclude that the street is ten meters long and that the bus can cover its route in several seconds.

Furthermore, as mentioned previously, according to Mandler (1998b, pp. 147-169), human memory, just like their conceptual system formation, is also organized in a hierarchical way, i.e. from the global or super-ordinate to the specific. An entity is usually conceptualized as a member of a general/global or super-ordinate class; the association of features to objects in semantic memory occurs via the super-ordinate. The data on acquisition and breakdown of the semantic system suggest that superordinate categories are more resilient and longer-lasting than more detailed subdivisions, and the super-ordinate is the "first in, last out", because more detailed concepts are constructed out of super-ordinate ones and their most fundamental meaning stems from their super-ordinate class membership. Therefore it seems that every aspect of human cognition is innately a PART-WHOLE, WHOLE-PART, contiguous relation, i.e. metonymic in nature, which advances with and underlies the acquisition and use of language. So the conceptualization, categorization and 
memory might in essence function metonymically, as argued in the preceding chapter.

All in all, Embodied Cognition appears to be metonymic in nature, and it starts from PART to form a WHOLE and develops from the WHOLE to PART or between PART and PART.

\subsection{Metaphor in cognition}

Whether metaphor is taken as a figure of speech in old rhetoric or as a mechanism of semantic change in historical semantics and structural linguistics or as a figure of thought/ cognitive process in Cognitive Linguistics, metaphor is considered to take place between two different entities based on comparison, contrast and similarity. But where does the similarity or similar aspect come from? From the perspective of cognition, it definitely comes from the entities that have been separately perceived and conceptualized, i.e. this similarity relation between the two entities originates from them as separately perceived individuals. As argued in section 4.1 and the previous chapter, that the specific aspect(s) of an individual is inherently used to represent or mean the individual as a WHOLE in cognition is totally metonymical, and this aspect of the individual is then employed metonymically in place of the whole individual to form a similarity relationship with another individual entity, thus creating a metaphor. That is to say, metaphor as a cognitive process is formed on the operation of metonymy in cognition.

The analysis of metaphor in LCCM theory, for instance, shows that metaphor is an interpretative process in which integration guides the access to the secondary cognitive model profile through the first cognitive model (Evans, 2007; Evans and Zinken, to appear) (for details of LCCM theory, see (Vyvyan Evans, 2006, 2007)), and this access is definitely provided by metonymy. This is also why Barcelona (2000c, p. 31) claims that "the target and/or the source must be understood or perspectivized metonymically for the metaphor to be possible".

So in this sense, it might be claimed that conceptually metonymy and metaphor do not form a continuum as announced in Cognitive Linguistics, but rather they form a hierarchy with metonymy as the base and metaphor as a derivative from metonymy. 
This point can be illustrated from the perspective of semiotic cognition, as shown in the following diagram (Figure 4.1). Entity ${ }^{33} \mathrm{~T}$ (target) involves many properties, features, functions, etc. Such as T1, T2, T3, T4 ..., Tn. Entity S (source) may as well have many properties, features, functions, etc. Such as S1, S2, S3, S4 ..., Sn. All these are aspects of the entity. The diagram shows that in order to form a metaphor, the source entity needs to be conceptualized before the target in principle. As mentioned above in section 4.1, human conceptualization is metonymic in nature, memory is dynamic. Memory is reconstructed according to what is meaningful (thus attracts attention). It is a network of meaning. The working system of memory is always searching for meaningful connections with outer settings. Thus when attention is paid to $\mathrm{T}$, at least one aspect of $\mathrm{T}$ will be obtained, say, $\mathrm{T}$, , that is meaningful to $S$ (actually to some aspect of S, say, S') in terms of similarity. Once this similarity relation is established in cognition, the metaphor T IS $\mathbf{S}$ is formed. Therefore from the forming procedure of metaphor, it is obvious that metonymy plays a vital role in metaphor derivation.

Figure 4.1: The formation of metaphor from the perspective of semiotic cognition

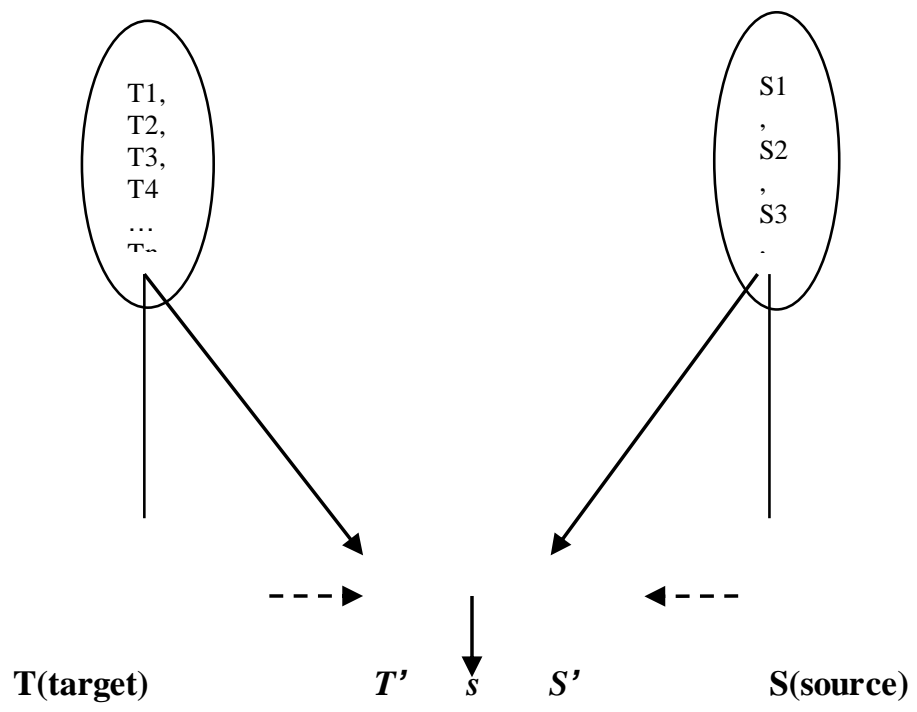

\section{$\mathbf{T}$ IS $\mathbf{S}$}

(The long solid arrows express "perspectivization"; the broken arrows express "representing metonymically"; the short solid arrow expresses "forming"; the small "s" means "similar in relation")

\footnotetext{
${ }^{33}$ Here, Entity is used in the broad sense, covering categories, frames/ICMs. That is, the source/target may be a category, or a frame/ICM, or a member of them.
} 
The above interpretation is from the normal cognition. In order to see the metonymic base of metaphor, the following paragraphs will examine one aspect of the abnormal cognition, i.e. aphasia.

Jakobson (1971) treats the similarity disorder and the contiguity disorder as two different types of aphasia. He places these two as opposite patterns and considers the first as deficiency in selection and substitution and the latter as deficiency in combination and contexture. Jakobson thus posits that "every form of aphasic disturbance consists in some impairment, more or less severe, either of the faculty for selection and substitution or for combination and contexture" (Jakobson, 1971, p. 254). If metonymy was the base of metaphor, then it would be necessary to relate these two disorders of aphasia in some way and give a rational interpretation for the proposed relationship between metonymy and metaphor in aphasia.

According to Jakobson, for the type of aphasia he calls similarity disorder, contiguity determines the patient's whole verbal behaviour, e.g. "Fork" is substituted for "knife", "table" for "lamp", "smoke" for "pipe", "eat" for "toaster"; for the opposite type of aphasia, contiguity disorder, the ability of combining words into a syntactic construction is impaired, only content words are used, forming the so-called telegraphic style and diminishing the extent and variety of sentences, e.g. "fire" for "gaslight" (i.e. the general for the specific).

Since the similarity disorder aphasia is taken as metonymic in nature, only the contiguity disorder aphasia will be discussed here. Jakobson treats the contiguity disorder as metaphoric. This kind of patient cannot dissolve linguistic units such as words and sentences. For example, Jakobson points out that this kind of patient uses "spyglass" for "microscope" and "fire" for "gaslight". The relationship between "spyglass" and "microscope", between "fire" and "gaslight" is supposed to be a similarity relation in Jakobson's view, i.e. microscope is (like) spyglass in some way, and gaslight is (like) fire in some way. But here two aspects have to be taken into account. When the patient uses "spyglass" for "microscope" or "fire" for "gaslight", (s)he must have already in mind the presupposed propositions MICROSCOPE IS LIKE SPYGLASS and GASLIGHT IS LIKE FIRE in order that the metaphoric utterance (or inference) can be made. This is a linear construction in cognition and is formed through a metonymic cognitive process; otherwise the patient cannot utter 
"spyglass" to stand for "microscope" and "fire" for "gaslight". That is to say, this kind of aphasia is based on metonymy.

The other aspect is the complexity of aphasia. It is obvious that Jakobson simplifies both the problem of aphasia and the classification of aphasia. Aphasia involves many aspects such as inability to comprehend language, inability to pronounce, inability to name objects, inability to form words, inability to read, inability to write. But aphasia is not a loss of intelligence. So it is necessary to consider both the conceptual aspect and the verbal aspect. Ciepiela (2007, pp. 199-208) made a study of metonymy in aphasia involving both conceptual and verbal parts. The experiment results show two significant points: One is that the experiment confirms that metonymy is both a cognitive and a language phenomenon, poor performance on conceptual tests coincides with low efficiency in the language test; the other is that contrastive patterns of language breakdown do not exist in the way that Jakobson claims. In aphasia both metonymic and metaphoric mechanisms are impaired to some extent. Nevertheless research on aphasia suggests that metonymy is the primary mechanism, i.e. a mechanism which is more primitive, experientially grounded, metonymy and metaphor operate in parallel and metaphor is more restricted in aphasia. This indicates, from the perspective of Embodied Cognition, that metonymy is more perceptually grounded and metaphor relies on metonymy, though they operate as two cognitive mechanisms. But in the case of aphasia, more experiments are needed to verify or falsify this claim, e.g. to check whether there is any case where the metonymy mechanism is damaged and the metaphor mechanism remains intact.

Another aspect that shows metaphor as metonymy-based in cognition is to look at conceptual metaphors as put forward by Lakoff \& Johnson (1980). Lakoff \& Johnson (1980, p. 4) conclude that the following linguistic expressions derive from the shared conceptual metaphor ARGUMENT IS WAR:

- Your claims are indefensible.

- He attacked every weak point in my argument.

- His criticisms were right on target.

- I demolished his argument.

- I've never won an argument with him. 
- You disagree? Okay, shoot!

- If you use that strategy, he'll wipe you out.

- He shot down all of my arguments.

They think that the reason of talking this way is because the thought is shaped by the conceptual metaphor ARGUMENT IS WAR (this is in the sense of their saying "what makes similarity is metaphor itself"), which determines the talking and thinking about arguments in terms of war. They therefore claim that the ordinary conceptual system is fundamentally metaphoric in nature.

Despite the logic of their claim, the immediate question to ask is: If there are conceptual metaphors, how and where does this kind of conceptual metaphor come from? It is certainly not inborn. It comes from the separate embodied experiences of WAR and ARGUMENT, and the separate conceptualizations of WAR and ARGUMENT, which is each processed metonymically, as analysed below.

To form the so-called conceptual metaphor ARGUMENT IS WAR, it is necessary to conceptualize WAR and ARGUMENT separately and probably in a successive way (because this conceptual metaphor goes in a certain direction, i.e. from WAR the source to ARGUMENT the target), both of the conceptualizations are formed out of specific aspects of WAR and ARGUMENT respectively, and then some similarity connection between these two concepts is found due to some functional factors of the specific context prior to the formation of the conceptual metaphor ARGUMENT IS WAR.

So it is obvious that the conceptual metaphor Lakoff \& Johnson identify is in the second stage of the cognitive processing, it is a second inference or reasoning based on a metonymically formed WHOLE of WAR and ARGUMENT respectively. Conceptually WAR and ARGUMENT are formed metonymically as a WHOLE from specific aspects/properties (PART) of war and argument respectively, and then these two concepts are combined into a metaphoric formula. If this is the case, it is likely that metaphor is grounded on metonymy, and human perceptual system and conceptual system are fundamentally metonymic in nature. Metonymy is probably the default mechanism in this kind of cognitive activity, and metaphor is a secondary formation, depending on metonymic operations. 


\subsection{Metonymy and metaphor in the interactive perspective}

Chapter 2 suggests that metonymy and metaphor in the cognitive domain view are not actually clearly defined and distinguished since the existence of the blurry terms of "mapping" and "domain". From the analysis of 4.3 , it is clear that metonymy and metaphor do not form a conceptual continuum but rather they form a conceptual hierarchy. Based on this viewpoint, it would be necessary to clarify metonymy and metaphor according to how they function in cognition and in communication so as to have a thorough understanding of metonymy and metaphor as cognitive mechanisms in interactive communications. However, as a cognitive mechanism, either metonymy or metaphor is only realised in some linguistic representation and recognized in language. So in order to illustrate how they function in the interactive perspective, the following will be related to language use to some extent.

Lakoff \& Johnson (1980, p. 36) think that the primary function of metonymy is referential and the primary function of metaphor is understanding, but they point out at once that metonymy is not merely a referential device, it also serves the function of understanding. Therefore, both metonymy and metaphor serve the function of providing understanding and providing reference, in Lakoff \& Johnson's terms, by highlighting certain aspects of what is being referred to. While Lakoff \& Johnson stress the understanding function of metaphor, Dirven (2002, p. 105) places more emphasis on the expressive function of metaphor, he connects the representational or referential function with metonymy and the expressive function with metaphor. As is well known, language as a system of symbols is referential (i.e. indexical) in nature; it mediates as a referential servant between the mind and the environment. As Tomasello (2003, p. 8) points out,

\footnotetext{
"Linguistic symbols are social conventions by means of which one individual attempts to share attention with another individual by directing the other's attentional or mental state to something in the outside world."
}

Whether it is used to understand or to express, it hence could be said that the understanding function or the expressive function derives from the elementary referential function, which also implies that metaphor is derived from metonymy in language. 
From the perspectives of the origin of language and cognitive development, it seems that language is not primarily used to conceptualize, but rather to express; to use language to refer to what is intended to express, to be specific. That language is meaningful relies on what to do with language. From the perspective of developmental psychology, especially cognition development and language development, according to Mandler and her colleagues (Mandler, 2004a, 2004b; Mandler \& McDonough, 1993), babies have to have some conceptual base developed first for the development of their language. Before infants start to learn language, they have developed some cognitive prerequisites: A rich understanding of the external world, the ability to infer the referential intentions of others, the ability to use and understand motivated signs (both icons and indices), the ability to imitate (Burling, 1999). They do think before they have language (Mandler, 1998a). Infants form global concepts from what is meaningful to them before they acquire language. In this sense, their understanding is surely before they acquire language. But language is undoubtedly a great enhancing device for cognition. For example, pain can be experienced without language, but the recall, referring and thinking about the experience has to resort to language, because how it is felt cannot be otherwise reexperienced (Mandler, 2009, personal communication). Thus in this sense, language seems to be referential from the very beginning and it is primarily used to express and do things.

Cognitive Linguistics stresses the conceptual aspect, neglecting the expressive aspect of language, to be specific, of metonymy and metaphor. Although recent research in psychology suggests that there is no principled distinction between language comprehension and language production (Wilshire, 2009, personal communication), yet there may exist distinctions between what one thinks and what one utters, between the speaker and the listener. Since human cognition is embodied, according to the Embodied Cognition Theory, cognition is inevitably primarily goal-directed (Cowart, 2009). Besides inner conceptual activities, expressing activities are common among humans as well. What is uttered is not always necessarily what is actually in the mind, especially in communication. People are sometimes doing "thinking for speaking" (Slobin, 1996). Thus it might be better to take into account the expressive aspect as well as the purely conceptual aspect of language (Geeraerts, 1988, p. 225). Language is used for doing things; language use is really a form of 
joint action (Clark, 1996, p. 3). Language is not employed to merely conceptualize the world but is employed by the speaker to express himself so as to communicate with others. That is to say, the other aspect of metonymy and metaphor as cognitive mechanisms, i.e. their expressive aspect, should also be taken into account, especially when dealing with interactions in dialogue, i.e. their functions in interactive communication.

In interactive communication, i.e. in dialogic discourse, the speaker and the listener undergo different experiences in expressing and comprehending metonymy and metaphor. The speaker, before uttering a metaphor, has already perceived the similar aspect(s) between the source and the target and understood them quite well in the situation. So this process consists of perceptualizing and conceptualizing the source and the target separately and then finding the similar aspect(s) between them, in which metaphor is not necessarily involved. Sometimes it is only when it is necessary to describe/conceptualize something with the help of something else that the metaphoric process operates, it is sort of "thinking for speaking" (Slobin, 1996). In this sense, metaphor is a later process which operates after making sense of the source and the target separately. In this situation of language communication, metaphor on the part of the speaker is not primarily used to understand something as Lakoff \& Johnson (1980) claim, but rather to express his understanding of this thing (which undergoes a metonymic operation). There are also situations in which nonlinguistic metaphors may be involved, i.e. metaphors may not be verbalized. In this case, metaphor is probably employed to clarify the understanding of the target, which is based on the preceding understanding of the source and the target.

But for the listener in the situation of dialogic interaction, what he has to do when he hears the metaphor uttered by the speaker is first to figure out what the similarity is between the source and the target according to the utterance and the situation. Only after he figures out the similar aspect(s) between the source and the target that is created by the speaker can he understand what the speaker means in using the metaphor and respond in the way he considers appropriate. The metaphor from the speaker invites understanding and pragmatic inferences from the listener. So on the part of the listener, the main process is to make sense of the metaphor the speaker has uttered and make a proper response. Therefore, in dialogic discourse, it seems 
that metaphor is used to express or interpret his conceptual configuration of the similarity relation on the part of the speaker, and to make sense of the speaker's configuration of the similarity between the source and the target and make a corresponding reply on the part of the listener. They are quite different cognitive processes in employing metaphor (in utterance) and in understanding metaphor in dialogic discourse.

When metonymy is used in the speaker's utterance, it suggests that the speaker has already conceptualized the WHOLE, using a PART to represent another PART, or to represent the WHOLE, or vice versa; and at the same time he assumes that the listener might have had the same experience as him. The reason for this metonymic conceptualization is that the very PART employed is the most meaningful to the speaker in the specific setting. ${ }^{34}$ In cognition human beings are always attentive to what is meaningful to them in the particular setting. On the part of the speaker, metonymy is a cognitive mechanism, consisting of perceiving, identifying (a specific aspect of a patterned experience triggers the whole or some other aspects within the whole pattern), and reasoning. It is a specific perspectivization of the conceptualization process of the speaker, which is generally based on common experience. This perspectivization of cognition reflects the speaker's standpoint, emotion, illocutionary act, attitude, etc. It is employed in utterance to the listener whom the speaker assumes has had the same or similar experience in the shared setting. So in principle the listener undergoes the same experience in cognition as the speaker based on the shared knowledge and common experience, because the use of metonymy in dialogic discourse presupposes that in the speaker's eyes the listener has had the same or at least similar conceptualization of an experience as the speaker in the common setting of cognition. For example, in the movie "Point Break", when the FBI special agent Johnny Utah goes to see the regional commander, the receptionist picks up the phone and says to the head: "Your nine o'clock appointment is here." The head replies: "Let him in." The receptionist uses "nine o'clock appointment" for Johnny (i.e. TIME OF THE EVENT FOR THE PERSON

\footnotetext{
${ }^{34}$ Here, "meaningful" is not used in the linguistics sense, but in the cognitive sense. Following Mandler (2009, personal communication), "meaningful" is meant to be what makes it predictable and memorable, which lies in what we do with the material in question, not something that resides intrinsically in this or that thing.
} 
THE EVENT IS SET FOR), for in her cognition, what is the most meaningful to her as a receptionist is the time of appointment, which is prior to the name of the person although she knows it as well. Therefore her conceptualization is specifically perspectivized on the time of appointment with the name of the person in the background. The listener in this case can easily know what she means for he has the same or similar cognitive experience already (anyway it is a pre-arrangement). It is a kind of ready-use in cognition and a pick-up in utterance.

Some might see metonymy as associative thinking and metaphor as analogical thinking. But specifying the difference between associative thinking and analogical thinking is no different from confusing metonymy with contiguity and metaphor with similarity, if the relation between association and analogy is taken into account. First, analogical thinking, which is based on the analogy (i.e. similarity) between two entities, is basically a special type of associative thinking. That is to say, analogy is a special association which is based on the similarity between two entities. This is why some scholars indicates that the similarity link is also a contiguity relation, e.g. Warren (1995) and Barnden (2010). Second, association, when differentiated from analogy, is still rather broad and seems to cover all relations except analogy; this view is like the view of contiguity and similarity held by those scholars such as Ullman and Feyaerts who take contiguity as non-similarity relation. Third, associative thinking, in this sense, seems to only take place within a WHOLE (patterned experience), but if so, associative thinking does not cover the PARTWHOLE FORMING stage, which is argued in this thesis to be the precondition of PART-WHOLE RELATING (i.e. associative thinking), and thus cannot interpret why a WHOLE can be formed only from a very specific case, e.g. category, concept. If metonymy is not found to be vital or present in the most essential cognitive activities such as categorization and conceptualization, how can it be said that metonymy is a cognitive process or model and ubiquitous in language and cognition for referring and understanding?

What's more, in employing metaphors, the speaker tends to keep his perceived/conceived similarity or similarities between two entities unspecified, leaving it or them for the listener to figure out. For example, "All marriages are iceboxes." (Glucksberg \& Keysar, 1993) The similarity relation between "marriages" 
and "iceboxes" is quite clear to the metaphor creator (i.e. the speaker), but he uses this metaphoric formula to generate a contrast and comparison between "marriages" and "iceboxes", with the assumption that the listener is able to figure out what this contraposition is based on. In other words, by the metaphoric expression the speaker tends to keep the key to the relation between "marriages" and "iceboxes" covered but saliently presupposed in the eye of the speaker. The listener is expected to infer what is presupposed and covered. However, in employing metonymy, the speaker just picks up the salient aspect of the entity (in his view) for the entity proper; this salient aspect is generally also obvious or at least overt to the listener in the speaker's view. For instance, "Senior midfielder Simon Elliott hopes the local factor might galvanise the All Whites to victory in Wellington ..." Here "the All Whites" is used metonymically for the New Zealand national football team. This point is obvious, at least in the speaker's mind. Therefore, metaphor seems to give you the end result from which to infer the presupposition or reason why they are thus formed. While metonymy seems to offer you the obvious clue (at least in the speaker's view) to infer the target, with the formula relation between the clue and the target already established and well-known (still at least in the speaker's mind) in a certain cognitive scenario; if there is any inference, it is an interaction between PART-WHOLE FORMING and PART-WHOLE/PART RELATING.

The essence of metonymy in cognition is initially to pick up a salient PART in the situation to form a WHOLE (e.g. concept, category, event scenario, ICM) and then to relate PART and PART or PART and the WHOLE in inference and memory. Most of the cognitive linguistic study on metonymy is focused on the second PARTWHOLE RELATING phase. Therefore from the perspective of cognizing and expressing (utterance), metonymy might be viewed as a cognitive mechanism of perceptualizing, conceptualizing, identifying an entity from its aspects, and reasoning one in terms of another based on an established embodied experience pattern. Metaphor is a cognitive mechanism that takes place after a metonymic operation; it is a second stage cognitive operation of interpreting and reasoning one 
(the target) in terms of another (the source) based on the presupposed perception/conception of similarity or similarities between them. ${ }^{35}$

In cognition, metonymy is inductive in nature, from the specific to the general. For example, the English Premier League football team Liverpool always wear an all-red home kit, thus they are called "The Reds" metonymically. The same applies to the team Chelsea; it is metonymically called "The Blues". But metonymy is also abductive and deductive when the reasoning is from the general to the specific. Whereas metaphor, as shown in section 4.3, is merely a further inductive reasoning between two entities, based on a metonymic operation.

\subsection{Summary}

People perceive the world around them, but they only bring part of it to their attention and to their consciousness. Their conceptualization of motion, object, event, is based on their specific perspectivizations of them. It suggests that human cognition is inevitably metonymic from the very beginning. Humans categorize entities as prototypes through generalization and abstraction from infancy. They associate features to entities via the super-ordinate, the super-ordinate is crucial for the formation of specific concepts in the first place (Mandler, 1998b, p. 167). Humans keep what is meaningful in memory and relate what is meaningful in the current setting to those in memory; they thus reconstruct their memory metonymically according to their attentive perception and conception in the specific situation. Metonymic cognitive processes are the default processes, on which metaphor is grounded and formed. Humans form concepts of entities through metonymic perspectivizations of the entities as individuals; they form metaphors through comparing and contrasting specific metonymic perspectivizations of individual entities. In cognition metonymy and metaphor do not form a continuum;

\footnotetext{
${ }^{35}$ As mentioned earlier, since metonymy is taken as a natural cognitive mechanism in this research, distinctions are not made between novel metonymies (metaphors) and conventionalized metonymies (metaphors). The results, whether conventionalized or not, are beyond the focus of this research. However, even a metaphor is conventionalized, it cannot be ignored that its production is due to the metaphoric mechanism or process. For those who are familiar with a conventionalized metaphor, it does not impose a problem of understanding, because they are exposed to the use so often that the declarative knowledge is processed as automatic procedure which needs no more mental effort. But for those who are exposed to the use for the first time, e.g. second or foreign language learners, more mental effort is needed. Either way, the mechanism still operates; it is a problem of familiarity with and frequency of that use.
} 
rather they form a hierarchy with metonymy as the base and metaphor as the derived. Therefore, diachronically the relation between metonymy and metaphor undergoes the development from a two-axis to a one-dimension continuum, to a threedimension cubic hierarchy.

As different cognitive operations, metonymy and metaphor have different functions. But it is necessary to take into account both the conceptual function and expressive function. Metonymy is used to understand and express on the part of both the speaker and the listener due to the assumed common experience. Metaphor is employed to express his conceptual configuration on the part of the speaker; the listener has to use the formed metaphor to understand what the speaker intends to express by searching for the similarity. Therefore metaphor is used to understand on the part of the listener. Thus metonymy is an operation of both conceptualizing the entity from a certain perspective and expressing this conceptualization; metaphor is an operation of both reasoning (based on metonymy) and expressing this reasoning, it is a further inductive reasoning from metonymy.

Metonymy in the mind and the relation between metonymy and metaphor in cognition, especially in language-beyond cognitive activities, have been argued in this chapter and the preceding two chapters, which suggest that metonymy is a natural cognitive mechanism and inherently crucial in cognition. The ubiquity and significance of metonymy has been discussed and demonstrated in the literature of Cognitive Linguistics (e.g. Allan, 2008; Barcelona, 2000b; Dirven \& Pörings, 2002; Panther \& Radden, 1999; Panther \& Thornburg, 2003b; Panther et al., 2009; Ruiz de Mendoza \& Otal Campo, 2002). However, there is still more to be explored and further researched, e.g. the empirical evidence on the distinction between metonymy and metaphor is needed from psychology and neuroscience, and further studies of metonymy in language. PART TWO contributes to some of the linguistic field such as grammaticalization, meaning prototype, and nicknames. 


\section{PART TWO METONYMY IN LANGUAGE}




\section{Chapter 5 Metonymy and Grammaticalization}

PART ONE focused on metonymy in the mind with a review of the conceptual perspective on metonymy in Cognitive Linguistics and arguments for metonymy as an innate cognitive mechanism and as the ground of metaphor. Metonymy is contended to be the most essential cognitive mechanism of attentively picking up the most salient part to conceptually form a whole pattern (such as concept, category, image schema, and event scenario/schema) and accordingly relating the PART(s) and the WHOLE or PART and PART. PART TWO is devoted to metonymy in language. Although metonymy has been studied in rhetoric as a figure of speech and in Cognitive Linguistics as a cognitive process and thus is regarded as ubiquitous in language, there are still some areas to be explored and to be further researched in terms of the cognitive function of metonymy. In this part, it will first discuss metonymy in language as a system, i.e. how metonymy as a natural cognitive mechanism plays its role in shaping language and language development, e.g. grammaticalization, then go on to consider factors motivating meaning change, e.g. meaning prototype, and language use, e.g. nicknames. The current chapter will centre on metonymy in grammaticalization.

\subsection{Grammaticalization as a process}

The notion of grammaticalization was actually first recognized in China in the Song Dynasty (960-1279 A. D.), hundreds of years earlier than French linguist Antoine Meillet coined the term "grammaticalization", and even several hundred years earlier than scholars such as Etienne Bonnot de Condillac and Jean Jacques Rousseau had the similar idea (see e.g. Heine et al., 1991a; Hopper \& Traugott, 1993; Sun, 1996). The notion of grammaticalization in Chinese is xulhua4 (emptied), e.g. the Song scholar Lu Jiuyuan said, "zi4 zhi1 zhi3 guil you4 you3 xu1 shi2, xu1 zi4 dang1 lun4 zi4 yi4, shi2 zi4 dang1 lun4 suo3 zhi3 zhi1 shi2" "36 (on the other hand, the meanings of words can be either grammatical or substantive. A grammatical word of course refers to the meaning of the word in terms of its structural contribution, but a substantive word refers to the meaning of the word in which it refers to the concrete

\footnotetext{
${ }^{36}$ The numbers beside the Pinyin hereafter expresses the tone the word takes with 1 meaning the first tone, 2 , the second tone, and so on.
} 
reality.) Zhou Boqi, a Yuan scholar further pointed that "jin1 zhil xu1 zi4 jie1 gu3 zhi1 shi2 zi4" (present-day grammatical words were all substantive words in ancient times.) (Sun, 1996, p. 11; see also Zheng \& Mai, 1964). A similar assertion was found in Givón's (1971, p. 413) “Today's morphology is yesterday’s syntax” (see also Heine et al., 1991, p. 12).

Grammaticalization is largely argued about in historical linguistics as a diachronic change phenomenon of open-class words to closed-class words. It is generally thought of as a process in which a lexical item becomes a grammatical or functional item, or a grammatical item becomes more grammatical (Hopper \& Traugott, 1993; Lehmann, 2002). For example, "have" with the lexical meaning of possession gradually takes on the grammatical function of completion, becoming the aspect marker of action fulfilment in "Have done". So grammaticalization is in fact a dynamic process in which the semantic elements of a lexical item gradually fade away but simultaneously it takes up more and more grammatical elements and even finally it has only grammatical functions and status. For example, "must" developed from the lexical word "mote" till finally grammaticalized as a modal auxiliary. Grammaticalization involves both diachronic and synchronic variants, though the diachronic aspect is the dominant. That is, in the grammaticalization process, there is also the situation or period where the items reflect various stages of grammaticalization and non-grammaticalized use at the same time, that is to say, they co-occur, e.g. "have" as a verb and as an aspect marker in current English. See e.g. Fischer et al. (2000), Fischer et al. (2004), Hopper \& Traugott (1993). But grammaticalization does not only apply to words, it also applies to structures or constructions. Grammaticalization is thus "a diachronic change by which the parts of a constructional schema come to have stronger internal dependencies" (Haspelmath, 2004, p. 26); specifically, it is "the change whereby in certain linguistic contexts speakers use parts of a construction with a grammatical function. Over time the resulting grammatical item may become more grammatical by acquiring more grammatical functions and expanding its host-classes" (Brinton \& Traugott, 2005, p. 99).

The literature on grammaticalization therefore suggests that grammaticalization is characterized by a gradual diachronic process with unidirectionality, i.e. from lexical 
to grammatical, from less grammatical to more grammatical, but not the other way around. The literature also regards this unidirectionality as consistent with the cognitive development, i.e. from the concrete to the abstract, from the more specific to the more general, and applies on all levels: Semantically, from fully referential to grammatical meaning, less subjective to more subjective; syntactically, from lexical to grammatical, less bound to more bound; phonologically, from full phonological form to reduced phonological form; pragmatically, from content meaning to pragmatic marker, propositional to attitudinal. So the widely held cline of grammaticalization would be:

Content item > grammatical word > clitic > inflectional affix (Hopper \& Traugott, 1993, p. 7)

This kind of development is considered to be motivated by some mechanisms, e.g. reanalysis and analogy. Reanalysis results in the cognitive process of metonymy and analogy brings in the cognitive process of metaphor (see Hopper \& Traugott, 1993, ch. $3 \& 4$ ). Probably due to the recognition of metaphor and metonymy as semantic change mechanisms in historical linguistics, they are also considered as processes underlying grammaticalization. For the most part, grammaticalization was viewed as a mere linguistic phenomenon in general, and as syntactic in particular, owing to the formal features of lexical items in syntactic structure. With grammar viewed from the cognitive perspective, e.g. Langacker (1987), and metonymy and metaphor as cognitive processes, e.g. Lakoff (1987a, 1987b), Lakoff \& Johnson (1980), and Lakoff \& Turner (1989), grammaticalization tended to be considered from the conceptual framework, e.g. Heine et al. (1991a), and metaphor and metonymy are regarded as the underlying cognitive processes as well, e.g. Heine et al. (1991a) and Hopper \& Traugott (1993).

\subsection{Grammaticalization: From concrete to abstract?}

From the preceding section it is obvious that grammaticalization, as widely held, is a process from semantic weight to grammatical weight, a process from losing semantic content to gaining more grammatical functions. This process of content words becoming functional words is regarded as from the concrete to the abstract, which is evidenced in arguments and debates around the unidirectionality feature of 
grammaticalization (see e.g. Olga Fischer et al., 2004; Olga Fischer et al., 2000; Haspelmath, 2004; López-Couso \& Seoane, 2008; Seoane \& López-Couso, 2008; Verhoeven et al., 2008; Wisher \& Diewald, 2002).

This appears to be somewhat at odds with the recent idea of cognitive science described in the preceding chapters that concept formation and concept development actually proceed from general and global to specific and local. And recent research results of cognitive psychology and developmental psychology have evidenced the global/general-to-specific development of both concept and category since infancy and throughout the life, see e.g. Behl-Chadha (1996), Mandler (1993, 1998b, 2004a, 2008) Nelson (2000), , Qinn \& Eimas (1998), Quinn \& Johnson (2000), and Younger \& Fearing (2000). As regards this inconsistency, it might be better to reconsider grammaticalization in the conceptual perspective. It has to be seriously rethought whether grammaticalization is conceptually from concrete to abstract.

It might be because semantic elements are considered as concrete and grammatical functions are considered as abstract that grammaticalization is regarded as a process from the concrete to the abstract. Grammaticalization is therefore a phenomenon at the interface of semantics and syntax in the traditional sense. But from the conceptual perspective, it would rather be that grammaticalization merges semantics and syntax (grammar) and blurs the traditional distinction between semantics and syntax. Thus, grammaticalization is right in the field of Cognitive Linguistics, and can be studied in the light of recent cognitive science theories, e.g. Embodied Cognition Theory, the above mentioned theory of global-to-specific conceptual formation and development.

Cognitive Linguistics views language as an integral part of cognition that reflects the interaction of cultural, psychological, communicative, and functional considerations, and holds that language can only be understood in the context of a realistic view of conceptualization and cognitive processing, and that any theoretical conception of language must be compatible with what is known about neurological organization and function. ${ }^{37}$ In Cognitive Linguistics, as introduced in Chapter 1, grammar is conceptualization (Croft \& Cruse, 2004; Vyvyan Evans \& Green, 2006; Langacker,

\footnotetext{
${ }^{37}$ See ICLA (International Cognitive Linguistics Association) website: http://www.cognitivelinguistics.org/aims.shtml
} 
1987; 1991, 1999), linguistic meaning is encyclopaedic and perspectival in nature and based on usage and experience (Evan et al., 2007a; Geeraerts, 1997; Geeraerts \& Cuyckens, 2007a). Cognitive Linguistics does not distinguish between semantics, syntax and pragmatics, for conceptual representation is the outcome of the nature of the bodies humans have and how they interact with the socio-physical world. Meaning, as it emerges from language use, is a function of the activation of conceptual knowledge structures as guided by context (Vyvyan Evans, 2010a). In this embodied sense, the semantic content of a word and its grammatical properties or functions originate from its use in human communication and are integral parts of the word as a whole conceptual entity. That is, in cognition, a lexical word (i.e. content word), when taken as a concept or a category, consists of its so-called semantic meanings, grammatical functions or properties, and pragmatic features in language use. All these are integrated together into the label form as a whole conceptual entity, i.e. the word. This is how Embodied Cognition goes and why Cognitive Linguistics stresses the encyclopaedic nature of meaning. A basic conceptual entity, e.g. a word, is comprised of various elements; its use for different goals and different functions determines the status of its components in the development. Even if for some purpose, one element fades to the background and thus pushes the other elements to the dominant status, e.g. from lexical (semantic) to grammatical, it is still PART TO PART within the conceptual entity. Therefore, in specific contexts, it is the perspectivization pointed out in Cognitive Linguistics that makes one part more salient than the other, and moves one part to the figure position and the other to the background. In this respect, grammaticalization is metonymic in nature, which is consistent with Langacker's (2004) idea that grammar is in nature metonymic ${ }^{38}$; it might proceed from the general to the specific (i.e. from WHOLE to PART) as well as from specific to specific (i.e. from PART to PART).

\footnotetext{
38 There are generally two views of grammar. One is in the narrow sense, i.e. grammar consists of syntax and morphology. The other is in the broad sense, i.e. grammar is the theory of language, which is widely held in Cognitive Linguistics, e.g. Cognitive Grammar, Construction Grammar. The idea that grammar is metonymic in nature was presented by Langacker (e.g. 2004). Grammar is regarded as essentially metonymic in that "the information explicitly coded linguistically does not itself establish the precise connections apprehended by the speaker and hearer in using an expression";"Explicit indications evoke conceptions which merely provide mental access to elements with the potential to be connected in specific ways - the details have to be established from other." In other words, the indeterminacy of language determines that language is only a clue to what it is intended to mean, it acts as a reference point providing mental access to others, implicit or explicit.
} 
Although Embodied Cognition originates from perceptual and sensorimotor activities and experience, perception cannot directly become conception. Percepts advance to concepts through perceptual meaning analysis ${ }^{39}---$ an innate cognitive process, which attentively re-interprets or re-describes or recodes what is perceived in a meaningful way to the perceiver in the form of image schemas (see e.g. Mandler, 1992, 1994, 1997, 2000, 2004a, 2005, 2007b, 2008; Mandler \& McDonough, 1993). Image schemas are the basic patterns of sensory-motor experience of embodied interaction with the environment; they play a crucial role in the emergence of meaning and in the performance of inferences and reasoning, and typically operate without conscious awareness (Johnson, 2005). Image schemas are insightfully advocated and fully employed to successfully explain linguistic structures and uses from the conceptual perspective in Cognitive Linguistics, and are experimentally proved to have cognitive psychological reality and neural grounding (see e.g. Clausner, 2005; Dodge \& Lakoff, 2005; Gibbs, 2005; Gibbs, 2006; Gibbs \& Colston, 1995; Hampe, 2005a; Johnson, 2005; Oakley, 2007; Rohrer, 2005). Based on the spatial structure of objects and their movements, image schemas represent the general patterns of spatial relationship of objects in embodied interactions such as PATH, UP-DOWN, FORCE, PART-WHOLE, GOAL, and CONTAINMENT; the image schema is a sort of holistic skeleton of spatial relations (see e.g. Hampe, 2005b; Johnson, 1987, 2007; Lakoff, 1987b; Lakoff \& Johnson, 1999; Lakoff \& Turner, 1989; Langacker, 1987). Image schemas are therefore primitive concepts or rather, conceptual primitives derived from perceptual meaning analysis of perceptual and motor processes, and are rather general or global in character (Mandler, 1992). Those global conceptual primitives gradually become specific and enriched with experience increment and language acquisition. Even with language acquired and language use, image schemas are still formed in cognition and kept in memory for future specification and enrichment whenever the situation creates the need. Thus the cognitive research on the formation and development of concepts, categories, image schemas and the crucial contributions they make to cognition and cognitive development, demonstrates that conceptual development proceeds from

\footnotetext{
${ }^{39}$ Although perceptual meaning analysis as a cognitive process is innate, the result obtained from the analysis might be subjective and different, because different subjects may have different perspectives even on the same object, movement, action, agent, and event. Therefore, with language acquisition and development, speakers with different languages might have different concepts and image schemas, even when they process the same situation through perceptual meaning process.
} 
global/general to specific. Grammaticalization, if taken as a process from the conceptual perspective, would probably still follow the global/general- to- specific development.

As mentioned in 5.1, grammaticalization went to the conceptual perspective in Heine et al. (1991a), and was also interpreted in terms of image schemas in the analysis of auxiliaries in Heine (1993) and possession in Heine (1997a). These research employed source structure and target structure in the grammaticalization process. The source structures are cognitive structures such as concepts composed of concrete objects, processes, or locations, and propositions expressing basic states or processes of human experience. The target structures are those grammaticalized outputs. Heine (1997a) adopted the term "schema" rather than "image schema" and extended the source structures to "the source schemas". In his analysis of possession, Heine used event schemas as the conceptual templates. For instance, in "a car belongs to me", the image schema consists of at least LOCATION (the car is located close to me), ACTION (I drive the car), COMPANION (I am accompanied by the car), GOAL (it is there for me), and EXISTENCE (it exists). In the source-target pattern, the target is the meanings the source schemas acquire through the grammaticalization process. Thus grammaticalization is a process from the source (concrete) to the target (abstract), and metaphor is thus thought to be the underlying mechanism of the source-to-target transition in which abstract concepts are understood in terms of less abstract concepts. It indicates a discrete transfer from one domain to another domain. Heine and colleagues also found another transfer, i.e. a gradual continuous transfer from a less to a more grammatical meaning, which they view as metonymy. In line with the metonymy-metaphor continuum in Cognitive Linguistics, this conceptual perspective of grammaticalization also takes metonymy and metaphor as a continuum of the process; i.e. rather than mutually exclusive, metonymy and metaphor are complementary aspects of grammaticalization. Although metonymy is acknowledged in grammaticalization, metaphor was suggested to be the more significant in Heine et al. (1991a), which seemed different from Hopper \& Traugott (1993) and Traugott \& König (1991). For example, grammaticalization was interpreted as the result of a problem-resolving process whose primary function is conceptualization by expressing one thing in terms of another, i.e. metaphoric (Heine et al., 1991b). However, metonymy is viewed as the more crucial mechanism 
in grammaticalization in Hopper \& Traugott (1993), which claims that even some instances of grammaticalization that have been regarded as metaphorical can be seen to arise out of contiguity, i.e. out of metonymy. This later idea is in line with the views this research holds and argues for.

As argued earlier, from the cognitive perspective, metonymy as a natural cognitive mechanism underlies grammaticalization and is embedded in the global/general- tospecific process and the PART-PART relation between semantics and syntax. It can be illustrated that grammaticalization abides by the global/general-to-specific conceptual development. Take "go" as the first example. It is argued in the grammaticalization literature that "go" develops from a lexical action verb to a functional auxiliary expressing future tense as in be going to. Motion events are basically first conceived and conceptualized in image schemas of AGENCY and SOURCE-PATH-GOAL (Johnson, 2005, 2007; Lakoff, 1987b; Mandler, 2004a, 2006), not specific actions. The first concepts of objects and artefacts tend to be general and global and organized around paths of motion (Mandler, 1992, 2004a, 2006). As a motion verb, go is goal-oriented with path changing, agency moving, and source leaving, and all these occur in the perception of the motion and are recoded into an image schema of GO through the perceptual meaning analysis mechanism. This general concept of go contains a lot to be specified. Goal-oriented means a purpose is carried on, thus go can be used from goal-oriented movement to intended action; changing path means constant changing state of the agency, thus the intended action can be used to express a predicted state, i.e. the future tense. Of course, it is the context that go is used in that triggers the grammaticalization of $g o$ to a functional auxiliary of the future tense. Hopper \& Traugott (1993) specifically mention the role to played in the grammaticalization of go to express the future tense. It is right because of to (its purposive meaning), which expresses GOAL of the image schema GO, that be going to can be grammaticalized to a future-tense auxiliary (cf. Ungerer \& Schmid, 2006, pp. 321-326). Grammaticalization of go is actually the grammaticalization of the construction be going to, i.e. it is the development of this construction in use that results in the future-tense feature of be going to. The conceptualization of motion is rather general and global in terms of image schema. This generality is to be specified in actual use. 
Another example is from Chinese. You3 in the construction of you $3+$ verb (phrase) in Min and Yue dialects is said to be an adverb, i.e. you3 is grammaticalized from a lexical verb to a pragmatic adverb (Shi, 2006; Shi \& Li, 2004). If so, it is also a process from general and global to specific. A diachronic corpus survey of you 3 in ancient and modern Chinese literature shows that you3 was used rather generally, involving the general concepts of "possession" and later, "existence" with varied word classes (Fu, 2006, 2007). The grammaticalization of you3 is a developmental process from the primitive prototype of POSSESSION, which contains/presupposes the knowledge of some existence (i.e. something exists), to the specific prototype of EXISTENCE $^{40}$, and then to the pragmatic marker indicating some action or event has already happened, nominalizing the following $v p$ which in turn weakens the motion property of you3 (Fu, 2006).

It seems that, from the conceptual perspective, grammaticalization is a process with the following characteristics: (1) Movement from general and global to specific, (2) it undergoes a metonymic process. Since verbs are used to express function conceptually (Ahrens, 2002, 2010), function is indispensible to verb category. That function comes to the fore in development is obviously motivated by the metonymic process; the process of grammaticalization hence appears to be on the side of the general/global- to- specific theory of cognition.

\subsection{Metonymy in grammaticalization}

As already pointed out in the foregoing sections, metonymy has been acknowledged as one of the significant mechanisms in grammaticalization in the literature. Nevertheless, it is argued here that in the conceptual perspective grammaticalization as a process tends to go from the general and global to the specific, and grammaticalization is cognitively motivated principally by metonymy when considering the relation of metonymy and metaphor discussed in the preceding chapters. In grammaticalization, it is probable that metonymy as a natural cognitive mechanism enables the grammatical element of a word to stand up as the figure and the semantic element to move backward to the ground within the word as a

40 As for meaning prototype, please see the succeeding chapter. 
conceptual entity, and it is likely that metonymy licenses the process of grammticalization from general and global to concrete and specific.

Metonymy is in charge of forming a whole conceptual entity such as concept, category, image schema, event schema, as well as relating PART to PART and PART to the WHOLE within the formed whole pattern. Even the widely held interdomain relating mechanism metaphor has to presuppose the PART-WHOLE relating before inter-domain mapping can take place, though it is not meant to ignore a metaphoric role in grammaticalization. However, metonymy seems to be more essential than metaphor in grammaticalization. This section will discuss how metonymy functions in grammaticalization through analysis of grammaticalization cases in both Chinese and English.

\subsubsection{Chinese Case: Hao3dai3 (good and bad)}

Hao3dai3 in Chinese is an antonymous compound consisting of two adjective words whose lexical meanings are opposite (hao3: Good; dai3: Bad). It currently functions as both a noun, and an adverb (both temporal and modal) in Chinese in terms of grammatical property, and is argued to have undergone grammaticalization from adjective to noun, from adjective to adverb, and from notional words to functional words. It becomes a compound noun because: (1) The lexical meaning of hao3 is gradually lost in use and thus a unilateral compound noun is formed, and (2) it is motivated by metonymy (adjectives hao3 and dai3 are used to stand for results, i.e. THE NATURE OF RESULTS FOR THE RESULTS THEMSELVES); it becomes a modal adverb because of metonymy (two opposite poles to mean whatever, anyhow, or anyway, i.e. TWO RESULTS FOR ANY RESULT); it becomes a temporal adverb because of metaphoric motivation (a mapping is assumed to be projected to hao3dai3 from other antonymous compounds such as zao3wan3 (early and late), duolshao3 (more and less), da4xiao3 (big and small), and gaoldil (high and low) in light of iconicity, i.e. similar form indicates similar meaning) (Fang \& Zeng, 2007b).

While approval is given to Fang \& Zeng's metonymic analysis in hao3dai3's grammaticalization, the metaphoric motivation of hao3dai3 as a temporal adverb sees a divergence. Now let's analyse the grammaticalization process of this compound word one by one. First of all, hao3 and dai3 are independent adjectives; 
the initial combining use of hao3 and dai3 was in coordinate status in terms of meaning, indicating "hao3-dai3" (good and bad). For example ${ }^{41}$ :

(25) 做儿的不知好歹, 做娘的不辨清浊。

Zuo4 er de bu4 zhi1 hao3dai3, zuo4 niang2 de bu4 bian4 qing1 zhuo2

The son doesn't know which is good and which is bad; the mother doesn't distinguish the pure from the impure.

（26）地也，你不分好何为地？天也，你错勘贤愚枉做天！

Di4 ye3, ni3 bu4 fen1 hao3 dai3 he2 wei2 di4? Tian1 ye3, ni3 cuo4 kan1 xian2 yu2 wang3 zuo4 tian1

Earth, you are totally wrong since you cannot tell what is good from what is bad; Heaven, you are totally wrong since you cannot tell the wise from the silly.

In these two examples, hao3dai3 is a compound of two adjectives; however, it factually acts as a compound noun, expressing what is good and what is bad, i.e. the good and the bad, i.e. RESULTS FOR THE WHOLE EVENT/SITUATION. But with frequent use of this compound, its semantic weight moves to dai3 with the meaning of the bad result in general, the worst result in particular, thus becoming a unilateral compound. For example:

(27) 这会子你倘或有个好歹，丢下我，叫我靠那一个!

Zhe4 hui4 zi3 ni3 tang3 huo4 you3 ge4 hao3 dai3, diu1 xia4 wo3, jiao4 wo3 kao4 na3 yil ge4

If you drop off and leave me alone, no one would support me.

(28) 若孩儿有些好歹, 老身性命也便休了。

Ruo4 hai2 er you3 xie1 hao3 dai3, lao3 shen1 xing4 ming4 ye3 bian4 xiu1 le.

${ }^{41}$ The examples of hao3dai3 are all from Fang \& Zeng (2007b) with my own translations, except otherwise noted. Note, the translations cannot perfectly reproduce the original meaning and they are used here to give an idea of how hao3dai3 is actually used in terms of grammaticalization. 
If there is any mishap to the child, that would end my life too.

Hao3dai3 in the above examples means the worst result, i.e. death. So far there are two stages involved here. The first is hao3dai3 developed from adjectives to nouns in terms of syntactic feature and from "good" and "bad" to "something good" and "something bad", i.e. "the good" and "the bad", in terms of semantic value. The second hao3dai3 is thereby developed from a coordinate compound to a unilateral compound with the semantic value moving onto the latter dai3; thus the whole compound expresses the meaning indicated by dai3 only. As noted in the analysis, these two stages are both motivated by metonymy, which is not made clear in Fang $\&$ Zeng (2007b). In the first stage, the evaluative feature of something is used for the thing: Good for the good, bad for the bad, it might be termed as PART FOR WHOLE. In the second stage, "the good and the bad" is used for "the bad" only, i.e. WHOLE FOR PART. Of course, this kind of semantic value inclination to "the bad" only is related to the negative context in the use of this compound. As Cognitive Linguistics announces, meaning is constructed in language use, which originates from embodied interactions. A similar idea is also pointed out by Grammaticalization scholars, e.g. Heine et al (1991a, p. 27) say, “...grammaticalization itself is motivated by extralinguistic factors, above all by cognition."

There is another situation analysed in Fang \& Zeng (2007b) regarding hao3dai3 as a compound noun. Hao3dai3 in this situation is different from the first stage mentioned above in that hao3dai3 is not a simple coordination of hao3 (good) and dai3 (bad), it instead metonymically expresses the result; different from the second stage in that hao3dai3 has no semantic weight movement. That is, hao3dai3 is used to express RESULT, be it good or bad. This process is obviously metonymic, the general two evaluative adjectives hao3 and dai3 are used to express the overall result with no weight on good or on bad. So it can be termed PART FOR WHOLE metonymy, in which the two resultative situations altogether represent the overall result. For example:

（29）老孙还要打开那门，与他见个好歹，恐师父在此疑虑盼望，故先来 回个信息。 
Lao3 sun1 hai2 yao4 da3 kai1 na4 men2, yu3 ta1 jian4 ge4 hao3 dai3, kong3 shi1 fu zai4 ci3 yi2 lv4 pan4 wang4, gu4 xian1 lai2 hui2 ge xin4 xi

Lao Sun was trying to open the door to have a good fight with him, but considering that Shifu would be worried about us, I came back to inform you of what was happening.

In examples of this kind, hao3dai3 means a result, an end. Thus in this case the compound uses two opposite results (hao3 and dai3) to represent any result, i.e. the overall result. Therefore from adjectives to acting as nouns in the compound, from "good" and "bad" to "the good" and "the bad", to "the bad" only (the worst result), and to result in general, the antonymous compound hao3dai3 undergoes metonymic transfer, which actually also involves a natural process from "the good" and "the bad" to "the good result" and "the bad result" before reaching "the bad result" only, i.e. from general to specific, or in the general terms WHOLE FOR PART.

Hao3dai3 as a compound of antonymous adjectives acted as an adverb as well in the development process of this compound. It functions as both a temporal and a modal adverb. As mentioned earlier at the outset of this section, Fang \& Zeng (2007b) think hao3dai3 developed to a temporal adverb through metaphorical mapping, based on the similar structure as other antonymous compounds such as duolshao3, da4xiao3 and gaoldil. Instead it is argued here that the development of hao3dai3 into a temporal adverb is instead motivated by metonymy. Let's first see some examples:

(30) 兄弟, 拿他做甚么? 他吃了酒好去也。

Xiong1 di4, na2 ta1 zuo4 shen2 me? Ta1 chi1 le jiu3 hao3 dai3 qu4 ye3

Buddy, why not stop doing anything to him? He will leave sooner or later after the wine.

(31) 如今那老婆子害病, 我讨服药与他吃了, 药死那老婆子, 这小妮子 好歹做我的老婆。

Ru2 jin1 na4 lao3 po2 zi3 hai4 bing4, wo3 tao3 fu1 yao4 yu3 ta1 chi1 le, yao4 si3 na4 lao3 po2 zi3, zhe4 xiao3 ni1 zi3 hao3 dai3 zuo4 wo3 de lao3 po2 
Now the old lady is ill, I can get wrong medicine for her and poison her to death, and then the girl will be my wife sooner or later.

In fact the temporal adverb interpretation or analysis is determined by the context, which leads to the time aspect telling when something happened or has happened or will happen. It is directly related to the global sense of hao3dai3, i.e. the good and the bad. Anything can be recognized, or rather, evaluated as good or bad in terms of result, the globality of this sense licenses hao3dai3's word class development and meaning change. In example 30, hao3dai3 is used after tal chil le3 jiu3, literally indicating what will happen after an incident, i.e. after the wine he will leave for better or worse (but literally in Chinese it means whether it is a good or a bad result). In example 31 , hao3dai3 is also used after an incident, indicating what will happen after that, i.e. after the old lady dies, the girl will become my wife whether it is a good or a bad result. But metonymically it means the ending time "sooner or later" in terms of temporal development. The result is what is obtained at the ending time. Therefore, although interpreted as a temporal adverb, hao3dai3 still emphasises the resultative situation, i.e. what result it will be after some incident. That is also to say that this kind of development is still not beyond the evaluative and resultative base. It is clear that this development is not through metaphor as declared by Fang \& Zeng (2007b), but through metonymy, i.e. from the whole to the part. Results indicate the final ends, thus hao3dai3 also developed to mean "finally", "at last", for example:

(32) 好歹找着了。(from Baidu search engine)

Hao3 dai3 zhao3 zhao2 le

Finally it was found.

To put it simply, hao3dai3, as a compound of two evaluative adjectives of results, keeps its defining evaluative and resultative properties; its development into a temporal adverb compound derives from the focus on its resultative nature, and its development into a modal adverb compound derives from the focus on its evaluative nature, as shown in the following examples:

(33) 这毒药在谁家合来? 这服药好有个着落。 
Zhe4 du2 yao4 zai4 shui2 jia1 he2 lai2? Zhe4 fu2 yao4 hao3 dai3 you3 ge4 zhuo2 luo4

Where did you get this poison? Anyway, it does not come from nowhere.

(34) 我不会唱。你好互唱一个曲儿, 我吃不的闷酒。

Ni3 hao3 dai3 chang4 yi1 ge4 qu3 er, wo3 chi1 bu2 de men1 jiu3

I don't know how to sing. Anyhow, you can sing a song; I cannot drink without listening to songs.

(35) 闲语休说, 好歹要房宿饭钱还我。

Xian2 yu3 xiu1 shuo1, hao3 dai3 yao4 fang2 su4 fan4 qian2 huan2 wo3

Stop chit-chatting, after all you need to pay me for the lodging and dining.

This modal adverbial use of hao3dai3 also originates from hao3 and dai3 - the two semantic poles of the evaluative result continuum. These two poles are used to represent the whole continuum, i.e. through PART FOR WHOLE metonymy, indicating the overall result, i.e. either bad or good. Thus it is easily used together with resultative actions or incidents in the context to mean the modal sense such as "whatever", "anyway", "anyhow", "in any case/event”, and "after all”.

From the above analysis, it is clear that hao3dai3, as a compound comprised of two resultative-evaluative adjectives, developed to a compound noun, and to a compound adverb in terms of syntactic function; developed from "good" and "bad" to mean "the good and/or the bad", "the bad/worst result", "sooner or later/after all", and "whatever/anyhow/anyway" in terms of semantic function, and this process appears to be metonymically motivated. From the conceptual perspective, this metonymymotivated grammaticalization starts directly from the general/global image schema formed by hao3 and dai3, i.e. from the global evaluative and resultative concept, to the specific result, e.g. "the good and/or the bad", "the bad", "the worst", "any specific result, either good or bad". The image schemas of hao3 (good) and dai3 (bad) might originate from perceptual meaning analysis of interactions of objects in 
spatial movement with the body, e.g. occlusion, containment, collision mentioned in section 3.2.4, Chapter 3. Hao3 and dai3 are two words for describing two specific situations of the resultative-evaluative image schema, and they are two prominent aspects of the global image schema, they cannot exist without each other. Their combination is psychologically permitted and conceptually licensed by the resultative-evaluative image schema, for the "core meanings" of grammatical forms are likely to be caught in their youngest uses (Slobin, 1994, p. 120), and even preschool-age children can easily reach the core semantic concepts and pragmatic functions without as much experience as adults (Slobin, 1994, p. 129). This global/general-to-specific theory and cognitive analysis also applies to other Chinese antonymous compounds such as duolshao3 (more and less), gaoldil (high and low), da4xiao3 (big and small), zheng4fan3 (positive and negative), qian2hou4 (fore and after), zuo3you4 (left and right), li3wai4 (inside and outside), etc. Conceptually hao3dai3 proceeds in general from the global image schema to the specific with the development of situations, as analysed above. Although there are some PART FOR WHOLE operations in the grammaticalization of hao3dai3, these are not PARTWHOLE FORMING, but PART-WHOLE RELATING, since the whole has already formed and is inflicting invisible impact upon conceptual perspectivization and specification in cognitive activities; as argued in the section about infants making inductive inference in Chapter 3, this kind of impact is most of the time beyond conscious awareness.

That grammaticalization goes from the global/general to the specific through metonymic operation can also be illustrated in another Chinese case di 3(bottom). According to Yap et al. (2010), the etymology of di 3 底 (bottom) can be traced to an ancient lexical noun di3 氏, which was often used in the sense of "root" or “foundation". However, with di3 氏 gradually encompassing many other meanings in frequent use, semantic radicals were added to form new characters with more specialized interpretation. For example, with the semantic radical mu4 木 (wood), comes di3 柢 with the meaning of root; with the semantic radical ren2人 (man), comes di 1 低 with the meaning of low or to lower, i.e. people in low class; with the abstract semantic radical guang3 广 (wide, broad, vast), comes di3 底 with the meaning of bottom (of any entity). Nevertheless, the semantic radical guang3 广 
(wide, broad, vast) is fairly general in sense, encompassing everything, everywhere, from spatial to temporal, to other categories, although it was originally created with a spatial reference (this is the primary character of Chinese characters) ${ }^{42}$. Thus with this image schema of guang 3 广 as the semantic radical, di3 底 can be extended to mean the bottom (part) of any entity (object, event, action, process, etc.). This global feature of di3 底 actually paves the way for its grammaticalization from a locative noun to a light noun and further to an interrogative pronoun and an attitudinal nominaliser. See Yap et al. (2010) for detail.

Specific meanings, or rather, meaning specifications of a word are part of the word as a concept, meaning specifications of a construction are also part of the construction as a category; probably they are all specifications of the overall image schema formed through metonymy at the early stage of cognitive development. Grammaticalization, taken as a conceptual development, inevitably follows suit. In general it goes from the global/general to the specific, seemingly motivated by metonymy all the way. In light of the metonymic global-to-specific theory of Embodied Cognition, a second and careful unbiased analysis of those grammaticalization cases formerly interpreted as motivated by metaphor will discover that metonymy underlies the process and directs the way from global/general to specific, which can be evidenced by more Chinese cases such as duolshao3 (more and less) (Fang \& Zeng, 2007a), Chinese locative construction (Sun, 2008), ba3, le, and lai2 (Sun, 1996), gei3 (Ting \& Chang, 2004), and guo4 (Iljic, 2009).

\subsubsection{English case: Auxiliation}

Auxiliation is the process of "complex lexical verb structures developing over time into auxiliary grammatical structures, with all its accompanying semantic, morphosyntactic, and phonological changes" (Kuteva, 2004, p. 2). Auxiliation results in auxiliaries, which "express grammatical concepts typically relating to the temporal state (tense), the temporal contours (aspect), and the type of reality (modality) of propositional contents" (Heine, 1993, p. 28). This process of

\footnotetext{
${ }^{42}$ Although there are some pictographic characters in Chinese, Chinese characters are essentially metonymy-motivated. In the six categories of Chinese Characters, except pictographic, all other categories, i.e. ideographic, self-explanatory, semasiographic and mutually explanatory, are motivated by metonymy.
} 
auxiliation is thus typically one of grammaticalization: Lexical verb structures developing into functional structures expressing tense, aspect and modality. It is also considered to develop from concrete to abstract in the literature (see e.g. Heine, 1993; Kuteva, 2004).

However, in light of Heine's own Source-Target pattern, the source concepts for auxiliation are not specific verbs, i.e. verbs expressing specific motions, manners of movement, e.g. "walk" and "swim", but general verbs, i.e. verbs which lack specifics regarding the nature of the movement, e.g. "come" and "go". As mentioned by Heine (1993, pp. 28-29), these verbs become the sources for auxiliation because they are general in sense and can be used in a much wider range of contexts with high frequency, and the high degree of generality enables these verbs to appear in constructions entering grammaticalization in general, and auxiliation in particular. This general- to- specific tendency of grammaticalization (including auxiliation) was in fact also pointed out by scholars in the field of grammaticalization. For example, according to Heine (1993, p. 29), Kuteva (1991) observes that most of the verbs acting as the source for auxiliation are characterized by a high level of generality; it is concepts like "walk" and "run", but more general concepts like "go" and "come", that license auxiliation, because these general concepts encode a limited number of kinaesthetic image schemas such as SOURCE-PATH-GOAL, CONTACT, PARTWHOLE, etc. By the same token, Mkhatshwa (1991) argues that it is the generic members of the verb category that tend to be grammaticalized to auxiliaries. In his analysis of Zulu verbs with the features "motion" and "direction", which consists of verbs such as -za "come", -ya "go", -ngena "come in", -sondela "come near", and dilika "come down", Mkhatshwa finds that only -za "come" and -ya "go" are grammaticalized as tense markers "-za" as a marker of immediate future and -ya as a marker of remote future tense; he argues that this is decided by the degree of generality of these two verbs: They are semantically the least constrained, they exhibit the widest scope of usage, and may in certain contexts substitute for other members of the domain but not vice versa (see Heine, 1993, p. 28).

Even though some scholars have realized this issue, the concrete- to- abstract development is unfortunately still widely held in grammaticalization research. But as pointed out earlier, this view seems problematic. In addition, the widely held 
unidirectional concrete- to- abstract development of grammaticalization cannot interpret the grammaticalization cases through specification, e.g. auxiliation. Kuteva (2004, pp. 35-42) argues that auxiliation development is not always crosslinguistically along the concrete- to- abstract continuum, there are cases of auxiliation and grammaticalization motivated by a specification mechanism whereby an additional semantic element is added to the structure that already has a distinct and initial semantic content. However, these cases can be well analysed and interpreted by the general/global-to-specific principle of cognition. The specification mechanism termed by Kuteva is metonymical in nature; it is actually a case where one specific part gains salience while other parts fade back, i.e. it is still a WHOLEPART metonymy. One of the examples cited in Kuteva (2004, p. 37) is the Alicante dialect of Spanish quoted from Schwenter (1994), in which the present perfect form (signalling that the situation occurs prior to the reference time and is related to the situation at reference time) has taken on a new grammatical function, i.e. that of the hodiernal past, which is used to express same-day events regardless of their current relevance:

(36) EL papá ha trabajado ûallí. (Alicante Spanish)

The dada have ( 3 singular, i.e. use the third person singular of have) worked there

'Dad has worked there,' (so he can tell us about the sites' working conditions.)

While the present perfect tense indicates that something has taken place and has a relation to the reference time, the mere mention of the occurred event regardless of its current relevance is only part of the whole story; the focus is moved from the occurrence and the current relevance to the occurrence only. This suggests that it is metonymy that motivates this specification, in Kuteva's term. It is still from the general/global to the specific.

In the Source-Target structure analysis of grammaticalization (Heine et al., 1991) and auxiliation (Heine, 1993) from the conceptual perspective, image schemas, and event schemas, to be specific, are employed to cognitively interpret those phenomena. Such event schemas as LOCATION, MOTION, ACTION, VOLITION, CHANGE-OF-STATE, EQUATION, ACCOMPANIMENT, POSSESSION, and 
MANNER are supposed to be the sources for the targets (i.e. grammaticalized items). Among those event schemas, the LOCATION, the MOTION, and the ACTION schemas are considered to be the more basic and constitute more salient event schemas of human conceptualization. These basic, or rather, these few general event schemas are specified in a particular linguistic context of use and result in other more specific event schemas. In fact, of these three event schemas, LOCATION is a specified event schema in MOTION and ACTION schemas. With MOTION and ACTION schemas considered together and ACTION put into the broad sense, it is likely to subsume MOTION into the ACTION schema, taking MOTION as a specified schema of the ACTION schema, since ACTION always suggests a certain change of position of some sort (either concrete or abstract). Therefore, it is reasonable in cognition that the ACTION schema is the essential source schema, and all other event schemas are specification or perspectivization of the ACTION schema. This is in line with Embodied Cognition Theory that action organizes human conceptual activities. And this general/global- to- specific process is inevitably overseen by metonymy.

Now let's see a typical example of auxiliation - the future tense marker be going to. Hopper \& Traugott $(1993 ; 2003)$ analyse be going to with the help of syntagmatic and paradigmatic axes, i.e. reanalysis and analogy, relating these two to metonymy and metaphor respectively. For instance:

\begin{tabular}{|c|c|c|}
\hline $\begin{array}{l}\mathbf{B e} \\
\text { progressive }\end{array}$ & $\begin{array}{l}\text { going } \\
\text { directional verb }\end{array}$ & $\begin{array}{c}\text { [to visit Bill] } \\
\text { [purposive clause] }\end{array}$ \\
\hline [be going to] & visit Bill & \\
\hline Tense & action verb & (by metonymy) \\
\hline $\begin{array}{l}\text { [be going to] } \\
\text { Tense }\end{array}$ & like Bill & (by metanhor) \\
\hline
\end{tabular}

In Hopper \& Traugott's eyes, it is because the analogical replacement of "visit" by "like" that extends be going to to the full future tense marker; and they thus consider that this process is enabled by metaphor (Hopper \& Traugott, 1993, pp. 87-88). However, a second analysis will find that this so-called analogy is embodied by "VERB FOR ACTION VERB", i.e. it actually relates a PART to the WHOLE. That 
is to say, this process is a PART-WHOLE RELATING process since the action verb is a component of verb in terms of category, which is obviously motivated by metonymy, not by metaphor, as demonstrated in Chapters 3 and 4 .

In terms of event schemas, be going to comes to the future tense through a MOTION schema. First of all, go as a motion verb schematically and conceptually involves at least SOURCE, PATH, GOAL, and AGENCY. This general schema indicates go has the potential to be used together with different words expressing goal/destination, intention (i.e. involving Heine's VOLITION schema), and prediction. The present progressive tense of $g o$ is destined to go with to plus a goal/destination, for example:

(37) Billy is going to Auckland. (Agency Billy + progressive go + directional to + destination Auckland)

(38) Billy is going to Auckland to find his sister. (Agency Billy + progressive go + directional to + destination Auckland + purposive to + goal find his sister)

In the above examples, the destination and the goal are specified and highlighted respectively in the MOTION schema of GO, i.e. the focus is moving within the MOTION schema by metonymy, from the general to the specific. Since the second example shifts focus to the goal, the destination part becomes less salient and fades to the background and can even be ignored, thus it is natural to have the following:

(39) Billy is going to find his sister. (Agency Billy + progressive go + purposive to + goal find his sister)

This focus movement, however, simultaneously suggests that intention is highlighted. That is to say, when GOAL is focused, it is natural that the intention of the agency is specified and highlighted. Thus in example (39), the goal-oriented movement starts to stand for intended action, and intention is prioritized. GOAL goes with INTENTION, and INTENTION can be said to be part of GOAL in an event schema. Because of this, here the progressive go and purposive to together as a construction is developing to the future tense marker. As mentioned by Ungerer \& Schmid (2006, p. 323), "the notion of purposeful or intended action comes with the expectation of future enactment, the verb form is going acquires an aspect of futurity in this 
construction." The process of goal-oriented actions/motions developing to intentionoriented actions/motions or intended actions/motions is metonymically motivated conceptually. This intention-focused action or motion together with the AGENCY will expect a certain result within the event schema in cognition. And the future tense is strengthened in a situation of this kind, which is illustrated in example 40:

(40) Billy is going to be a hero/like the experience. (Agency Billy + future tense be going to + verb phrase be a hero/like the experience)

Example (40) shows that the intention is moved to prediction in the light of the event schema, and be going to is totally grammaticalized into a future tense marker while the action verbs following the progressive construction change to any kind of verb in the event construction and the resulting prediction of the intended action becomes conceptually predominant through metonymy. Ungerer \& Schmid (2006, pp. 322324) argue that the development from the intended action to the predicted state is enabled by metaphor, i.e. PREDICTED STATE IS INTENDED ACTION. When this development from the perspective of the event schema of GO and the constructions containing "go", e.g. from directional "to" to purposive "to" and goal verb phrases, is taken into account, it is self-evident that in cognition the motion event schema of GO inherently has a goal orientation which permits a following purposive to that inherently has an intentional orientation (see e.g. Fischer, 2000; Haspelmath, 1989). This development proceeds from the general/global to the specific, from MOTION schema to its specification in terms of VOLITION schema; this process, as analysed above, is conceptually motivated all the way by metonymy, not by metaphor even at the last stage as argued by Hopper \& Traugott (1993/2003, p. 61, p. 88), Heine (1993, p. 96) and Ungerer \& Schmid (2006, p. 323).

In English, this general/global- to- specific development theory of cognition and the underlying cognitive mechanism metonymy can also be applied to analysis of other grammaticalization cases such as concession (Chen, 2000) and possession (Heine, 1997b). Nonetheless, more analysis of auxiliaries in English such as can and must and in other languages, together with more research on other grammaticalization phenomena in English and other languages is needed to examine to what extent metonymy operates in the grammaticalization process. 


\subsection{Summary}

Grammaticalization has long been researched in linguistics. With the emergence of Cognitive Linguistics and conceptual motivation analysis of linguistic structures and uses, grammaticalization is being examined from the conceptual perspective. It is especially interpreted and analysed in the light of image schemas in general and event schemas in particular. Nevertheless, grammaticalization is still commonly considered to be a process from the concrete to the abstract. Even though some scholars find out that grammaticalization, e.g. auxiliation (Kuteva, 2004), does not always follow the concrete-abstract unidirectionality, it seems that so far no attempt has been made to propose an argument from the other way round for a consistent theoretical rationale for grammaticalization phenomena.

Conceptually considered, grammaticalization blurs the distinction between semantics and grammar (syntax). Grammaticalization is at the interface of semantics and grammar (syntax). In other words, in grammaticalization, there is in principle no cognitive difference between semantics and grammar (syntax), which is in line with Cognitive Linguistic claims that semantics, syntax and pragmatics are organically bundled together, grammar and meaning construction are conceptualization, and semantic structure is conceptual structure. Recent cognitive science research suggests that concept and category develop from the global/general to the specific. Grammaticalization as a cognitive process seems to follow this development tendency in essence. The event schematic analysis of grammaticalization actually evidenced this point; e.g. the schematic analyses of possession (Heine, 1997b) and auxiliation (Heine, 1993; Kuteva, 2004) are pioneering attempts, although the concrete-abstract development is still maintained. The fact is that image schemas or event schemas are conceptual primitives, which are global and general in nature and gain specification and enrichment in development. These image schemas or event schemas as whole conceptual entities highlight different inherent component parts in actual language use and thus bring them to the focus position in cognition. This WHOLE-PART process is conceptually motivated by metonymy.

To support this point, grammaticalization cases in both Chinese and English have been analysed in the light of image schemas and event schemas. In Chinese, the 
antonymous compound hao3dai3 develops from coordinate adjectives hao 3 and dai3 to unilateral hao3dai3 with semantic weight on dai3, to the functional weight indicating "anyway", "whatever", "after all”, from compound adjective to compound noun, to adverb. Nevertheless, this process goes from the general to the specific. This grammaticalization procedure is enabled by the image schemas of hao3 and dai3. Conceptually hao3 and dai3 are both concepts expressing evaluation, which are inherently resultative. The inherent evaluative and resultative properties of hao3 and dai3 pave the way for the compound's grammaticalization. It is clear that this process develops overall from the general/global to the specific aspect within the cognitive schema, overseen by WHOLE-PART metonymy.

In English, the future tense marker be going to originates from the image schema of GO, which as a conceptual entity at least consists of PATH, GOAL, AGENCY, SOURCE. GO as a motion inevitably relates GOAL, PATH and AGENCY. Its GOAL aspect inherently relates to direction, purpose and intention (AGENCY also relates to this as well), thus naturally followed by both the directional to and purposive to. The intentional aspect of the GO schema relates to the result intended, thus this intended result might be predicted in the cognitive framework of intention. Therefore the progressive GO and purposive to together strengthen the futurity of be going to. It is clear that this grammaticalization is from the general to the specific and motivated by metonymy as well.

Grammaticalization can be viewed as a dynamic process from semantic weight to syntactic weight within the encyclopaedic knowledge of the word as a conceptual entity, and it operates at the interface of the traditional semantics and syntax. In this sense, it might be said that there is no semantic change in grammaticalization. What is happening in grammaticalization seems to be just figure-ground shift due to frequent exposure to a certain context. Grammaticalization is accordingly different from shifts in word meaning such as generalization, specification, amelioration, and pejoration. All these are shifts within the traditional semantic aspect, and are in essence irrelevant to the syntactic function of the word, though amelioration and pejoration tend to have some relation with the pragmatic aspect in communication. Nevertheless, as mentioned earlier in Chapters 1 and 2, Cognitive Linguistics is liable to make no distinction between semantics and pragmatics in principle. These 
semantic shifts could be taken as different from grammaticalization. But it could not be denied that grammaticalization might go hand in hand with the development of meaning prototype, i.e. grammaticalization might take place after a change or serial changes in the meaning prototype (for meaning prototype, please see the succeeding chapter).

In sum, the general/global-to-specific rationale and its inherent cognitive mechanism metonymy could be successfully employed to interpret and analyse grammaticalization in the cognitive framework. Although more cases in different languages need to be done to provide verification, the rationale and the mechanism as covered so far are viable. 


\section{Chapter 6 Metonymy and Meaning Prototype}

The preceding chapter examined the function of metonymy in grammaticalization. It contended that grammaticalization is actually a cognitive process which conceptually tends to go from the general/global to the specific rather than from the concrete to the abstract, and this process is motivated essentially by metonymy; and it also illustrated this point by conceptual analysis of hao3dai3 in Chinese and auxiliation in English in the light of image schemas.

This chapter will explore the role of metonymy in another aspect of language: Meaning, or meaning prototype, to be specific. Here meaning is considered from the cognitive perspective as a prototypical concept. From the polysemous viewpoint, this chapter will first examine various views of meaning such as meaning selection, meaning creation, meaning construction, meaning potential, and meaning organization such as the traditional view, the abstractist view, and the prototype view. The prototype view is widely held in Cognitive Linguistics. Despite the discussion of prototype in lexical and cognitive semantics as context dependent, changeable, and dynamic, some presentation seems to indicate a fixed rather than a dynamic and developmental prototypical meaning for a polysemous word.

The dynamic nature of prototype was mentioned by Geeraerts (1997). The dynamic in his view relies on the peripheral cases of the category, i.e. the dynamic of prototype is the result of the assumption that the peripheral nuances are changing and expanding around the kernel rather than determinate (the boundary is not clear cut). Therefore Geeraerts's view of the dynamic of prototype implies a fixed prototype centre and only the cases around the centre are dynamic. However in his analysis of word meaning, Geeraerts also finds two or more centres in the category; unfortunately he does not step a little further to give the real sense of dynamic to prototype.

Instead it will argue here that meaning as a prototypical concept is itself functional and developmental. Meaning develops in accordance with meaning prototype. The dynamic nature of prototype relies on the dynamic prototypical centre. Meaning prototype develops in the uses of the word. In a different period or scenario, different meaning prototype will come to the forefront as the figure and the previous meaning 
prototype thus fades back into the ground, which may be sometime ushered to the figure position again in a later scenario. This point will be illustrated through the analysis of "110" in China in a corpus-based approach.

\subsection{Meaning in the perspective of cognition}

Meaning, especially word meaning, has been the research focus of philosophy, psychology, linguistics, and artificial intelligence. Meaning is the real value of the existence of a word. The point that "language is all about meaning" is foundational (Geeraerts, 2006, p. 3). Cognitive Linguistics holds that the structure of language reflects the conceptual system of human mind (Vyvyan Evans \& Green, 2006; Geeraerts, 1997; Lakoff \& Johnson, 1980), but it is meaning that bridges and unites language and thought. Verbal thought takes word meaning as its unit, which reflects a generalized mental reality; meaning as an act of thought and an inalienable part of a word is in the realm of language as much as in the realm of thought (Vygotsky, 1986, pp. 5, 120). Cognition develops and advances through meaning in words and language. Meaning is essentially conceptual, and it is motivated by cognitive processes. The most fruitful approach to meaning is to regard it as conceptual in nature (Cruse, 1999). Thus the study of meaning in language and the function of the cognitive processes are "the only legitimate and scientific goal in the study of language" (Kövecses, 2005, p. 169).

In historical semantics and lexical semantics, the meaning development of a word, or the semantic change of a lexeme, has been supposed to be motivated by some mechanisms, such as metaphor, metonymy, synecdoche and irony, see e.g. Blank \& Koch (1999), Cuyckens et al (2003), Fisiak (1985), Nerlich (2010), Ullmann (1962). Metaphor and metonymy are generally recognized as the most vital mechanisms underlying meaning extension in cognitive semantics, see e.g. Cuyckens \& Zawada (2001), Nerlich et al (2003), Traugott \& Dashier (2002). Words are protean in nature in terms of meaning (Vyvyan Evans, 2009). A word gains more meanings through historical development, and those meanings are motivated and somehow related. When it is said that a word is polysemous, the meanings of a word are viewed synchronically at the present date; i.e. looking at it from a height, the historical developments accumulate. Polysemy hence is actually the "synchronic reflection of 
diachronic-semantic change" (Geeraerts, 1997, p. 6), and is treated as common in various levels of language such as lexicon, morphology, and syntax. Polysemy in the cognitive view is thus considered as "a fundamental feature of human language" (Vyvyan Evans \& Green, 2006, p. 36). Although it views meanings synchronicdiachronically, the polysemy view has yet to explicate meaning initiation and meaning development, as well as meaning structure, in a reasonable way.

This chapter will first examine views of word meaning and meaning organization, then identify the relationship between word meaning, word class and central knowledge. After that it will present a developmental view of meaning prototype, ${ }^{43}$ and argue that word meaning is in nature prototypical but the meaning prototype itself develops in use with a shifting central knowledge.

\subsection{Meaning prototype and cognitive processes}

\subsubsection{Views of word meaning}

Regarding word meaning, there are some well-known views, for example, meaning selection, and meaning creation. The meaning selection view holds that a word has many well-established meanings; the meaning in language use is selected from the mental lexicon. See e.g. Aitchison (2003), Evans (2009), Gorfein (2001), Nerlich \& Clarke (2003), Taylor (2006). Even if mental lexicon is also regarded as dynamic in terms of link between new and existing words and knowledge expansion (Aitchison, 2003), there are still some problems with the term "selection" and with this selection view, because it theoretically ignores dynamic meaning development, creativity of language use, and conceptual inference by emphasizing the finite number of established senses, ${ }^{44}$ not to mention that it does not pay much attention to how these senses in the lexicon are derived and organized. For instance, the meaning selection approach cannot offer an appropriate meaning from the established lexicon for "the Blues" in "The Blues were a total failure last weekend". "The Blues" here is used to mean the football team Chelsea, whose home kit colour is blue, and this meaning

\footnotetext{
${ }^{43}$ In this research I propose the term meaning prototype and prefer it to prototypical meaning, because prototypical meaning as used in the literature seems to indicate a fixed prototype of a meaning, as shown in the following section. Meaning prototype, just like prototype as argued here, is dynamic and developmental. In the development of word meaning, different meaning prototypes may turn up.

${ }^{44}$ Although some scholars hold that sense is different from meaning, in this research I do not make such distinction for the sake of analysis.
} 
cannot be found and selected right away in mental lexicon, because it is not one of the established meanings of "blue".

The meaning creation view asserts that meaning is created in the context without meaning selection, or frequently, mixed with meaning selection (Clark \& Gerrig, 1983). For instance, Clark \& Gerrig's example: "Please do a Napoleon for the camera". In order to understand "do a Napoleon" as "posing with one hand tucked inside their jacket à la Napoleon", one must first refer to Napoleon Bonaparte and search information about him for the specific act fitting the request in this context and then create a meaning around it; this interpretation is built entirely around elements from the knowledge of Napoleon's life, which are not entries in one's mental lexicon (Clark \& Gerrig, 1983, p. 592). According to Clark \& Gerrig (1983, pp. 591-592), eponymous expressions such as the above come in many forms (e.g. verbs, adjectives, common nouns) and you cannot select a meaning straightaway from the well-established lexicon, you must create a proper meaning for this kind of expression on the spot. But they also claim that in other contextual expressions, say, "You'll have to ask a zero", meaning creation is mixed with meaning selection: When the caller heard "zero", she was intended both to select the conventional meaning "naught" and to create the novel meaning "person you can reach on a telephone by dialling [naught]". This creation is actually based on the established senses, because "creating the intended sense cannot be truly complete until the right conventional sense has been selected (Clark, 1992, p. 364)". This view consists of two types of situation. In the first situation, meaning is completely created in the context, involving knowledge of the item which cannot be directly obtained in the mental lexicon. In the second situation, meaning creation in the context is based on meaning selection from the mental lexicon; it is actually a meaning development in terms of integration of the meaning selected from the lexicon and the contextual elements. It presupposes the existence of many meanings of a word in the lexicon for selection against the context where the word is used. This meaning creation view therefore makes a distinction between encyclopaedic knowledge and lexical meaning (linguistic knowledge), and meaning creation is a search for particular encyclopaedic knowledge and/or a selection of meaning in the lexicon and then an integration of these two with the contextual elements. 
The meaning creation view then transits to meaning construction view. This view holds that meaning is constructed in the mind of the language user, and linguistic units are just prompts used for constructing meaningful conceptual representations (Radden, et al, 2007a). Meaning construction is considered as "an online mental activity whereby speech participants create meanings in every communicative act on the basis of underspecified linguistic units" (Radden, et al, 2007a, p. 3). In this view, meaning is supposed to be constructed online because the linguistic unit is implicit and undetermined by virtue of the fact that it is not conventionally associated with particular meaning, or its meaning is vague, or it is not compatible with other units in meaning within the expression; thus in order to understand an expression, meaning construction is needed (Radden, et al, 2007a, pp. 5-9). This shows that the meaning construction view is a radical meaning creation view. The above creation view holds meaning creation takes place only after meaning selection does not succeed; this meaning construction view instead holds that meaning is constructed online without any attempt at meaning selection. It even deemphasizes the already existing meanings by stating only linguistic units rather than meanings the units already have. Although this approach claims not to restrict the meaning construction to novel meaning creation but cover any meaning representation, it actually does not tell how to interpret non-novel meaning representations (see Radden et al., 2007a, p. 4). Like Blakemore (1992, pp. 3-4), the meaning construction view treats linguistic units merely as clues to the meaning to be constructed in the context. It considers linguistic units as underspecified only to be fully specified in the context. But it neither tells why the linguistic units can become the clues, nor shows how they play their prompt role, nor elucidates how to deal with the novel meaning constructed online on the spot. Should the constructed meaning be piled together with the old meanings (if there are any in this view) as part of information in the linguistic prompt or just a temporary meaning used as once-for-all? Since meaning is constructed online, every meaning is novel, but what is the relation between the meanings constructed so far? If they are treated as old meanings, then what is exactly the relation between the online constructed meaning and the existing meanings? In a word, this view does not explicate word meanings in their formation, development and organization; rather it is more an interpretation of meaning understanding in context. 
While the idea that words have meaning(s) is present in both the meaning selection and the meaning creation views, the idea seems to fade away with the meaning construction view. Even further, some others, e.g. Allwood (2003), Croft \& Cruse $(2004)^{45}$, Evans (2006), overtly claim that words do not really have meanings, they have only "meaning potential" (Allwood, 2003) or "semantic potential" (Vyvyan Evans, 2006). The meaning potential is "all the information that the word has been used to convey either by a single individual or, on the social level, by the language community" and is "the union of individually or collectively remembered uses" (Allwood, 2003, p. 43). The meaning potential therefore contains both linguistic information and encyclopaedic information. Meaning potentials are reified to contextually determined meanings in activation through various cognitive operations in a context which creates certain conditions for the activation. Thus "the meanings which are actually constructed are always the products of memory activation and the application of contextually sensitive cognitive and/or linguistic operations on meaning potentials" (Allwood, 2003, p. 56). So in Allwood's view, the meaning potential is the collection of stored information a word carries in use, and meaning emerges from the activation of this information by the contextual elements. Whereas in employing "semantic potential", Evans first thinks "words do not have meanings in and of themselves" (Vyvyan Evans, 2006, p. 492); second, unlike Allwood, Evans distinguishes purely linguistic knowledge (information) from encyclopaedic knowledge (information). For Evans, the semantic potential is just the encyclopaedic knowledge that words provide access to. Both the outlooks of "meaning potential" and "semantic potential" stress the encyclopaedic knowledge (information). While Allwood treats it as information deriving from use of language and from other experience with the world, Evans treats it as distinct from stored linguistic knowledge, which he takes as linguistic prompts to semantic potential. ${ }^{46}$

\footnotetext{
${ }^{45}$ Croft \& Cruse (2004, pp. 97-98) put forward this word-no-meaning view, but their view is actually in line with meaning construction view proposed by Radden et al (2007). Croft \& Cruse take linguistic expressions as just one vital component (partial clues) of those required for the online meaning construal, which is conducted in integrating linguistic elements, non-linguistic knowledge, contextual information ("information available from the context, knowledge and conjectures regarding the state of mind of hearers and so on").

${ }^{46}$ This idea is very obvious in Evans' Theory of Lexical Concepts and Cognitive Models and in his exploration of meaning of words such as Evans (2006; 2007; 2009). However, elsewhere, e.g. Evans et al (2007), Evans and Green (2006), the distinction between linguistic knowledge and encyclopaedic knowledge is regarded not to exist and even linguistic knowledge is subsumed in encyclopaedic knowledge.
} 
Despite the different treatment of these two kinds of information, they both hold an activation view of the larger knowledge profile and a potential view of meaning. However, these views in the end also presuppose the existence of already stored uses or knowledge/information which they treat as the meaning/semantic potential for contextual activation and access. What is activated is the already existing (i.e. old) knowledge or information. These potential views hence, in the developmental sense, do not pay much attention to the dynamic course of both encyclopaedic knowledge and linguistic knowledge accumulation, though Evans (2006, 2007) intends to provide a theory of dynamic meaning construction and Evans \& Green (2006) see encyclopaedic knowledge as dynamic. Another point is that not all the stored knowledge of a word will be activated in an actual use, but the meaning potential view seems unaware of this problem. In addition, word meaning, in the view of Allwood and Evans, is actualized and determined in the context and co-text on the level of knowledge. Thus both Allwood and Evans seem to consider word meaning from the conceptual perspective. Yet they do not say how the situated utterance provides activation or access on the conceptual level or show how the potentials together contribute to meaning construal, since every element in the utterance has a meaning, or semantic, potential. Since knowledge (whether linguistic or encyclopaedic) plays a crucial role in meaning attainment, how is the knowledge (linguistic and/or encyclopaedic) organized or structured on the conceptual level? Just like encyclopaedic knowledge, linguistic knowledge originates from actual language use in a certain situation. Linguistic knowledge and encyclopaedic knowledge are therefore hard to delimit in the perspective of Cognitive Linguistics (both are conceptual and experienced at once) and thus the distinction between them cannot be maintained, but it is necessary to find a cognitive way to build up the knowledge as an organic whole entity such as prototype, category, and scenario. What is more, if a word has no meaning and only has meaning potential, where does this potential come from? In what way does it exist conceptually? Is there any kernel of the potential? Or how is the potential organized? If meaning is dispensable to a word, what is the value of the word in existence? It might be better to say that it is simply the meaning(s) a word has that lends the word potential to wide use and other contexts, motivated by some cognitive processes. Words do have meanings, which originate from the global/general conceptual knowledge the words bear in their 
initial conceptualization. It might be said that it is this global/general conceptual knowledge that bestows a word potential for a specific use.

\subsubsection{Meaning organization}

Thus mentioned, it follows that if a word generally has many meanings or a word has a meaning potential or semantic potential, then before the meaning of a word is determined or constructed in a context a question arises: How are the many meanings of a word or the semantic/meaning potential organized or structured?

It seems that the meaning creation view, the meaning and semantic potential views, and the meaning construction view have not provided any satisfactory answer to the above question. The traditional view of word meaning proposes that every word has a basic/core meaning (often regarded as the literal or the assumed primary meaning of the word), which is shared by all other meanings that are derived or generated through mechanisms of meaning extension as mentioned at the outset of this chapter. This meaning accommodation view sees the basic meaning as context independent and taking effect everywhere the word is used. But how to determine which meaning is the assumed basic or which is the assumed primary or literal? This is a rather tricky problem to tackle, especially in the argument of literal vs figurative meanings (Glucksberg, 2001; Katz et al, 1998; Ortony, 1993). The idea is even proposed that there is no literal meaning, since language is figurative in nature (Gentner \& GoldinMeadow, 2003; Gibbs, 1994; Lakoff \& Johnson, 1980). The traditional view is accordingly refuted by the meaning creation view, for details, see e.g. Clark \& Clark (1979), Clark (1992, pp. 303-380), and meaning/semantic potential views, see e.g. Evans (2007, 2009), Allwood (1981).

The abstractivist view holds that there is an abstract meaning for each polysemous word and that all other meanings of this word are derived from it through context, see e.g. Nerlich \& Clarke (2003), Gibbs (1994, ch.2), Cruse (1999, ch.7). This view, which assumes that there is a single very general and abstract concept of the word that can umbrella all other meanings in use, has also been rebutted, because words are "slippery customers" with "vague boundaries and fuzzy edges" (Aitchison, 2003, p. 52). For instance, Gibbs (1994) points out that in the following sentences 
The boy climbed the tree.

The locomotive climbed the mountainside.

The plane climbed to 30,000 feet.

The temperature climbed into the $90 \mathrm{~s}$.

The boy climbed down the tree.

We climbed along the wall.

"Climb" can hardly be said to have any common abstract meaning or semantic feature. For details, see Gibbs (1994, pp. 43-48). Another example in this point is "fast" in the following sentences (for details see Vyvyan Evans, 2009; Pustejovsky, 1998):

A fast car

A fast game

A fast book

A fast typist

A fast decision

A fast driver

The fast lane (of the motorway)

We need a fast garage for our car, as we leave the day after tomorrow.

Since a common abstract meaning cannot be reached, "prototype" is borrowed from psychology in word meaning analysis. Opposite to the above two meaning organization views, the prototype view, based on family resemblance, argues that there is no feature/concept necessarily shared by all the meanings of a word; the meanings are accommodated around the prototype (the best example or representative) in terms of degrees of similarity to it, e.g. Tsohatzidis (1990b), Nerlich \& Clarke (2003). In Nerlich \& Clarke (2003), almost every word is considered polysemous, and the meanings of a word are viewed as related to a prototype through metaphoric and metonymic associations with flexibility. But this prototype view just takes degree of similarity into account and like the above two views, establishes a core, i.e. the prototype as the reference point of comparison in telling which meaning is more representative and which is less so. It indicates this prototype is well established and totally static in any situation. This is problematic in reality. Family resemblance means that there is something inherited in this 
generation and maybe lost in the next generation and something new will be gained and inherited in a new generation, and so on and so forth. It stresses inheritance continuation and development continuum. So a static prototype meaning view is destined not to provide a satisfactory theory for a dynamic meaning development and organization process.

Within linguistics, the prototype approach is first of all applied to word meaning study (Tsohatzidis, 1990a). Prototype theory and its application in Cognitive Semantics are fruitfully explicated in Geeraerts $(1988,1989,1990,1997)$ and Taylor (1995, 2003). In lexical semantics prototype is generally assumed to be static; in cognitive semantics, however, the dynamic of prototype is actually mentioned. For example, in talking about prototypicality as an efficiency principle, Geeraerts (1997, p. 114) points out that it is "the dynamic nature of the synchronous notion of a prototypical conceptual organization" (emphasis original) that most likely provides "the most profound reason for the adequacy of prototype theory for specifying the characteristics of semantic change". But his dynamic nature of prototype is based on "the centralizing action of a conceptual kernel" upon a number of nuances. Geeraerts (1997, p. 114) says, "The multiple actualizability of a prototypical concept into variously deviant nuances marks it as an inherently flexible, dynamic structure." Therefore, in Geeraerts's view, the reason why prototype is dynamic is that the peripheral cases are not static but extensive and all these deviant cases are centred around a single core, i.e. the prototype; it is the expanding of these peripheral cases that determines the dynamic of prototype. It will be instead argued here that prototype is dynamic because the prototype itself is developing and changing, i.e. the kernel per se is not fixed but changing in the development.

In his analysis of "vergrijpen", Geeraerts (1997, p. 61) also finds that this word "contains two separate prototypes", "of which the most important one is itself made up of three smaller prototypical centres," and that "saying that the diachronic structure of "vergrijpen" is prototypical does not imply that there is one and only one prototypical concept that holds together the different chronological developments". But unfortunately Geeraerts (1997, p. 62) still maintains that "all the readings [i.e. senses] mentioned in the separate clusters can be considered variations of a single central meaning". He emphasises that, while the analysis of "legging" shows a 
category with a single prototypical centre, and the analysis of "vergrijpen" shows that a prototypical cluster may itself contain several salient sub-concepts, these additional subprototypical centres can be analysed in the similar way to the prototypical analysis of "legging". So Geeraerts's dynamic of prototype is basically that the prototype has a fixed centre and its dynamic relies on the indeterminate peripheral cases or members of the category which are mostly expanding. But since it is not likely to incorporate all meanings of a word under one prototype (Aitchison, 2003), that meaning prototype is not fixed. As will be argued later, the peripheral cases expand because the centre of the prototype is changing and developing, and a new centre/prototype will have a different range of categories. In the development of word meaning, new centres will show up and determine new prototypes, which will stand out as the figure, whereas the previous prototype centre will stand back in the ground, although it may be evoked and brought to the centre again in a certain context.

The above analysis shows that there are some commonalities shared by these meaning organization views. The first one is that these views in fact separate form from meaning in a word. This decomposition has done much harm to semantic analysis (Vygotsky, 1986, p. 4). A word is conceptualized in a situation not in terms of a simple form but in terms of a unity of form/sound and meaning. That is, a word is basically conceptualized as a unit of word-meaning. A word can never be fruitfully divorced from its meaning(s); even made-up and nonsense words bear some meaning in the context of use. Therefore the distinction between linguistic knowledge and encyclopaedic knowledge and the separation of form/sound from meaning are proved to be an artificial intervention. The second commonality is that all these views hold a polysemous view of word meaning but their interpretation of meaning organization for polysemous words is far from satisfactory. The existing prototype view, which seems to prevail in current meaning analysis, assumes the existence of many meanings and the existence of a prototypical meaning (a prototype in sense), which is supposed to be a fixed centre and thus context-independent, nondevelopmental, and abstract ideal representation of the meanings of the word. This view sees word meaning in a synchronic way and takes them all together as a heap and then analyses how these meanings are built together. But questions arise with this view. For example, is the prototypical meaning the assumed first/primary one or 
the abstracted essential one? If so, then what is the difference between this view and the abstractivist and traditional views? Thus the problem with the prototype view is how to see the prototype, and how to distinguish it from the assumed primary meaning as advocated in the traditional view or from the assumed abstract sense as claimed in the abstractivist view, if there is any relation. Besides, although the prototype view of word meaning might explain the organization of the meanings of a word to some extent, it does not explain word meaning formation and development, i.e. how the word meanings are derived and develop, how the prototypical meaning comes into being. Is it derived in the very beginning or in the development of word meaning? What is most important is how to empirically identify the prototypical meaning. ${ }^{47}$

In contrast to the centre-fixed prototype view and the non-meaning (potential) view, this research, concurring with Ungerer \& Schmid (2006), holds a developmental prototype view of word meaning. Ungerer \& Schmid illustrate prototype shift and prototype split by analysing the categories $\mathrm{COACH}$ and IDEA respectively in diachronic contexts. In prototype shift, the central attributes of a category are replaced usually due to extra-linguistic changes. The category prototype of $\mathrm{COACH}$ in the $17^{\text {th }}$ century is STATE CARRIAGE, which is shifted to STAGE COACH in the $19^{\text {th }}$ century and to MOTOR COACH in the $20^{\text {th }}$ century. Prototype split involves obtaining specific prototypes from a global prototype, which is shown in IDEA from CONCEPT to CONCEPT and BELIEF; to CONCEPT, BELIEF, AIM and INSPIRATION. See Ungerer \& Schmid (2006, pp. 316-319) for details. In their view, the social and cultural context exerts crucial impact upon the whole internal structure of a category; extra-linguistic changes result in the shift of central attributes or replacement of a category. Their insight and analysis of prototype shift and prototype split is suggestive and far reaching. For instance, diachronic analysis and syntactic environment can be taken as ways of identifying meaning prototypes.

\footnotetext{
${ }^{47}$ Psychological experiments show that we think in the form of prototype; it seems a prototype is established in our thinking without conscious awareness. Word meaning analysis therefore needs to identify the prototype established and employed in the particular context in the first place. However, since prototype is dynamic and developmental depending on the functions of the word in use, prototype identification is a great challenge. It might be beneficial to adopt a diachronic approach together with a corpus-based collocation method, consisting in a combination of a study of corpus concordances with a systematic analysis of the relevant polysemy networks registered in authoritative dictionaries and, ideally, with psycholinguistic experimentation. But this goes way beyond metonymy research and will not be discussed here due to non-relevance and space limitations.
} 
Nevertheless, the shift and split of prototype is not the result totally of changes in extra-linguistic context. The internal structure of information or semantic values, or rather the conceptual knowledge, cannot be neglected. It is actually the impact from both the internal and the external with the internal more crucial in weight. It is just like conceiving a baby with a woman producing eggs as a precondition. It is a sort of “conceptual integration" (Fauconnier \& Turner, 1999). Furthermore, central attributes cannot shoulder the burden of representing the category on their own, they are just qualia structure, in Pustejovsky's (1998) term; there is still functional, eventual, relational information, or knowledge of entities, qualities and functions, in Ahren's $(2002,2010)$ terms. Central conceptual knowledge here includes all these aspects, as argued below. Moreover, it is argued that it is the knowledge that a word accumulates that probabilizes an active and developmental meaning prototype.

This chapter argues that the change of word meaning is because the prototype develops in word use. Word meaning originates from its conceptualization and emerges in the shape of a concept. Concepts are formed and represented in prototypes (Eleanor Rosch, 1975). Prototypes originate from experienced reality through the function of cognitive mechanisms such as metonymy and metaphor. See also Geeraerts $(1988,1989)$. With the accumulation and enrichment of experience, the internalized and generalized reflections of the experience of reality are developed and specified, and hence the meaning prototype is developed in the use of a word. Meaning prototype is thus dynamic and functional. A word may develop different meaning prototypes through metonymy and metaphor.

Since the appearance of prototype theory, it has been generally supposed (with the exception of Ungerer \& Schmid (2006) as analysed above) that there is only one prototype in a category (i.e. a category contains a member which best represents the category), which can be illustrated in the above analysis of the prototype view of word meaning. However, recent study shows that it is possible to have two or multiple prototypes in a category, for example, the Tsonga colour term "rihlaza" refers to a green-blue continuum, but appears to have two prototypes, a focal blue, and a focal green. ${ }^{48}$ Geeraets (1997) also mentions this point in the lexical semantic

\footnotetext{
${ }^{48}$ Concept formation. (2008, June 18). New World Encyclopedia. Retrieved 01:29, March 25, 2010 from http://www.newworldencyclopedia.org/entry/Concept_formation?oldid=736189.
} 
analysis of "vergrijpen" that there are several (sub)prototypical centres in its meanings as shown above. Prototype itself is functional, and it develops in conceptualization and in the use of a word, which is best represented in word meaning. When a new entity or word, even a very concrete entity, is encountered in a situation, e.g. a desk, a concept of it is formed in the situation by generalization and abstraction primarily through metonymy. But because it originates from only one particular, this (generalized) concept is rather primitive, and then it will become specific after the experience with this category increases, yet the general and global concept remains and becomes "first-in last-out" in memory, even in memory breakdown, see for example Mandler (1993, 1998b, 2004a, 2008) and Vygotsky (1986). In this sense, as argued in Chapter 3, concepts develop from global and abstract to specific, and in this conceptual development, prototypes surely develop as well. Both prototype split and prototype shift belong to this process. Meaning changes as a result of meaning prototype development and different combinations of categories, or rather, re-categorization. Categorization is an internal feature of human cognitive process. Human beings have an insatiable appetite for categorization (Trenholm \& Jensen, 2004, p. 144). The capacity to categorize is the most fundamental of human capacities (Lakoff, 1987b, p. 8). However, categorization is a dynamic process which keeps (re-) categorizing all the way; so does prototype.

This conceptualization process from general and global to specific and concrete seems contradictory to the widely held intuitive opinion that conceptualization is from concrete to abstract. Research results of cognitive psychology and developmental psychology evidence the global-to-specific conceptual formation and development since infancy and throughout the life. See Mandler (1993, 1998b, 2004a, 2008), as pointed out in Chapter 3. Grammaticalization, in which some content words become functional words, might immediately be thought to sit against this cognitive process. However, as argued in the previous chapter, content and function words are simply viewed from the perspective of grammatical function, not from conceptual perspective. In the conceptual view, the fact that content words become function words is self-evidence of dynamic meaning prototype. The peripheral knowledge in the primitive prototype is brought to the focus position in use for some purpose or goal, thus developing a new prototype with the peripheral becoming the central knowledge and with a new category extension. This dynamic 
meaning prototype is not determined once and for all, it does develop from generation to generation in terms of family resemblance.

The following section will demonstrate the relevance of the above discussion through a cognitive analysis of " 110 " in China aided by a corpus-based approach. Prototype per se is a functional and prototypical concept which arises and develops in use and the developmental prototype determines the scenario ranges a word can apply to, even with different syntactic representation, e.g. part of speech. This is because every event scenario is a usage-based event consisting of things and situations, and situations can be temporal units involving aspect, tense and modality, or relational units involving event schemas and spatial extensions (Radden \& Dirven, 2007). This prototype view of word meaning from the conceptual perspective is also because conceptually the meaning prototype of a word accommodates all the uses in different syntactic environments and different contexts through some underlying cognitive mechanisms such as metonymy and metaphor.

The rest of this chapter is arranged as follows. Section 6.3 discusses relations between different parts of speech and argues that meaning itself is a prototypical concept and all the meanings deriving from different parts of speech can be conceptually accommodated in the meaning prototype of the word. Section 6.4 introduces 110 as police emergency number in China and analyses its extended use in a corpus motivated by metonymy and metaphor. Bases on the analysis, it comes to the conclusion that meaning prototype as a concept itself is functional and developmental, in addition to prototypical, and it arises and develops in use and this dynamic conception of prototype empowers it to have both flexibility and stability enough to bear adequate explanation and description for meaning change.

\subsection{Meaning as a prototypical concept}

\subsubsection{Meaning, word class and central knowledge}

Traditional study of meanings of a word usually focuses on the word as a certain part of speech such as noun, adjective, or verb. If the meanings of a word can be associated with different parts of speech, the word will be determined as homonymous rather than polysemous, see e.g. Allwood (2003). In Conceptual 
Metaphor Theory, parts of speech of the words in the source domain are not taken into account. For example, the list of sentences under ARGUMENT IS WAR in Lakoff \& Johnson (1980, p. 4) includes adjective, verb (phrase), preposition (phrase), noun, etc. Perhaps partly because this theory is presented from the conceptual perspective of semantics, the relation between different parts of speech of a word is not pointed out. Deignan (2006), through a large corpus study, points out that metaphorical uses of words show differences in their grammatical behaviour, or even word class, e.g. it is quite common that the literal and metaphorical meanings of a word are of different word classes. She also suggests that individual word forms are metaphorically mapped. This means that word meaning can conceptually embrace different classes of the word, as will be illustrated below in the analysis of " 110 " in China, in which " 110 " is used in reality as noun, adjective and adverb. Conceptually these word classes can be subsumed into a meaning prototype through metonymy.

While Conceptual Metaphor Theory specifically studies metaphors with a target domain characterised by a number of source domains, for example, ARGUMENT IS JOURNEY /BUILDING /CONTAINER /WAR; HAPPINESS IS UP/LIGHT /VITALITY /OPPONENT /INSANITY, see e.g. Kövecses (2008) and Lakoff \& Johnson (1980), Kövecses (2000) proposes a study of metaphors in which a single source concept can characterize many distinct target domains, for instance, BUILDING can be used to characterise different target domains such as THEORIES, RELATIONSHIPS, CAREER, SYSTEMS, LIFE, GROUPS, i.e. THEORIES ARE BUILDINGS, RELATIONSHIPS ARE BUILDINGS, A CAREER IS A BUILDING, A COMPANY IS A BUILDING, ECONOMIC SYSTEMS ARE BUILDINGS, A LIFE IS A BUILDING, SOCIAL GROUPS ARE BUILDINGS.

This proposal is significant. If the reason why a single target concept is understood or interpreted via several source concepts is that the target has a number of distinct aspects to be addressed (Lakoff \& Johnson, 1980), then this reason also applies to the case in which a single source concept is used to characterize different targets, because every concept, whether it acts as the source or the target, is a multidimensional entity with different aspects when viewed from different perspectives. The proposal thus presents the reverse of the Conceptual Metaphor Theory and gives a successful explanation of these two phenomena at the conceptual 
level. The question here is what guides this source-to-target mapping or what makes an entity or event a source characterizing different targets or vice versa. Whether it is mapping from one source to many targets or from many sources to one target, there must be underlying reasons for the domain mappings ${ }^{49}$, i.e. mapping principles for conceptual metaphors to take place (Ahrens, 2002, 2010; Gong, Ahrens, \& Huang, 2008). Different mapping pairings, especially many sources for a single target, select and thus highlight different aspect of the domain knowledge, which is generally the presupposition of the mapping pairing. For instance:

LOVE IS FIRE: Love is understood as fire because fire involves burning with physical light and warmth and love involves giving emotional light and warmth.

ANGER IS FIRE: Anger is understood as fire because fire involves physical burns and anger involves emotional burns. See Ahrens (2002, pp. 289-290).

IDEA IS BUILDING: Idea is understood as building because buildings involve a (physical) structure and ideas involve an (abstract) structure.

IDEA IS FOOD: Idea is understood as food because food involves being eaten and digested (by the body) and ideas involve being taken in and processed (by the mind). See Ahrens (2002, pp. 279-282).

But exactly what makes the mapping happen between domains? Kövecses (2000) suggests it is a particular meaning focus (or foci) that plays the role, and this meaning focus (or foci) is (are) constituted by the central knowledge of the source shared by a speech community, and it is inherited by the target as well. Following Langacker (1987, pp. 158-161), Kövecses considers central knowledge as knowledge (about an entity or event) that is conventional, generic, intrinsic, and characteristic. Of these four criteria for central knowledge, "conventional" considers whether the knowledge is general knowledge in the community; "generic" considers whether the knowledge applies to all entities in the category; "intrinsic" means the knowledge applies to the entity itself only; "characteristic" means the knowledge only applies to the entities in the category. In this defining notion of central knowledge, these four

\footnotetext{
${ }^{49}$ Although the problems of "domain" and "mapping" are pointed out in Chapter 2, here the analysis is on CMT, these terms are used to show some points held in the cognitive domain approach.
} 
criteria are used to measure the centrality of knowledge. But it is inevitably hard to manipulate owing to the abstractness, generalness, and fuzziness of the criteria. For example, see Croft (2002), Ruiz de Mendoza (2000) and Ruiz de Mendoza \& Campo (2002). Ahrens $(2002,2010)$ advocates three aspects of real world knowledge of the domains in analyzing domain mapping pairings, i.e. entities (what entities do they have), qualities (what qualities do they or the entities in the domain have), and function (what do they do or what can they do to or in the domains). These three aspects are more viable for experimental manipulation, i.e. linguistic analysis, because they are not only related to real world knowledge but also closely related to language use, i.e. the established usage patterns and their associated practices (Taylor, 2006), and because both conceptual knowledge and lexico-grammatical constructions (e.g. lexical collocation) account for the use (Gibbs \& Matlock, 2001). Due to the experimental manipulation of the three aspects and the corresponding relationships between nouns/subjects/objects and entities, adjectives/adverbs/modifiers and qualities, and verbs/predicates and functions, "entity", "quality" and "function" will be employed here as aspects in deciding meaning prototype, but add a third point for "function", i.e. function indicates what they do or what they can do to or in the domain or what can be done to them in the domain.

It is suggested that it is this central knowledge regarding entity, quality and function that probabilizes the metaphorical and metonymic meaning extensions of a word and between different parts of speech of the word. But this central knowledge is not invariable. Kövecses's point is presented from the synchronic perspective, which ignores the diachronic development of the central knowledge, just like the abstractivist view and the static prototype view. It would be proposed that this central knowledge, which establishes the meaning prototype, originates from the conceptualization of the word in use, but it develops in more situated uses while staying comparatively stable, just like family resemblance and family inheritance mentioned above. In the development process different aspects of the central knowledge will pop out as dominant through metonymy in actual use and thus establish a new meaning prototype with this dominant part as the central knowledge of it. That is the reason why some words lose the original prototypical schemata and 
even take some new meaning prototypes, as evidenced and supported by historical semantics and lexical semantics, for example:

(41) My dear sister was talking about you only today at tea. (British National Corpus, hereafter BNC)

(42) Grow your own tea with the Tea Plant from The Stroke Association. (BNC)

(43) I left them eating their tea. (BNC)

(44) I'd like to place an order for some tea with you.

(45) Would you like a cup of tea?

(46) Tea was a large meal for the Wicksteeds. (Dirven, 2002)

As pointed out by Dirven (2002) and illustrated in the examples, "tea" develops from originally denoting a plant to the product (the leaves), from the leaves to a drink, further to the occasion when the drink is consumed, finally to meal.

\subsubsection{Meaning prototype}

Meaning as a prototypical concept can accommodate different parts of speech of a word in use. Studies in historical semantics, cognitive semantics, and corpus linguistics have demonstrated that the meaning of a word when used in a different word class is conceptually related and connected by cognitive mechanisms such as metonymy and metaphor. In actual language use, there are a lot of denominal verbs, i.e. there are lots of situations where "nouns surface as verbs" (Clark \& Clark, 1979), and vice versa. For example, he needs to "bing" or "baidu" (the largest Chinese search engine) some information on the internet; he "headed" the ball into the goal. Language users are very creative and innovative consciously and unconsciously in using words and assign their functions for maximum effect in real utterances, depending on the perspective the users take in their conceptualization of the situation (Janssen, 2003). For instance, the English genitive, possessive pronouns and the preposition "of" can be used to express inexhaustible relationship, e.g. factual usership, element-set, mental property (Janssen, 2003). Thus the meaning of a word can be conceptually taken through some cognitive mechanisms to express a process 
or an action, an entity or event, and a relation, i.e. entities, qualities and functions, which constitute a certain cognitive scenario ${ }^{50}$.

As mentioned earlier in this chapter, words are in nature conceptual generalizations of the experience of reality; they are concepts, which change or develop together with human experience. Therefore, meaning is constructed at the conceptual level and "meaning construction is conceptualization" (Evans, et al. 2007a, p. 8). Conceptualization takes place in actual event scenarios. Different event scenarios may result in different conceptualization of the same word. Some scenarios happen to everyone in a community, some may just occur individually; thus some concepts are common to a speech community, and some concepts are particular to an individual. By the same token, the similar scenarios may bring about different conceptualizations in individual minds. Concepts take the shape of prototypes (Rosch, 1975), prototype per se develops, and concepts are themselves developing, meaning is accordingly dynamic and changing. Words exist in terms of meaning, i.e. concepts, meaning prototype is hence not static and established once and for all. But for most words, it is very difficult to track the development of the meanings. In the next section, "110" in China will be taken as a case study to see the development of meaning prototype so as to have a better understanding of meaning prototype and its dynamic nature. The reasons for choosing " 110 " are:

1) The meaning development of " 110 " is retrievable within the span of living memory;

2) It is used pervasively across the board in current China and has become a particular social feature;

3) As a symbol, "110" came into use just like other linguistic symbols with arbitrariness (if language is defined as an arbitrary system for human communication), or with motivation (in the sense that language did not come to existence for no reason);

\footnotetext{
${ }^{50}$ Although some scholars consider scenario as the same as frame (Fillmore, 2006(1972)), Idealized Cognition Model (ICM) (Lakoff, 1987b), e.g. Panther \& Thornburg (1998), throughout this thesis, scenario is viewed as event scenario, a basic unit in which cognitive activities occur, consisting of subject, object, action, motion, time, space, etc, and the components are related to each other by metonymy and are also metonymically related to the cognitive activity as a whole, also see Panther \& Thornburg (1998, p.756).
} 
4) Dynamic study of its extensive use in Chinese society can uncover the underlying conceptual mechanism(s) that motivate(s) its persvasiveness.

\subsection{The case of " 110 " in China}

\subsection{1 "110" as an emergency telephone number in China}

Emergency telephone numbers are adopted all over the world; they are typically a three-digit number, such as $111,999,911$, and 112 . Some countries employ only one national emergency number for police, fire and medical, such as 111 in New Zealand, 911 in United States, 000 in Australia, and 112 in most member countries of the European Union. Some countries employ different national emergency numbers for police, fire and medical respectively, such as Egypt (122, 180, and 123), Vietnam (113, 114, and 115), Brazil (190, 193, and 192). In China 110, 119, and 120 are employed as the emergency numbers for police, fire and medical respectively. "110" as a police emergency number is also used in Taiwan, Indonesia, Iran, Japan, Guatemala, and Bolivia. ${ }^{51}$

In China, "110" was first adopted as an enemy and spy report number in 1973 in place of the previous confusing " 00 " and then " 01 ". The reason was that it provided the shortest dialling time in that period. ${ }^{52}$ In 1982 it was used as the criminal report number in Guangzhou City. ${ }^{53}$ In the following few years, " 110 " was only used for criminal cases, e.g. murder, burglary. With social development, in 1986 the first "110" police service centre was established in Guangzhou, then gradually " 110 " was used as the emergency number for police assistance and service consisting of criminal, public security cases and other emergency situations as well. The "110" police service centre handles criminal reports, emergency assistance, and public reports and complaints against policemen and officials. With its function expanded from mere criminal cases to other emergency situations, "110" has now become the established emergency phone number for police assistance in China and is likely to

\footnotetext{
${ }^{51}$ See http://en.wikipedia.org/wiki/Emergency_telephone_number, retrieved on 11 Nov, 2009

${ }^{52}$ See http://www.thebeijingnews.com/news/reform30/2009/01-10/042@100949.htm, retrieved on 10 Dec, 2009

${ }_{53}$ Please note here, there are some changes in the development of the Public Security department since the founding of the People's Republic of China in terms of function (reduction and/or expansion in function). The years mentioned in the chapter reflected the changes as well, so do the categories.
} 
become the umbrella number for police, fire and medical emergency in the future, according to the reports.

\subsection{2 " $110 "$ " in corpus: Metonymical extension}

The above is in brief the historical development of "110" in China. In order to know exactly how "110" is currently used in China, a search of corpus Internet-ZH in Sketch Engine was conducted. ${ }^{54}$ The concordance of " 110 " as the query is 4674 , but the hits for " $110 "$ as a numeral involving the emergency service total only 1344. Then those hits were sorted out according to the syntactic value of " 110 " as used in the corpus. The findings show that " 110 " functions syntactically as noun, adjective, and adverb, with overwhelming quantity of 1017 hits as noun, 324 as adjective and 3 as adverb.

The analysis also shows that in the syntactic value of noun, "110" represents different semantic values, i.e. meanings. It functions semantically to represent different entities, as shown in Figure 1 below, such as the emergency phone number per se, the service centre, the service working system, the police station, the individual policemen, the police team, the police vehicle, the notice/call given by the service centre, and events involving "110" service. Below are some examples with original Chinese first, followed by Chinese Pinyin transcription and English translation:

(47) 自行车不见了。张先生于是拨打了 110 报警电话。(Noun, emergency police phone number)

Zi4 xing2 che1 bu2 jian4 le3. zhang1 xian1 sheng1 yu2 shi4 bo2 da3 le3 110 bao4 jing3 dian4 hua4.

With the bicycle gone, Mr. Zhang dialled "110" to report to the Police.

\footnotetext{
${ }^{54}$ See http://ca.sketchengine.co.uk/auth/preloaded_corpus/i-zh/ske/first_form, retrieved on 15 Nov, 2009. Internet-ZH is a Chinese web corpus with a size of 90 million words collected by Serge Sharoff, who thinks that internet-based corpora from search engines are better than balanced/ representative corpus such as BNC in terms of authorship, mode, audience, aim, and domain. Such internet corpora are generally established in four steps: word selection, query generation, downloading and postprocessing. See Sharoff $(2006,2007)$. Internet-ZH, as one of his internet-based corpora, was established in this way in 2005. It was tokenised and part-of-speech tagged using tools from North Eastern University, China. The following examples are taken from this corpus with kind permission. It is also available on his site at Leeds University, UK.
} 
(48) “你们不要乱来, 我要报 110 了。”(Noun, “110” service centre)

"ni3 men2 bu2 yao4 luan4 lai2, wo3 yao4 bao4 110 le3"

"Don't act foolishly, or I will report to '110'."

(49) 临下班时接到的 110, 一处住宅区有人跳楼自杀。(Noun, notice/call from " 110 " service centre)

Lin2 xia4 ban1 shi2 jie1 dao4 de1 110, yi1 chu4 zhu4 zhai2 qu1 you3 ren2 tiao4 lou2 zi4 sha1

Around finishing time, I got a call from "110", saying someone leaped to death in a residential area.

(50) 大家不要怕, 我已经通知 120 了, 110 也马上就到。(Noun, individual policemen)

Da4 jia1 bu2 yao4 pa4, wo3 yi3 jing1 tong1 zhi1 120 le3, 110 ye3 ma3 shang4 jiu4 dao4.

Don't panic, I have already reported to 120 , and " 110 " (i.e. policemen) will be here very soon.

(51) 大小车辆还是络绎不绝, 警方 110 已经管制了急诊门口。(Noun, police team)

Da4 xiao3 che1 liang3 hai2 shi4 luo4 yi4 bu2 jue2, jing3 fang1 110 yi3 jing1 guan3 zhi4 le3 ji2 zhen2 men2 kou3.

With a lot of traffic passing by in an endless stream, "110" (the "110" police) had controlled the doorway to the Emergency Treatments.

(52)（他）只顾自己讲电话, 连 110 的事没敢再提。 (Noun, event involving "110" service)

zhi3 gu4 zi4 ji3 jiang3 dian4 hua4, lian2 110 de2 shi4 mei2 gan3 zai4 ti2 
He kept talking on the phone and did not mention the "110" (event) any more.

(53) 其中一个小偷 抓住了, 并送往了 110。 (Noun, police station)

Qi2 zhong1 yi1 ge4 xiao3 tou2 zhua1 zhu4 le3, bing4 song4 wang3 le3 110.

One of the thieves was caught and sent to "110".

(54) 我们有监视和报警系统, 跟 110 联网, 派出所还派 2 名治安员 来...... (Noun, “110” service working system)

Wo3 men2 you3 jian1 shi4 he2 bao4 jing3 xi4 tong3, gen1 110 lian2 wang3, pai4 chu2 suo3 hai2 pai4 2 ming2 zhi4 an1 yuan2 lai2

We already have monitoring and alarm systems, which are connected with "110", and the local police station will send us 2 security personnel.

(55) 男子与售票员大打出手，并惊动了 110 。警察很快呼啸而来。 (Noun, police)

Nan2 zi3 yu3 shou4 piao4 yuan2 da4 da3 chu2 shou3, bing4 jing1 dong4 le3 110. jing3 cha2 hen3 kuai4 hu1 xiao4 er2 lai2.

The man started fighting with the conductor, which alerted "110" (the police). A police car screamed in at once.

(56) 一辆 110 开了过来，把我们抓住了。(Noun, “110” vehicle)

Yi4 liang3 110 kai1 le guo4 lai2, ba3 wo3 men2 zhua1 zhu4 le.

A "110" (car) came and we were caught.

Figure 6.1 below shows the semantic value of " 110 " as used as a noun in the corpus. 
Figure 6.1: The semantic representation chart of "110" as a noun

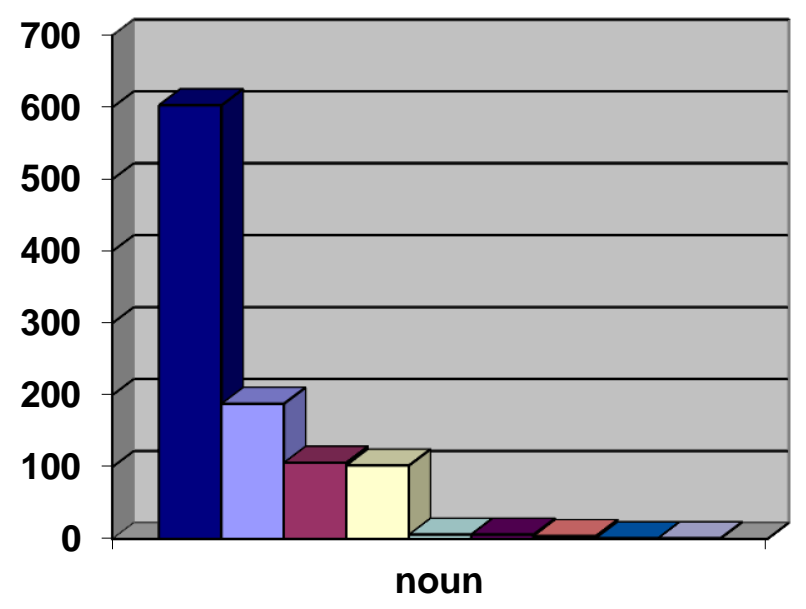

\begin{tabular}{l}
\hline uemergency number \\
$\square$ service centre \\
$\square$ police team \\
$\square$ police individual \\
$\square$ service system \\
$\square$ police station \\
$\square$ police vehicle \\
$\square$ events \\
$\square$ notice/report/call
\end{tabular}

This figure shows that when " 110 " is used as a noun, emergency phone number is represented the most in real discourse, the second is service centre, followed by police team, police individuals, service system, police station, police vehicle, event and notice/call given by the centre.

But the semantic value of "110" as adjective, i.e. quality, is less than as noun. Such semantic functions involved are as service system, as service centre, as police team, as individual policeman, as "110" report, and as spot (case site). For example:

(57) 开车的女士随后被赶来的 110 警察带走...... (Adjective, police team) Kail che1 de nv3 shi4 sui2 hou4 bei4 gan3 lai2 de 110 jing3 cha2 dai4 zou3...

The lady driver was taken away by the "110" policemen who came soon after the accident...

(58) 值班人员必须接收，市局“110”平台上的电脑才会显示对方...... (Adjective, service system)

Zhi2 ban1 ren2 yuan2 bi4 xu1 jie1 shou1, shi4 ju2 110 ping2 tai2 shang4 de dian4 nao3 cai2 hui4 xian3 shi4 dui4 fang1

The operator on duty must accept to have the caller's number shown in the computer of the bureau's 110 system platform... 
（59）民警小王曾经创造了一天出十八次 110 现场的纪录。 (Adjective, "110" case spot)

Min2 jing3 xiao3 wang2 ceng2 jing1 chuang4 zao4 le yi4 tian1 chu1 shi2 ba1 ci4 110 xian4 chang 3 de ji4 lu4

Policeman Xiao Wang has set a record of showing up on 18 "110" scenes a day.

（60）实验分析案情时, 再度接到分局 110 指令: 在长虹公园角落的草地 上...... (Adjective, “110" service centre)

Shi2 yan4 fen1 xi1 an4 qing2 shi2, zai4 du4 jie1 dao4 fen1 ju2 110 zhi3 ling4: Zai4 chang2 hong2 gong1 yuan2 jiao3 luo4 de cao3 di4 shang4

When analysing the case, they received another 110 order from the branch bureau: On the corner grassland of Changhong park...

（61）在被抬上救护车的时候, 110 同志和蔼的询问我为什么会被打 成...... (Adjective, individual policeman)

Zai4 bei4 tai2 shang4 jiu4 hu4 che1 de shi2 hou4, 110 tong2 zhi4 he2 ai3 de xun2 wen 4 wo3 wei4 shen 2 me hui4 bei4 da3 cheng2

While I was lifted into the ambulance, the 110 policeman asked me kindly why I was beaten to this...

(62) ......挤压犯罪空间。通过加强对 110 警情高发地区的研究分析...... (Adjective, “110"report)

..... Ji3 ya1 fan4 zui4 kong1 jian1. tong1 guo4 jia1 qiang2 dui4 110 jing3 qing2 gao1 fa1 di4 qu1 de yan2 jiu4 fen1 xi1.....

... squeeze the crime space. Through intensive research analysis of the area where 110 reports occur frequently...

Figure 6.2 shows this point: 
Figure 6.2: The semantic representation chart of " 110 " as an adjective

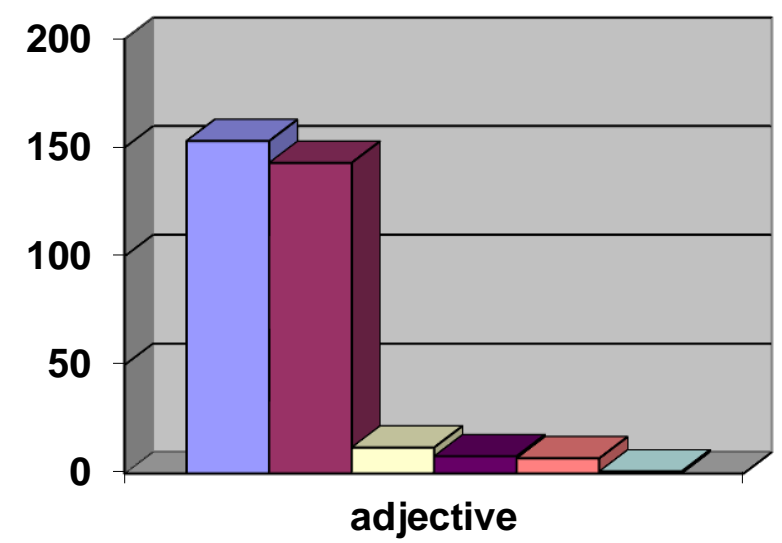

\begin{tabular}{|l|}
\hline$\square$ servie centre \\
$\square$ police team \\
$\square$ service system \\
$\square$ report \\
$\square$ individual policeman \\
$\square$ spot
\end{tabular}

In terms of semantic value, however, "110" as an adverb modifying "reporting" verbs (thus still in the aspect of quality) only involves the representation of the emergency phone number, indicating reporting to the police "by dialling '110". For example:

(63) 像这种问题是不是可以 110 报警当侵犯私人财产来处理呀?

Xiang4 zhe4 zhong3 wen4 ti2 shi4 bu2 shi4 ke3 yi3 110 bao4 jing3 dang1 qin1 fan4 si1 ren2 cai2 chan3 lai2 chu3 li3 ya4?

Can I report such incident to " 110 " in terms of personal property torts? ${ }^{55}$

(64) 于是找物业开证明, 110 报案，保险公司报案......

Yu1 shi4 zhao3 wu4 ye4 kail zheng4 ming2, 110 bao4 an4, bao3 xian3 gong1 si1 bao4 an 4 ...

Then I went to the Property Management Office for confirmation, reported to "110" and the insurance company...

(65) 于是我立刻 110 报案，…..

\footnotetext{
${ }^{55}$ Owing to the numeral nature of "110", I can hardly translate these examples into English in the same way (as an adverb) as it is used in the original utterance, thus I just adopt free translation here by either "reporting to 110 " or "reporting to the police by dialling 110 ", but the latter is too awkward as a translation though it gives the full meaning indicated.
} 
Yu2 shi4 wo3 li4 ke4 bao4 an4, .....

Then I reported to " 110 ” right away, ...

Within the noun category, " 110 " as a numeral, i.e. the emergency number per se, can still be separated from the rest. The number of " 110 " as the emergency police phone number is 603 , which overwhelmingly functions as the object of such verbs as "bo2 (make)" and "da3 (dial)". The number of "110" as a noun rather than a numeral is 414 ; " 110 " as an adjective/modifier is 324 ; and " 110 " as an adverb is only 3 . The following table provides a full breakdown:

Table 6.1: The numbers and percentage of 110 as different parts of speech

\begin{tabular}{|c|c|c|c|c|c|c|}
\hline \multicolumn{2}{|c|}{ Word class } & representation & \multicolumn{2}{|c|}{$\begin{array}{l}\text { Quantity } \\
\text { (1344) }\end{array}$} & \multicolumn{2}{|c|}{$\begin{array}{l}\text { Percentage } \\
(\%)\end{array}$} \\
\hline \multirow{9}{*}{ noun } & Numeral & Emergency phone number & 603 & 603 & 45.54 & \\
\hline & \multirow{8}{*}{ other } & Service centre & \multirow{8}{*}{414} & 188 & 45.41 & \multirow{8}{*}{31.27} \\
\hline & & Service system & & 6 & 1.45 & \\
\hline & & Police station & & 6 & 1.45 & \\
\hline & & Police team & & 106 & 25.60 & \\
\hline & & Police vehicle & & 4 & 0.97 & \\
\hline & & Police individual & & 102 & 24.64 & \\
\hline & & Events involving 110 service & & 1 & 0.24 & \\
\hline & & $\begin{array}{l}\text { Notice/report/call given by the } \\
\text { centre }\end{array}$ & & 1 & 0.24 & \\
\hline \multirow{6}{*}{\multicolumn{2}{|c|}{ adjective }} & Service centre & \multirow{6}{*}{324} & 153 & 47.22 & \multirow{6}{*}{24.47} \\
\hline & & Police team & & 143 & 44.14 & \\
\hline & & Service system & & 12 & 3.70 & \\
\hline & & Report & & 8 & 2.47 & \\
\hline & & Police individual & & 7 & 2.16 & \\
\hline & & Case spot & & 1 & 0.31 & \\
\hline \multicolumn{2}{|c|}{ adverb } & Numeral & 3 & 3 & \multicolumn{2}{|l|}{0.22} \\
\hline
\end{tabular}


This table shows that when the NUMBER category of "110" and the PHONE category and further the POLICE category combine, a new meaning prototype turns up, i.e. POLICE EMERGENCY. This meaning prototype consists of phone number, emergency service centre, emergency service police, emergency service system, emergency service vehicle, emergency service report, emergency service spot, emergency service report, and police station. All these meanings are accommodated around the emergency service through the emergency phone number " 110 " by metonymy. Thus it is very natural in real discourse that " 110 " functions, in terms of semantic value, as phone number, system, station, police (policeman), police vehicle, events, and notice/report/call, and syntactically as noun (numeral), adjective, and even adverb.

This synchronic situation of "110" used as different parts of speech in real discourse can be interpreted from the diachronic perspective. "110" as a numeral, i.e. the emergency phone number, is the most in quantity, for it was used initially as a phone number for emergency from the number category, whether motivated or not. " 110 " as a noun representing different entities is the second in quantity, for the emergency number was later used as a platform system and in an established service centre specifically for police. After the noun use, "110" is able to be used as an adjective, talking about the centre, the system, and the police, etc. This diachronic sequence accords with language use in terms of cognition development. The corpus did not show any "110" used as a verb. The reason might be that numerals are in nature easily used to stand for what they conventionally represent such as entity and quality, so the meaning prototype of such numerals is essentially nominal. But due to the collocation of "110" as a phone number with some verbs such as "make", "dial", it can finally be used as adverb in front of verbs indicating police reporting in real discourse. The analysis shows that the extension of syntactic value of " 110 " is centred on its use as noun, i.e. both its adjective use and its adverb use are from its noun use.

From noun to adjective and from noun to adverb, "110" is motivated by a metonymic cognitive process. That is, metonymy underlies "110"'s syntactic extensions both from noun to adjective and from noun to adverb, and motivates "110"'s semantic extensions from phone number to service centre, to police station, 
to police (policeman), to service police vehicle, to the service case spot and report/call/notice. The meaning development is related through metonymy as obviously shown in the above diagram, i.e. "110" as the police emergency number is metonymically related to represent police related entities (and their quality) such as team, person, system, station, report, vehicle, the case spot, etc. This finding is different from Deignan (2006), which shows that metaphor dominates the conversion between different parts of speech of a word.

In this case, can it be claimed that the primary meaning, i.e. an enemy \& spy reporting number, is the literal meaning as declared by the traditional view of meaning? Or is it the prototypical meaning as announced by the classic prototype view? Or is it the central meaning as claimed by the abstractivist view? Internalized categories do not exist alone, but rather they combine and extend in actual use. In this sense, categorization is actually endless re-categorization. Different categorizations result in different concepts and prototypes. Concepts do not stand alone, but are instead related to each other; one concept can be established on other concepts, see also Carroll (1964), and one concept develops to another concept. If police emergency is the central meaning and the prototype from the current uses of " 110 " in the corpus, then a question may arise: Is this prototype the same as the initial use of " $110 "$ as an enemy \& spy reporting number and as the use of a criminal reporting number? The answer is definitely not. From the above analysis, support might not be given to any of these views. Because these meanings of "110" are obtained from actual uses in authentic discourse, and the meanings are developing with the social use of " 110 " in different situations and contexts, these meaning changes in actual language use are not even be realized. Meaning change takes place on the conceptual level and results from language use, which is most of the time unconscious. In this sense, meaning change cannot be predicted; it can only be described, synchronically, and if possible, diachronically. However, meaning change is motivated by cognitive processes.

\subsection{3 "110" in China: Metaphorical projection}

The above analysis suggests that metonymy as a cognitive process motivates the extension in terms of both syntactic value and semantic value of "110" as the police emergency phone number. The above analysis also shows that police emergency is 
closely related in the cases, whether syntactically as noun, adjective, and adverb or semantically as police team, police vehicle, service centre, and service system. If this takes place within the same scenario/frame/ICM of police emergency, there is still another situation in which " 110 " is conceptually projected from the police emergency scenario to other cognitive scenarios. This is another interesting phenomenon involving " 110 " in the corpus, i.e. its extended use in fields other than police emergency-related situations. This cross-scenario projection of " 110 " is done by placing before " 110 " other nouns usually indicating walks of life such as economy, education, agriculture; for example, Power 110, Water 110, Metropolis 110, Maladministration 110 (a reportage title); or sometimes nouns indicating "being composed of" such as Party members 110, Volunteers 110; or sometimes nouns indicating "in the way of", e.g. Texting 110, to fulfil a modification function thus projecting it to other fields, indicating "vehicle", "team", "system", "centre", "phone number", and "service" involved. It indicates that police emergency is the default of "110", others are all extended or marked cases. The following are examples from the Internet-ZH corpus:

(66) 黑夜里, 风雨中, 电力 110 风驰电掣于大街小巷、乡村田野。 (vehicle of the electric power company)

Hei1 ye4 li3, feng1 yu3 zhong1, dian4 li4 110 feng1 chi2 dian4 che4 yu2 da4 jie1 xiao3 xiang4, xiang1 cun1 tian2 ye3

In the wet and windy night, the Electric Power 110 dashed through the streets and lanes, across the fields in the countryside.

(67) 老百姓感激地称赞: “你们电力 110 真是太神速了, 我们才刚刚起 床...... (working team of the electric power company )

Lao3 bai3 xing4 gan3 ji1 di4 chen1 zan4: Ni3 men2 dian4 li4 110 zhen1 shi4 tai4 shen2 su4 le, wo3 men2 cai2 gang1 gang1 qi3 chuang2

The folks commended with gratitude: You (Electric Power 110) are amazingly quick, we are just up. 
(68) 广州将开通短信 110 平台市民发短信也可捉贼。（mobile texting system)

Guang3 zhou1 jiang1 kai1 tong1 duan3 xin4 110 ping2 tai2 shi4 min2 fa1 duan3 xin4 ye3 ke3 zhuo2 zei2

In Guangzhou a Texting 110 system will be established and the residents can report thefts to the police by texting.

(69) 后电视台做了一台《东方 110》节目，...... (police (team) program)

Hou4 dian4 shi4 tai2 zuo4 le yi1 tai2 dong1 fang1 110 jie2 mu4

Later the TV station made a program called "Oriental 110"

(70) 可以打 $0797 * * * * * * *$, 这是我们和谐 110 的电话, 24 小时有人 值班。(centre)

Ke3 yi3 da3 $0797 \ldots$, zhe4 shi4 wo3 men2 he2 xie2 110 de dian4 hua4, 24 xiao3 shi2 you3 ren2 zhi2 ban1

You can dial $0797 \ldots . .$. , this is the telephone number of Harmony 110, and it is watched 24 hours a day.

(71) 情急之下, 李勤拨打了校园 110 让请求调查。(phone number)

Qing2 ji2 zhi1 xia4, li3 qin2 bo2 da3 le xiao4 yuan2 110 rang4 qing3 qiu2 diao4 cha2

Under desperation, Li Qin dialled the phone number of the Campus 110 and asked for an investigation.

(72) 到学校公安处各校园 110 值勤点或就近的地方公安机关报案 (service)

Dao4 xue2 xiao4 gong1 an1 chu4 ge4 xiao4 yuan2 110 zhi2 qin2 dian3 huo4 jiu4 jin4 de di4 fang1 gong1 an1 ji1 guan1 bao4 an4 
Report to the service loci of the Campus 110 established by the public security department of the university or report to the nearest local public security organs.

The Internet-ZH corpus shows 45 hits for such "110" modified by other nouns, of which 27 are related to the "police" scenario, indicating police, police service, and police reporting system. For example:

(73) 各级公安经侦部门充分发挥经侦“110”协作机制的作用, 在元旦春 节 (system)

Ge4 ji2 gong1 an1 jing1 zhen1 bu4 men2 chong1 fen1 fa1 hui1 jing1 zhen1 110 xie3 zuo2 ji1 zhi4 de zuo4 yong4, zai4 yuan2 dan4 chun1 jie2

The economic investigation sections of the public security organs at all levels should bring into full play the Economic Investigation 110 as the coordination mechanism, during the New Year and the Spring Festival...

(74) ......实离我们很近网上“110”......：(police)

Qi2 shi2 li2 wo3 men2 hen3 jin4 wang3 shang4 110

...Actually very close to us the Internet $110 \ldots$

(75) 先后成立了“反家庭暴力 110 报案中心” 和 ...... (service)

Xian1 hou4 cheng2 li4 le fan3 jia1 ting2 bao4 li4 110 bao4 an4 zhong1 xin1 he2

Anti family violence 110 reporting centre and ... were established in succession

Accordingly, a search of the People Website with news headline containing "110" 56 found much more such extended use, involving almost every aspect of life, such as Municipal Administration and Development, Environment, Charity, Agriculture, Tourism, Education, Science, Seniors Care, Laws (Lawyer 110), Weather, Vehicle

\footnotetext{
${ }^{56} \mathrm{http}: / /$ search.people.com.cn/rmw/GB/rmwsearch/gj_search_pd.jsp, retrieved on 20 Nov, 2009. This website is an official governmental news site of China run by the People's Daily and People's Daily Online since 1997. The corresponding English website is http://english.peopledaily.com.cn/
} 
/Transport, Foodstuff, Telecommunication, Politics, Economy, Banking, Medical, Examination, Psychology, etc. Please see Appendix 1 for a detailed list.

The cases found in the People Website data can be roughly divided into three classes, the first is police-related service/team, not necessarily involving emergency situations, and the phone number concerned does not even involve the number "110", though the greeting term contains “110”; e.g. hai3lu4 海路 110 (Sea and Land 110 (police on both land and sea)), hai3shang4 海上 110 (Sea 110 (police on sea)), di2shi4 的士 110 (Taxi 110) (the taxi drivers act as clue and help provider to the police). Except the last one, all these have the same function as the default police emergency situation, the difference is that they apply only to specific aspects concerned. The second class is governmental service excluding police-related field, such as tong2du1yan2ye4 铜 都 盐业 110 (Copper Urban Salt 110), shi2pin3an1quan2 食品安全 110 (Food Safe 110). The third class is nongovernmental, including both commercial and non-commercial services, e.g. dian4xin4 电信 110 (Telecom 110), wen2hua4qiang2guo1 文化强国 110 (Cultural Power 110), zheng4quan4 证券 110 (Stock Market 110), etc. The second and third classes are the most dominant in current China. However, "110" in all these are just labels; the emergency phone numbers for the services do not even contain the three digits "110", and the situation is not really an emergent one at all. Most of the extended use of " 110 " is positive and free of charge for the service provided, especially in the government services. But there are also some examples that are negative and even illegal, charging fees for the service provided, e.g. di4xia4 地下 110 (Underground 110), which charges fees for helping one side settle disputes illegally by fighting and frightening the other side; and min2jian1 民间 110 (Nongovernment 110, a non-police security service), which also charges for the service of maintaining the order of stores, enterprises, entertainment places and solving some related problems, and of patrolling around.

The police-related class is concerned with accepting police-related (emergency) reporting and providing timely police help in emergency situations. The second governmental class is concerned with both providing help in need and accepting reporting of some maladministration or illegal performance in the government 
services. The last class is both accepting informative or fault reporting such as News 110, Telecom 110, and providing help or information in need such as Majhong 110, Automobile Fitting 110, Stock Market 110. For instance, Majhong is nowadays played in the cities on electronic Majhong tables, which allow automatic resetting after each round and therefore much less time wasting; if the electronic Majhong table does not work well, you can dial "Majhong 110" service number (nothing to do with "110"), and they will come to help fix it with a service charge.

From the police-related class to governmental (excluding police) and nongovernmental classes, "110" is motivated by metaphoric cognitive processes. The meaning prototype is also developed. The meaning prototype of POLICE EMERGENCY in the police-related field develops to TIMELY ASSISTANCE and INFORMATION REPORTING, which are actually part of the central knowledge of the POLICE EMERGENCY category. In the POLICE EMERGENCY scenario, it can be easily figured out that it involves reporting the emergency situation by dialling the police emergency number " 110 ", the police then immediately come to the spot to offer help; because it is an emergency for police, police emergency thus becomes the prominent figure of the central knowledge with timely help and information reporting in the ground. It is POLICE that limits both POLICE EMERGENCY's use and extension. Because the function of " 110 " as police emergency number was gradually intensified in actual social use as mentioned in Section 4.1, the other aspects of POLICE EMERGENCY such as INFORMATION REPORTING and TIMELY ASSISTANCE pop up to dominant positions and become two kinds of central knowledge and thus two meaning prototypes, which pave the way for the metaphoric projection of " 110 " to other fields. This is probably what Kövecses means by saying that only a small part of the community-shared central knowledge of the source is inherited by the target in metaphor. So it seems that it is in essence the metonymic process of INFORMATION REPORTING FOR "110" and TIMELY ASSISTANCE FOR "110" that provides a conceptual prerequisite for metaphoric projection of " 110 " respectively to the governmental (with police excluded) and the non-governmental classes.

The findings in the Internet-ZH corpus and People Website show that "110" has been in use far beyond the previous police emergency situation, not to mention the 
initial enemy and spy reporting situation. "110" has also developed from the initial enemy and spy reporting phone number to criminal reporting number, to police emergency number, to special labels of emergency service, to special labels of service in need. In the latter two situations, "110" does not even appear in the service phone number, it functions only as a symbol. The meanings of " 110 " in actual contexts are far from exhaustive, as shown in the corpus; it goes from actual number to superficial label, develops from the original numerical meaning to variable meanings related in different situations.

It thus suggests that the development of " 110 " in use has undergone several "generations", and the meaning prototype of " 110 " is functionally dynamic. The original category of " 110 " is the category of number; when it is used as a special phone number, it moves to special categories. For example, it moves to the military category when it is used as enemy and spy reporting phone number (which reflected the situation in those days); it moves to the police category when it is used as criminal and police emergency phone number; and it moves to other variant categories when it is extended to fields other than the police. When " 110 " is used as a special phone number, it is (re-)conceptualized and (re-)categorized within the specific scenario with a different meaning prototype. For example, as the enemy and spy reporting phone number, " $110 "$ is conceptualized as hostility-related entity; as the police emergency phone number, "110" is conceptualized as police-related entity; as the information reporting number, " $110 "$ is conceptualized as information-related entity; as the assistance phone number, " 110 " is conceptualized as assistance-related entity. Thus in different scenarios of use, "110" bears different meaning prototypes. The meaning prototype of NUMBER, the meaning prototype of ENEMY, the meaning prototype of POLICE, the meaning prototype of EMERGENCY, the meaning prototype of INFORMATION, and the meaning prototype of ASSISTANCE represent the dynamic development of " 110 " in terms of meaning prototype. All these can be encompassed in the conception of " 110 " and internalized in the conceptualization of " 110 ". The public who do not know of the development of " 110 " conceptualize " 110 " only as police emergency phone number and generate the meaning prototype of POLICE, but with the extensive use of " 110 " in other fields or scenarios, they will gradually (re)conceptualize "110" as an assistance 
phone number, generating the meaning prototype of ASSISTANCE which has nothing to do with the police and even with emergency situations.

It is obvious in the corpus and People Website that "110" has been extended from the originally adopted special enemy and spy reporting phone number and criminal reporting phone number to the current police emergency phone number and the simple symbol of emergency and assistance. It embraces the category of NUMBER, the category of PHONE, the category of POLICE, the category of EMERGENCY, the category of INFORMATION, and the category of ASSISTANCE. Each category forms its own central knowledge of the meaning prototype. It covers noun, adjective and adverb in terms of syntactic value and comprehends phone number, service system, team, individual, vehicle, service centre, and notice/call from the service centre and so on in terms of semantic value. It is hard to declare that there is a basic/core/primary meaning shared by all these uses; it is also impossible to announce that there is a prototypical meaning among these uses, as analysed above. This is consistent with the cognitive and psychological result of concept formation and development. Concept formation tends to develop from the abstract to the more concrete and detailed, that is, initially associative generalizations are not controlled by perceptually obvious features but rather by global (super-ordinate) categories (Mandler, 1999). Thus the earliest meanings are themselves general/global, relational (Mandler, 1996). As regards "110", the initial use of enemy and spy reporting phone number has already covered the knowledge of PHONE, EMERGENCY, POLICE, INFORMATION, ASSISTANCE, TIMELY, etc.; with each experience increment, the abstract meaning develops and gradually becomes specific in specific scenarios, and certain knowledge comes to the fore and ascends to the meaning prototype status .

In the dynamic development of word meaning, both the structure of meaning and its psychological nature change; "it is not merely the content of a word that changes, but the way in which reality is generalized and reflected in a word" (Vygotsky, 1986, pp. 121-122). Meaning prototype is dynamic and developmental, for concept formation itself is a dynamic and developmental process. Concept as such is functional, it derives from "attentive analysis" (Mandler, 1996, p. 372), which brings, in the union of meaning and form, the first concepts (Mandler, 1998b). 


\subsection{Summary}

Meaning is the value of a word for its existence and development. Word meaning originates from the functional generalization of the experience of reality, and it is an internalized reflection of the experience. Meaning is thus in essence conceptual. It is borne in words as forms. Word meaning therefore belongs simultaneously to both language and thought, and to both linguistics and psychology. Language is part of the human cognitive faculty; it derives from human cognitive activities. Word meaning can thus be viewed and weighed from the perspective of cognition.

A word does not come into use with many meanings in the first place, but rather it comes into use with a conceptually global meaning that involves floating knowledge, part of which will become the central knowledge in certain situation and thus become the meaning prototype. A word thus rolls on more meanings in actual use. Meaning is in itself developmental and is a prototypical concept. Meaning change and meaning development derive from the dynamic nature of meaning prototype. Developmental meaning prototype evidences that it is wrong to propose only one prototype for a word with many meanings. Prototype itself is a prototypical concept, which means it develops. So, prototype is not static and established once and for all as the classic prototype view of word meaning claims.

Meaning as a prototypical concept can embrace different word classes and different word forms through some cognitive processes such as metonymy and metaphor. Meaning prototype is formed out of central knowledge. Knowledge is embedded in conceptualization and grows with experience, but it is the central knowledge of meaning prototype which develops in actual uses and changes with different categories in different contexts, and that determines the meaning prototype and the range the meaning prototype can reach. Thus the developing central knowledge decides the development of a meaning prototype, and meaning prototype is hence dynamic and functional.

The dynamic of meaning prototype can only be noticed by looking at the development process. In China, "110" has undergone meaning prototype development in actual uses. From the natural number to the enemy \& spy reporting phone number, to criminal reporting number, to police emergency number, to 
information reporting symbol, to assistance symbol, the meaning prototype of " 110 " has developed. The prototype proceeded from pure number to phone number and to symbol, developed from NUMBER category to PHONE category and to POLICE category, to EMERGENCY category, to INFORMATION category, and to ASSISTANCE category. Different category usage results in different central knowledge and hence different meaning prototype. This category combination extends the range of use of " 110 " as a result of central knowledge development, which brings in the continuation and breadth of its use. The central knowledge of "110" as a police number of emergency reporting and timely assistance makes it possible for it to be used in different word classes and with different semantic values. " 110 " as different word class and different semantic value in the corpus is motivated by metonymy, which involves relating to the central knowledge of the meaning prototype. The extensive use of " 110 " in fields other than the police-related is motivated by metaphor, which involves projecting some central knowledge of the meaning prototype to other categories.

As noticed, this analysis is mainly carried out by the corpus approach; more work is in need for the identification of meaning prototypes in actual language users, especially from the psycholinguistic perspective. Realizing the dynamic nature of meaning prototype also raises some questions. From the perspective of cognitive development and conceptualization, it is understood that prototype is established without any conscious awareness. The question then is how to identify the meaning prototype since it is dynamic. The suggestion would be to establish diachronic corpora and compare them in a time order, checking the degree of lexical category cohesiveness and distinctiveness achieved by the various senses (Barcelona, 2009), together with psycholinguistic experiments, as mentioned in Note 45. The second question is how, or to what extent, the global-to-specific conceptualization theory obtained from experiments in developmental psychology and cognitive psychology can be employed to describe and interpret word semantic change or other linguistic phenomena; systematic psycholinguistic experimentation is really expected. The third question is whether the dynamic theory of meaning prototype can cover nonnumber symbols in description and interpretation in terms of meaning development and meaning organization, since this chapter mainly discusses number symbol "110". Another question that arises from this chapter is why an emergency number like 
"110" can be metaphorically extended so pervasively in China when there is no such phenomenon in other countries while they all have emergency numbers. This might be viewed from other perspectives, e.g. social cognition, focusing on the terms of AUTHORITY, POWER, PRIVILEGE, which are embedded in "110" as used in the police scenario. However, all these are matters for future research. 


\section{Chapter 7 Metonymy and Football Nicknames}

The foregoing chapter examined the crucial function of metonymy in meaning change and development, especially in meaning prototype development with a case analysis of " 110 " as emergency phone number in modern China. It argued that meaning is a prototypical concept, and its prototype is dynamic and developing in use; and the development of meaning prototype is largely decided by metonymy as an innate cognitive mechanism. Metonymy seems to be more vital than metaphor in meaning prototype development which also follows the general-specific tendency. Having analyzed metonymy in such typical language phenomena as grammaticalization and meaning change, this chapter will move to examine metonymy underlying football (club team) nicknames ${ }^{57}$. Nicknaming, when viewed from the conceptual perspective, is also a cognitive process like grammaticalization. If grammaticalization can be said to be of language as a system, nicknaming would be of language as a product. This chapter considers football nicknames as a cognitive product, arguing that football nicknames represent the coiners' conceptualization and categorization in a specific functional perspective. It then examines the cognitive motivation for football nicknames, identifies the patterns of football nicknames, and the underlying cognitive mechanisms.

\subsection{Introduction}

Nicknames are studied mainly as a social phenomenon from the socio-cultural or anthropological perspective in the field where they apply to people. For instance, Morgan et al. (1979) make a specific study of nickname use amongst school children from the perspective of social education, trying to figure out why school children invent nicknames and how nicknames influence the character and personality development of school children, i.e. the social and psychological power of nicknames. Nicknames in sports are also touched upon, but they are mostly lists of nicknames of professional sports players, e.g. in baseball, football, basketball, hockey, or they are referred to in funny stories about how they came to be attributed to the players concerned (see Pruyne, 2002). However, the cognitive aspects of

\footnotetext{
${ }^{57}$ Football nicknames in this research are exclusively short for the nicknames of football club teams. For the sake of convenience, only "football nicknames" are used.
} 
nicknames, regardless of whether they apply to people or place or sports, have not been researched.

Nicknames are most widely used in sports (see Pruyne, 2002). Football is one of the most popular sports in England. There are over 7000 football clubs as members of a league in the English football league system. ${ }^{58}$ Almost every football team has its own nickname. This chapter will study football nicknames from the cognitive perspective, taking as a case study the 92 club teams in 2009-10 seasons Premier League and Football League (covering Football League Championship, League One, and League Two).

In Cognitive Linguistics, language originates from human mind, and the structure of language, which is considered to mirror the conception of reality, reflects the conceptual system. See e.g. Evans \& Green (2006), Geeraerts (1997), Lakoff \& Johnson (1980), Radden (1992). Besides reflection, language influences and affects human conceptualization and categorization as well (Vyvyan Evans \& Zinken, to appear). Therefore motivation and functional explanation are the key concepts of Cognitive Linguistics (Radden, 1992). In looking at football nicknames, the aims are thus: (1) To examine what motivated the birth of football nicknames; (2) to identify typical cognitive mechanisms underlying these team nicknames; (3) to analyse the function of football nicknames thus motivated.

In order to achieve the above aims, this chapter will first argue that a nickname is a conceptual product in section 7.2, and then conduct the analysis of 92 football nicknames in section 7.3, examining how people (especially the coiners) conceptualize them and identifying the underlying typical cognitive processes. Section 7.4 will discuss the distinction between nicknames of people and nicknames of football teams. And the last section will analyse the function of football nicknames, followed by a summary of the case study.

\footnotetext{
${ }^{58}$ See http://en.wikipedia.org/wiki/English_football_league_system (accessed on 25 Oct.2009)
} 


\subsection{Nickname as a conceptual product}

\subsubsection{About nicknames}

Nickname is defined in Oxford English Dictionary as:

A (usually familiar or humorous) name which is given to a person, place, etc., as a supposedly appropriate replacement for or addition to the proper name. $^{59}$

Nickname is originally from old English an ekename (an additional name), which was later (in the 15th century) mistaken as a nekename. A nickname is often regarded as an unofficial extra name mostly given to people, to places, and to all kinds of objects and creations as well (Crystal, 1992). Regarded as unofficial, nicknames are labels attached in the course of existence and are usually bestowed by peers in a variety of informal social situations (Morgan et al., 1979, p. 98).

Then it might be asked why nicknames exist and what the functions of nicknames are. Morgan et al. (1979) think that nicknames originate from children's spontaneously creative language use and function as social demarcation of specific small groups, because nicknames show that children are constructing their own autonomous social world that is independent of adult influences. Hanks (2006) points out that adults coin nicknames with the same readiness as children, and nicknames are an affective way of creating a humorous effect and promoting social interaction. However, these theorists have not provided definite answers to the questions why and how people bother to give nicknames, especially football team nicknames. This relates ultimately to the cognitive mechanisms.

\subsubsection{Cognitive perspective of nicknames}

Language is part of the human cognitive faculty, and is the outcome of general properties of cognition. Cognition is grounded in embodied experience; language is thus embedded in human experience and human cognitive behaviour (see Croft \& Cruse, 2004; Evans et al., 2007a; Evans \& Green, 2006; Geeraerts \& Cuyckens, 2007a; Lakoff, 1987b; Lakoff \& Johnson, 1980). In the cognitive perspective,

\footnotetext{
${ }^{59}$ See online Oxford English Dictionary
} 
language is considered to reflect patterns of thought, thus to study language is to study patterns of conceptualization (Evans \& Green, 2006). As the most prominent and conspicuous representation of human cognitive faculty, language inevitably reflects the embodied nature of conceptual organization and influences and affects human conceptualization and categorization (see Evans \& Zinken, to appear). The meaning of language originates from language use. Categories and structures in language are "built up from our cognition of specific utterances on specific occasions of use" (Croft \& Cruse, 2004, p. 4). Language is accordingly motivated (see Barlow \& Kemmer, 2000; Cuyckens, et al, 2003; Radden \& Panther, 2004). As Janda (2006) commented, "Though the motivations vary [...], at an abstract level, these motivations yield a consistent pattern". Language is considered as a way of organizing knowledge that reflects the needs, interests, and experiences of individuals and cultures, and linguistic meaning is thus taken as perspectival in nature (Geeraerts \& Cuyckens, 2007a). Geeraerts (2006a, p. 27) rightly points out, "Linguistic structures are thought to express conceptualizations, and conceptualization goes further than mere reference" (emphasis is mine). Therefore the study of language use is the study of the specific patterns of conceptualization and categorization of the mind on specific occasions. Vygotsky (1986) also argues that as a method of structuring personal experience internally, language can act as the initial symbol of the mental processes and determine prejudice in advance. Hence, studying how one talks to and of another can lead to what one has in mind about the talked about (Morgan et al., 1979), i.e. one's conceptualization of another, not merely reference identification, or name change, or reference shift.

Since nicknames come from language use, nicknames are both a language and a cognitive phenomenon. From the cognitive perspective, nicknames are strongly motivated. Nicknames reflect the conceptualization and categorization of the nicknamed in the nickname coiners' mind. Nicknames actually represent the coiners' cognitive perspectivization on the nicknamed. A certain nickname was given because the nickname coiner(s) tended to perceive, conceptualize, and categorize the nicknamed exactly that way due to a known demonstrated aspect that was typical of that person. That is to say, in the development of cognition, the conceptualization of an entity is functionally guided to and attentively focused on a particular salient aspect of the entity, and hence the conceptualization and categorization of the entity 
is established according to this particular salience of the entity in the social-cultural environment. This is just how cognition works, and it is metonymy that inscribes embodied cognition. As argued in Chapter 3, Embodied Cognition determines that what is perceived is only part or aspect of the entity, part about the entity, because Embodied Cognition is goal-directed, which means that attention is only paid to what is meaningful in a situation and the conceptualization and categorization is based on this particular point of view. Even so, in the conceptualization, the entity is still taken as a whole concept or category based on the salient part or aspect perceived and conceived. Nicknames work the same way and reflect such cognitive process. That is, the birth of nicknames is in nature the cognitive perspectivization in conceptualizing and categorizing the objects, they are conceptual products.

When applying to people, nicknames are surely "the symbols used with the most specific intention of categorizing people" (Morgan et al., 1979, p. 105). According to Morgan et al., nicknames can be viewed as the external manifestation of the concepts that others have of that individual, their implications, their biases, their limitations. That is, nicknames can be interpreted as indicative of the conceptual organization of those who coin the name. When applying to football teams, nicknames represent the conceptualization and categorization of the teams in the mind of the nickname coiners and hence exert great influence upon others' conceptualization of the team. That is, football nicknames are products of the nickname coiners' conceptual process, and they reflect how the teams are particularly viewed by the coiners and they also influence the fans' conceptualization of the team.

Nicknaming therefore is a conceptual activity, which involves interpreting something appropriately through a specific prominent aspect of it, at least from the perspective of the nickname coiners. As pointed out by Hanks (2006, p. 624), "Nicknames are based on a salient distinctive character or characteristic". It might be because the nicknames were intended to represent or symbolise their own peculiarity distinct from others in that or other region at the time of the name coining, which was what the coiners expected and took pride in most. Football nicknames therefore reflect how people specifically conceptualize and categorize the teams when they give the names. 


\subsection{Football nicknames: Cognitive patterns and mechanisms}

\subsubsection{Cognitive patterns in football nicknames}

As mentioned in the introduction section, the case studied involves the 92 English football teams in Premier League (20 teams), Football League Championship (24 teams), Football League One (24 teams), and Football League Two (24 teams) during the 2009-10 seasons. The reason for choosing these teams is that they form the top levels with great influence and are the most popular professional football teams in the English football league system. ${ }^{60}$ Besides the websites in footnote 58, other sources are also used to make sure of the nicknames and the origins of some nicknames, so that the cognitive patterns and cognitive processes can be as right as possible in the data analysis. For example, the research employed the information on the Football-club company official website, ${ }^{61}$ and made reference to the list of football club nicknames in the United Kingdom, ${ }^{62}$ and emails were also sent to some clubs to ensure the accuracy of the information concerning the nicknames' birth, such as Watford FC, Cheltenham Town FC, Barnet FC, Cardiff City FC, Brentford FC, and Wigan Athletic FC. For instance, The Bluebirds for Cardiff City is supposed to be based on the home kit stripe in footnotes 45 and 46, but according to the reply from the club to the email inquiry, fans formulated the nickname from the performance in a Cardiff theatre of a play called "The Blue Bird" by a Belgian author named Maurice Maeterlinck in Cardiff City's second season as a professional club (1911/12). Subsequently, a comparison and contrast analysis was conducted, and classified the nicknames according to the connection between nicknames and the club teams, as detailed in Appendix 2. Although tracing the football nicknames sounds like anecdote and legend descriptions, as a phenomenon of language use, nicknames definitely reflect the perspectivization of the nickname coiners' conceptualization and categorization of the teams. This is what is focused here on, i.e. examining the cognitive motivations underlying the somehow legendary origins and the functions contributed by those cognitive motivations.

\footnotetext{
${ }^{60}$ Most information is from the following websites: (accessed on 25 Oct.2009) http://en.wikipedia.org/wiki/English_football_league_system http://www.premierleague.com/page/Home/0,12306,00.html http://www.football-league.co.uk/page/Home/0,,10794,00.html

${ }^{61} \mathrm{See}$ http://www.the-football-club.com/football-nicknames.html (accessed on 25 Oct. 2009)

${ }^{62}$ See http://en.wikipedia.org/wiki/List_of_football_club_nicknames_in_the_United_Kingdom, (Accessed on 25 Oct. 209)
} 
In the view of Morgan et al., there are two methods of formation in all naming systems. One is the internal method, i.e. the name formation depends on the language per se, such as alliteration, contraction, suffix addition, and rhyming. The other is the external method, i.e. the genesis of the name relies on external factors of language, such as history, appearance, qualities (physical, intellectual, etc.), relationship, and local culture (Morgan et al., 1979, pp. 36-38).

Hanks (2006, pp. 625-626) thinks that the main types of nicknames may be classified as follows: Reference to physical appearance; Imputation of characteristics; Behaviour; Supposed resemblance to an animal; Cultural references; Punning; Reference to a job or social function; Pun on surname; Rhyming and assonance; Survival of baby talk.

What Hanks and Morgan et al. are concerned with are nicknames of people. But football nicknames in this study are club teams, not exactly a specific person, rather a whole group as a functional collective, excluding the fans who call themselves the nicknames of the team(s) they support. Therefore a fresh analysis of the football nicknames is needed to see what categories or classifications can be imputed from the relation between the nicknames and the football teams and what distinctions can be made between individual person nicknaming and football team nicknaming.

The collection of data (see Appendix 2 for the collection) shows that some teams have more than one nickname, e.g. "Ipswich Town" has three nicknames: "Blues", "Town", "The Tractor Boys". In such a case, the nickname appearing on the official website of the league they belong to is chosen. For example, in case of "Ipswich Town", the selection is made to the nickname that appeared on the official website of the Football League Championship to which it belongs, i.e. "The Tractor Boys". It is also found that some teams have two nicknames on the official website of the League they belong to. In such cases, both of the nicknames are taken into account, and the underlying cognitive mechanisms are examined respectively. Therefore though the case study only contains 92 club teams, there are 97 nicknames to analyse, for there are five teams which have two nicknames on the official website. But the analysis does not pay attention to which one is more commonly used in actual reports or discourse, because the focus is the underlying cognitive motivations. The 
analysis and statistics suggests that there are obviously some main connection types between the nicknames and the club teams, as shown in the following table.

\begin{tabular}{|c|c|c|c|c|c|c|}
\hline \multicolumn{2}{|c|}{ Connection base } & \multicolumn{2}{|c|}{ Number } & \multicolumn{2}{|c|}{ Percentage (\%) } & method \\
\hline \multirow{4}{*}{$\begin{array}{c}\text { Football Club } \\
\text { (team) Name } \\
\text { Related }\end{array}$} & Initial name & 3 & \multirow{4}{*}{25} & 3.09 & \multirow{4}{*}{25.77} & \multirow{4}{*}{ internal } \\
\hline & Pronunciation & 1 & & 1.03 & & \\
\hline & Shortened name & 16 & & 16.50 & & \\
\hline & Derived name & 5 & & 5.15 & & \\
\hline \multirow{2}{*}{$\begin{array}{l}\text { Home Kit } \\
\text { Colour } \\
\text { Related }\end{array}$} & Colour of home kit & 11 & \multirow[b]{2}{*}{19} & 11.34 & \multirow[b]{2}{*}{19.59} & \multirow{5}{*}{ external } \\
\hline & $\begin{array}{l}\text { Based on home kit stripe } \\
\text { (pattern/style) }\end{array}$ & 8 & & 8.25 & & \\
\hline \multirow{3}{*}{$\begin{array}{c}\text { Local } \\
\text { Particularity } \\
\text { Related }\end{array}$} & Local history/legend & 19 & \multirow{3}{*}{52} & 19.59 & \multirow{3}{*}{53.61} & \\
\hline & $\begin{array}{l}\text { Local (traditional) } \\
\text { industry/profession/business }\end{array}$ & 20 & & 20.62 & & \\
\hline & Local place/Ground & 13 & & 13.40 & & \\
\hline
\end{tabular}

Table 7.1: The patterns in football team nicknames

The exception is Crystal Palace. The nickname "The Eagles" for Crystal Palace is usually understood to refer to the eagle featured on the club badge since 1973. The connection is unclear. But according to Ian King (personal communication), who is a historian for Crystal Palace FC, "The Eagles" was nicknamed after "Benfica" in Portugal in 1973 by Manager Malcolm Allison, who wanted to alter the image of the club.

The above diagram shows that the main cognitive patterns in forming football team nicknames in percentage from high to low are Local Particularity Related (53.61\%), Team Name Related (25.77\%), and Home Kit Colour Related (19.59\%). That is to say, football nicknames derive from these three main sources, with local relevance as the prime pattern, which takes up over half of the number. This indicates that football teams are strongly location-oriented and football nicknames are kind of reconceptualization and re-construction of local particularities. However, from the cognitive perspective, these patterns of football nicknames are in turn motivated by some cognitive mechanisms, i.e. the reason why people can form such nickname 
patterns is because of some underlying conceptual mechanisms. This point will be explicated in next section.

In terms of the naming methods proposed by Morgan et al., the external method is more popular than the internal method, taking over $70 \%$ of the whole. Actually, except Initial Names and Shortened Names, which involve the name per se and can be said to be more the result of the language internal method, the rest are all language-externally derived, because the only nickname derived from Pronunciation is "The Bees" for Brentford, which involves the misinterpretation of a terrace chant "Buck up Bs"; and the Derived Names are based on a variation of part of the nickname, specifically to form the sense of people of the club. The follow sections examine what cognitive mechanisms underlie and motivate these patterns.

\subsubsection{Local Particularity Related (LPR)}

Since Local Particularity Related, which indicates that the football nickname is related to some local particular aspect, is the most popular source of football nicknames, which is taken up as the first analysis. The local particularities consist of Local Historical or Legendary Stories of the club or the area the club is based in. For example: West Bromwich Albion's nickname is "The Baggies", which derives from "bagmen" who carried the match-day takings from the turnstiles to the cash office on the halfway line. The nickname "The Posh" of Peterborough United originates from former manager Pat Tirrel's comment that he was looking for, "Posh players for a posh team". Darlington is called "The Quakers" because of the religious movement (Religious Society of Friends, called Quakers) which had a historic influence on the town. Bolton Wanderers is called "The Trotters", because in the 19th century, one of their pitches was adjacent to a piggery and clearances often ended up in there, and players had to trot through the pigpens to retrieve the balls. So in this class the nicknames are bound with the history of the clubs and the legends or anecdotes related to them and the town they are in. Another three instances that are classified in this pattern and that are worth noting are Portsmouth, Barnsley, and Southampton with the nicknames "Pompey", "The Tykes", and "The Saints" respectively. "Pompey" is from the historical nickname of the city of Portsmouth. "The Tykes" is from the local colloquial name for people of Yorkshire where it is located. "The Saints" is from the original name of Southampton known as Southampton St. Mary's. 
This class also applies to "The Bluebirds" for Cardiff City as mentioned earlier. Therefore this sub-pattern of nickname can be roughly labelled as LOCAL STORIES RELATING CLUB FOR CLUB TEAM.

The other two in the Local Particularities Related pattern are Local Place/Ground, and Local (traditional) Industry/Profession/Business which is the most prominent of all the connection bases. The Local (traditional) Industry/Profession/Business class is composed of four main source types. The first is from traditional industry/profession or manufacture, such as "The Blades" for Sheffield United, socalled because the blade is a well-known manufactured product in Sheffield, which has been a major centre for steel production and cutlery manufacture since the 18th century. "The Brewers" for Burton Albion is from the local brewing industry. "The Chariboys" for Wycombe Wanderers is because High Wycombe has been a centre of the furniture (called Chariboy) production industry since the 19th century. The same applies to "The Glovers" for Yeovil Town, "The Bobblers" for Northampton Town, "The Gunners" for Arsenal, "The Hatters" for Stockport County, "The Iron" for Scunthorpe United, "The Potters" for Stoke City, "The Saddlers" for Walsall. The second type is traditional animal breeding of the area. For example, "The Bulls" for Hereford United is named after the local breed of cattle. The same applies to "The Canaries" for Norwich City and "The Terriers" for Huddersfield Town. The third is traditional heritage of hunting or agriculture or fishery. For example, The Foxes for Leicester City refers to Leicestershire's hunting tradition. ${ }^{63}$ "The Tractor Boys" for Ipswich Town refers to Ipswich's agricultural heritage. The same applies to "The Shrimps" for Morecambe, "The Shrimpers" for Southend United, and "The Silkmen" for Macclesfield Town. The fourth is from local commercial shops/business. For instance, the nickname Everton is nicknamed "The Toffee" after a local shop which sold sweets, including the "Everton Mint", on match days. "The Addicks" for Charlton Athletic is a corruption of the word "Haddocks", named after a local fishmonger. Therefore this sub-pattern can be generally labelled as LOCAL BUSINESS RELATING CLUB FOR CLUB TEAM.

\footnotetext{
${ }^{63}$ Another coincidence is that the outline of Leicestershire on a map resembles a fox's head; if taken as this, then it is a combination of metonymy and metaphor, which will be described below.
} 
In the Local Place/Ground class, the name of a particular place/object in the city/town or a particular place adjacent to the team ground or the name of the area where the club is based is used for the club team. For example, "The Millers" for Rotherham United, "The Owls" for Sheffield Wednesday, and "The Cottagers" for Fulham are all based on the home ground of the team. "The Bees" for Barnet comes from the location of apiaries close to the Underhill ground in the early years of the 20th century. "The Black Cats" for Sunderland, "The Cumbrians" for Carlisle United, "The Railwaymen" for Crewe Alexandra, "The Royals" for Reading, and "The Spireites" for Chesterfield are derived from the name of the town/county or the name of a specific object or place in the city: "The Spireites" for Chesterfield is nicknamed for the famous crooked spire on the local Church of St. Mary and All Saints; "The Black Cats" for Sunderland is named after the Black Cat Battery gun based on the River Wear. Some other nicknames are based on the local link to seaside or seaport, such as "The Gulls" for Torquay United, "The Seagulls" for Brighton \& Hove Albion, and "The Seasiders" for Blackpool, so derived from their local link to the seaside. What is interesting is that "The Seagulls" for Brighton \& Hove Albion is also a response to the similar sounding nickname "Eagles" of their arch rivals Crystal Palace, which also indicates that team nicknames are taken very seriously. From the above examples, this sub-pattern may be approximately labelled LOCAL PLACE/GROUND RELATING CLUB FOR CLUB TEAM.

\subsubsection{Football Club Name Related (FCNR)}

Following Local Particularity Related in percentage is the Football Club Name Related (FCNR) pattern, which is much less than LPR. The diagram shows that in this pattern, the subclass of shortened names, which is the shortened name of the football club, is the largest. For instance, "Boro" for Middlesbrough, "The Dale" for Rochdale, "The Gills" for Gillingham, "The Latics" for Oldham Athletic and for Wigan Athletic, "The Rovers" for Blackburn Rovers and for Doncaster Rovers and for Tranmere Rovers, "The Shrews" for Shrewsbury Town, "The Shots" for Aldershot Town, "Stanley" for Accrington Stanley, "United” for Leeds United, "The Swans" for Swansea City, "Wolves" for Wolverhampton Wanderers, and "Pools" for

\footnotetext{
${ }^{64}$ The Millers was named after the club's home ground (Millmoor); the Owls was based on the name of the area in which the club is based (Owlerton); the Cottagers was from the club's home ground, Craven Cottage, see Appendix 2 for details.
} 
Hartlepool United, which refers to the twin towns of Hartlepool and West Hartlepool prior to the integration of the two in 1967. From the above it is clear that shortened names are actually motivated. The shortened names are adopted as a special case with particular meaning or implication. The word shortened for the nickname is often more meaningful and sounds intimate in the club name. The shortened names of the club names as nicknames are not taken casually or arbitrarily but rather with care and intention or expectation. When shortened names are selected as the nicknames, they bear some sense. That is, a certain meaning (intention) is vested in the club team together with the nickname. The point will be discussed further in section 7.5. This feature applies to Derived nicknames as well.

As mentioned earlier, the Derived names are a variation of a selected part of the team name, and this part is usually easy or likely to form a meaningful name with the sense of people. For example, "The Citizens" for Manchester City, "The Daggers" for Dagenham \& Redbridge, "The Dons" for Milton Keynes Dons, "The Valiants" for Port Vale, and "The Villans" for Aston Villa. For initial letter as nicknames, such as "The O's" for Leyton Orient, “The R's" for Queens Park Rangers, and "The U's" for Colchester United, the tendency is also that the one that bears strong sense or intention or is more meaningful in the team name is more likely to be the nickname source. Regarding "The Bees" for Brentford, the nickname "The Bees" is a misinterpretation of an early terrace chant "Buck up Bs"; however, it could also come indirectly from the initial letter of Brentford. So with equal motivation as LPR, this pattern can labelled in general as VARIANT OF CLUB NAME FOR CLUB TEAM

\subsubsection{Home Kit Colour Related (HKCR)}

This pattern consists of two classes. One is "Colour of home kit", i.e. the nicknames of the club teams are exactly the colour names of their home kit, mostly the colour of the shirt, and the home kit of these teams is usually single-coloured. For example, "The Blues" for Birmingham City and Chelsea and Manchester City, "Th "The Clarets"

\footnotetext{
${ }^{65}$ What is interesting is that there are some teams who share the same nicknames due to the same home kit colour or similar home kit stripe. This means that the underlying cognitive process is the same in the aspect of home kit colour/stripe. Although when they play against each other, they show difference in wearing home kit and wearing away kit, yet how the sports commentators or the sports reports talk about them is also interesting and worth further study.
} 
for Burnley, "(The) Lilywhites” for Tottenham Hotspur and Preston North End, "The Reds" for Liverpool and Nottingham Forest, "The Sky Blues" for Coventry City. The other is "Based on the colour of home kit", i.e. the nicknames of the club teams are derived from the pattern that the home kit coloured stripes (especially the shirt) forms or from the style that is formed by the colours of the home kit shirt and the shorts combined. The home kit of these teams is mostly double-coloured. The following are some instances:

"The Bantams" for Bradford City is based on the claret and amber colours of the club's home kit, which looks like the stripes of a bantam.

"The Hornets" for Watford is based on the yellow and black home kit, which looks like the stripes of a hornet.

"The Magpies" for Newcastle United and Notts County is based on the black and white colour of home kit, which looks like the stripes of a magpie.

"The Tigers" for Hull City is based on the amber and black home kit, which looks like the stripes of a tiger.

"The Robins" for Bristol City, Cheltenham Town, and Swindon Town is based on the red home kit similar in colour to a robin's chest.

There is one special case classified in the first home kit category. It is "The Red Devils" for Manchester United. This nickname is related to the home kit colour, but it carries more, i.e. "Devils". So it may also be located into the second class. Nevertheless, it is quite different from the second. "Devils" as a metaphor here is not based merely on the home kit colour stripe or pattern like the second class. It has extended to something else, e.g. the vigour the team bears, or the imposing manner the team demonstrates on the field. The second class is different from the first in that the first class is drawn directly from the home kit colour such as "The Reds", "The Blues", i.e. they are exactly the colour names of the home kit; but the second class goes further beyond the colour name proper, it is based on the observers' perception of the coloured stripes or patterns the home kit forms, such as some birds or animals with similar stripes or patterns, i.e. it is based on some similarity created by the 
viewers between the home kit stripes or pattern thus formed and the colours of some animals conceptualized previously. This is a very special case. It seems that a combination of colour stripes, perception and imagination, i.e. the nickname coiners' conceptualization and categorization, matters here. This point will be elucidated in section 7.4.

\subsubsection{Cognitive mechanisms underlying the patterns}

With more generalization and at a higher level, the processes of the football nicknames formation are roughly three kinds. One is derived from the local particularities comprising the local history/legend, the local place/ground, the local business/profession/industry, and the club team itself such as the home kit, and official names. The second is derived further from the particularities (such as the above) of the football club teams, especially their home kit colour/stripe, with a special case of "The Red Devils" for Manchester United. The last is the exception Crystal Palace, nicknamed after Benfica whose nickname is "Eagles", where there is nothing special related to the football team or club per se.

The nicknames formed in the first kind include LOCAL PARITICULARITY RELATED, FOOTBALL CLUB/TEAM NAME RELATED, and COLOUR OF HOME KIT, which are formed through the PARTICULARITY RELATING CLUB TEAM FOR CLUB TEAM conceptual process. That is to say, something that the football club is related to is used to symbol the football club and then to symbol the football club team. They are overall motivated by metonymy, so it might be said that metonymy underlies the first kind of football nicknames.

For instance, Shortened Name and Initial Name as sub-patterns are obviously ellipsis, which is licensed by metonymy due to the conventionalized knowledge of them at least to those local fans, or for other functional reasons. But the process underlying this ellipsis is liable to be metonymy mechanism, e.g. PART FOR WHOLE. The Derived name sub-pattern is also in general based on the PART FOR WHOLE process with a "people" sense addition in the PART such as "The Citizens" for Manchester City, "The Daggers" for Dagenham \& Redbridge, "The Valiants" for Port Vale, "The Villans" for Aston Villa. This is a very simple and obvious case; it is a somewhat general analysis. However, a more detailed analysis will discover that it 
is actually chained metonymy (Barcelona, 2005a) that operates in other cases. For example, in the LOCAL STORIES RELATING CLUB FOR CLUB TEAM, it is not the whole stories that function as a source of the metonymy, but only certain aspects (properties, entities, etc) of the local stories and legends that function effectively and efficiently as the reference points. This sense can be made through some examples. In "Quakers" for Darlington, Quakers as the name of the religious society is used to represent the historic movement, which is then used to represent the influence it exerted upon the town, which then is used to represent the town. This process involves several PART FOR WHOLE metonymies, i.e. they form a chain of PART FOR WHOLE metonymies. And then the town is used to stand for the football team/club in the town through WHOLE FOR PART metonymy. Thus "Quaker" for Darlington derives from a chained metonymy. By the same token, in "Chariboys" for Wycombe Wanderers, Chariboys as a furniture brand is used to stand for the furniture, and then is used to stand for the furniture production industry in Wycombe, and then this industry is used to represent Wycombe, which forms a metonymic chain of PART FOR WHOLE, and then Wycombe is used to stand for the regional football team in Wycombe through WHOLE FOR PART metonymy, thus "Chariboys" is finally used to represent Wycombe Wanderers via chained metonymies. In "Spireites" for Chesterfield, "Spireites" as the name of the famous crooked spire on the local Church of St. Mary and All Saints is used to represent the town Chesterfield (PART FOR WHOLE), then is used to stand for the football team in the town (WHOLE FOR PART). The above analysis suggests that this kind utilises a peculiarly dominant aspect of the town or the club to stand for the team in the town, which forms a PART FOR WHOLE - WHOLE FOR PART metonymic chain, a special process (PART-WHOLE RELATING) of metonymy as a cognitive mechanism. In the first PART FOR WHOLE chain, it is generally some salient aspect of the town to represent the town; in the latter WHOLE FOR PART, it is usually the town to represent the football team.

Another variant form of metonymy is double metonymy (Ruiz de Mendoza \& Díez Velasco, 2002) ${ }^{66}$, which takes place in the second home kit category, i.e. Home Kit Colour Based. The nicknames of the football teams are derived from the pattern of

\footnotetext{
${ }^{66}$ Double metonymy in this research is different from that used in Ruiz de Mendoza and Diez Velasco (2002). Their view of double metonymy is actually what I term as chained metonymy, sees pp512518 for details of their explication.
} 
the home kit coloured stripes. This derivation involves a series of cognitive activities. On the one hand, the specific striped colours together represent the home kit, the home kit stands for the team (chained metonymy); on the other hand, the specific striped colours of an animal is used for the animal. If the striped colours are placed in the middle (see diagram over page), then it goes both backward and forward. Going backward, the striped colours are for the animal; going forward, the striped colours are for the home kit then for the team, thus forming a continuum with the animal at the outset and the team at the end and striped colours in the middle. The inference involved here goes like this: The colour stripes for the home kit and then for the team, the colour stripes for the animal, thus the animal for the team. For instance, in "The Tigers" for Hull City, on the one hand, the amber and black home kit colour stripe is specifically the colour stripe of a tiger, so the amber and black stripe is for tiger; on the other hand, the amber and black stripe is the colour pattern of the home kit, thus is for the home kit, and the home kit of the team is used for the team, then the amber and black stripe is for the team. They therefore form a double metonymy, based on the common coloured stripe of the animal and the home kit. This process, unlike the chained metonymy which is an unbroken line or unidirectional, involves two directions, i.e. two independent metonymic processes. Although there is some common aspect between the animal and the team home kit, it seems that metaphor does not motivate this pattern. However, that the cognitive process in motivating the nickname is not metaphoric does not mean that the nickname cannot evoke a metaphorical mental image in the mind of the fans when it comes to their consciousness. The metaphorical effect resulting from these nicknames will be further discussed in section 7.5. Though metaphor is commonly regarded to be based on similarity, similarity does not necessarily involve a metaphoric process, as pointed by Warren (1995). The striped colour of the shirt is metonymical for an animal, because this colour is inherently a feature (part) of the animal in general, with no intention mean that this shirt or the person wearing this shirt is (like) that animal, though the colour would evoke the general knowledge of the animal. Thus the cognitive process underlying the second kind is overall still metonymy. The formed process can be illustrated as follows:

Animal $\rightleftarrows$ coloured stripe $\longrightarrow$ home kit $\rightarrow$ team 
Now is the special case of the second kind, i.e. "The Red Devils" for Manchester United. As already mentioned earlier, this nickname is based on the home kit colour of the team, i.e. "Red", but it adds some other element, i.e. "Devils". As for the home kit colour, this is definitely motivated by a metonymic process. It is derived from THE COLOUR OF HOME KIT FOR THE HOME KIT FOR THE TEAM, i.e. a chained PART FOR WHOLE metonymy. In order to make sense of "Devils", it is essential to find out the origin of this nickname. According to the source located, ${ }^{67}$ the nickname "The Red Devils" originally belonged to a nearby rugby league team Salford, the official full name of which is Salford City Reds. This is a professional rugby league club based in Salford in Greater Manchester. This nickname was earned during their tour to France, where the French press labelled them as playing like devils, i.e. the name Les Diables Rouges, is translated in English as the Red Devils. Later on, this nickname was adopted by Manchester United in the early 1960s after Manager Matt Busby heard it in reference to the red-shirted Salford rugby team, and liked the sound of it, and thought that a nasty devil is more intimidating to opponents than angelic babes. ${ }^{68}$ As analyzed earlier, in "The Red Devils", "Red" derives from the red home kit, and "Devils" originally derives from the manner of the (Salford rugby) team players on the field: They played like devils. But when it is employed to label Manchester United, "Devils" is not from the actual match performance, it is rather bestowed with an expectation, i.e. the manager hopes the team CAN or WILL play like "the Red Devils" Salford Rugby team who were stated to have played just like devils on the field. This actually involves a chained metaphor from the football team to the like-devils rugby team to the 'real' devils. The manager's expectation is more concerned with the vigour of the team in future matches: Intimidating or scaring opponents. Whether Devils is derived from played like devils or will play like devils, one thing is quite certain, there is something expected to be common to the team and Salford City Reds (and/ the devil) or some resemblance between the team and Salford City Reds (and/ the devil). This resemblance or commonality involves a metaphoric process in which the team is connected with and compared to "the Red Devils" Salford Rugby team (and/ the devils). Thus the underlying cognitive process motivating "The Red Devils" for

\footnotetext{
${ }^{67} \mathrm{http}: / /$ en.wikipedia.org/wiki/Manchester_United_F.C.\#cite_note-0 (Accessed on 25 Oct. 2009)

${ }^{68}$ During 1950's, Manchester United adopted a new youth policy and organized a new team in the First Division and made a great success, thus this new youth team was soon christened with the name "Busby Babes"
} 
Manchester United is a combination of metonymy and metaphor, though a chained metaphor is also involved.

One might say that not every fan of Manchester United knows this origin; they may presently just take it that it is simply because the team wear red as devils do and then they are nicknamed as "The Red Devils", maybe just like "The Tigers" for Hull City based on the colour stripe. But it is still different from the latter. Although it is possible that the current fans take the nickname as based on the home kit colour, the origin, which results from the initial conceptualization and categorization of the team, cannot be ignored (and this is what is under examination here). Football nicknames are not from what the current fans think they are but from what the team were in the nickname coiners' mind. A football nickname itself is a record of the history of the team, which exerts influence on the fans of later generations. What is focused here is (1) examining how people conceptualized and categorized the football teams when they coined the nicknames, (2) exploring what cognitive pattern/process underlay their nicknaming, (3) trying to identify the functions of these nicknames and how these functions take effect, therefore what is happening in the present fans' mind when using the nicknames for the teams is not taken into account.

So there are at least two stages in the case of "The Red Devils" for Manchester United. At the first stage, the conceptualization of the devil consists of some features, attributes or characteristics of the devil; at the second stage, the perception or conception of the team in the match, as in the case of the Salford Rugby League team, or configuration of the conception of the team in the mind as in the case of Manchester United invokes some similar aspects of the devil (in the latter case, some similarity is created), i.e. the team is treated as the devils. The conception of the devil can be used to describe a team which behaves or is expected to behave in a similar way to the devil, i.e. the previously perceived attribute or feature or function of the devil is projected onto the football team. Before the projection, the attribute or feature or function of the devil is conceptually taken as the devil proper. That is, the typical aspect of the devil is metonymically taken as the devil itself. For this reason, metonymy is the prerequisite for metaphor, as pointed out in Chapter 4, i.e. metonymy is the base of metaphor (Barcelona, 2000c, 2002b; Eco, 1979; Taylor, 1995, 2002a). 
The final analysis moves to the exception case of "The Eagles" for Crystal Palace. Like "The Red Devils" for Manchester United, "The Eagles" for Crystal Palace is also named after another sports team. Unlike the former, the nickname "The Eagles" has nothing to do with the home kit. As said earlier, in 1973, Manager Malcolm Allison wanted to alter the image of the club. From this, it is possible to infer that it was intended by the manager that the team should play just like the Portugal "Eagles" on the field. ${ }^{69}$ Indirectly he hoped his team would play as real eagles do and conceptualized his team as eagles. Thus here a metaphor chain is involved. Therefore, the cognitive process underlying this football nickname is mainly metaphor, but it is still grounded on metonymy operation, i.e. the particular feature for the team/eagles.

The above analysis suggests an improved diagram (Table 7.2) as follows:

\begin{tabular}{|c|c|c|c|c|c|c|c|c|}
\hline Pattern & Connection bas & & Nu & & Perces & e $(\%)$ & Method & Cognitive \\
\hline \multirow{10}{*}{$\begin{array}{l}\text { PARTICULARITY } \\
\text { CONCERNING } \\
\text { CLUB FOR CLUB } \\
\text { TEAM }\end{array}$} & \multirow{4}{*}{$\begin{array}{c}\text { Football Club } \\
\text { (team) Name } \\
\text { Related }\end{array}$} & Initial name & 3 & \multirow{4}{*}{25} & 3.09 & \multirow{4}{*}{25.77} & \multirow{4}{*}{ internal } & \multirow{9}{*}{ metonymy } \\
\hline & & Pronunciation & 1 & & 1.03 & & & \\
\hline & & Shortened name & 16 & & 16.50 & & & \\
\hline & & Derived name & 5 & & 5.15 & & & \\
\hline & \multirow{3}{*}{$\begin{array}{c}\text { Local } \\
\text { Particularity } \\
\text { Related }\end{array}$} & $\begin{array}{l}\text { Local } \\
\text { history/legend }\end{array}$ & 19 & \multirow{3}{*}{52} & 19.59 & \multirow{3}{*}{53.61} & \multirow{7}{*}{ external } & \\
\hline & & $\begin{array}{l}\text { Local } \\
\text { place/Ground }\end{array}$ & 13 & & 13.40 & & & \\
\hline & & $\begin{array}{l}\text { Local (traditional) } \\
\text { industry }\end{array}$ & 20 & & 20.62 & & & \\
\hline & \multirow{3}{*}{$\begin{array}{c}\text { Home Kit } \\
\text { Colour Related }\end{array}$} & Colour of home kit & 10 & \multirow{3}{*}{19} & 10.31 & \multirow[b]{2}{*}{18.56} & & \\
\hline & & $\begin{array}{l}\text { Home kit coloured } \\
\text { stripe }\end{array}$ & 8 & & 8.25 & & & \\
\hline & & $\begin{array}{l}\text { Colour of home kit } \\
\text { beyond }\end{array}$ & 1 & & 1.03 & 1.03 & & $\begin{array}{l}\text { metonymy- } \\
\text { metaphor }\end{array}$ \\
\hline Exception case & \multicolumn{2}{|l|}{ Similarity based } & 1 & 1 & 1.03 & 1.03 & & metaphor \\
\hline
\end{tabular}

Table 7.2: The cognitive processes underlying football nicknames

The above diagram shows that metonymy as a cognitive mechanism motivating football nicknames has the overwhelming superiority of a percentage of 97.94. Metaphor only takes up a percentage of 1.03 . The combination of metonymy and metaphor underlying football nicknames occupies a percentage of 1.03 as well.

\footnotetext{
${ }^{69}$ I am indebted to Derek Wallace for pointing out that the manager expected the team to play directly like the Portugal Eagles, it is not initially to play like the real eagles.
} 


\subsection{Function of football nicknames}

Besides the cognitive functions of nicknames as perspectivization, conceptualization and categorization, owing to the emotional property, the main functions of nicknames of people are sarcastic, insulting, humorous, and socially integrative (Hanks, 2006). Although nicknames of football teams carry no or less such emotional colouring, they actually arouse unifying support from the fans. As shown above, nicknames of football teams are mostly derived from particularities of the local region, this can make the local fans or even the local inhabitants realize that the football team is an indispensable part of the region they live in and part of their daily life. The football nickname hence functions as the regional identity and evokes a rallying power in the supporters. Support from the fans is crucial to any football team, and thus there is a dramatic difference between home play and away play. Particularity as the source of a football nickname can establish a strong sense of regional identity for the team, make the region take sides with her all the time, and finally win overwhelming power over opposing teams. This point can be illustrated in examples of Shortened names and Derived names, in which all the nicknames carry a sense of people, i.e. local inhabitants of the region. For the Initial names, the letter used for the team nickname is not the region name but the specific team name following the region name, for instance, "The O's" for Leyton Orient, "The R's" for Queens Park Rangers, and 'The U's" for Colchester United. That might suggest that the region name is taken for granted, and the distinctive part in the official team name is the non-regional name; therefore the football nickname source falls on this word. Taking that as the nickname of the team assumes the uniqueness of the name within the region, i.e. it can be regarded as the prominent aspect of the region and can typically represent that region. These football nicknames hence can make people feel they belong to the team since they belong to the region.

The club name related nicknames and the local particularity related nicknames achieve this effect through auditory impression: The shortened names, the derived names, the specific pronunciation, the initial names, and the names of local particularities all excite the fans and the people acoustically, evoking their sense of regional identity and thus winning strong popularity. 
Unlike the club name related nicknames and local particularity nicknames, which are sound-exciting, the home kit colour related nicknames are sight-exciting. What they exert is, for the most part, visual influence. This visual impression also implants in the fans and the people a sense of belonging. The visual stimulus can result in a specific conceptualization of the team and win visual support and popularity.

However, what the football nicknames of birds and animals evoke is mainly mental images through the visual effect of the coloured stripes of the home kit. They exert great influence upon the fans and people directly in the mind and win mental unification, integration and consolidation. What is more, although most of these nicknames (except "The Eagles" for Crystal Palace) are metonymically motivated as analysed above, it is inevitable for some of these nicknames to further metaphorically arouse in the fans' mind the knowledge of the birds and animals involved, i.e. the images of the animals and birds, especially those nicknames in which similarity is involved. That is, because of image schemas invoked by the colours or concepts, it might be possible that these bird and animal nicknames would result in metaphorical extension in the fans' mind. When they talk of or think of the names of the bird or the animal (as the football nicknames), it is feasible that they bring to their mind the most well-known advantages, characters and manners of the bird or the animal, and they might endow the football team with these features mentally ${ }^{70}$, or at least it is possible that they would have in mind related the image of the birds and animals to the teams. In other words, all the nicknames involving similarity to birds, animals, devils, plausibly evoke in the fans' minds the image, and/or the typical properties of these birds, animals and devils, though most derive from a metonymic process. That is the effect of mental impression; it empowers the football team to play on the field with all the fans on side physically and mentally.

As already argued earlier, football nicknames reflect specific conceptualization and categorization of the teams, which will influence others' view of the team and even of the team fans. Whether acoustically or visually or mentally, all the football nicknames were used to conceptualize and categorize the teams, and even the fans,

\footnotetext{
${ }^{70}$ This possibility applies only to those nicknames with similarity to the colour of animals. For other metonymically motivated nicknames, there is no such possibility, e.g. Australian National Rugby team is nicknamed as Wallaby, because Wallaby is metonymic for Australia (native to Australia); but it seems not possible to invoke any image or knowledge of Wallaby in the fans' mind.
} 
the region, and the area. Football nicknames may distinguish different categories of fans and achieve strong cohesive force and win wild popularity for the team. This function of football nicknames is the inherent cognitive role of metonymy motivating the nicknames and sometimes the function is further strengthened by metonymy-grounded metaphor.

\subsection{Summary}

Nicknaming is a language phenomenon as well as a social-cultural phenomenon, but it is also a cognitive phenomenon. Nicknames are studied in sports from the perspective of sociology and anthropology. Nicknames of sports teams, e.g. football teams, are not a specific person but functional collective of players. They are different entities in some aspect. Football team nicknames mainly focus on the home kit colour, the local particularity.

Nicknames as both a language and a cognitive phenomenon are largely motivated by metonymy. Football nicknames can be classified into some patterns such as Football Club Name Related, Home Kit Colour Related, and Local Particularity Related. Under these patterns lie some cognitive processes. In this case study, the analysis shows that the overwhelming cognitive process motivating these patterns is metonymy, including two variant forms of it, i.e. double metonymy and chained metonymy. The findings indicate the chained metonymy is more frequently employed in the cognitive process. In the data analysis, two other cognitive processes are also found, one is the combination of metonymy and metaphor, the other is metaphor. But the analysis also implies that metonymy is requisite for metaphor to take place; this suggests that metonymy is a crucial cognitive mechanism in football nicknaming.

Since football nicknames are strongly motivated, they bear some functions. The analysis indicates that these functions may be operated through auditory, visual and mental excitation usually under the motivation of metonymy.

Nevertheless, this is only a case study, and the data base is comparatively narrow. More data analysis is needed to see how far metonymy as a cognitive mechanism operates in football team nicknames. The case study is confined to the top levels of 
English football clubs, there is vast space left unexplored; for example, what about those football nicknames in other countries such as Spain, Brazil, Argentina? Is there possibly any difference in football nicknames between different areas and countries? It is plausible to have the same nickname with different football teams, in this case, is there any difference in terms of cognitive process and function? In addition, in the case study, there are some football club teams who share the same nicknames. Are there any other conceptual differences involved besides the underlying cognitive mechanism? These questions are worth future effort to have a comprehensive understanding of football nicknames from the perspective of Cognitive Linguistics as well as the perspective of sociolinguistics and psycholinguistics. 


\section{PART THREE METONYMY IN COMMUNICATION}




\section{Chapter 8 Metonymy in Dialogic Discourse: Structuring and Developing}

Metonymy in Cognitive Linguistics is typically viewed as an intra-domain mapping in which the source provides mental access to the target, with PART-WHOLE as the prototypical relation between the source and the target. In PART ONE, it was argued that metonymy is an innate cognitive mechanism involving two successive processes: PART-WHOLE FORMING and PART-WHOLE/PART RELATING, with the former guiding or potentiating the latter. What current Cognitive Linguistic study of metonymy looks at is exclusively the latter process, in some sense, neglecting the indispensible former one. However, it has been illustrated that the PART-WHOLE FORMING process is more crucial in the mind and this was further evidenced in language in PART TWO, e.g. in grammaticalization, in meaning prototype development, and in motivating daily language use such as football nicknames, in which the function and contribution of metonymy as a natural cognitive mechanism starts to unfold. This part will examine how metonymy contributes to the structure and development of dialogues in immediate interactive communication, and contributes to the interaction as a whole discourse in terms of meaning-making, decision-making, and problem-solving; how its two processes interact and function in communication. Communication is used in this research specifically as face-toface interaction, especially in the form of dialogue, or rather in dialogic discourse. This chapter focuses on metonymy and dialogic discourse from the conceptual perspective, examining how metonymy structures and develops dialogic discourse; and the following chapter will focus on how metonymy contributes to meaningmaking, decision-making and problem-solving in dialogue.

\subsection{Metonymy and dialogic discourse}

Since the cognitive turn of research on metonymy, a large number of studies have been done, in terms of research area, on the distinction and interaction between metonymy and metaphor (e.g. Barcelona, 2000b; Dirven \& Pörings, 2002; Warren, 1995), the nature of metonymy as a cognitive process or model (e.g. Goossens, 1995; Lakoff, 1987b; Panther \& Radden, 1999), metonymy as a cognitive motivation of language structure and language use (e.g. Barcelona, 2004; Barcelona, 2005a, 2007a; 
Brdar, 2007; Langacker, 2004; Panther \& Thornburg, 2003b; Panther et al., 2009; Radden, 2007b; Ruiz de Mendoza \& Otal Campo, 2002); in terms of approach, on metonymy from the diachronic perspective (e.g. Allan, 2008), from the corpus-based perspective (e.g. Stefanowitsch \& Gries, 2006), from the semiotic perspective (e.g. AI-Sharafi, 2004), from the discourse perspective (e.g. Campo, 2005).

Metonymy in discourse has also been examined within the Cognitive Linguistic framework, e.g. the meaning-construction role of metonymy in discourse and pragmatic inference (Barcelona, 2005b, 2007a, 2007b; Panther, 2005, 2006; Panther \& Thornburg, 2003b; Radden, 2007b). However, it seems that this examination tends to focus on specific metonymically-motivated names, words or sentences in the discourse, e.g. Musson \& Tietze (2004), Cornelissen (2008), and Riad \& Vaara (2011). And furthermore, the discourse in which metonymy is looked at is mainly narrative, in the form of paragraphs, sentences, e.g. Barcelona (2005b, 2007b), Pankhurst (1999). Sometimes the discourse employed is interactive, e.g. a conversation or dialogue such as the following (for more examples, see Barcelona, 2003a, 2005a):

\begin{tabular}{|l|l|}
\hline A: & How did you get to the airport? \\
\hline B: & I waved down a taxi. \\
\hline & \\
\hline
\end{tabular}

But they appear to be isolated one-turn talks. Or even if the talk is more than one turn, the focus is liable to be on a specific component of the utterance such as company names, building or office names (Cornelissen, 2008; Musson \& Tietze, 2004; Tietze, 1998).

Another study of metonymy in interaction is speech act metonymies. The study of speech act metonymies (e.g. Panther \& Thornburg, 1998; Thornburg \& Panther, 1997) provides good examples of how metonymy operates in immediate interaction in terms of speech acts. For instance:

\begin{tabular}{|l|l|}
\hline A: & $\begin{array}{l}\text { Osmond, I want my tape! ... No thank you, you haven't got one big } \\
\text { enough. Osmond, [shouting] I want my tape! ... You fucking cunt. } \\
\text { Claire [tell him] }\end{array}$ \\
\hline
\end{tabular}




\begin{tabular}{|l|l|}
\hline B: & [unclear $]$ \\
\hline A: & Claire, tell him I want my tape. \\
\hline B: & She wants her tape ... \\
\hline C: & [unclear $]$ \\
\hline A: & Osmond, you're out of order you know. \\
\hline B: & [unclear $]$ \\
\hline C: & [unclear $]$ \\
\hline A: & I apo= \\
\hline C: & {$[$ unclear $]$} \\
\hline A: & $\begin{array}{l}\text { I apo= What did you say? Did you say fuck Saira? ... Oh, Osmond, } \\
\text { come on, give me the tape don't be out of order. }\end{array}$ \\
\hline C: & Shut the fuck, woman! \\
\hline &
\end{tabular}

In this example, "I want ..." statement is not really a statement, but metonymically a request. This well illustrates speech act metonymies in dialogue, in which a direct speech act is metonymically transferred to an indirect speech act such as NECESSITY FOR MOTIVATION in "I must have a talk with you" (I MUST HAVE A TALK WITH YOU FOR I WANT TO HAVE A TALK WITH YOU), ABILITY FOR MOTIVATION in “... I can give you a lift” (I CAN GIVE YOU A LIFT FOR I WANT TO GIVE YOU A LIFT). These illocutionary metonymies are supposed to take place in Action Scenarios, which are comprised of four components, i.e. the BEFORE (preconditions which enable a physical action, legitimize a social action or motivate an action), the CORE (the essential features of the action itself), the RESULT (the immediate outcome of a successful performance of the action), and the AFTER (intended or unintended consequences of the action which are not its immediate result) (Panther \& Thornburg, 1998, pp. 758-759; Thornburg \& Panther, 1997, p. 207). All these components can trigger the whole action scenario, relating the PART to the WHOLE or the PART to another PART within the same scenario. Panther \& Thornburg (1998, p. 761, p. 768) propose two hypotheses regarding these PART-WHOLE relating, reproduced here as follows:

\section{Hypothesis 1}


The more distant a speech act scenario component is from the CORE, the weaker is its ability to evoke the scenario metonymically. In other words, the more conceptually removed a component is from the CORE, the less likely that component will be in a stand for (metonymic) relation to the scenario as a whole.

\section{Hypothesis 2}

The more components of a scenario present in a discourse, the easier it is to identify the scenario and the more likely even a relatively peripheral component can stand metonymically for the scenario.

These hypotheses suggest that each component can metonymically provide access to the WHOLE action scenario, granting the contextual situation allows. For instance, in the following conversation the existential proposition "There's the Jungle Book" functions metonymically as a directive (suggestion/recommendation), though it is more distant from the CORE component of a directive action:

\begin{tabular}{|l|l|}
\hline A: & $\begin{array}{l}\text { No, cos the thing is, my dad wants to go and see, a film sometime } \\
\text { near my birthday. }\end{array}$ \\
\hline B: & {$[$ laughs] } \\
\hline A: & So, erm, which I don't mind cos \\
\hline B: & What film? \\
\hline A: & Dunno. But I don't wanna see a film, that, my parents are gonna \\
\hline B: & watch with me. I wanna watch [unclear] \\
\hline A: & See I wanna see Accidental Hero. \\
\hline B: & Please ! \\
\hline A: & That looks good. \\
\hline B: & I know, but I mean, come on! \\
\hline A: & Well what else is on actually? \\
\hline B: & There's the Jungle Book. \\
\hline A: & Yes! \\
\hline B: & We could take Warner. \\
\hline
\end{tabular}




\begin{tabular}{|l|l|}
\hline A: & No. \\
\hline \multicolumn{2}{|c|}{ (Adapted from Panther \& Thornburg, 1998, p. 767) } \\
\hline
\end{tabular}

Although speech act metonymies are significant in dialogic understanding, what counts is not merely the speech act itself, but rather the general act scenario, or rather Event Scenario, i.e. the metonymically-formed experience pattern; speech act is only a special part of it and becomes possible only after the pattern is formed through PART-WHOLE FORMING. Consider an example:

\begin{tabular}{|l|l|}
\hline A: & $\begin{array}{l}\text { Where do you live? I'm gon na take you home. You're gon na take your } \\
\text { baby. Screw your car if they tow it away, because you're gon na end up } \\
\text { jail, and they're gon na take your baby away }\end{array}$ \\
\hline B: & Don't, please don't freak her out. \\
\hline C: & No, I'm not freaked out. \\
\hline B: & $\begin{array}{l}\text { There's nothing to say that you're going to jail. But what I do know } \\
\text { right now... }\end{array}$ \\
\hline A: & She's drinking, and she's got behind the wheel. It's reality. \\
\hline B: & $\begin{array}{l}\text { Why am I gon na, she gave me her keys. She voluntarily gave me her } \\
\text { keys. She's making responsible steps. }\end{array}$ \\
\hline C: & Okay, you know what... \\
\hline C: & I'll just take my baby to the doctor. \\
\hline C: & I'm just taking my baby to the doctor. \\
\hline B: & Do not let her pick up the baby. Please... \\
\hline (Adapted from COCA ${ }^{71}:$ ABC-Primetime: What would you do? May 21, 2010) \\
\hline
\end{tabular}

In this example, the utterance containing "behind the wheel" might have a speech act metonymy, e.g. a statement for a protest, but it has nothing to do with "behind the wheel". Nevertheless, "behind the wheel" is part of DRIVE action scenario, and it is the BEFORE component, in Panther \& Thornburg's term. In order to drive, you first have to get behind the wheel, start the engine, check mirrors, fasten the seat belt, and

\footnotetext{
${ }^{71} \mathrm{COCA}$ is short for the Corpus of Contemporary American English, which is the the largest freelyavailable corpus of English created by Mark Davies of Bragham Young University in 2008. The corpus contains more than 425 million words of text and is equally divided among spoken, fiction, popular magazines, newspapers, and academic texts and is updated once or twice a year (the most recent texts are from March 2011). For detail see http://corpus.byu.edu/coca/
} 
control the gear, etc. But in this case, speech act metonymies cannot alone account for this situation.

Apart from this, there is another situation which may not be interpreted with speech act metonymies, and not even with the general Action Scenario. The following example shows another speech act metonymy in conversation, i.e. a question is supposed to be metonymically transformed into a request with intention to the boy, which is unfortunately misunderstood:

A boy on the bus had a running nose, but he did nothing about it. So a young lady, who was sitting beside him and could not bear it anymore, posed a question.

\begin{tabular}{|l|l|}
\hline Lady: & Do you have a handkerchief? \\
\hline Boy: & I would not lend it to you even if I had one! \\
\hline \multicolumn{2}{|c|}{$\quad$ (Translated from Fu, 2005) } \\
\hline
\end{tabular}

The lady presented a request by way of asking a question, implying that the boy needed to clean his nose. From the answer, it can be said that the boy did make sense of the lady's question-for-request metonymy, in that he inferred that the lady asked such a question because she needed (required) a handkerchief from him, i.e. a question-for-request metonymy; but he misunderstood her intention to require him to clean his own nose. It is not likely that the boy misunderstood the speech act metonymy. What he misunderstood was the intention of the lady, i.e. the goal in Embodied Cognition terms. This also suggests that speech act metonymies, though they function in dialogues or conversations, might not be as conceptually crucial in communication as supposed; and these illocutionary metonymies need to be interpreted by the cognitive approach to dialogic discourse in order to have their role and place in metonymy research from the conceptual perspective. In brief, it seems that speech act metonymies cannot cover the whole picture of an interactive dialogue. Even if they can have a structuring function, this function, for the most part, only serves the local talk turns. In terms of the structure and development of a dialogic discourse, this role appears to be weak. 
However, metonymy in dialogic discourse beyond the speech act has not been fully or systematically addressed by the Cognitive Linguistics literature, though it may be touched upon, e.g. metonymy as guiding implicature across longer texts (Barcelona, 2007b). In addition, the Cognitive Linguistic scholarship claims a usage-based account for meaning, language, and language change and variation. And all these are inherently dialogic (Bakhtin, 1981). Therefore, examining metonymy in actually occurring dialogic discourse can lead to a clear idea of how metonymy conceptually contributes to the dialogue, e.g. how it structures and develops the dialogic discourse, how it contributes to meaning making, decision making and problem solving.

In Bakhtin's (1981) view, utterance (e.g. words, language, and literary works) is dialogic in nature, and even inner speech is dialogic; they are dialogic in that every utterance is a response to relevant previous utterances and simultaneously generates or anticipates a response in the receiver. Although this broad view of language as dialogue and of meaning as interactive product is acceptable, dialogic discourse in this research is confined to discourse in the form of dialogue, i.e. dialogues between at least two actual persons and with at least one talk turn for each person, e.g. conversation, meeting discussion, email exchange (Wallace, 1999, 2000a, 2000b), online group discussion (Wallace, 2001), radio interview, etc.

\subsection{Metonymy and logical thinking}

As an innate cognitive mechanism involving PART-WHOLE FORMING and PART-WHOLE RELATING, metonymy seems to be the essential feature of Embodied Cognition and underpins human cognitive reasoning, which is embedded in the PART-WHOLE FORMING and PART-WHOLE/PART RELATING processes.

There are in general three types of reasoning: Induction, deduction, and abduction, which involve three propositions each, i.e. the Law, the Case, and the Result (the so-called syllogism). Deductive reasoning applies a law to a case and predicts a result, for example: All men are mortal, Socrates is a man, and therefore Socrates is mortal. Inductive reasoning proceeds from observed cases and results to establish a law, e.g. Socrates is a man, Socrates is mortal; Abraham Lincoln is a man, Abraham Lincoln is mortal; therefore all men are mortal. Abductive reasoning proceeds from 
an observed result, invokes a law and infers that something might be the case, e.g. given the fact that Socrates is dead, it is related to the general law that all men are mortal, and the inference can be made that Socrates was a man (Anderson, 1973, pp. 774-776; Hopper \& Traugott, 1993, pp. 39-40; Walkden, 2011, pp. 230-231).

Some points are implied in them. One is that all these general reasoning types take the form of a series of propositions. But as it is well known, propositions are statements comprising concepts. That is to say, these propositions are all products of categorization and conceptualization. PART ONE of this thesis has tentatively argued that metonymy seems to prominently operate in categorization, conceptualization, memory and generalization. In infancy, perceptual and conceptual categories are constructed out of specific (sensorimotor) experience, and this process is metonymy of SPECIFIC TO GENERAL/GLOBAL. And this kind of general/global category will be enriched, specified, and updated, with each increment of experience. Reasoning is inclined to be possible and easier within the general/global patterned experience. This is the second point, which suggests that these general types of reasoning are, for the most part, viable due to the Law that is already established, except the enumerative inductive reasoning, which is more like the PART-WHOLE FORMING process and constructs a general Law out of specific cases). Taken in the aggregate, it seems that metonymy not only operates in proposition construction, but also underpins these general types of reasoning between propositions. It is likely that metonymy underlies logical thinking. Once the embodied experience is patterned (i.e. the whole is formed and formatted), a stereotype would be set up and thus guide reasoning and inference. In fact stereotyping might be essentially motivated by metonymy. ${ }^{72}$

In dialogic discourse, e.g. in interviews, metonymy may be found to motivate the dialogue in terms of structure and development, such as from specific cases to a general view (e.g. evaluation, evaluative conclusion), or from an overview or a formed general framework to a specific case or situation. It might be said that interviews in general, and broadcast interviews in particular, are ideally guided by

\footnotetext{
72 This is only a logical inference, based on the cognitive analysis of language in the literature. But the empirical test really needs psychological or neuroscience experimentation.
} 
the interviewer, but this leading has to do with what the interviewee is talking about or has said, or the main topic of the interview, otherwise it will make the interview incoherent or illogical. This might be where metonymy functions in terms of structure and development. The following sections will be devoted to the analysis of metonymy as structuring and developing interviews.

\subsection{Discourse data}

The dialogic discourse used here for metonymic analysis primarily comes from the spoken part of ICE-NZ (The New Zealand Component of the International Corpus of English), and WSC (The Wellington Corpus of Spoken New Zealand English), complemented by personal collection from movies and newspapers.

ICE-NZ was completed in 1999 as part of ICE (The International Corpus of English ${ }^{73}$ by the School of Linguistics and Applied Language Studies, Victoria University of Wellington. ICE-NZ consists of 600,000 words of speech in the form of private and public dialogues, collected between 1990 and 1994. Private dialogues are comprised of direct conversations and distance conversations, and public dialogues involve class lessons, broadcast discussions, broadcast interviews, parliamentary debates, legal cross-examination, and business transactions (Vine, 1999).

The WSC was also developed by the School of Linguistics and Applied Language Studies, Victoria University of Wellington and completed in 1998. It is comprised of one million words of spoken New Zealand English collected in the years 1988 to 1994, with ninety nine percent of the data collected in the years 1990 to 1994 and the rest collected in 1988 and 1989. The corpus consists of 2,000 word extracts (where possible) and comprises different proportions of Formal Speech/Monologue (12\%), Semi-formal Speech/Elicited Monologue (13\%) formal, Informal Speech/Dialogue (75\%), with over 900,000 words in dialogues such as Conversation, Telephone

\footnotetext{
73 The International Corpus of English (ICE) began in 1990 with the primary aim of collecting material for comparative studies of English worldwide. Twenty-three research teams around the world are preparing electronic corpora of their own national or regional variety of English. Each ICE corpus consists of one million words of spoken and written English produced after 1989. For most participating countries, the ICE project is stimulating the first systematic investigation of the national variety. To ensure compatibility among the component corpora, each team is following a common corpus design, as well as a common scheme for grammatical annotation. For details see http://icecorpora.net/ice/index.htm
} 
Conversation, Oral history Interview, Social Dialect Interview, Radio Talkback Broadcast Interview, Parliamentary Debate, and Transactions and Meetings (Holmes, Vine, \& Johnson, 1998). There is also some overlapping between ICE-NZ and WSC in the categories, for example, Telephone Conversation, Business Transactions, and Parliamentary Debate, while the dialogic discourse in WSC is much larger than that in ICE-NZ.

These corpora were not established specifically for discourse or dialogue/conversation analysis but for the analysis of the national or regional features of New Zealand English (or other regional variety of English). In the dialogue part most of the conversations, interviews, and meetings are not complete dialogues/conversations, but fragments. The preliminary analysis finds that broadcast interviews are comparatively complete and are enough for analysis in terms of number, e.g. WSC contains 40 broadcast interviews, and ICE-NZ has 30 broadcast interviews and discussions, therefore broadcast interviews are specifically focused in the examination in both of ICE-NZ and WSC, while attention is also paid to conversations, and business transactions and meetings.

\subsection{Metonymy in interviews}

In PART ONE it was argued that metonymy operates in and characterizes conceptualization, categorization, memory, generalization and making inferences since infancy. It was further contended that metonymy is an innate cognitive mechanism involving PART-WHOLE FORMING and PART-WHOLE RELATING processes, with the FORMING process preconditioning the RELATING process, as reiterated at the outset of this chapter. These two processes interact in dialogic discourse without conscious awareness and the interactions between them help structure and develop the dialogic discourse. To illustrate this point, now let's see some examples from ICE-NZ ${ }^{74}$ and $\mathrm{WSC}^{75}$.

\footnotetext{
${ }^{74}$ Another point needs to be made clear, most of the interviews in ICE-NZ are not whole complete ones, I just choose those interviews which are comparatively complete and may be better fit for my analysis.

${ }^{75}$ There are a number of interviews in these two corpora. However, it is not possible to analyse all or most of them in this limited space. What is managed here is to give detailed analysis of typical interviews.
} 
The first example is from ICE-NZ: S1B-41. It is an interview between a male documentary interviewer (I) and a former female teacher (F) about F's childhood experience with her mother as a driver. This interview in the corpus consists of 9 talk turns by $\mathrm{F}$ and 8 talk turns by I, and F talks much more than I in each turn. What is followed is the main point of each talk turn in the interview ${ }^{76}$, and then the analysis in terms of metonymy.

In her first talk turn (this is not the very beginning of the interview but the first talk recorded in the corpus), $\mathrm{F}$ mentioned that their parents bought a Model $\mathrm{T}$ Ford car and recalled their funny and risky experience of driving the car up the slips, over chasms, and across the creek between the gorge to her mother's family home at Ormond. Following this and regarding the place, I puts forward the larger place Gisborne. This connection of talk turns between F and I relies on the relationship between Ormond and Gisborne. Ormond is part of Gisborne area in terms of administrative division. The mention of Ormond by $\mathrm{F}$ activated I's knowledge framework of the administrative location of Ormond in Gisborne. This might be termed SMALLER PLACE FOR LARGER PLACE IT BELONGS TO IN ADMINISTRATION, a subtype of PART FOR WHOLE metonymy. It therefore seems to be the PART-WHOLE RELATING process involved here in developing the interview.

Meanwhile, I's utterance "this was out er on the Gisborne side" is possibly a speech act metonymy as well in the interview. It could be that I's assertion simultaneously proposes an interrogation (like a rhetorical question), that is, his statement attracts a positive answer from F. Thus F's following utterance "on the Gisborne side yes" sounds very natural in the dialogue development. This structuring function is likely to be served by the ASSERTION FOR QUESTION speech act metonymy.

Then F goes on to talk further about their experience with her mother: The occurring time (in the spring), what they did while staying with their grandparents, grandmother taking care of her father while they were away. It seems that $\mathrm{F}$ is wandering away from the topic in her utterance. To bring her back to the topic, I then

\footnotetext{
${ }^{76}$ For details, please refer to the Corpus ICE-NZ: S1B-41. The dialogues from ICE-NZ and WSC are over 2000 words each; the full dialogues under discussion in this research are not reproduced here due to limited space in the thesis.
} 
breaks in and refers back to the Model T Ford car mentioned earlier in F's first talk turn, uttering "now the Model T Ford, it was quite a versatile kind of vehicle". I cleverly picks up the vehicle of her mother's driving experience and brings it to the fore for further talk of her mother's experience. By mentioning part of what $\mathrm{F}$ has talked about earlier, I activates F's knowledge of the car and the risky experience with her mother in the car and brings her thought back to the vehicle and that experience. This talk turn also shows that the interviewer guides the interviewee in the interview, and this is the general situation in interviews. However, this guide seems to relate to what has been covered in the interview, i.e. bringing part of what has been talked about or mentioned earlier in the dialogue (which might be in the inferior status when first mentioned) to the focus status for further talk. The process underlying this guide is GROUND-FIGURE, that is, a WHOLE-PART RELATING process. This function can be said to be served by "now the Model T Ford". Thus, "Yes, it it is certainly was" is naturally F's following utterance in her turn. In the meanwhile, just like I's first talk turn "this was out er on the Gisborne side" analysed above, I's utterance "it was quite a versatile kind of vehicle" proposes an ASSERTION FOR QUESTION speech act metonymy due to the situation of interview. Another point is, "it was quite a versatile kind of vehicle" by I comes from his generalization from the hard driving experience told by $\mathrm{F}$. This may be termed as PROPERTY FOR EVENTS; the underlying inference is that it must be a versatile one since the car could go well in those hard conditions. It is very likely that the process underlying the structuring connection between F's and I's utterances here is from EVENTS TO PROPERTY, i.e. those challenging cases embodies the versatile quality of the car; it is from the specific cases to the general/overall quality. It might be termed as SPECIFIC TO GENERAL process.

F then goes on to talk about the versatility of the car in their experience of river crossings, mentioning chassis, magneto, and drying out. When $\mathrm{F}$ goes away to talk about other stuff and seems to trail off into silence, I immediately picks up the "river crossings", which is part of what $\mathrm{F}$ has just mentioned in her talk of the car. This topic, on the one hand, is related to driving experience; on the other hand, is also a general topic for further specification in the talk, which can be evidenced in F's following turn. If "chassis" or "magneto" is selected for further talk, then first, it would be too specific to keep the talk going; and second, it would draw the talk far 
from the driving experience or memories. This is a very wise choice, thus keeping everything in the talk to the main topic of the interview and keeping it proceeding smoothly. It once again shows the interviewer's guidance in the interview, which is often done by picking up some specific part of what has been covered in the talk as the topic, that is, through GENERAL-SPECIFIC or GROUND-FIGURE process, a subtype of PART-WHOLE RELATING. This time, not like what I has done in the first two turns which each proposes an ASSERTION FOR QUESTION speech act metonymy, I's utterance "you'd remember these river crossings as children, wouldn't you?" does not involve a speech act metonymy. It is directly a rhetoric question, inviting a positive confirmation from $\mathrm{F}$, which helps develop the dialogue. Nevertheless, the general topic "these river crossings" also poses a specific development of the topic. That is why $\mathrm{F}$ continues to talk about the river crossing experience after she replies "yes I yes I do". In this sense, the GENERAL TO SPECIFIC process helps with the further development of the dialogue.

When $\mathrm{F}$ talks further about their river crossing experience, giving some specific examples of the dreadful situation and her mother's resourcefulness and can-do attitude, I accordingly generalizes and indicates that they were never put into a hopeless situation because of the mother, by saying "so you were never stuck there hopelessly, you know, where the car wouldn't drive". This might be termed as GENERAL FOR SPECIFIC CASES, i.e. the process underlying the structuring connection between F's and I's utterances is from the specific to the general, in which the specific activates the general. Specifically it can also be said to be ACTION/EVENT FOR ABILITY/CAPABILITY and ACTION/EVENT FOR RESULT. Her resourceful action in the frightening situation embodies her determination and intelligence as well as brings them hope and cheer, thus they were never hopeless even in the terrible situation. At the same time, this utterance by I also proposes an ASSERTION FOR QUESTION speech act metonymy, as analysed in I's first two talk turns. Thus F follows I's utterance by saying "not that I remember" and then starts to give her general opinion of her mother "she must have had a guardian angel I think" and the reason and specific cases.

It is plausible that I's generalization encourages $F$ to talk about another awful experience and how her mother determinedly dealt with the situation. I subsequently 
makes an evaluative generalization from those specific cases that her mother must have earned considerable respect from the people living and working around there. By the same token as I's talk turns 2 and 4, here in his fifth turn, I gives such a conclusion, which comes from the cases F just mentioned in her fifth turn. This is obviously from the specific to the general and may be termed EVENTS FOR RESULT as well. In I's view, F's mother acted so determinedly in those situations, even more than men, that she would have earned considerable respect from those living and working there. The underlying process connecting F's and I's utterances is probably from EVENTS/CASES TO RESULT. This, together with I's turns 2 and 4, are all situations of PART-WHOLE FORMING: From the specific cases to form a general conclusion or opinion, which is like how metonymy operates in categorization, as argued in Chapter 3. That is, I is categorizing her mother as a certain group or type of person based on specific cases. Simultaneously, the utterance (I's fifth turn) "she would have been a woman er who would have earned considerable respect from the people living in the gorge and also from the workmen who came to build the road" poses an ASSERTION FOR QUESTION metonymy, in which the conclusive statement invites a positive reply from $\mathrm{F}$. That is why $\mathrm{F}$ subsequently says "yes yes she did er", and goes on with her mother's kindheartedness and help that earned her the greatest respect from those people.

One of her mother's kind-heartedness is hospitality. She made her house a stopover for those people who could not go any further due to unlucky incidents such as that they got stuck over the hill, the engine boiled, the car conked out, and they were stopped by a slip in the gorge. I might have got some knowledge of their experience before the interview, because his sixth turn "your house was something of a stopover, wasn't it" follows F's "I think she felt very sorry for them". In her sixth turn, F's utterance "she was known as a very kind-hearted person and would always help in whatever way she could" may activate I's knowledge of their house as a stopover for those people at that time. This utterance by $\mathrm{F}$ is her categorization of her mother's personality, and making her house as a stopover for them is a specific case of this personality. This process could be termed as PERSONALITY TO CASES, i.e. making her house as a stopover for those people in need embodies her kindheartedness; or in other words, it is the process of GENERAL TO SPECIFIC that coheres I's and F's utterances here. On the one hand, I's sixth talk-turn is a rhetoric 
question, and it attracts a positive answer here. On the other hand, "your house was something of a stopover" invites further talk of specific cases. Therefore, F first gives a positive reply and then tells some specific cases of her house as a stopover in her seventh talk-turn.

After F talks about how they treated those visitors in her seventh turn, I says that her family is in fact still remembered. This connection involves a metonymic inference: Those people were so grateful to them for their kindness and hospitality that they named the place after their family (Traffords Hill) (CAUSE TO EFFECT/RESULT, with the cause as the source); and the place where they lived is now still called Traffords Hill, they must be still remembered (EFFECT/RESULT TO CAUSE, with the result as the source). This inference involves a metonymic chain: It goes first from the reason to the result, and then goes from the result to the reason. And F's following talk proves I's inference. In the three factors of the syllogism, the Case/Cause/Reason (they treated people well), the Result (people name the place after their family, i.e. Traffords Hill), the Law (people are remembered by naming the place where they live after them), the Law is actually already established encyclopaedic knowledge; when the Case and the Result are co-present, with the Law established, they form a corollary, resulting in I's utterance "in fact your family's still remembered in Waioeka Gorge area there with Traffords Hill." This metonymic inference lies in event scenarios. As mentioned in Chapter 6, a typical event scenario involves people, action/movement, time, place, objects, etc. Once a patterned experience is achieved, e.g. a stereotype, any one of those scenario elements can be metonymically triggered or accessed, and can provide access to the whole patterned experience, in the appropriate situation.

But the information of naming the place after F's family is only I's knowledge that underpins his seventh talk-turn, it is not actually stated and thus not known to the audience. This statement is more likely to be an ASSERTION FOR QUESTION metonymy and needs a confirmation. To enforce this point, I immediately says "it's still called Trafford Hill, isn't it?" This utterance is a rhetoric question, and it also poses a FACT/EFFECT FOR CAUSE metonymy in addition to inviting a positive reply. That is, I's statement of the fact that the place is nowadays still called Trafford Hill would make F tell of the reason in her next talk-turn. So does F subsequently. 
This process connecting their utterances is therefore a combination of CAUSE TO EFFECT and EFFECT TO CAUSE.

The above analysis shows that in this interview, metonymy underlies the development of the dialogue and helps structure it into a coherent discourse. It helps construct the interview in a variety of forms.

Here is another example from WSC (WSC\#DGI158 ${ }^{77}$ ), which is an interview between IH (male) and AW (female) about a new law --- the Consumer Guarantees Act.

The structuring function of metonymy is clearly evidenced in this example, at least between the talk-turns. The previous interview involved both PART-WHOLE FORMING and PART-WHOLE RELATING (the various subtypes of each process). The present interview predominantly comprises PART-WHOLE RELATING (i.e. from the general to the specific), sometimes the PART-WHOLE RELATING comes from IH's own information about the Act (from his overall knowledge of the Act to some specific aspects of it), and sometimes it develops from AW's reply (IH's talkturn topic comes from AW's informative reply to IH's earlier question). The following is the analysis of it in terms of dialogic discourse structure and development.

IH's first talk-turn poses an ASSERTION FOR QUESTION metonymy, which invites a positive reply, so does AW immediately in her turn. IH's first utterance is in fact a topic introduction of the Consumer Guarantees Act, together with AW's involvement in the Act. But this statement needs AW's confirmation. In her reply, AW gives some specific information about the Act besides her confirmation, such as what it is, when it came into force.

In the second talk-turn, IH presents another aspect of the Act: Its Maori name. This information is probably from IH's knowledge of the Act before the interview. In AW's first turn, she has already mentioned the nature of the Act, the time the Act

\footnotetext{
${ }^{77}$ WSC expresses this dialogue is from the corpus WSC, \# is the phrase marker, DGI158 is the extract code. In the following such as $1: \mathrm{IH}$, the number is the phrase number and $\mathrm{IH}$ is the speaker identification. In the corpus the phrase/unit number is originally calculated by 5 , but here it will be serially numbered, starting with 1 , with those unnecessary transcription symbols deleted, as did to ICE-NZ.
} 
came into force. Now is the Maori name of the Act. Like IH's first turn, this utterance is also an ASSERTION FOR QUESTION metonymy, in which the statement "I also understand that this project you're involved in has a Maori name" invites a positive reply from AW. This naturally brings in AW's utterance "That's right”. But this assertion is presented to AW not only for her confirmation but also for some further information about the reason for giving the Act a Maori name. Hence, it also involves a FACT FOR REASON metonymy, i.e. a variant form of EFFECT FOR CAUSE metonymy, with the fact as the source.

IH's third turn still follows his own thought of the Act after the general introduction of it, i.e. his knowledge of the Act as a joint project. But this time, IH is different from his first two talk-turns. In the first turn, he uses a statement followed by a direct request. In the second turn, he only uses a statement. These two turns are both ASSERTION FOR QUESTION metonymies. In the third turn, IH uses a statement followed by a direct question. The Act as a joint project is also an aspect of IH's knowledge of the Act, or in one word, aspect of the Act. If the Act is the general topic of the interview, these specific aspects are all component knowledge of the Act. The Act and these aspects could be said to be in GENERAL-SPECIFIC relation. It is feasible that the general introduction of the Act activates some related specific aspects such as the above.

By saying "understand" three times, IH is using his own formed knowledge about this law to guide the interview. Following his own knowledge about the Act, IH then asks further specific information about the joint project: "With whom?" This time, it is not a mere statement for request, but a direct question for specific information. Following AW's answer and information, which speaks of consumer affairs, IH starts to focus on more specific information about the Act itself. The first three turns are all about the background or general information of the Act. This is true with event scenarios. An event scenario involves the event itself and background information such as when, why, how, where, what. The Maori name, the time the Act came into force, the fact that it is a joint project, can be said to be peripheral information of the Act; what the Act is about, then, is the core of the Act, which provides the basis for the questions that follow. 
Both the name of the Consumer Guarantees Act and AW's reply to the question about it being a joint project contain the word "consumer"; it is natural that IH starts to ask what a consumer is (the fourth turn). Therefore the movement of the dialogue is overall from the general to the specific, from the peripheral to the core. Following AW's answer to what a consumer is: "Anybody who buys or uses goods or services", IH immediately poses another question (the fifth turn) "what is a good?"

After that, IH mentions (the sixth turn) "now this act talks about guarantees". This comes from the name of the Consumer Guarantees Act, i.e. from the general knowledge of the Act, thus the process of GENERAL TO SPECIFIC is involved here. Nevertheless, it may still follow naturally from AW's reply about what goods are. Goods are generally guaranteed, but what is a guarantee? Therefore here is a good position to pose the question about guarantees. Both questions arise out of a movement from the whole to the part. AW's reply: "A guarantee quite simply is a promise" brings in another question from IH (the seventh turn) "so why do we need a new act?" For IH, promises are common and have to be kept, and since there is already a Goods Act, why do we need to have a new act? In event scenarios, the reasons (why) an event happens of course are part of the event; after the event occurs (the Act passed in this case), sourcing the reason is surely a process of WHOLE TO PART.

The next question (the eighth turn) "so what are the main points of the act" follows AW's reply that the new Act brings services as well as goods into a regime of guarantees. From the viewpoint of event scenario, this question is the core part. From the viewpoint of structure, this question naturally follows. The idea that the Act guarantees both goods and services may sound rather general. It is natural to ask about the main points of the Act. Therefore the underlying process here is also from the general to the specific.

IH guides the interview to more specific information about the core part of the Act by uttering (the ninth turn) "when you talk about guarantees about goods, what are these guarantees?" The pattern of the dialogic discourse now comes to more specific questions: Guarantees of goods and services $\rightarrow$ guarantees of goods. In AW's reply to the question of guarantees, AW mentions "acceptable quality", "fit for any particular purpose", "must match any description or sample", "reasonable price", 
"seller having the right to sell", "available repair and spare parts", and "manufacturers' guarantee". All these become topics of IH's following questions (the tenth turn to the sixteenth turn). For example, From AW's reply, IH picks up "acceptable quality" to further his question "now what do we mean by goods of acceptable quality?" Relating to "being fit for a particular purpose", IH poses a question, actually an illocutionary metonymy again (ASSERTION FOR QUESTION), i.e. "now we talk about them being fit for a particular purpose" for "What do we mean by being fit for a particular purpose" or "Can you give some explanation". Relating to "must match any description or sample", IH poses a question after a statement, just as he did earlier, "what do we mean by that?" Among IH's later questions, "what about price" is related to "reasonable price"; "what about repairs and spare parts" is related to "repair facilities and spare parts"; "what about the right to sell" is related to "that the seller has the right to sell the goods"; "what about the manufacturer's guarantee or warranty" is relating to "manufacturers' guarantees".

Following the manufacturer's guarantee or warranty question, or rather, after all these questions of various guarantees, it is highly likely to pose a question like "what happens when the guarantees are broken" (the seventeenth turn). This is the special situation. The Act must cover both the regular and the special cases. Thus this question also fits into the general- to- specific rubric. After AW answers about faults that are not serious, IH immediately asks about serious faults (the eighteenth turn) "what happens if there are serious faults", which follows the general to the specific procedure quite naturally as well.

The above questions and replies are all about goods. As AW mentioned earlier, the Act captures both goods and services, so it follows very naturally to talk about services next. Therefore the dialogue now proceeds from the general to the specific, from the whole to the part. In fact IH here also re-mentions guarantees about service as a new part to the Act before he asks "what is a service" (the nineteenth turn), in order to provide a structural transition. AW's reply covers the points, which become IH's question topics as what happens in the talk about goods guarantee, i.e. "reasonable skill and care" (the twentieth turn), "fit for a particular purpose" (the twenty-first turn), "reasonable price" (the twenty-second turn), "completed within a 
reasonable time" (the twenty-third turn). This service guarantee part is obviously also structured by a movement from the general to the specific, from the whole to the part. Interestingly the last two questions are also presented in illocutionary metonymies, using statements to metonymically pose questions (i.e. ASSERTION FOR QUESTION), e.g. "and a reasonable price" for "what do we mean by a reasonable price?" and "the hope that it will be completed in a reasonable time" for "what do we mean by being completed in a reasonable time?" Those utterances containing "what do we mean" are metonymic as well. Specifically it might be WE FOR THE ACT, a SUBJECTIVE FOR OBJECTIVE metonymy, or WE FOR YOU, a FIRST PERSON FOR THE SECOND PERSON metonymy

In fact, this kind of metonymy has specific discourse functions in interviews. It can first help structure and develop the interview, as analysed above. Second, it helps the interviewer create a friendly or intimate atmosphere for the interviewee, making the interviewee more open-minded and thus obtaining more specific and significant information from the interviewee. Because interviews are in the form of questions and replies, it is very common to produce an embarrassing moment if the interviewer is not cautious in posing his/her questions and in employing his/her tone.

The schematic progression by which metonymy structures and develops the above interview may be clarified as follows (Figure 8.1): 


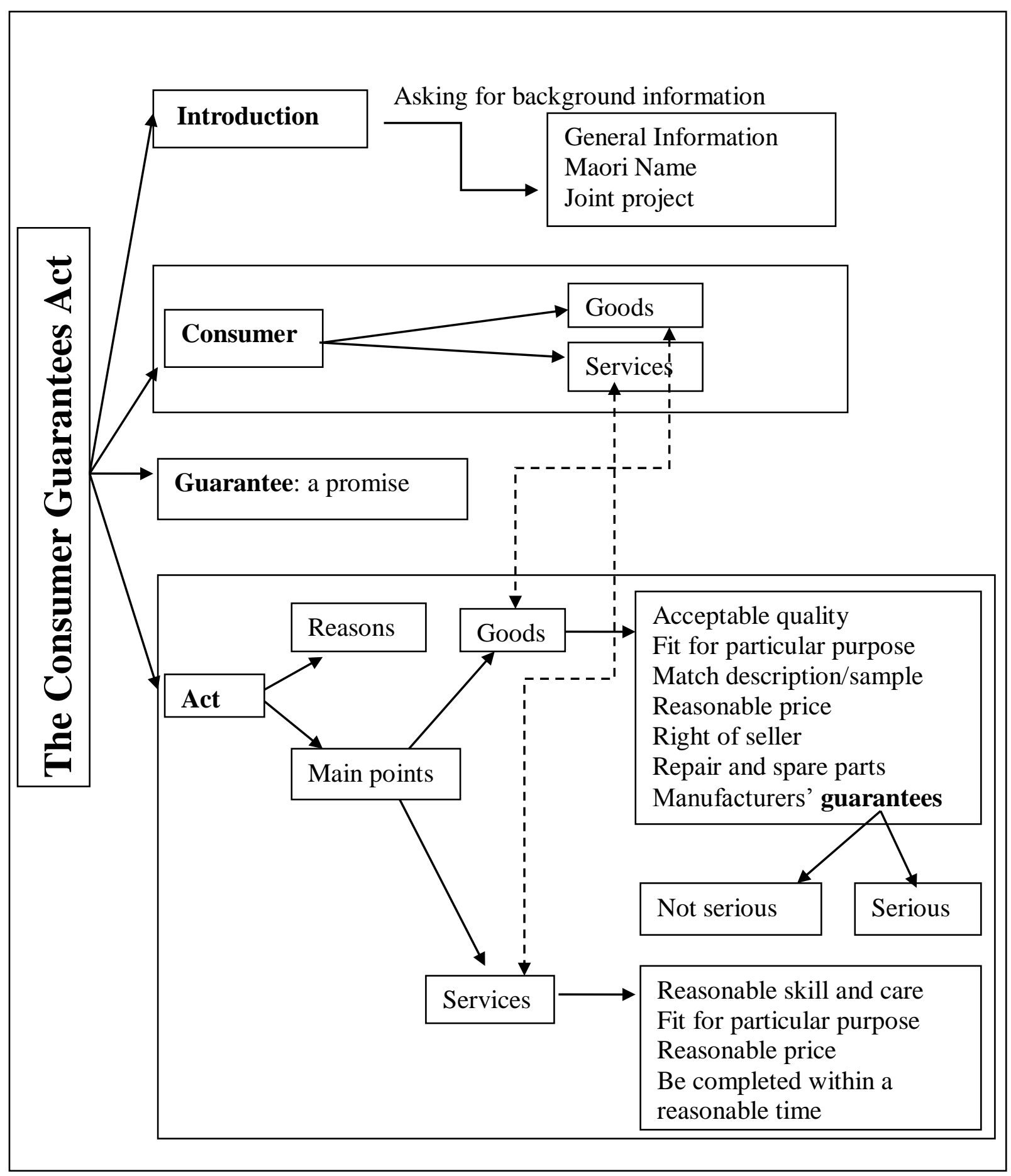

Figure 8.1: How metonymy structures and develops WSC\#DGI158

This diagram shows how interviews can be developed and structured with the help of metonymy. The underlying process is overall GENERAL TO SPECIFIC, but there are still other forms of metonymy, e.g. ASSERTION FOR QUESTION illocutionary 
metonymy, and PART-PART RELATING (see the dotted arrows). This interview is in fact not complete; however, it does not prevent the readers from inferring that it will move on to questions about service complaints or negative services. This is the power of metonymy in structuring and developing dialogic discourse in general, interviews in particular. The GENERAL TO SPECIFIC procedure of metonymy allows people to have reasonable expectations and predictions.

\subsection{Summary}

Following the conceptual view of metonymy as a natural cognitive mechanism involving PART-WHOLE FORMING, PART-WHOLE RELATING, and PARTPART RELATING processes, this chapter considers metonymy and dialogic discourse from the conceptual perspective. Metonymy in discourse has been researched by the cognitive approach, but the focus is generally placed on words in a sentence; when dialogue is considered, the focus seems to rest on isolated sentences or single talk-turns, or mainly on speech act metonymies. The function of metonymy in structuring and developing discourse has been touched on to some degree, but the concentration has been mostly placed on the role it plays in the coherence and cohesion of neighbouring sentences, on some relations between words within a sentence or between sentences such as anaphora (Markert \& Hahn, 1997; Ruiz de Mendoza \& Díez Velasco, 2004; Stirling, 1996; Warren, 2004), on PART-WHOLE, GENERAL-SPECIFIC relations $^{78}$, and on narratives, e.g. AI-Sharafi (2004).

Due to the metonymic nature of Embodied Cognition, human thinking is likely to be essentially metonymic. The commonly used types of reasoning also seem to be based on metonymic operation in categorization and conceptualization. Therefore, the structuring and developing function of metonymy is not limited to words between neighbouring sentences, nor limited to narrative texts or discourse. One of the most common forms of human communication is dialogue. In dialogic discourse, metonymy underpins the dialogue proceeding in various forms.

\footnotetext{
${ }^{78}$ The metonymy literature has mentioned GENERAL-SPECIFIC and SPECIFIC-GENERAL, but they are put forward as the relations after the WHOLE (GENERAL) has already been established. This is different from what is claimed in this thesis, i.e. like conceptualization, perceptualization, and categorization, the PART-WHOLE FORMING still operates in dialogic discourse, as shown in the first interview instance.
} 
The first interview illustrated that sometimes the interviewer comes to a general view from the specific case that the interviewee talks about, and then from the general point the interviewer presents another question for specific information or more details. This is the interaction of PART-WHOLE FORMING and PART-WHOLE RELATING processes. In some cases, the latter process dominates the dialogic discourse, as in the second interview. But more frequently these two processes interact in dialogic discourse, together with PART-PART RELATING process. Relating a PART to the WHOLE is based on the fact that the whole has already been formed, which is often beyond consciousness and thus easy to overlook. What is examined here is the structuring and developing function of metonymy underlying the interviews as a dialogic discourse. It might be PART-WHOLE RELATING process (and its various subtypes) that plays the vital role. But if the interviewer is taken into account, it might be a PART-WHOLE FORMING process in general. For example, in the second interview, IH might have has formed a rough model of the Act under discussion, that is, he may have had a framework of the Act in terms of information before the interview; but it is a rough one. During the interview, IH is also developing and enriching his own knowledge model of the Act (a WHOLE) as he elicits additional information on it from $\mathrm{AH}$ and thus his own model gradually clarifies. In this sense, the PART-WHOLE FORMING process is right under way. He then uses the information from $\mathrm{AH}$ to focus on further specific aspects of the Act, i.e. the PART-WHOLE RELATING and PART-PART RELATING processes.

This chapter only considered the structuring and developing function of metonymy between talk-turns in dialogic discourse, but in fact metonymy also underlies a long talk-turn in both of the two interviews, because the speaker sometimes develops his/her talk in some form of the metonymic mechanism. Take a part of the second interview for example. In AW's eighteenth turn, she mentioned what to do if there were serious faults of goods "cancel the contract/return the goods", and "get the money back". Following this, AW goes further to talk about the consequential loss or any extra loss in both these instances (i.e. both non-serious and serious faults), which involves two situations: One situation is to seek the consequential loss or extra loss from the seller, the other situation is that the buyers can even seek the loss from the manufacturer under the new Act. It is clear that AW is developing her talk further from the general to the specific (faults - what to do - loss from the fault - what to 
do). This happens within the scenario of fault goods, it is accordingly a PART-PART RELATING; but the relation between the parts here is GENERAL-SPECIFIC. It seems possible that GENERAL-SPECIFIC relation does not limit to the WHOLE and its parts, but also occurs between its parts.

For dialogic discourse, talk-turns are the key points in terms of structure and development. This is why the analysis is focused on metonymy underlying those turns. It seems that in interviews the interviewer generally guides the interview, e.g. posing questions (including ASSERTION FOR QUESTION) to the interviewee. Nevertheless, as a discourse, the interviewer also develops his further questions or topics from the information the interviewee provides. In this sense, the interviewee contributes to the structuring and development of the dialogic discourse as well. Apart from the metonymies such as FACT FOR REASON and ASSERTION FOR QUESTION that motivate the interviewee's utterances, the interviewee's own contribution to the structuring and development of the dialogic discourse is also an interesting area to study. Also it is significant to examine how metonymy functions within each long talk-turn in terms of structure and development, from both sides of the interviewer and the interviewee. 


\section{Chapter 9 Metonymy in Dialogic Discourse: Meaning- making, Decision-making, and Problem-solving}

The preceding chapter examined, from the conceptual perspective, metonymy in dialogic discourse in general, and in interviews in particular illustrating how metonymy structures and develops interviews. The structuring function of metonymy in interviews is evidenced through analysis of talk turns, especially in how the interviewer metonymically presents questions and asks for information. Another aspect of metonymy in dialogic discourse, specifically in interviews, is whether metonymy operates in the interviewee's replies; if so, how does metonymy contribute to the problem-solving (i.e. answering the question or solving the problem posed in the interviewer's talk-turn)? The other aspects related to problem-solving are meaning-making and decision-making, i.e. how metonymy helps make meanings out of the dialogue at the discourse level and make decisions within the dialogue on behalf of both the interviewer and the interviewee. These three aspects are focused on in this chapter.

\subsection{Metonymy and meaning-making}

In order to speak of meaning-making, another similar notion, meaning construction, has to be mentioned and differentiated. As argued in Chapter 6, meaning construction is a view that considers meaning as dynamic and as having to be constructed online, whether it is word meaning or sentence meaning, through some mechanisms such as metonymy, metaphor, mental space and conceptual blending. Thus meaning construction is "an on-line mental activity whereby speech participants create meanings in every communicative act on the basis of underspecified linguistic units" (Radden, et al., 2007a), see also Evans \& Zinken (to appear), Evans (2006, 2009, 2010b). To put it more clearly, the notion of meaning construction denotes the pragmatic reasoning leading to the determination of explicit meaning (explicature) and implicit meaning (implicature), and this reasoning is guided by natural inference schemas largely provided by metonymy (Barcelona, 2007b; Panther, 2005). 
Meaning-making has variant understandings as used in different disciplines, however. In the social sciences, it essentially means that people impose meaning on the world according to their own experience, goals, action, motivation, etc (Kurzman, 2008). People understand the world in their own way and make meaning out of their lives and experiences, i.e. they draw meanings from, or give meanings to, events and experiences (Krauss, 2005). In some fields, e.g. cultural heritage, meaning-making means knowledge construction (Hein, 1999). In psychology and other fields, meaning-making indicates meaning detection and creation (Wilson, 2010), and is even considered as "the ability to integrate challenging or ambiguous situations into a framework of personal meaning using conscious, value-based reflection" (Schreurs, et al., 2009, p. 510). In linguistics, meaning-making is used sometimes with similar sense as meaning-construction, or meaning creation, or rather, it is treated as sensemaking in the general sense, e.g. Scott et al (2006), Benczes (2009).

Within this general linguistic framework, meaning-making in this chapter is employed in a more specific way. Regarding metonymy and meaning-making in dialogic discourse, meaning-making is used here with a different interpretation. When metonymy or metaphor is connected with meaning-making, it usually suggests how a word, a phrase, or a sentence is meant through the mechanism of metonymy or metaphor, and how it is supposed to be understood. Here in the analysis of metonymy in dialogic discourse, meaning-making means how metonymy (i.e. a metonymic utterance) contributes to the whole dialogic discourse, not merely how the metonymic utterance makes sense locally. This function of metonymy has in fact been identified in Cognitive Linguistics, especially in the study of metonymy-based inferences at the text level (see e.g. Barcelona, 2007b). An analysis of the examples below can help illustrate this point:

\begin{tabular}{|l|l|}
\hline Amelia Bly: & $\begin{array}{l}\text { We need another pair of hands, but Adam couldn't } \\
\text { bring himself to replace Mike. They were together so } \\
\text { long. }\end{array}$ \\
\hline Ghostwriter: & How long were you with him? \\
\hline Amelia Bly: & Eight years. I am an attachment from Number 10. \\
\hline Ghostwriter: & Oh, poor Number 10. \\
\hline & \multicolumn{2}{r}{ Ghostwriter, 2010 (Movie) } \\
\hline
\end{tabular}


The above dialogue evidences some metonymy-motivated examples of language use, i.e. the boldfaced parts. This is a dialogue in the movie Ghostwriter between the British Prime Minister Adam's assistant Amelia Bly and the newly hired ghostwriter, in which pair of hands means writers (ghostwriters, to be specific), attachment means assistant, the first Number 10 means 10 Downing Street, i.e. the British Prime Minister's Office, but the second Number 10 means the British Prime Minister. Of course these metonymy-motivated words, or to put it more simply, these metonymies, means more besides the above mentioned, i.e. they carry a specific perspective, according to the analysis of metonymy as a cognitive mechanism. For instance, pair of hands means writers, focusing on the perspective of hand use; attachment, focuses on the referent's (secondary) status; Number 10's, though with different references in this dialogue, both focus on the location of the referent. All these interpretations are thus meaning-making merely at the local level, not at the discourse level, because the interpretations of this kind don't contribute to the dialogue as a whole entity. The meaning-making at the dialogic discourse level can be shown in The Devil's Own example (to follow).

In some situations the local meaning-making of metonymy speaks. There are three situations in dialogic discourse where metonymy performs the local meaning-making. The first is that the speaker makes a new metonymy-motivated expression which is totally novel to the listener, for instance:

A Wellyworder ${ }^{79}$ (W, for short) called Telecom and had a lovely chat with the man at the desk (D, for short).

\begin{tabular}{|l|l|}
\hline W: & What are your plans for the weekend, then? \\
\hline D: & Oh, I'm working, and then I have my honeydo list. \\
\hline W: & Honeydew list? What's that? \\
\hline D: & You know, 'Honey, do this...', 'Honey, do that....' \\
\hline \multicolumn{2}{|}{ Adapted from Capital Times 8-14 June, 2011. P.3 } \\
\hline
\end{tabular}

\footnotetext{
${ }^{79}$ There is a column called Wellyword in the Capital Times, a local newspaper in Wellington, which talks about funny stories or experiences of some people in Wellington. These people are named Wellyworders in the column.
} 
In this instance, the metonymy-motivated compound honeydo has a local meaningmaking function, but it is new to the listener. Therefore he could not understand it and even misunderstood it as honeydew according to his own knowledge and experience.

The second type of local meaning-making is where the speaker uses a metonymymotivated expression which is known to some or most people in a certain situation (e.g. the speakers of the language as their mother tongue, or people in a particular field, group, class, etc) but is new to the listener. Here is an example excerpted from ICE-Singapore:

\section{ICE-SIN:S1A-003}

232:A

I know that I'm going to Thailand so I didn't want to shop 233:A

I wasn't shopping for the last three months or so two three months 234:C

So you just let it out in the big bang 235:A

But I didn't buy much because I was quite disappointed with the clothes 236:A

I was thinking of buying accessories and but then I was quite reluctant to part with one hundred dollars just for things like that

237:C

What's the in thing there

238:A

\section{In thing}

239:C

at the moment

240:A

in thing I'm not quite sure

241:A

What is in thing

242:C 
I mean like the clothes what's what's in fashion lah

243:A

Couldn't really identify any fashion

244:A

It's all the long sum

245:A

Maybe that's the in thing now

246:A

I couldn't see cotton material

247:A

Quite plain it's just blouse

248:C

And that's it

249:A

$\mathrm{Ya}$

In this dialogue, $\mathrm{C}$ makes use of a metonymy-motivated expression in thing, which might be well known to native speakers of English, but here it seems to be totally a strange expression to A. So in this situation, this metonymy is only making a local meaning out of the utterances, it does not contribute to the dialogue as a whole discourse.

A third instance is when the speaker makes use of a common expression but with his own interpretation in the dialogue, for instance (also excerpted from ICE-Singapore):

ICE-NZ:S1A-010

$1: \mathrm{D}$

my brother's going to turn into a street kid

$2: \mathrm{H}$

your brother?

$3: \mathrm{D}$

yeah

4:D

oh you know I said he was leaving school

$5: \mathrm{H}$ 
oh yeah

6:D

well he's left school

Street kid here in D's utterance does not mean the same as in the dictionary, i.e. a homeless kid, but refers to a created meaning as a kid who has left school. That is why $\mathrm{H}$ found it unbelievable (“your brother?").

All these situations are where metonymy has a local meaning-making function. But this function is not the focus in this analysis of metonymy in dialogic discourse. Viewed from the (dialogic) discourse perspective, metonymy contributes to the dialogic discourse as a whole entity or event scenario as well. Let's see the following example:

\begin{tabular}{|c|c|}
\hline $\mathrm{R}:$ & Mr O’Meara? \\
\hline $\mathrm{T}:$ & Tom. \\
\hline R: & Hi. \\
\hline $\mathrm{T}:$ & Come on in. That's Amie \\
\hline $\mathrm{R}:$ & Hi, Amie. \\
\hline $\mathrm{T}:$ & How was your trip? \\
\hline $\mathrm{R}:$ & It's good. \\
\hline $\mathrm{T}:$ & A long one, isn't? \\
\hline $\mathrm{R}:$ & Aye. \\
\hline \multicolumn{2}{|r|}{$\begin{array}{l}\text { (Walking into the house, introducing Rory to his daughters and his wife } \\
\text { Sheila) }\end{array}$} \\
\hline $\mathrm{T}:$ & $\begin{array}{l}\text { Er, come on in. This, er this is Morgie. Morgie, Morgan, hey, honey, } \\
\text { say Hi to Rory. (Morgie waves her right hand to greet Rory with a } \\
\text { smile) }\end{array}$ \\
\hline $\mathrm{T}:$ & Sheila is in here. Say hello. \\
\hline $\mathrm{T}:$ & Sheila. \\
\hline S: & Yeah. \\
\hline $\mathrm{T}:$ & Rory is here. \\
\hline
\end{tabular}




\begin{tabular}{|l|l|}
\hline S: & Hi, welcome. I am the mother \\
\hline R: & Nice to meet you. \\
\hline S: & Thanks. \\
\hline T: & Where is Bridget? \\
\hline S: & What do you think? She is on the phone. (the kettle is boiling) Oh. \\
\hline T: & Careful. \\
\hline T: & $\begin{array}{l}\text { The kids were supposed to get this crap out of the hallway. (going } \\
\text { down to the basement) }\end{array}$ \\
\hline T: & Well, it's not so much, but it's dry and it's warm. \\
\hline T: & The bed's over here, there is a bit of bath and shower here. \\
\hline T: & You've got to jiggle with this thing. Sometimes it runs on. \\
\hline T: & How are you fixed for work? Did Fitz find something for you? \\
\hline R: & I start in a couple of days. \\
\hline T: & Good. The TV's here. What are you doing? \\
\hline R: & A building site \\
\hline T: & Construction? \\
\hline R: & Aye. \\
\hline T: & Good, good, good. \\
\hline T: & Well, if there's anything you need ... \\
\hline R: & No, no, it's ok. Very decent of you for putting me up like this. \\
\hline T: & Don't thank us. Thank Fitz. \\
\hline S: & Guys, dinner! \\
\hline T: & $\begin{array}{l}\text { We'll be right there, honey. Besides, it's good to have somebody } \\
\text { around here that pees standing up. }\end{array}$ \\
\hline & \\
\hline & \\
\hline
\end{tabular}

This is a transcription of a scene in the movie The Devil's Own, which describes the moments when Frankie (who went to USA and lived in Sergeant Tom O'Meara's house under the false name "Rory Devaney") went to Tom's house for accommodation already arranged by Fitz (Peter Fitzsimmons, a New York court judge), and Tom showed him around introducing him to his family (three daughters and his wife), and led him to the basement where he will live. 
First let's see the first boldfaced sentences. These involve metonymies. In Tom's turn, the first question (“How are you fixed for work?") employs a general (idiomatic) expression for the question "do you have a job?" And the second question ("Did Fitz find you something?") also uses a very general word "something" to metonymically refer to a job or any work, which is very common. They involve the GENERAL FOR SPECIFIC metonymy. Rory's answer ("I start in a couple of days.") is also a metonymy in that it uses the AFTER component, in Panther's term, to indicate the CORE, i.e. He has found a job for me. Simultaneously his reply employs another metonymy: Using the present tense for the future, i.e. PRESENT FOR FUTURE. It may be said that the metonymies such as SOMETHING FOR A JOB, AFTER FOR CORE, PRESENT FOR FUTURE are all meaning-making in the narrow sense, that is, they are helpful in understanding what is uttered. But this is not enough in the current analysis. The meaning-making of metonymy here, e.g. the PRESENT FOR FUTURE metonymy and the AFTER FOR CORE metonymy, rests on its contribution to the dialogue as a whole discourse, to the event as a whole (i.e. living in Tom's house). For example, the metonymic use of "something" suggests that Tom is not sure what Rory is going to do on the one hand, and on the other hand, it implies that Tom cares about Rory in terms of making a living. Firstly the AFTER FOR CORE metonymy and the PRESENT FOR FUTURE metonymy are the positive answer to the question, and secondly they suggest that he will start a new job very soon and thus settle down easily. Taking all these into account, it can be easily inferred that: Yes, he found me a job and I will start in a couple of days on the one hand, and on the other hand, he employs another metonymy, i.e. he uses the present tense to mean the future, to imply that everything is favourable and he is eager and keen to do the job. That is to say, these metonymies suggest, in Rory's perspective, "Everything is going favourably and I have a job already, I feel happy to start and can settle down easily, please don't worry about me, even if this is my first time in America".

In the following dialogic sequences, Rory's reply "A building site" presents another metonymy. He uses a very general phrase of whereabouts to avoid giving specific information about what he is doing. This metonymy functions as meaning-making in the sense that it contributes to the whole storyline. In fact, he is going to do repairing work on a ship they bought to carry missiles to Ireland, and his working place is near 
a building site. Therefore, his metonymic reply, first, is not a lie to Tom, and second, it avoids specific information about exactly what he is doing and where it is. When Tom metonymically concludes "Construction”, Rory just answers “Aye”. Here again, Tom's uncertain interrogation comes from the SPECIFIC-GENERAL metonymy, i.e. "a building site" activates the general CONSTRUCTION concept, which takes place within the CONSTRUCTION category ( the WHOLE), thus it is the PART-WHOLE RELATING process that is involved here, with a structuring function in the dialogue as well.

The last example is "Besides, it's good to have somebody around here that pees standing up". This utterance comes after Tom's wife Sheila calls them for dinner and before they come up to dinner, and it is more like a concluding remark to Rory in this introductory scene. Here again, a metonymy is involved, i.e. "somebody that pees standing up" for "a man" ((SPECIFIC) FEATURE FOR THOSE WHO HAVE THIS FEATURE). If only understood this way, this utterance is not meaning-making as employed here. The meaning-making of this metonymy as used here is a heartfelt welcome to Rory. It expresses Tom's cordial reception and best hospitality. From the scene, it is known that Tom is the only man in his family of five members, but now comes another man: Rory. In Tom's view, it is beneficial to his domestic situation. In addition, this metonymic utterance functions as a gesture of solidarity in a humorous manner and makes Rory feel at home in a new house in the new country. This inference is probably guided by an EFFECT FOR ATTITUDE metonymy ("it is good that you are here with us" for "you are really welcome here"). The meaningmaking here may be missed if the analysis of metonymy is confined to isolated sentences or to the local interaction. This is precisely the meaning-making function of metonymy in dialogic discourse: it contributes to the whole scenario at the macrolevel and helps, from the perspective of dialogic discourse, to uncover and clarify what is locally veiled or missed.

\subsection{Metonymy and decision-making}

This section will examine whether metonymy operates in decision-making in dialogic discourse, and if so, how it functions. In the above The Devil's Own example, metonymy does operate and help make a final decision on what to give by 
way of an appropriate reply. For instance, for the sake of analysis, the part is cited here again as follows:

\begin{tabular}{|l|l|}
\hline T: & How are you fixed for work? Did Fitz find you something? \\
\hline R: & I start in a couple of days. \\
\hline T: & The TV's here. What are you doing? \\
\hline R: & A building site \\
\hline T: & Construction? \\
\hline R: & Aye. \\
\hline
\end{tabular}

As the above analysis has mentioned, Tom has to decide how to ask about what Rory is going to do once he arrives in America, living in his house as arranged by Fitz. He has many alternative ways of presenting the question, for example: What is your job? What do you do? What's your plan? Did Fitz find you any work? Did Fitz find you something to do? But, in Tom's view, none of these choices is ideal for a new arrival, in case it makes him embarrassed and ashamed. Asking about work actually forms a scenario, which can be triggered and accessed by different components or aspects. After weighing the situation ${ }^{80}$, deliberately or unconsciously, Tom chooses a very general expression to serve both his need for information and his sense of politeness.

Rory also has many alternative replies, for example: "Yeah, he has found me a job"; "He has an arrangement"; or just answer "Yes". However, in spite of these choices, Rory gives a reply that best fits the situation and the discourse scenario: "I start in a couple of days". He even does not use will in his reply. The reply and the PRESENT FOR FUTURE metonymy in this utterance not only give a positive answer to the question, i.e. "Fitz has found me a job", but also give Tom the impression that all has been well scheduled and "I am just acting according to the schedule." And also this choice allows Rory to avoid specific information about what he will do, perfectly corresponding to Tom's question.

But Tom seems to want to have an idea of what Rory is going to do, so he puts forward another question: "what are you doing?" Here this question is not following

\footnotetext{
${ }^{80}$ Of course, there are some other factors functioning as well when weighing the situation in personal communications, such as cooperative principles (Grice, 1991), relevance principles (Sperber \& Wilson, 1995), politeness conventions (Brown \& Levinson, 1987(Brown \& Levinson, 1987), and other factors concerning the speaker's interests, goals, attitudes, etc.
} 
Rory's answer: "I start in a couple of days", directly, i.e. this question is not something like what do you do to correspond with Rory's use of present tense. Rather, Tom uses the PRESENT PROGRESSIVE FOR FUTURE metonymy to correspond with Rory's PRESENT FOR FUTURE metonymy, and to carry more information, i.e. both the present and the future, indicating something like, "I take the point that you have a job already but what is it or what will you do on the job?"

As already analysed in section 9.1, Rory's reply: "a building site", is also a final decision from other possible choices based on metonymy, e.g. "I am doing construction work", "I am doing the design work on a building site", "I am doing architectural work", or just "I am an architect or designer", etc. But Rory does not mean to tell lies, thus the more general the answer is, the better it is. His reply "a building site" is precisely where he will be working: Repairing the ship near the site. It is a LOCATION FOR WORK TO BE DONE THERE metonymy; or in other words, the metonymy LOCATION FOR WORK underlies his response. Therefore, it is obvious that metonymy is operating to help him make a decision and give an appropriate reply.

By the same token, Tom's consequent question "construction?" also comes among alternatives, such as "A building site?" "What you do exactly?" "Is it far?" "Where is it?" Based on building (site), Tom just asks a question comprising a very general term. That is, it involves a PART-WHOLE RELATING process (BUILDING SITE TO CONSTRUCTION). The decision of using the general term through metonymy suggests that it is not the case that Tom really wants to know what exactly Rory will do, but it is an indication of Tom's considerate treatment of and concern for the new family member.

In dialogic discourse the decision-making role of metonymy rests on the interactive exchange of utterances between the communicators, i.e. how through metonymy the speaker arrives at his utterance among many alternatives. This metonymic utterance is surely contributory to the dialogue as a whole discourse. The questions and the answers in dialogic discourse are the best place to evidence the decision-making function of metonymy. Metonymy is ubiquitous in language and cognition, and hence so is the decision-making function of metonymy. This is in fact not difficult to comprehend. The arguments in the previous chapters suggest that metonymy, 
whether in conceptualization, categorization, memory, inference, or in scenarios, always highlights a specific perspective of an entity, it leads to a whole based on a particular part, or section, or aspect, i.e. PART-WHOLE FORMING. It also guides memory and inference once the whole experience is patterned and stereotyped, and enables the PART to speak for the WHOLE or the PART to speak for other PART(s), i.e. PART-WHOLE RELATING and PART-PART RELATING. Whenever a question is presented or answered, the utterance might be seen as the final decision made among choices, most likely through the cognitive mechanism of metonymy, and at the same time it is only one part or aspect of the whole picture or related experience pattern. This cognitive feature derives from the inherent nature of embodied cognition, which has been explained in Chapter 3 and will not be detailed again here. But the full relevance to decision-making can be made clear by examining another example here from ICE-NZ: S1B-049, which is an interview between I (a male radio broadcaster) and M (a Maori male community worker) about Maori development in New Zealand (please see the CD attached).

The first talk-turn in this dialogue is a kind of introduction of $\mathrm{M}$ to the audience, simultaneously leading to the interview topic. As there are many ways to serve this function, I has to make a final decision as to what will be a satisfactory opening. I's utterance presents $\mathrm{M}$ as an expert in Maori development and at the same time introduces the organization he works for, paving the way well for the general interview topic about M's views on Maori development. This decision-making involves at least BEING/WORKING WITH AN ORGANIZATION FOR EXPERT IN WHAT THE ORGANIZATION DOES. After M gives a positive answer, I leads the dialogue further to a specific aspect of the general topic, i.e. the 1991 Post Budget Period (the second turn), asking for M's view of the impact it will have on Maori people. I asks a selective question and a what question. His first sentence in the turn involves a LOCATION FOR RESULT metonymy ("about where we're at nineteen ninety one post budget"). And the later two sentences involve EFFECT FOR ATTITUDE ("positive", "gloomy”) and ATTITUDE FOR EFFECT ("view", "perspective") respectively. That is to say, I's second turn involves a metonymic chain: LOCATION FOR RESULT - RESULT FOR EFFECT - EFFECT FOR ATTITUDE - ATTITUDE FOR EFFECT. It is the natural inferential schema of metonymy that is involved here. M's reply is that it is good for Pakeha people and 
worse for Maori people. He puts forward this opinion through specific media readings, i.e. he overall gives his utterance and opinion through PART-PART metonymy (Pakeha people-Maori people) together with comparison. Some other metonymies are also involved in M's reply. For example, MEDIA VIEW FOR PAKEHA VIEW (media commentators' view represents Pakeha people's view), MEMBER FOR CATEGORY ("we" for "the nation" (\#6), "we" for "Commission for Justice Peace and Development" (\#9), and "we" for "Maori" (\#11)).

Based on M's specific cases of Pakeha and Maori, I forms a conclusive remark in his third turn: "This is a function of our political parties not being interested in the Maori people". This process is likely to involve INDIVIDUAL FOR THE PUBLIC metonymy, which can be supported by his quietly added words "I think". M's reply of "personal observation", to some degree, might be expected to prevent I's INDIVIDUAL FOR PUBLIC interpretation. But M's personal observation is based on what he is hearing and seeing. Here it at least involves HAPPENING TO RESULT (he gets the distinct impression from his observation that the fundamental changes taking place are bolstering the position of settlers), in addition to the SPECIFIC TO GENERAL process (what he is hearing and seeing - impression overall view). There is also a HAPPENING TO RESULT TO EFFECT/OPINION metonymic chain underlying M's reply. What is happening in the society naturally leads to this opinion. I's utterance (the third turn) can be considered as the EVALUATION $^{81}$, and $\mathrm{M}$ is emphasising that it comes from the HAPPENING. This underlying metonymy therefore triggers I's next question (the fourth turn) "how the hell did this happen?" They are trying to trace the REASON (or the BEFORE component, in Thornburg and Panther's term) for the HAPPENING. So M's EVALUATION and HAPPENING force I to decide to ask about the REASON. Then $M$ starts to talk about the REASON for the HAPPENING. Through talking about the general HAPPENING, M arrives at the EVALUATION: This is a cultural conflict between the settlers (Pakeha) and the Maori. All the happenings suggest that the country is promoting individualism, but Maori people give more value to the

\footnotetext{
${ }^{81}$ The scenario of asking for somebody's views on something generally involves HAPPENING (what is happening or has happened), CONSEQUENCE (what is the consequence the happening brings), and EVALUATION (the effect of the consequence upon people, i.e. what people think of it). They can be considered similarly as Thornburg and Panther's CORE, RESULT, and AFTER components in action scenarios.
} 
whole group and thus have divergent attitudes and behaviour. Therefore, through talking about the HAPPENING, general or specific, $M$ is led naturally to present the REASON.

The HAPPENING and REASON M puts forward furthers I's interrogation: Since we have a constitution and have advanced so much, why does this still happen (the fifth and sixth turns)? Through examining the development of the society, i.e. the HAPPENING, I arrives at the REASON: The government did not pay attention to Maoridom economically. Following I's turn, $\mathrm{M}$ starts to talk about the further REASON behind the HAPPENING. Also through talking about the actual happenings and specific cases, $M$ comes to the deeper REASON: "In the past years, it is constitutional and legal matters that have been focused on, with the economy out of sight, thus there is no provision made for a sharing of wealth and power in this country." It is clear that M makes up his mind to give the REASON through citing the HAPPENING (actual cases), and this HAPPENING FOR REASON makes his view sound more objective, reasonable, and well-grounded.

The root cause of the reality made explicit by $M$ falls within the responsibility of another organization (the Royal Commission on Social Policy), and this provides I with the cognitive access to the commission $\mathrm{M}$ worked for before (this comes from I's knowledge of the organization, but it is also possibly related to the interviewee M). This makes I decide to talk about the goal of the commission (the seventh turn). M continues I's talk, citing actual cases (HAPPENING), e.g. the Royal Commission on Social Policy, the churches, community groups are all addressing the wealth and power sharing, and comes to the point that those in control of the political parties have not been taking Maori people seriously, though many other groups and institutions have been addressing the problem.

Regarding the past governments and wealth and power sharing mentioned in M's turn, I decides (the eighth turn) to ask about the previous Labour government's stance towards economic sharing between Pakeha and Maori. The talk of past governments naturally activates the present government, thus it may undergo a PAST TO PRESENT process. Therefore his question focuses on what this government has done in the past few days and what they can do in this respect in the coming years. In order to spell out the seriousness of the problem, $\mathrm{M}$ decides to first 
give a general idea of what approach both the main political parties make, i.e. monetarism, and then give detailed information on what monetarism is (reply to I's ninth turn) and how its proponents operate, in contrast to a people-oriented approach, i.e. egalitarianism (everybody should have equal access to resources). He then comes to the specific situation where Maori were only the servants of someone else's economy even under the past egalitarianism, concluding that there is almost no Maori private sector. In his talk, $\mathrm{M}$ is proceeding from the general to the specific and then to the general again in terms of HAPPENING (Maori people were serving the interests of non-Maori) and CONSEQUENCE/RESULT (no Maori entrepreneurs) and REASON (we don't have many of those people who start businesses creating jobs for Maori and so on). This then triggers I's talk about the further CONSEQUENCE $^{82}$ (i.e. it resulted in the consequence that those talented Maori people who could become entrepreneurs had all gone overseas) (the eleventh turn) and the REASON. But I does not in fact spell out the REASON by just saying "they got sick of the bullshit here and went over there to have a go" (the twelfth turn), this is more like a RESULT FOR REASON. This is why $M$ follows I's talk of the RESULT but not the REASON right away in his next turn. M cites the RESULT (e.g. in Sydney, Los Angeles), and then comes to the REASON: "why couldn't they do that in New Zealand when they are successful businessmen in America and Australia?" M continues to cite HAPPENING (people said that they found it difficult to get money from the bank) and then comes to the REASON (an entrenched racial prejudice) and the CONSEQUENCE (lack of commercial enterprise), from New Zealand to the general Pacific area.

The above analysis shows that the speakers present their ideas through the various forms of metonymy. Apart from those, the common forms GENERAL TO SPECIFIC and SPECIFIC TO GENERAL also underlie their decision-making: From specific cases to a general conclusion or vice versa. They present their ideas in

\footnotetext{
${ }^{82}$ In the continual progression of an event, the REASON can also become a new HAPPENING/ CONSEQUENCE, and the CONSEQUENCE can become in turn a new HAPPENING. For instance, here in this interview, the HAPPENING (Maori people were serving the interests of non Maori) can also be taken as both the CONSEQUENCE of the previous government policies that may be also taken as the original or real HAPPENING and the REASON for the CONSEQUENCE (No Maori entrepreneurs); and simultaneously, this CONSEQUENCE M talks about (No Maori entrepreneurs) and the further CONSEQUENCE I talks about (the talented go getters have gone to Australia), which can be also taken as the HAPPENINGs at that time. Therefore, a HAPPENING can be both the CONSEQUENCE of previous HAPPENINGs and the REASON for a new HAPPENING in a continual event scenario.
} 
whatever be the most efficient and effective way. Each utterance or talk-turn is a decision made by the speaker, for the sake of effective expression of their ideas and opinions. From the speaker's talk, especially the interviewee's utterance, it can be seen how metonymy helps organize his talk and decide what to talk about among alternatives. But to make sense of the decision-making of metonymy in dialogic discourse, a dialogic discourse perspective needs to be taken, considering the utterance of each talk-turn within the framework of Event Scenario (generally involving REASON, HAPPENING, CONSEQUENCE, and EVALUATION, though their function or status can be altered in a continual progression, as pointed out in the previous note). The above analysis also suggests that, although often related, the decision-making, the structuring, and meaning-making functions of metonymy are different aspects of the dialogic discourse in terms of the function metonymy serves as a cognitive mechanism. The structuring and developing function of metonymy rests on how the talk turns are made coherent and contributes to the dialogue/interview as a whole dialogic discourse. The meaning-making function of metonymy builds on the metonymies used in the dialogue which make a discourse level contribution in the event scenario. The decision-making function of metonymy hinges on how the speakers make their utterance through metonymy from choices in general, and organize their utterance metonymically in particular. As to how the interviewee/receiver answers the questions, i.e. solves the problems put forward by the interviewer/speaker, that is another aspect, which is to be addressed next.

\subsection{Metonymy and problem-solving}

As pointed out above, problem-solving and decision-making are often related and combined together; when they are presented separately, different perspectives are attended to and focused on. Decision-making underscores the selection process in the face of alternatives which are presupposed; problem-solving presupposes the existence of some problem and stresses the integrated consideration and analysis of the problem. Problem-solving underlines the final result and is more concerned with the move from a given state to a desired goal state, and the ultimate goal of problemsolving is to overcome obstacles and find a solution that best resolves the issue (Reed, 2000). 
This section tries to find out whether and how metonymy functions in solving problems in dialogic discourse. Problem-solving seems more common and apparent in actual life than in dialogic discourse, e.g. the breakdown of a car on the road, a virus-infected computer. Nevertheless, in dialogic discourse problems are raised and need to be solved in the form of question and answer, e.g. daily conversation, interview. In this situation, the problems or questions are put forward vocally, and solutions are required.

Besides the verbal problem-solving, there is a situation where problem-solving is conducted through metonymy without verbal expression. For instance, there is a widely circulated story with a long history, which tells of a dumb person selling kitchen knives. Since he is dumb and cannot communicate verbally how good the knives are, selling the knives then becomes a problem. What he does is chop some iron wire and then invite the audience to check the blade. The audience find that the knife is still as sharp as new, and they begin to buy the knives. Thus soon the knives are sold out. This is often employed as a classic successful sales example in marketing.

What is important here is of course the metonymy underlying the problem-solving. The dumb seller knows exactly that customers surely want lasting sharp knives, and this make of knife is sharp enough to even cut iron wire and remains as new, thus cutting the iron wire in public is self-evident, which needs no shouting or yelling. Therefore, the problem-solving is at least motivated and guided by the metonymy MEMBER FOR CATEGORY (i.e. KNIVES THAT CAN CUT IRON FOR GOOD KNIVES). His inference is done in the form of inner dialogue, which may be modelled as follows, within the scenario that people buy sharp knives.

A: What kind of knives do people buy?

B: Very good knives, of course.

A: But what kind of knives can be regarded as good?

B: Sharp and difficult to make blunt.

A: How can I make them know my knives are sharp and not easy to get blunt since I cannot talk?

B: Maybe I can try cutting some iron wire and then show them the knives are as good as before the cutting. 
The general knowledge or experience of people buying sharp knives guides his inference, from the general knowledge to the specific (what are regarded as good knives) to more specific (sharp not easy to get blunt), to a further specific aspect (how are knives considered to be sharp and not easy to get blunt). Thus under this metonymic guidance in inference, he reaches the solution. It is also under this guidance that he had an interactive dialogue with the audience. This is a "silenced dialogue" (Delpit, 1988), because inference is generally conducted internally and there is no verbal communication between them. His witty solution is overall reached through an ACTION FOR SPEAKING metonymy.

Some might say that this event is an ACTION IS SPEAKING metaphor, because the dumb seller just does some action to sell the knives. However, all the inferencemaking processes have to be taken into account. As analysed above, he does the actions (cutting and showing) to serve the similar (actually better) function usually served by talking. He is just doing the cutting and showing instead of verbal selling within the framework of the general experience. Considered from the scenario of selling knives, the dumb seller's actions are obviously components of the scenario. It can also be viewed as GENERAL TO SPECIFIC i.e. PART-WHOLE RELATING. His actions activate the buyers' knowledge of good knives. On the other hand, it might be said that there is another metaphor involved here in the silenced dialogue between the customers and the seller, i.e. SEEING IS BELIEVING. When people watch what the seller does, they believe that the knives he is selling are very good ones. Even so, the SEEING IS BELIEVING metaphor takes place after the general knowledge is formed (the knives that are sharp but not easy to get blunt are very good ones) and some other inevitable inference such as the above are conducted. Therefore, this kind of conceptual metaphor seems more like a final conclusion, e.g. TIME IS MONEY, ARGUMENT IS WAR, LOVE IS A JOURNEY, but these metaphors come into being through metonymic inference, and they are more likely to ground on metonymic operations, as argued in Chapter 4.

Below is another example, which comes from the movie Drive Angry (2011). The dialogue occurs on Milton's way to Louisiana. When Piper, a waitress he met a moment ago but has just quit her job due to sexual harassment, drives by in a super car, which is what he needs to get there, he causes it to break down, then introduces 
himself to her for a ride. When Piper cannot do anything about the broken-down car after a check, Milton walks up and has a look.

\begin{tabular}{|l|l|}
\hline M: & That's not so bad. I can fix it, if you like. \\
\hline P: & I would. Thank you. \\
\hline M: & If you give me a lift. \\
\hline P: & I am only going as far as Blackfoot Falls. The trucks stop there. \\
\hline M: & I'd be much obliged. \\
\hline P: & Thank you. Just so you know, I don't pick up hitchhikers. \\
\hline M: & I didn't have my thumb out. \\
\hline & \multicolumn{2}{|}{ Drive Angry, 2011 (Movie) } \\
\hline
\end{tabular}

Through the whole dialogue, Milton takes advantage of the general experience, i.e. when you help fix a car that is broken down, out of gratitude, your request for a lift will be hard to reject. Thus his utterance pays more attention to the conditions, e.g. in the first two talk-turns, Milton uses two conditionals to present his offer and his desire, i.e. a CONDITIONAL FOR ACTUAL metonymy (of course, he knows exactly that Piper needs help to fix the car, otherwise she will have to abandon the super car at the road side and ask for a lift herself; that is why she answers "I would. Thank you"), and a CONDITIONAL FOR REQUEST metonymy (please give me a lift). Piper's reply to Milton's request for a lift involves metonymic inference as well. She does not directly say yes and she knows she is reluctant to refuse him, therefore she simply says she is going to Blackfoot Falls and then he can take the trucks. In Panther's terms, this is the RESULT component for the CORE (I give you a lift) in the REQUEST scenario, more specifically, in the scenario of ASKING FOR A LIFT. In this situation, the scenario involves the BEFORE (my car is broken down and you help fix it and ask for a lift), the CORE (I give you a lift), the RESULT (I take you to Blackfoot Falls), and the AFTER (you are there). Thus following this, the second sentence in Piper's talk-turn involves a different scenario (after the lift), i.e. "The Trucks stop there", which is the BEFORE (many trucks stop there) for the CORE (you can take the trucks there) metonymy. Milton's reply (this goes back to the first 
scenario, i.e. asking for a lift) "I'd be much obliged" is the AFTER (I express my gratitude) for the CORE (you give me a lift).

So far the problem of requesting a lift is solved. But another problem shows up in Piper's next turn, i.e. “just so you know, I don't pick up hitchhikers.” Piper comes to the general view from Milton's specific case (asking for a lift, despite the fact that he helped fix the car) that Milton is a hitchhiker. This is surely the PART-WHOLE RELATING process, namely, relating the specific case to the general knowledge of hitchhiking she has already established. Then by saying that, she suggests that "I don't pick up hitchhikers and that now I give you a lift is because you helped fix the car". The above analysis shows how metonymy operates in solving the first problem of asking for a lift.

And now immediately after that comes the second problem: I really wouldn't want to give you a lift if it were not for your help of fixing the car. This indicates that Milton is in fact not welcome as a hitchhiker and that Piper rewards Milton more by giving him a lift than his help with the car really requires. It also suggests that Milton should thank her instead. This utterance by Piper perhaps also implies that she wants to make it clear to Milton that she is not the sort of girl who usually picks up hitchhikers, but this can still suggest that Milton should be grateful to her for the rare lift she gives. However, Milton makes good use of metonymy, related to the scenario of hitchhiking. A hitchhiker generally asks for a lift by sticking his thumb out. According to this scenario, a hitchhiker must stick his thumb out to let others know that he needs a lift. But it is clear that Milton did not do that. Thus Milton's reply "I didn't have my thumb out" refutes Piper's point that he is a hitchhiker, and removes her thought (You should thank me instead). Milton negates the RESULT by denying the CONDITION (BEFORE) component within the scenario framework of HITCHHIKING. It actually involves a CONDITION FOR RESULT metonymy in a negative sense. While Piper adopts a PART-WHOLE RELATING process to relate Milton's case to a hitchhiker and employs it to indicate that Milton should be grateful to her, Milton makes advantage of a PART-WHOLE RELATING process to deny the whole by denying the component part, i.e. the CONDITION or the BEFORE component ("I didn't have my thumb out") of the scenario. This 
interaction of metonymy between them finally successfully helps Milton solve the second problem and realize his desire.

In interviews, the structuring and developing function of metonymy is arguably more the responsibility of the interviewer. Correspondingly, the problem-solving function of metonymy is arguably more the responsibility of the interviewee. The following is an interview example in terms of problem-solving, focusing on the interviewee's reply. This interview is from WSC, which is between IM (a female broadcaster/journalist) and RR (a female, Minister of Finance) about New Zealand superannuation policy (WSC\#DGI072, see the CD attached).

Through the interview (fourteen turns in total), it will be found that the questions raised by the interviewer are very pointed, definitely posing problems to RR. As a reply to the first question (the first turn), RR first gives a definite negative answer and then gives the reasons for not looking at it. Of course it is acceptable to just say "No", but the problem seems not to be solved in RR's view. Thus she cites the reasons to provide more support and ground, suggesting that this ("the steep clawback for superannuitants earning more than eighty dollars a week from other investments") does not go to the budget nowadays. From the perspective of an event scenario, the question is about the HAPPENING, RR replies with the CONSEQUENCE (negative) and the REASON, disproving the HAPPENING and CONSEQUENCE by negating the REASON.

The second question is really acute, and it puts an adverse opinion on the Treasury by citing specific cases. RR concludes "that personal abuse deserves the contempt of my silence", her conclusive remarks are based on IM's specific case of Michael Laws. It is a SPECIFIC TO GENERAL (PART-WHOLE FORMING) process as well as the involvement of a RESULT FOR EVALUATION metonymy. That is, RR utters the RESULT to show her EVALUATION that Michael Laws' words are nonsense and deserve contempt. Then IM points out that this is not a specific case but a common opinion shared among people (the third turn). Following that, RR starts to talk about the government, because the Treasury is part of the government, and then extends the specific case to people. Thus she employs PART TO WHOLE, SPECIFIC TO GENERAL, and PART TO PART metonymies to temporarily solve the problem: From the Treasury to the Government, from the Michael Laws case to 
the public, from a public issue to personal abuse (which is also an EVALUATION FOR RESULT metonymy).

IM does not agree that there is any personal abuse in the public's questioning of the Treasury (the fourth turn). How to solve this problem? RR still adheres to the talk on the government in general, and the relation between the Treasury and the government in particular, and comes to the idea that the government is accountable for everything. So this reply is a specific aspect of RR's general idea in her previous turn (the government is accountable for everything), followed by an announcement that her government is different (it acts on its own instinct, not on advice). Therefore, an overall GENERAL TO SPECIFIC metonymy motivates her reply here. It also involves the metonymies GOVERNMENT ACTING ON ITS OWN INSTINCT FOR TRUSTWORTHY GOVERNMENT and GOVERNMENT ONLY ACTING ON ADVICE FOR TRUSTLESS GOVERNMENT. IM then points out that there is a great deal of dissent in this government (general) and four of them have crossed the floor (specific) (the fifth turn). IM's utterance here poses a QUESTION FOR NEGATIVE STATEMENT metonymy. The question of how many is metonymically presented to imply a negative situation, i.e. there seems to be very few on RR's side. In reply to IM, RR concludes that this is media hype (MEDIA HYPE FOR FALSE INFORMATION) and hype does not produce good government (HYPE FOR HINDRANCE TO GOVERNMENT), and cites specific cases. RR's reply also follows procedure of GENERAL TO SPECIFIC TO GENERAL.

In answer to IM's question as to why there is so much dissent within her own government on this issue (the sixth turn), overall RR still adopts the GENERAL TO SPECIFIC TO GENERAL process. She first gives a negative answer and then comes to a general idea (the government has secured support for its measures), then to specific cases (the backbench commitment, the cabinet commitment), followed by a general idea (it was the most democratically written budget in the country's history and is the sounder for it). The last sentence by RR also involves an EVALUATION FOR RESULT metonymy, implying this budget is so sound that the public should accept it without any argument (personal abuse). IM then asks a specific question, i.e. whether it concerns RR that backbenchers like Michael Laws are speaking out publicly (the seventh turn). RR's reply uses the Prime Minister's wording to express 
his opinion (PRIME MINISTER'S IDEA FOR MY IDEA). In order to show the opposite opinion to RR's (they haven't prevailed), IM starts to read the letters from those people who question the budget (from the eighth turn till the last). All these turns are motivated by the INDIVIDUAL CASES FOR THE WHOLE SITUATION metonymy, a subtype of SPECIFIC FOR GENERAL.

The first related question is whether the new joint income testing is fair (the eighth turn). RR's answer gives a positive answer first and then gives the general reason (taking family as a basis/unit for income testing), followed by interpreting the corresponding specific cases mentioned in IM's talk and then a general idea (we look at the reality that most of us have the happiness of a family base and pool our income as a family). Following that, IM goes to a special case in which people retain their separate incomes (general) and cites a specific case (the ninth turn), i.e. from GENERAL (a lot of people retain their separate incomes) to SPECIFIC (the case of Ruth Allen of Ponsonby). And RR's reply to the question concerning Ruth Allen of Ponsonby is first claiming that in the specific case (i.e. Ruth Allen's family) that family is still assessed as a family unit and that it is up to them to determine how to manage the family income internally, then stating the general procedure followed by the government (taking the family as a unit), followed by such specific case analyses as higher income family and lower income family. So it is clear that the progression of RR's reply is from SPECIFIC (the Ruth Allen case) to GENERAL (the government's procedure) and to SPECIFIC (the higher income family and lower income family cases).

IM's following turn (the tenth turn) consists of a general opinion drawn from the specific assessment (referring to the kind of logic which suggests to some people that they would be better off to divorce and blow all their savings) (SPECIFIC TO GENERAL), and then comes to specific cases (Hughes of Whangarei). All the above is about family, but this case brings marriage to the fore. RR's reply comes to the general from this case (this is the most frequently asked question) and talks about the general situation (de facto relationships are in the nature of marriage and are treated as the same). Of course, following RR's reply, IM naturally comes to the question (how are you going to establish that, i.e. how do you tell they are in the relationship of "marriage") (the eleventh turn). RR first replies by saying which department is 
responsible for making the judgement of de facto relationships, and then gives a general statement followed by specific cases (sisters or brothers) and then comes to the general announcement ("in our welfare system we have never made a distinction between the allowable earnings for a couple and the allowable earnings for an individual").

Following RR's turn, especially regarding the notion of “individual” in RR's turn, IM poses a special case (the twelfth turn), i.e. brothers and sisters as individuals sharing expenses in the same house, indicating that they should be treated as a family given what RR has talked about. RR replies with a general statement ("families are complex things") and then cites special cases like those that IM presented but comes to the general research result ("the studies that have been done by the department of social welfare show that single people tend to lead single existences"), followed by further talk about the special cases IM presented (brothers and sisters, though sharing the same house and some expenses, are treated as independent units with good reason). Thus RR employs the GENERAL TO SPECIFIC metonymy in her reply to solve the special problem posed by IM. Of course there are some other metonymies further involved, e.g. RESEARCH RESULT FOR REALITY metonymy when RR presents the result of the studies.

In her next question (the thirteenth turn), IM presents another specific case regarding superannuation (whether Frank Bailey of Hamilton should spend his superannuation from his firm so as to receive a larger fraction of the state pension). RR's reply gives a negative opinion with the reason, moving from Frank to those in Frank's position and to anybody whose superannuation is reduced. It develops from SPECIFIC TO GENERAL. But RR's reply also involves an ASSERTION FOR SUGGESTION metonymy, in which her opinion and the statement of reason function as a suggestion to people of what to do.

The above analysis illustrates that in this interview, the interviewee (RR), is often pressed by all kinds of pointed questions and problems from the interviewer (IM), and that RR's replies to the questions and solutions to the problems are overall 
modelled by GENERAL-SPECIFIC ${ }^{83}$ metonymy. She replies sometimes in the way of SPECIAL TO GENERAL, sometimes in GENEAL TO SPECIFIC, sometimes in GENERAL TO SPECIFIC TO GENERAL, sometimes in SPECIFIC TO GENERAL TO SPECIFIC, and so on. At the same time, RR's (and also IM's) utterances involve a variety of its subtypes and are embedded with some other metonymies, as analysed above. All these are procedures or solutions made after her decision-making process; or at least in her mind, these might be the best way to solve the problems on the spot. She might have done that without any deep thought of how the problems can be tactically tackled; however, the above analysis shows that her problem-solving in fact involves some patterns of the GENERAL-SPECIFIC metonymy and other metonymies are also underlying her replies.

\subsection{Summary}

Metonymy has long been regarded as a mechanism of semantic extension and development. This function is, most of the time, confined to the local interpretation of metonymically motivated words or expressions in the sentences. In fact, metonymy can also contribute to the whole dialogue scenario. This is taken as the meaning-making of metonymy. It is taken as a means to contribute to the dialogic discourse as a whole entity in terms of meaning. The meaning-making of metonymy can uncover and clarify what is otherwise missed out in the exclusive focus on the local sentences. The meaning-making of metonymy relates the local interpretation to the dialogic discourse scenario, making more meanings out of the utterance from the perspective of dialogic discourse.

In dialogic discourse, every utterance is the final choice made by the speaker after a decision-making process. Metonymy is involved not only in making meanings but also in the decision-making process. It helps the speaker make inferences and decide what to utter among alternatives before he presents his idea or opinion. The decisionmaking of metonymy rests on the inferences the speaker makes regarding what to say, it is usually related to what he hears from his counterpart. Therefore the decision-making of metonymy is interactive between the participants in the dialogue, and involves an event scenario or action scenario.

\footnotetext{
${ }^{83}$ But in the continual progression of utterance, it might be bettered termed as (SPECIFIC-) GENERAL-SPECIFIC (-GENERAL), that is, the metonymic pattern GENERAL-SPECIFIC.
} 
Decision-making is usually a part of a problem-solving process. Making a decision is to solve problems. Metonymy is also seen to operate in answering questions and solving problems in dialogic discourse. The problem-solving aspect of metonymy helps the speaker formulate and organize his reply and solution in an appropriate and efficient manner after he has made an inference and a decision. The problem-solving of metonymy is embodied in various patterns of a GENERAL-SPECIFIC model and other forms of metonymy in dialogic discourse. It does not only operate in verbal communication to solve internal problems invited by the dialogue, e.g. the above interview (WSC\#DGI072), but also operates to solve external actual problems posed by the reality, e.g. the Drive Angry instance of requesting a lift.

Meaning-making, decision-making, and problem-solving are different aspects of metonymy as a cognitive mechanism in dialogic communication; they are interrelated but have different perspectives in focus, e.g. meaning-making focuses on the meaning of specific utterances at the discourse level, decision-making on the selection process, and problem-solving on the resolution of issues; they contribute to the dialogic discourse as a whole entity in an integrative way. These functions of metonymy are presented from the conceptual perspective of dialogic discourse. Nevertheless, metonymy can serve different discourse functions, some of which have been involved in the above analysis, e.g. attitudinal (EFFECT FOR ATTITUDE), affective (RESULT FOR EVALUATION). Metonymy operates in conceptual perspectivizations, and its other functions at the dialogic discourse level such as euphemistic and ironical use are also worth detailed examination. 


\section{Chapter 10 Conclusion}

\subsection{Summary of the main points}

In traditional rhetoric metonymy is regarded as a replacement of words, especially nouns, focusing on the rhetorical effects of this noun substitution in the sentence. In historical semantics, metonymy is viewed as one of the mechanisms of meaning extension, focusing on the diachronic semantic change of words. In the structural approach to language, metonymy is considered as a syntagmatic relation or a modification relation. In the cognitive approach, metonymy is taken as a cognitive model, a cognitive process which underlies language structure and language use, motivating pragmatic inferences. The cognitive view of metonymy extends the research on metonymy from nouns to all word classes; from the semantic aspect to the syntactic aspect, to the pragmatic aspect; from words to phrases, to sentences, and to discourse; from the linguistic category to conceptual category, from intraword-class to inter-word-class.

Cognitive Linguistics typically defines metonymy as a cognitive process in which the source provides mental access to the target within the same domain or Idealized Cognitive Model, but the terminology adopted, e.g. domain, has not been satisfactorily clarified, though plenty of effort has been made. In Cognitive Linguistics, besides the cognitive domain approach, there are some other approaches, e.g. the cognitive mental access approach or reference point approach, the conceptual blending theory, and the contingent indexical approach. The cognitive mental access approach sees metonymy as a conceptual reference point phenomenon providing mental access to the target based on a conceptual contiguity relationship. But it seems that this approach cannot spell out the distinction between metonymy and metaphor in terms of working mechanism; besides, it pays more attention to the referential aspect of metonymy. The conceptual blending approach treats metonymy as a blending phenomenon involving several mental spaces. While this approach tries to interpret metonymy, it actually needs metonymy to play a curial role in the working operation, and it seems to fall into a circular reasoning. The contingent indexical approach takes metonymic relations as indexical, which unfortunately excludes those metonymies which are instead bases on iconic relation. 
The cognitive domain approach adopts mapping and domain in its view of metonymy, taking metonymy as an intra-domain mapping. However, it seems that mapping is in fact a substitution relation and more suitable for metaphor, and domain involves more problems such as fuzzy boundaries, challenging domain decision, and changeable perspectivization.

Even so, the cognitive domain approach is the most widely accepted and employed in metonymy research. According to this view, metonymy bears some features such as the fact that it is a cognitive process, it takes place within a whole called domain/ICM, with PART-WHOLE as the prototypical relation, and it is ubiquitous in language, and more basic than metaphor. Nevertheless, as summarized in Chapter 3 , the prototypical view of metonymy in Cognitive Linguistics inevitably invites some questions, e.g. if metonymy is a cognitive process, what underpins its operation? Does metonymy operate in the forming of the WHOLE in addition to its operation within the whole? Does metonymy operate in cognitive activities without language involvement or with language influence least felt and if so, how does it work?

These questions can be satisfactorily answered when metonymy is taken as an innate cognitive mechanism involving PART-WHOLE FORMING and PART-WHOLE RELATING processes in the light of Embodied Cognition research. Cognitive science suggests that a body schema has been well formed through the prenatal bodily movement and it shapes the mind. It is likely that the body schema imposes a cognitive frame (WHOLE). But this WHOLE is only a prototype and needs to be configured, to be enriched, clarified, and developed. It is in this sense that PARTWHOLE FROMING process works. The PART-WHOLE FORMING process operates in the establishment of a conceptual whole pattern such as categorization, conceptualization, action scenario, event scenario. Once this whole pattern is formed through embodied experience, the PART-WHOLE RELATING process is likely to operate within the WHOLE pattern, relating a PART to another PART and relating a PART with the WHOLE or relating the WHOLE with a PART, e.g. in category and concept development, in inductive inference, and in memory function. Moreover, research in cognitive science indicates that metonymy operates in infancy and in subjects with brain damage when those essential cognitive activities such as 
conceptualization, categorization, inference, and memory are looked at, and these are cases in which metonymy operates beyond language involvement. Within the framework of Embodied Cognition, the usually regarded inter-domain relation between space and time can thus be primarily viewed as a PART-PART RELATING, for embodied cognitive activities are concerned with both time and space; these two so-called domains are therefore inseparable aspects of an embodied experience. Cognitive science research suggests that metonymy is more elementary in the mind than metaphor and metaphor is conceptually grounded on metonymic operation.

Metonymy in the mind definitely shows up in language and language use. The research on metonymy in Cognitive Linguistics generally takes it as a motivation underlying language in terms of meaning and structure. Nevertheless, there are some areas left uncovered in the metonymy research, e.g. grammaticalization and meaning prototype. Grammaticalization is basically regarded as a syntactic change and development that proceeds from concrete to abstract, but this research shows that grammaticalization is usually supported by metonymy. Rather than the widely-held idea of concrete- to- abstract process, grammaticalization proceeds from the general/global to the specific when viewed from the conceptual perspective. In Cognitive Linguistics, meanings, especially word meanings, are taken as accommodated around a prototype, i.e. a prototypical meaning. This view of prototypical meaning is essentially a static view of meaning and meaning change, because it sees the prototypical meaning as the core or kernel that all other meanings are centred around. The dynamism of prototype is proposed in the literature, but it has not been thoroughly addressed. It might be said that every polysemous word has a meaning prototype at a certain stage. The meaning prototype is not fixed once and for all but is developing and changing with discourse situation evolvement. The dynamic of meaning prototype is generally advancing from the general/global to the specific in terms of meaning, underpinned by metonymy as a natural cognitive mechanism. In cognition the general/global whole is formed well before the whole exerts influence. As a special case of common language use, the conceptual analysis of football nicknames also illustrates the point that metonymy underlies the nickname forming as an essential operation, and that metonymy brings in other cognitive and communicative functions through its underlying operation. 
Metonymy is studied in language and is also touched on in discourse in Cognitive Linguistics, how metonymy operates in interactive communication, especially in dialogic discourse, and how it contributes to the dialogic discourse as a coherent entity is, nevertheless, not explored in full. The research on metonymy in dialogic discourse has to, at first, have a clear idea of what metonymy is and how it works in both the mind and language, as discussed in the first two parts of this thesis. Metonymy operates in the mind as an inherent cognitive mechanism; it motivates language structure and language use, and underlies language development. It also functions to make contributions to active communication. In dialogic discourse metonymy operates in at least four aspects from both the conceptual and the discourse perspectives: Structuring and developing, which helps format the dialogue and makes it a coherent entity; meaning-making, which relates the local utterance to the discourse level and contributes to the whole dialogue as a discourse entity; decision-making, which underlies the speaker's utterance decisions from alternatives in response to what he hears; and problem-solving, which helps the speaker, especially the interviewee in this research, organize his thoughts and present his utterance in an effective and persuasive way. The functions metonymy serves and the contributions it makes rest mainly on the various forms of its GENERALSPECIFIC conceptual pattern embedded in the dialogic discourse, strewn with speech act and some other metonymies.

\subsection{Implications of the research}

Metonymy as a natural cognitive mechanism motivates human thinking, reasoning, and underlies language and communication. Metonymy is in fact the nature of embodied cognition; PART-WHOLE FORMING and PART-WHOLE/PART RELATING characterise the basic cognitive processes of Embodied Cognition such as conceptualization, categorization, memory, and inference. These processes of metonymy often interact and operate unconsciously in the mind, in language, and in communication. The current cognitive research on metonymy falls into the second PART-WHOLE/PART RELATING process, the presentation of the PART-WHOLE FORMING process complements it in terms of theory and operation, making metonymy a fully cognitive mechanism with great descriptive and interpretive power for both language and communication. The WHOLE configured through PART- 
WHOLE FORMING is rather general and global, but it is the precondition of the PART-WHOLE/PART RELATING process, and guides inference, conceptual development, and categorical development. This general/global/abstract- to- specific movement of metonymy in conceptual activities is significant in offering reasonable interpretations for some language and communicative phenomena, as shown in PART TWO and PART THREE. Besides, it poses a challenge to the commonly-held view of concrete- to- abstract conceptual development. Concept is grounded on perceptual experiences and sensorimotor activities, but this is only a concrete percept and is not a concept. Concept is different from percept in that concept is general and global at the first place and comes to specific in cognitive development and language acquisition, as illustrated by the research experiments in cognitive psychology and developmental psychology. This is why some other word has to be added before a noun (concept) in order to have a specific concept, e.g. car, racing car, red car in English, and che1 (car), pao3che1 (racing car), hong2se4deche1 (red car) in Chinese. This point of metonymy is crucial and beneficial to current cognitive perspectives of language and language use, of language acquisition and language teaching.

The presentation of the PART-WHOLE FORMING process in metonymy also makes it clear that metonymy is so essential to Embodied Cognition that metaphor is only secondary and grounded on metonymy in cognition. It also provides empirical support and evidence for the claim that metaphor is essentially based on metonymy. In addition, the general- to- specific view of grammaticalization in light of metonymy as an inherent mechanism involving PART-WHOLE FORMING and PART-WHOLE/PART RELATING posits a whole new perspective on grammaticalization, providing a fruitful analysis method to solve the conflict (i.e. the concrete- to- abstract unidirectionality and some general-to-specific grammaticalization cases, e.g. in auxiliation) existing in current grammaticalization research. Football nicknaming is good evidence to show that the two processes of metonymy interact in conceptualization and categorization; one the one hand, the PART-WHOLE FORMING process underlies the forming of football nicknames, and on the other hand, the PART-WHOLE/PART RELATING process underpins inferences and brings in specific communicative and cognitive functions. This also suggests that metonymy is not merely a cognitive process of relating PART and WHOLE, as held in current cognitive research on metonymy, but a natural cognitive 
mechanism involving both PART-WHOLE FORMING and PART-WHOLE/PART RELATING. This embodied view of metonymy is consistent with current cognitive science research and may be employed to fruitfully describe and interpret various communicative phenomena.

The analysis of metonymy in interaction, especially in dialogic discourse, complements current research on metonymy in discourse, which is mainly conducted on narrative discourse. Even where it is done on dialogue, the weight is mostly placed on speech act metonymies, with some on the function of making inferences. The conceptual analysis of metonymy in dialogic discourse in terms of structuring and developing, meaning-making, decision-making, and problem-solving is done probably first in this thesis. This approach provides a novel perspective on metonymy research, raising the contributions of metonymy to dialogic discourse as a coherent entity. This is different from the prevailing research on metonymy which places its effort mainly on the local meanings metonymy makes and the roles metonymy plays in local talk turns in terms of pragmatic inferences and illocutionary function. The analysis of silenced dialogue also indicates that metonymy not only operates in language-involved conceptual activities but also functions in situations beyond language, suggesting the ubiquity of metonymy as an innate cognitive mechanism in the mind.

All in all, the embodied view of metonymy advocates an integrated account of both the analysis of metonymy and the analysis of embodied cognitive activities, which is in line with the gist of Cognitive Linguistics (e.g. no distinction between semantics and pragmatics in principle, the central role of encyclopaedic knowledge). The view of metonymy as an inherent cognitive mechanism involving PART-WHOLE FORMING and PART-WHOLE/PART RELATING not only complements current research on metonymy in theory, telling why metonymy is ubiquitous in language and powerful in language use from the perspective of embodied cognition, but also provides innovative ideas and perspectives for research on metonymy application in language and extra-linguistic areas, as instanced in PART TWO and PART THREE, offering implications for further research in language and communication. 


\subsection{Areas of future research}

Language use is various and involves infinite aspects. The view of metonymy held in this research (i.e. metonymy as an innate cognitive mechanism involving both PART-WHOLE FORMING and PART-WHOLE RELATING) has many implications, and the research of metonymy in both language-involved activities and beyond-language activities can break new ground, what has been looked at in this thesis is only waves of the ocean. Even in the waves that are accounted for in this research more work still needs to be done.

For example, in the METONYNMY IN THE MIND part, it is argued that time and space are inseparable aspects of any embodied cognitive activity, and time is thus spatially conceptualized through metonymy, at least primarily, and this point seems to be neglected in current Cognitive Linguistics and cognitive science. According to the theory of metonymy held in this research, it is possible to have the hypothesis that time as an aspect of any cognitive activity might be otherwise conceptualized as well. Given that temporal concepts are spatially configured, how exactly they are spatially represented might vary from one language community or tribe to another due to specific embodied experience in different environment. The Amondawa case can be taken as an instance, more instances are needed for further examination, i.e. more endangered languages or minor languages should be taken into account for further evidence.

Another aspect in this part is the relation between metonymy and metaphor. It is argued that metaphor is conceptually grounded on metonymy with some analysis of aphasia, but more evidence is needed for the relation between metonymy and metaphor in subjects with cognitive impairments, e.g. aphasia, dyslexia, and other language disorders. Besides, while the research on metaphor in Cognitive Linguistics leads to research into the neural networking in the brain, it is also worthwhile to do research on metonymy and the distinction between metonymy and metaphor, in terms of neural circuits or patterns, given that they are cognitive mechanisms, and to examine what relation exists between the neural patterns if they are found to be distinguishably active in metonymy and metaphor. It would be interesting to do such research since Embodied Cognition theory advocates multimodal cognition (i.e. 
cognition is grounded on perceptual and sensorimotor experience that arises across the sensory modalities, proprioception, and introspection in the form of neural representations, see Barsalou (1999b). Embodied Cognition is not considered as amodal (i.e. the idea that cognitive representations are inherently non-perceptual) (Barsalou, 1999a; 1999b, 2003) any more.

In the METONYMY IN LANGUAGE part, only typical aspects of language and language use, e.g. grammaticalization, meaning prototype, football nicknames, are considered. As said at the beginning of this section, language and language use involves various aspects in terms of communication. Besides what has been researched in the literature of metonymy and what has been considered in this research, there are many other aspects that also need to take a metonymic perspective in research to further metonymy study in terms of application, for example:

1) In the grammaticalization chapter, only be going to is considered; more effort is in need to examine whether and how metonymy functions in the grammaticalization process of other auxiliary words such as can and must in English and other similar functional words in other languages.

2) How does metonymy function in language acquisition and language development (especially in second language or foreign language learning)?

3) Is there any significance of metonymy in language teaching (especially in second language or foreign language teaching)?

4) Does metonymy contribute to interpretation and translation? And if so, how does it operate and to what degree?

5) Some football clubs share the same nickname, besides the shared underlying cognitive mechanism, does there exist any other conceptual difference in this case?

The METONYMY IN COMMUNICATION part examines how metonymy operates in and contributes to verbal interactions, and particular consideration is given to dialogic discourse, especially interviews, taken from corpora. But interviews are comparatively heavily pre-formed in terms of question and answer. Some other forms should be given more space in the developing analysis of metonymy in dialogue. Although other dialogues are also taken into account, such as movie dialogues and silenced dialogues, it is inevitable that some are left unexplored. Thus 
more research needs to be done in other forms of dialogic discourse such as private conversations (both direct and by distance), interactive meetings and discussions, and business transactions. Another aspect with the corpora is that the corpora employed in this research are particularly goal-directed, e.g. ICE-NZ is mainly done for consideration of New Zealand English, especially for New Zealand English pronunciation, and the data recorded are not really complete dialogues or conversations, as shown in the analysis. Therefore the data used here is unavoidably confined and complete dialogues are needed for the analysis of metonymy in interactive communication.

There are also some other aspects to be taken into consideration in future research of metonymy in interaction. Most of the examples in this part are language-internally focused, i.e. they are focused on language use for internal structure and language related problems. This part also touched on metonymy in solving external realistic problems, e.g. metonymy underlies the Drive Angry case to solve the lift-taking problem and the thanks-giving problem, but data of this kind deserve more attention in future research on the problem-solving aspect of metonymy.

Apart from the above, the function inventory of metonymy in dialogic discourse should probably not be restricted to structuring and developing, meaning-making, decision-making, and problem-solving. Other possible functions of metonymy such as its euphemistic, ironical, attitudinal, and affective uses also deserve a space in the research. Even in the analysis of those functions, e.g. the structuring and developing function of metonymy, some component is not well attended to; for instance, how the interviewee contributes to the development of the dialogic discourse in terms of metonymic function is not really touched on due to the emphasis is placed on the interviewer. Necessity is thus felt to study the discourse functions of metonymy in long texts and natural dialogic discourse in a systematic way.

In addition, to follow the neurological trend in the research on metaphor and language within the framework of cognitive science (e.g. Dodge \& Lakoff, 2005; Feldman \& Narayanan, 2004; Hubbard et al., 2005; Hubbard \& Teuscher, 2010; Knapp \& Corina, 2010; Lacey, Stilla, \& Sathian, in press; Lakoff, 2009; Mashal et al., 2007; Willems \& Hagoort, 2007), the study of metonymy might better to be taken from the neurological perspective in the future research as well. It would be 
sounder to bring cross-disciplinary effort together to neurologically testify metonymy as a natural cognitive mechanism with the methodology used in neuroscience and cognitive science such as Electroencephalography (EEG), Eventrelated potentials (ERPs), Functional magnetic resonance imaging (fMRI), and to examine how and to what extent metonymy operates in nonverbal (especially preverbal) cognitive activities with the help of eye tracking, reaction time experiments.

These are all worthwhile aspects of future research on metonymy from the conceptual perspective. 


\title{
Appendix 1: Examples of the Metaphoric Extension of "110"
}

\author{
(All from People Website www.people.com.cn)
}

IP 110 (IP 110)

帮扶 bang1fu2 110 (Assistance and Support 110)

暴政 bao4zheng4 110 (Maladministration 110) (a reportage title)

本科教学质量 ben3ke1jiao4xue2 110 (Undergraduate Teaching Quality 110)

边境 bian1jing4 110 (Border 110 (armed force on the border))

草原 cao3yuan2 110 (Grassland 110)

城管 cheng2guan3 110 (Municipal Administration 110)

城建 cheng2jian4 110 (Urban Construction 110)

储蓄 chu3xu4 110 (Deposit 110)

畜牧 xu4mu4 110 (Animal Husbandry 110)

春运 chun1yun4 110 (Spring Festival Transportation 110)

打炒 da3chao3 110 (Anti-speculation 110)

党员 dang3yuan2 110 (Party members 110)

的士 di2shi4 110 (Taxi 110) (the taxi drivers act as clue and help provider to the police)

地下 di4xia4 110 (Underground 110)

电力 dian4li4 110 (Power 110)

电信 dian4xin4 110 (Telecom 110)

都市 du1 shi4 110 (Metropolis 110)

短信 duan3xin4 110 (Texting 110)

反伪 fan3wei3 110 (Anti-fake 110)

房产 fang2chan3 110 (House Property 110 )

港口 gang3kou3 110 (Harbour 110 (police at the harbour))

高考 gao1kao3 110 (University Entrance Examination 110)

工厂 gong1chang3 110 (Factory 110)

公务 gong1wu4 110 (Public Service 110)

供水 gong4shui3/自来水 zi4lai2shui3 110 (Water 110)

广告 guang3gao4 110 (Ads 110)

规划 gui1 hua4 110 (Urban Planning 110)

海关 hai3guan1 110 (Customs 110)

海路 hai3lu4 110 (Sea and Land 110 (police on both land and sea))

海上 hai3shang4 110 (Sea 110 (police on sea)) 
行政 xing2zheng4 110 (Government Administration 110)

和谐 he2xie2 110 (Harmoniousness 110)

环保 huan2bao3 110 (Environment Protection 110)

火车 huo3che1 110 (Train 110)

机车 ji1che1 110 (Locomotive 110)

机关效能 ji1guan1xiao4neng2 110 (Institution Efficiency 110)

加油计量 jia1you2ji4liang4 110 (Fueling Measure 110)

经济 jing1ji4 110 (Economy 110)

纠风 jiu1feng1 110 小分队 (Rectifying 110)

炕头 kang4tou2 110 (Kang-bed 110)

科技 ke1ji4 110 (Science and Technology 110)

科普 ke1pu3 110 (Science Popularization 110)

口腔 kou3qiang1 110 (Stomatology 110)

劳务 lao2wu4 110 (Labour Service 110)

粮食 niang2shi2 110 (Grain 110)

论文 lun4wen2 110 (Paper 110)

旅游 lv3youl 110 (Tourism 110)

麻将 ma2jiang4 110 (Mahjong 110)

马背 ma3bei4 110 (Horse 110 (horse police))

煤气 mei2qi4 110 (Gas 110)

民间 min2jian1 110 (Non-government 110)

民生 min2sheng1 110 (People's Livelihood 110)

民声 min2sheng1 110 (Civilian Voice 110)

民事 min2shi4 110 (Civil Affairs 110)

民政 min2zheng4 110 (Civil Administration 110)

农技 nong2ji4 110 (Agro technical 110)

农事 nong2shi4 110 (Farm Work 110)

扑克 pu3ke4 110 (Poke 110)

气象 qi4xiang4 110 (Weather 110)

汽配 qi4pei4 110 (Automobile Fitting 110)

人大 ren2da4 110 (People's Congress 110)

人大换届选举 ren2da4huan4jie4xuan3ju3 110 (Election 110 of People's Representatives)

人大监督 ren2da4jian1du1 110 (People's Representative Intendance 110)

森林 sen1lin2 110 (Forestry 110 (police for the forestry)) 
杀毒 sha1du2 110 (Virus killing 110)

沙漠 sha1mo4 110 (Desert 110 (police patrol on desert))

社区 she4qu1 110 (Community 110)

生存智慧 sheng1cun2zhi4hui4 110 (Survival Wisdom 110)

食品安全 shi2pin3an1quan2 110 (Food Safe 110)

书记 shu1ji4 110 (Party Secretary 110)

数学 shu4xue2 110 (Maths 110)

水上 shui3shang4 110 (River 110 (police patrol on river))

诉讼 su4song4 110 (Legal Action 110)

台风 tai2feng1 110 (Typhoon 110)

通关 tong1guan1 110 (Customs Declaration 110)

铜都盐业 tong2du1 yan2ye4 110 (Copper Urban Salt 110)

玩家 wan2jia1 110 (Player 110)

网络 wang3luo4 110 (Internet 110)

网上 wang3shang4 110 (Internet police 110)

为民服务 wei4min2fu2wu4 110 (Serving-the-People 110)

文化强国 wen2hua4qiang2guo1 110 (Cultural Power 110)

物管 wu4guan3 110 (Property Management 110)

心理 xin1li3 110 (Psychotherapy 110)

新闻 xin1wen2 110 (News 110)

养老 yang3lao3 110 (Support 110 for the Old-aged)

义务 yi4wu4 110 (Volunteering 110)

娱乐 yu2le4 110 (Entertainment 110)

远 (程) 教 (育) yuan3cheng2jiao4yu4 110 (Distance Learning 110)

证券 zheng4quan4 110 (Stock Market 110)

志愿者 zhi4yuan4zhe3 110 (Volunteers 110) 


\section{Appendix 2: The Nicknames of Football Club Teams (English Premier League 2009-2010)}

\begin{tabular}{|c|c|c|c|}
\hline No. & Home Club & Nickname & Reason \\
\hline 1. & Crystal Palace & Eagles, The & $\begin{array}{l}\text { After the eagle featured on the club } \\
\text { badge (crest) since the Seventies (1973). }\end{array}$ \\
\hline 2. & Birmingham City & Blues, The & Colour of home football kit \\
\hline 3. & Burnley & Clarets, The & Colour of home strip. \\
\hline 4. & Chelsea & Blues, The & Colour of home football kit. \\
\hline 5. & Coventry City & Sky Blues, The & Colour of home strip \\
\hline 6. & Liverpool & Reds, The & Colour of home kit. \\
\hline 7. & Manchester City & Blues, The & Refers to light blue home strip \\
\hline 8. & $\begin{array}{l}\text { Nottingham } \\
\text { Forest }\end{array}$ & Reds, The & Colour of home kit \\
\hline 9. & Portsmouth & Blues, The & Colour of home kit \\
\hline 10. & $\begin{array}{l}\text { Preston North } \\
\text { End }\end{array}$ & $\begin{array}{l}\text { Lilywhites, } \\
\text { The }\end{array}$ & $\begin{array}{l}\text { A reference to home strip: White shirts } \\
\text { and blue shorts }\end{array}$ \\
\hline 11. & \begin{tabular}{|l} 
Tottenham \\
Hotspur
\end{tabular} & Lilywhites, & $\begin{array}{l}\text { Colour of home strip: White shirts and } \\
\text { blue shorts }\end{array}$ \\
\hline 12. & $\begin{array}{l}\text { Manchester } \\
\text { United }\end{array}$ & $\begin{array}{l}\text { Red Devils, } \\
\text { The }\end{array}$ & $\begin{array}{l}\text { The nickname originally belonged to } \\
\text { nearby rugby league team Salford } \\
\text { (Salford City Reds, a professional rugby } \\
\text { league club based in Salford in Greater } \\
\text { Manchester, England), and was } \\
\text { "borrowed" by Man United fans. The } \\
\text { name the Red Devils comes from an } \\
\text { early tour to France, where the French } \\
\text { press labeled them as playing like devils, } \\
\text { thus the name Les Diables Rouges, } \\
\text { which translates into English as the Red } \\
\text { Devils }\end{array}$ \\
\hline 13. & Aston Villa & Villans, The & Derived from "Villa" \\
\hline 14. & $\begin{array}{l}\text { Dagenham \& } \\
\text { Redbridge }\end{array}$ & Daggers, The & Derived from the club's name. \\
\hline 15. & Manchester City & Citizens, The & Derived from 'City' \\
\hline 16. & $\begin{array}{l}\text { Milton Keynes } \\
\text { Dons }\end{array}$ & Dons, The & $\begin{array}{l}\text { Derived from Wimbledon, a reference to } \\
\text { the club's origins as Wimbledon F.C. }\end{array}$ \\
\hline 17. & Port Vale & Valiants, The & Derived from Vale \\
\hline 18. & Bradford City & Bantams, The & $\begin{array}{l}\text { A reference to the claret and amber } \\
\text { colours of the club's strip }\end{array}$ \\
\hline 19. & Bristol City & Robins, The & $\begin{array}{l}\text { Red home kit similar in colour to a } \\
\text { robin's chest }\end{array}$ \\
\hline 20. & $\begin{array}{l}\text { Cheltenham } \\
\text { Town }\end{array}$ & Robins, The & $\begin{array}{l}\text { Red home kit similar in colour to a } \\
\text { robin's chest }\end{array}$ \\
\hline
\end{tabular}




\begin{tabular}{|c|c|c|c|}
\hline 21. & Hull City & Tigers, The & $\begin{array}{l}\text { Colour of the amber and black home } \\
\text { strip, They traditionally play in black and } \\
\text { amber, often with a striped shirt design }\end{array}$ \\
\hline 22. & Newcastle United & Magpies, The & $\begin{array}{l}\text { Based on black and white colours of the } \\
\text { home strip Magpies refers to the black } \\
\text { and white colours of the club, similar to } \\
\text { the Magpie bird }\end{array}$ \\
\hline 23. & Notts County & Magpies, The & $\begin{array}{l}\text { Based on black and white colour of } \\
\text { home strip }\end{array}$ \\
\hline 24. & Swindon Town & Robins, The & $\begin{array}{l}\text { Red home kit similar in colour to a } \\
\text { robin's chest }\end{array}$ \\
\hline 25. & Watford & Hornets, The & $\begin{array}{l}\text { Refers to the yellow and black home } \\
\text { strip }\end{array}$ \\
\hline 26. & $\begin{array}{l}\text { Colchester } \\
\text { United }\end{array}$ & U's, The & Initial of name (United). \\
\hline 27. & Leyton Orient & O's, The & Initial of name (Orient) \\
\hline 28. & $\begin{array}{l}\text { Queens Park } \\
\text { Rangers } \\
\end{array}$ & R's, The & Final initial of club abbreviation, QPR \\
\hline 29. & Barnsley & Tykes, The & Colloquial name for a Yorkshireman. \\
\hline 30. & $\begin{array}{l}\text { Bolton } \\
\text { Wanderers }\end{array}$ & Trotters, The & $\begin{array}{l}\text { In the } 19 \text { th century, one of their pitches } \\
\text { was adjacent to a piggery. And } \\
\text { clearances often end up in there, players } \\
\text { had to trot through the pigpens to } \\
\text { retrieve the balls }\end{array}$ \\
\hline 31. & Bristol Rovers & Pirates, The & Reference to Bristol's maritime heritage. \\
\hline 32. & Bury & Shakers, The & $\begin{array}{l}\text { Club's first chairman, JT Ingham, } \\
\text { commented before a game with } \\
\text { Blackburn, "We will shake them. In fact, } \\
\text { we are the Shakers." }\end{array}$ \\
\hline 33. & Cardiff City & Bluebirds, The & $\begin{array}{l}\text { Based on colour of home strip. From } \\
\text { Cardiff City mascot Bartley the Bluebird }\end{array}$ \\
\hline 34. & Darlington & Quakers, The & $\begin{array}{l}\text { A reference to the religious movement } \\
\text { (religious society of friends, called } \\
\text { quakers) which had a historic influence } \\
\text { on the town. }\end{array}$ \\
\hline 35. & Derby County & Rams, The & $\begin{array}{l}\text { A ram features as an emblem of both the } \\
\text { city and county, and also in the club's } \\
\text { badge. Folksong "The Derby Ram" }\end{array}$ \\
\hline 36. & Exeter City & Grecians, The & $\begin{array}{l}\text { Because of the club's association with St } \\
\text { Sidwell's Parish - people living in the } \\
\text { parish have been known as Grecians or } \\
\text { Greeks for many centuries. For centuries } \\
\text { the nickname for people from } \\
\text { St.Sidwell's Parish of Exeter where the } \\
\text { Ground is located }\end{array}$ \\
\hline 37. & Grimsby Town & Mariners, The & $\begin{array}{l}\text { Reference to Grimsby's maritime } \\
\text { heritage - It was once the largest fishing }\end{array}$ \\
\hline
\end{tabular}




\begin{tabular}{|c|c|c|c|}
\hline & & & port in the world. \\
\hline 38. & Lincoln City & Imps, The & $\begin{array}{l}\text { After the "Lincoln Imp" which, } \\
\text { according to legend, plagued Lincoln } \\
\text { Cathedral during the 14th century. } \\
\text { Legend of the imp statue in Lincoln } \\
\text { Cathedral }\end{array}$ \\
\hline 39. & Millwall & Lions, The & $\begin{array}{l}\text { Adopted the nickname after being } \\
\text { referred to as lions, due to their FA Cup } \\
\text { giant-killing exploits in } 1900 \text {. feature on } \\
\text { badge }\end{array}$ \\
\hline 40. & Newcastle United & Toon, The & $\begin{array}{l}\text { Originates from the Geordie } \\
\text { pronunciation of 'Town'. }\end{array}$ \\
\hline 41. & $\begin{array}{l}\text { Peterborough } \\
\text { United }\end{array}$ & Posh, The & $\begin{array}{l}\text { Reportedly derived from former manager } \\
\text { Pat Tirrel's comment that he was looking } \\
\text { for, "Posh players for a posh team". }\end{array}$ \\
\hline 42. & Plymouth Argyle & Pilgrims, The & $\begin{array}{l}\text { Plymouth was the departure point of the } \\
\text { Mayflower in } 1620 \text {, carrying the } \\
\text { pilgrims to the New World. }\end{array}$ \\
\hline 43. & Portsmouth & Pompey, & The nickname for the city of Portsmouth. \\
\hline 44. & Southampton & Saints, The & $\begin{array}{l}\text { The club was originally known as } \\
\text { Southampton St. Mary's. }\end{array}$ \\
\hline 45. & $\begin{array}{l}\text { West Bromwich } \\
\text { Albion }\end{array}$ & Baggies, The & $\begin{array}{l}\text { The club historian suggests that it } \\
\text { derives from "bagmen" who carried the } \\
\text { match-day takings from the turnstiles to } \\
\text { the cash office on the halfway line. }\end{array}$ \\
\hline 46. & $\begin{array}{l}\text { West Ham } \\
\text { United }\end{array}$ & Hammers, The & $\begin{array}{l}\text { The club's crest features crossed } \\
\text { metalworking hammers, referring to their } \\
\text { origins as Thames Ironworks. }\end{array}$ \\
\hline 47. & $\begin{array}{l}\text { West Ham } \\
\text { United }\end{array}$ & Irons, The & $\begin{array}{l}\text { The club was originally called Thames } \\
\text { Ironworks. }\end{array}$ \\
\hline 48. & Arsenal & Gunners, The & $\begin{array}{l}\text { The club were founded by workers from } \\
\text { the Woolwich armaments factory. }\end{array}$ \\
\hline 49. & Burton Albion & Brewers, The & Local link to brewing industry \\
\hline 50. & Everton & Toffees, The & $\begin{array}{l}\text { After a local shop which sold sweets, } \\
\text { including the "Everton Mint", on match } \\
\text { days. }\end{array}$ \\
\hline 51. & Ipswich Town & $\begin{array}{l}\text { Tractor Boys, } \\
\text { The }\end{array}$ & $\begin{array}{l}\text { Originally a derogatory chant by rival } \\
\text { fans referring to Ipswich's agricultural } \\
\text { heritage, the nickname was adopted by } \\
\text { Ipswich fans. }\end{array}$ \\
\hline 52. & $\begin{array}{l}\text { Macclesfield } \\
\text { Town }\end{array}$ & Silkmen, The & $\begin{array}{l}\text { Reference to the town's silk industry - } \\
\text { Macclesfield was once the world's } \\
\text { biggest producer of finished silk. }\end{array}$ \\
\hline 53. & $\begin{array}{l}\text { Northampton } \\
\text { Town }\end{array}$ & Cobblers, The & $\begin{array}{l}\text { Northampton was once a major centre of } \\
\text { the shoe making industry. }\end{array}$ \\
\hline 54. & $\begin{array}{l}\text { Scunthorpe } \\
\text { United }\end{array}$ & Iron, The & $\begin{array}{l}\text { Scunthorpe is the UK's largest steel } \\
\text { processing centre. }\end{array}$ \\
\hline
\end{tabular}




\begin{tabular}{|c|c|c|c|}
\hline 55. & Sheffield United & Blades, The & $\begin{array}{l}\text { Sheffield has been a major centre for } \\
\text { steel production and cutlery manufacture } \\
\text { since the } 18 \text { th century. }\end{array}$ \\
\hline 56. & Stockport County & Hatters, The & $\begin{array}{l}\text { Stockport was a major centre of the } \\
\text { hatting industry. }\end{array}$ \\
\hline 57. & Stoke City & Potters, The & $\begin{array}{l}\text { Stoke is a world renowned centre of the } \\
\text { pottery industry }\end{array}$ \\
\hline 58. & Walsall & Saddlers, The & $\begin{array}{l}\text { The town was a centre of the saddlery } \\
\text { industry from the Industrial Revolution } \\
\text { onwards. }\end{array}$ \\
\hline 59. & $\begin{array}{l}\text { Wycombe } \\
\text { Wanderers }\end{array}$ & Chariboys, The & $\begin{array}{l}\text { High Wycombe has been a centre of the } \\
\text { furniture production industry since the } \\
\text { 19th century. }\end{array}$ \\
\hline 60. & Yeovil Town & Glovers, The & $\begin{array}{l}\text { The glove making industry during the } \\
1800 \text { 's. }\end{array}$ \\
\hline 61. & Hereford United & Bulls, The & After the local breed of cattle \\
\hline 62. & $\begin{array}{l}\text { Huddersfield } \\
\text { Town }\end{array}$ & Terriers, The & $\begin{array}{l}\text { Named after the Yorkshire Terrier breed } \\
\text { of dog }\end{array}$ \\
\hline 63. & Leicester City & Foxes, The & $\begin{array}{l}\text { Reference to Leicestershire's hunting } \\
\text { tradition. Outline of Leicestershire on a } \\
\text { map resembles a fox's head }\end{array}$ \\
\hline 64. & Norwich City & Canaries, The & $\begin{array}{l}\text { Rearing canaries was a popular pastime } \\
\text { in the area during the early } 20 \text { th century. } \\
\text { The club adopted it's yellow strip } \\
\text { because of the nickname. }\end{array}$ \\
\hline 65. & Morecambe & Shrimps, The & $\begin{array}{l}\text { Morecambe Bay is a well known } \\
\text { shrimping area. }\end{array}$ \\
\hline 66. & Southend United & Shrimpers, The & $\begin{array}{l}\text { Reference to the local shrimping } \\
\text { industry. }\end{array}$ \\
\hline 67. & Charlton Athletic & Addicks, The & $\begin{array}{l}\text { Corruption of the word 'haddocks', } \\
\text { named after a local fishmonger. }\end{array}$ \\
\hline 68. & Barnet & Bees, The & $\begin{array}{l}\text { Based on the striped home kit. reflected } \\
\text { in their amber and black shirts, is likely } \\
\text { to have come from the location of } \\
\text { apiaries close to the Underhill ground in } \\
\text { the early years of the 20th century. }\end{array}$ \\
\hline 69. & Blackpool & Seasiders, The & Blackpool is a famous seaside resort. \\
\hline 70. & Bournemouth & Cherries, The & $\begin{array}{l}\text { Reference to a cherry plantation near the } \\
\text { ground. Former owner owned Cherry } \\
\text { Tree Plantation near the Ground }\end{array}$ \\
\hline 71. & $\begin{array}{l}\text { Brighton \& Hove } \\
\text { Albion }\end{array}$ & Seagulls, The & $\begin{array}{l}\text { Brighton is a seaside resort; therefore the } \\
\text { nickname refers to a sea bird. Also a } \\
\text { response to the similar sounding } \\
\text { nickname Eagles of their arch rivals } \\
\text { Crystal Palace }\end{array}$ \\
\hline 72. & Carlisle United & $\begin{array}{l}\text { Cumbrians, } \\
\text { The }\end{array}$ & $\begin{array}{l}\text { From Cumbria, the county the club hails } \\
\text { from. }\end{array}$ \\
\hline
\end{tabular}




\begin{tabular}{|c|c|c|c|}
\hline 73. & Chesterfield & Spireites, The & $\begin{array}{l}\text { Named for the famous crooked spire on } \\
\text { the local Church of St. Mary and All } \\
\text { Saints. }\end{array}$ \\
\hline 74. & Crewe Alexandra & $\begin{array}{l}\text { Railwaymen, } \\
\text { The }\end{array}$ & Crewe is a major railway junction. \\
\hline 75. & Fulham & Cottagers, The & $\begin{array}{l}\text { From the club's home ground, Craven } \\
\text { Cottage }\end{array}$ \\
\hline 76. & Reading & Royals, The & $\begin{array}{l}\text { The town is in the Royal County of } \\
\text { Berkshire. }\end{array}$ \\
\hline 77. & $\begin{array}{l}\text { Rotherham } \\
\text { United }\end{array}$ & Millers, The & After the club's home ground (Millmoor) \\
\hline 78. & $\begin{array}{l}\text { Sheffield } \\
\text { Wednesday }\end{array}$ & Owls, The & $\begin{array}{l}\text { Based on the name of the area in which } \\
\text { the club is based (Owlerton). After a } \\
\text { visitor to the city drew a cartoon } \\
\text { depicting Sheffield Wednesday as an owl }\end{array}$ \\
\hline 79. & Sunderland & $\begin{array}{l}\text { Black Cats, } \\
\text { The }\end{array}$ & $\begin{array}{l}\text { Named after the Black Cat Battery gun } \\
\text { based on the River Wear. }\end{array}$ \\
\hline 80. & Torquay United & Gulls, The & Seaside link? \\
\hline 81. & Brentford & Bees, The & $\begin{array}{l}\text { A misinterpretation of an early terrace } \\
\text { chant "Buck up Bs" }\end{array}$ \\
\hline 82. & $\begin{array}{l}\text { Accrington } \\
\text { Stanley }\end{array}$ & Stanley & Shortened name \\
\hline 83. & Aldershot Town & Shots, The & Short version of name \\
\hline 84. & $\begin{array}{l}\text { Blackburn } \\
\text { Rovers }\end{array}$ & Rovers, The & Shortened name \\
\hline 85. & Doncaster Rovers & Rovers, The & Shortened name \\
\hline 86. & Gillingham & Gills, The & Shortened version of the name \\
\hline 87. & $\begin{array}{l}\text { Hartlepool } \\
\text { United }\end{array}$ & Pools & $\begin{array}{l}\text { Refers to the twin towns of Hartlepool } \\
\text { and West Hartlepool prior to the } \\
\text { integration of the two in } 1967 .\end{array}$ \\
\hline 88. & Leeds United & United & \\
\hline 89. & Middlesbrough & Boro, & Shortened version of name \\
\hline 90. & Oldham Athletic & Latics, The & Short version of name (Athletic) \\
\hline 91. & Rochdale & Dale, The & Short version of name \\
\hline 92. & $\begin{array}{l}\text { Shrewsbury } \\
\text { Town }\end{array}$ & Shrews, The & Shortened version of Shrewsbury \\
\hline 93. & Swansea City & Swans, The & Shortened version of name? \\
\hline 94. & \begin{tabular}{|l|} 
Tottenham \\
Hotspur
\end{tabular} & Spurs, & Short version of Hotspur. \\
\hline 95. & Tranmere Rovers & Rovers, The & Shortened name \\
\hline 96. & Wigan Athletic & Latics, The & Shortening of Athletic \\
\hline 97. & $\begin{array}{l}\text { Wolverhampton } \\
\text { Wanderers }\end{array}$ & Wolves & shortening of the name \\
\hline
\end{tabular}




\section{References}

Aguiar, A., \& Baillargeon, R. (1999). 2.5-Month-Old Infants' Reasoning about When Objects Should and Should Not Be Occluded. Cognitive Psychology, 39, 116-157.

Aguiar, A., \& Baillargeon, R. (2002). Developments in Young Infants' Reasoning about Occluded Objects. Cognitive Psychology, 45, 267-336.

Ahrens, K. (2002). When Love is not Digested: Underlying Reasons for Source to Target Domain Pairings in the Contemporary Theory of Metaphor. In Y. E. Hsiao (Ed.), Proceedings of the Cognitive Linguistics Conference (pp. 273302). Taipei: Cheng-Chi University.

Ahrens, K. (2010). Mapping Principles for Conceptual Metaphors. In C. Lynne, A. Deignan, G. Low \& Z. Todd (Eds.), Researching and Applying Metaphor in the Real World (pp. 185-207). Amsterdam: John Benjamins.

AI-Sharafi, A. G. M. (2004). Textual Metonymy: A Semiotic Approach. New York: Palgrave Macmillan.

Aitchison, J. (2003). Words in the Mind: An Introduction to the Mental Lexicon Oxford/Malden, MA: Blackwell Pub.

Alač, M., \& Coulson, S. (2004). The Man, the Key, or the Car: Who or What is Parked out back. Cognitive Science Online, 2, 21-34.

Allan, K. L. (2008). Metaphor and Metonymy: A Diachronic Approach. Chichester, West Sussex, United Kingdom/Malden, MA: Blackwell Pub.

Allwood, J. (1981). On the Distinctions between Semantics and Pragmatics. In W. Klein \& W. Levelt (Eds.), Crossing the Boundaries in Linguistics (pp. 177189). Dordrecht: Reidel.

Allwood, J. (2003). Meaning Potentials and Context: Some Consequencies for the Analysis of Variation in Meaning. In H. Cuyckens, R. Dirven \& J. R. Taylor (Eds.), Cognitive Approaches to Lexical Semantics (pp. 29-64). Berlin: Mouton de Gruyter.

Anderson, H. (1973). Abductive and Deductive Change. Languaqe, 49, 765-793.

Anderson, M. L. (2003). Embodied Cognition: A Field Guide. Artificial Intelligence, 149(1), 91-130.

Anderson, M. L. (2007). How to Study the Mind: An Introduction to Embodied Cognition. In F. Santoianni \& C. Sabatano (Eds.), Brain Development in Learning Environments: Embodied and Perceptual Advancements (pp. 6582). Cambridge: Cambridge Scholars Press.

Anderson, M. L. (2008). Evolution, Embodiment and the Nature of the Mind. In B. Hardy-Valle \& N. Payette (Eds.), Beyond the Brain: Embodied, Situated \& Distributed Cognition (pp. 15-28). Cambridge: Cambridge Scholar's Press.

Arata, L. (2005). The Definition of Metonymy in Ancient Greece. Style, 39(1), 55-71.

Arterberry, M. E., \& Bornstein, M. H. (2001). Three-Month-Old Infants' Categorization of Animals and Vehicles Based on Static and Dynamic Attributes. Journal of Experimental Child Psychology, 80, 333-346.

Bahrick, L. E., Gogate, L. J., \& Ruiz, I. (2002). Attention and Memory for Faces and Actions in Infancy: The Salience of Actions over Faces in Dynamic Events. Child Development, 73(6), 1629-1643.

Bakhtin, M. M. (1981). The Dialogic Imagination: Four Essays (Michael Holquist, Ed. and Caryl Emerson and Michael Holquist Trans.). Austin, Tex.: University of Texas Press. 
Barcelona, A. (2000a). Introduction: The Cognitive Theory of Metaphor and Metonymy. In A. Barcelona (Ed.), Metaphor and Metonymy at the Crossroads (pp. 1-28). Berlin/New York: Mouton de Gruyter.

Barcelona, A. (2000b). Metaphor and Metonymy at the Crossroads: A Cognitive Perspective. Berlin/New York: Mouton de Gruyter.

Barcelona, A. (2000c). On the Plausibility of Claiming a Metonymic Motivation for Conceptual Metaphor. In A. Barcelona (Ed.), Metaphor and Metonymy at the Crossroads : A Cognitive Perspective (pp. 31-58). Berlin/New York: Mouton de Gruyter.

Barcelona, A. (2002a). Clarifying and Applying the Notions of Metaphor and Metonymy within Cognitive Linguistics: An Update. In R. Dirven \& R. Pörings (Eds.), Metaphor and Metonymy in Comparison and Contrast (pp. 207-277). Berlin/New York: Mouton de Gruyter.

Barcelona, A. (2002b). On the Ubiquity and Multiple-level Operation of Metonymy. In B. Lewandowska-Tomaszczyk \& K. Turewicz (Eds.), Cognitive Linguistics Today (pp. 207-224). Frankfurt/Main: Peter Lang.

Barcelona, A. (2003a). The Case for a Metonymic Basis of Pragmatic Inferencing-Evidence from Jokes and Funny Anecdotes. In K.-U. Panther \& L. L. Thornburg (Eds.), Metonymy and Pragmatic Inferencing (pp. 61-102). Amsterdam/Philadelphia: John Benjamins.

Barcelona, A. (2003b). Metonymy in Cognitive Linguistics: An Analysis and a Few Modest Proposals. In H. Cuyckens, T. Berg, R. Dirven \& K.-U. Panther (Eds.), Motivation in Language --- Studies in Honor of Günter Radden (pp. 223-255). Amsterdam/Philadelphia: John Benjamins.

Barcelona, A. (2004). Metonymy behind Grammar: The Motivation of the Seemingly "Irregular" Grammatical Behavior of English Paragon Names. In G. Radden \& K.-U. Panther (Eds.), Studies in Linguistic Motivation (pp. 357374). Berlin/New York: Mouton de Gruyter.

Barcelona, A. (2005a). The Multilevel Operation of Metonymy in Grammar and Discourse --- with Particular Attention to Metonymic Chains. In F. J. Ruiz de Mendoza Ibáñez \& M. S. Péna Cervel (Eds.), Cognitive Linguistics: Internal Dynamics and Interdisciplinary Interaction (pp. 313-352). Berlin/New York: Mouton de Gruyter.

Barcelona, A. (2005b). The Role of Metonymy in Discourse-Pragmatic Inferencing.

In J. L. J. L. Otal, I. Navarro i Ferrando \& B. Bellés Fortuño (Eds.),

Cognitive and Discourse Approaches to Metaphor and Metonymy (pp. 29-44).

Castellón de la Plana: Universitat Jaume I.

Barcelona, A. (2007a). The Multi-Level Role of Metonymy in Grammar and

Discourse: A Case Study. In K. Kosecki (Ed.), Perspectives on Metonymy

(pp. 103-131). Berlin /New York /Oxford Peter Lang.

Barcelona, A. (2007b). The Role of Metonymy in Meaning Construction at

Discourse Level: A Case Study. In G. Radden, K.-M. Köpcke, T. Berg \& P.

Siemund (Eds.), Aspects of Meaning Construction (pp. 51-75).

Amsterdam/Philadelphia: John Benjamins.

Barcelona, A. (2009). Motivation of Construction Meaning and Form. In K.-U.

Panther, L. L. Thornburg \& A. Barcelona (Eds.), Metonymy and Metaphor in Grammar (pp. 363-401). Amsterdam: John Benjamins.

Barcelona, A. (2011). Reviewing the Properties and Prototype Structure of

Metonymy. In R. Benczes, A. Barcelona \& F. Ruiz de Mendoza (Eds.), 
Defining Metonymy in Cognitive Linguistics: Towards a Consensus View (pp. 7-57). Amsterdam: John Benjamins.

Barlow, M., \& Kemmer, S. (Eds.). (2000). Usage-based models of language.

Stanford: CSLI Publications.

Barnden, J. A. (2010). Metaphor and Metonymy: Making Their Connections More Slippery. Cognitive Linguistics, 21(1), 1-34.

Barsalou, L. W. (1999a). Perceptions of Perceptual Symbols. Behavioral and Brain Sciences, 22(4), 637-661.

Barsalou, L. W. (1999b). Perceptual Symbol Systems. Behavioral and Brain Sciences, 22(04), 577-660. doi: doi:10.1017/S0140525X99002149

Barsalou, L. W. (2003). Abstraction in Perceptual Symbol Systmes. Philosophical Transactions of the Royal Society B, 358, 1177-1187.

Barsalou, L. W. (2008a). Grounded Cognition. Annual Review of Psychology, 59, 617-645.

Barsalou, L. W. (2008b). Grounding Symbolic Operations in the Brain's Modal

Systems. In G. R. Semin \& E. R. Smith (Eds.), Embodied Grounding: Social, Cognitive, Affective, and Neuroscientific Approaches (pp. 9-42). New York:

Cambridge University Press.

Barsalou, L. W. (2008c). Situating Concepts. In P. Robbins \& M. Aydede (Eds.), Cambridge Handbook of Situated Cognition (pp. 236-263). New York:

Cambridge University Press.

Barsalou, L. W. (2010). Grounded Cognition: Past, Present, and Future. Topics in Cognitive Science, 2(4), 716-724.

Barsalou, L. W., \& Wiemer-Hastings, K. (2005). Situating Abstract Concepts. In D.

Pecher \& R. A. Zwaan (Eds.), Grounding Cognition--- The Role of

Perception and Action in Memory, Language, and Thinking (pp. 128-163).

Cambridge: Cambridge University Press.

Bauer, P. J., \& Mandler, J. M. (1989). One Thing Follows Another: Effects of

Temporal Structure on 1- to 2- Year-Olds' Recall of Events. Developmental Psychology, 25(2), 197-206.

Bauer, P. J., \& Mandler, J. M. (1992). Putting the Horse Before the Cart: The Use of Temporal Order in Recall of Events by One-Year-Old Children.

Developmental Psychology, 28(3), 441-452.

Behl-Chadha, G. (1996). Basic-level and Superordinate-like Categorical Representations in Early Infancy. Cognition, 60, 105-141.

Benczes, R. (2009). Meaning-making: The Bigger Picture -- An Interview with Zoltán Kövecses. Annual Review of Cognitive Linguistics, 7, 291-300.

Berlucchi, G., \& Aglioti, S. (1997). The Body in the Brain: Neural Bases of Corporeal Awareness. Trends in Neurosciences, 20(12), 560-564.

Blakemore, D. (1992). Understanding Utterances Oxford, UK/Cambridge, Mass., USA: Blackwell.

Blank, A. (1999). Co-presence and Succession: A Cogntive Typology of Metonymy. In K.-U. Panther \& G. Radden (Eds.), Metonymy in Language and Thought (pp. 169-191). Amsterdam/Philadelphia: J. Benjamins.

Blank, A., \& Koch, P. (Eds.). (1999). Historical Semantics and Cognition. Berlin: Mouton de Gruyter.

Boroditsky, L. (2000). Metaphoric Structuring: Understanding Time through Spatial Metaphors. Cognition, 75(1), 1-28.

Boroditsky, L. (2001). Does Language Shape Thought? Mandarin and English Speakers' Conceptions of Time. Cognitive Psychology, 43(1), 1-22. 
Boroditsky, L. (2002). Mental Representations of Abstract Domains. Dissertation Abstracts International: Section B: The Sciences and Engineering, 62(9-B), 4246.

Boroditsky, L. (2009). How does our Language Shape the Way We Think? In M. Brockman (Ed.), What's Next: Dispatches on the Future of Science (pp. 116129). New York: Vintage Books.

Boroditsky, L., \& Ramscar, M. (2002). The Roles of Body and Mind in Abstract Thought. Psychological Science, 13(2), 185-189.

Boroditsky, L., Scmidt, L. A., \& Phillips, W. (2003). Sex, Syntax and Semantics. In D. Gentner \& S. Goldin-Meadow (Eds.), Language in Mind: Advances in the Study of Language and Thought (pp. 61-78). Cambridge, MA: MIT Press.

Brdar-Szabó, R., \& Brdar, M. (2011). What do Metonymic Chains Reveal about the Nature of Metonymy? In R. Benczes, A. Barcelona \& F. Ruiz de Mendoza (Eds.), Defining Metonymy in Cognitive Linguistics: Towards a Consensus View (pp. 217-248). Amsterdam: John Benjamins.

Brdar, M. (2007). Metonymy in Grammar: Towards Motivating Extensions of Grammatical Categories and Constructions. Osijek: Faculty of Philosopny, Josip Juraj Strossmayer University.

Bredin, H. (1984). Metonymy. Poetics Today, 5(1), 45-58.

Brinton, L. J., \& Traugott, E. C. (2005). Lexicalization and Language Change. Cambridge: Cambridge University Press.

Brown, P., \& Levinson, S. C. (1987). Politeness: Some Universals in Language Usage. Cambridge: Cambridge University Press.

Burling, R. (1999). The Cognitive Prerequisites for Language. Psycholoquy (electronic journal), 10(032).

Campo, J. L. O. (2005). Cognitive and Discourse Approaches to Metaphor and Metonymy Castelló: Universidad Jaume I,.

Carroll, J. B. (1964). Language and Thought. London: PRENTICE-HALL.

Casasanto, D. (2009). Embodiment of Abstract Concepts: Good and Bad in Rightand Left-handers. Journal of Experimental Psychology: General, 138(3), 351-367.

Casasanto, D., \& Boroditsky, L. (2008). Time in the Mind: Using Space to Think about Time. Cognition, 106(2), 579-593.

Casasanto, D., Fotakopoulou, O., \& Boroditsky, L. (2010). Space and Time in the Child's Mind: Evidence for a Cross-dimensional Asymmetry. Cognitive Science, 34(3), 387-405.

Chen, G. (2000). The Grammaticalization of Concessive Markers in Early Modern English. In O. Fischer, A. Rosenbach \& D. Stein (Eds.), Pathways of Change: Grammaticalization in English (pp. 85-110). Amsterdam/Philadelphia: John Benjamins.

Chiang, W.-C., \& Wynn, K. (2000). Infants' Tracking of Objects and Collections. Cognition, 77, 169-195.

Chiel, H. J., \& Beer, R. D. (1997). The Brain Has a Body: Adaptive Behavior Emerges from Interactions of Nervous System, Body and Environment. Trends in Neurosciences, 20(12), 553-557.

Ciepiela, K. (2007). Metonymy in Aphasia. In K. Kosecki (Ed.), Perspective on Metonymy (pp. 199-208). Berlin/New York/Oxford: Peter Lang.

Clark, E. V., \& Clark, H. H. (1979). When Nouns Surface as Verbs. Language, 55(4), 767-811. 
Clark, H. H. (1992). Arenas of Language Use. Chicago: The University of Chicago Press.

Clark, H. H. (1996). Using Language. Cambridge: Cambridge University Press.

Clark, H. H., \& Gerrig, R. J. (1983). Understanding Old Words with New Meanings. Journal of Verbal Learning and Verbal Behavior, 22, 591-608.

Clausner, T. C. (2005). Image Schema Paradoxes: Implications for Cognitive Semantics. In B. Hampe (Ed.), From Perception to Meaning: Image Schemas in Cognitive Linguistics (pp. 93-110). Berlin: Walter de Gruyter.

Corina, D., Kritchevsky, M., \& Bellugi, U. (1996). Visual Language Processing and Unilateral Neglect: Evidence from American Sign Language. Cognitive Neuropsychology, 13(3), 321-356.

Cornelissen, J. P. (2008). Metonymy in Language about Organizations: A CorpusBased Study of Company Names. Journal of Management Studies, 45(1), 7999. doi: 10.1111/j.1467-6486.2007.00737.x

Coulson, S., \& Oakley, T. (2000). Blending Basics. Cognitive Linguistics, 11(3-4), 175-196.

Coulson, S., \& Oakley, T. (2003). Metonymy and Conceputal Blending. In K.-U. Panther \& L. L. Thornberg (Eds.), Metonymy and Pragmatic Inferencing (pp. 59-88). Amsterdam: John Benjamins.

Cowart, M. (2009). Embodied Cognition. The Internet Encyclopedia of Philosophy Retrieved April 22, 2009, from http://www.iep.utm.edu/e/embodcog.htm

Croft, W. (2002). The Role of Domains in the Interpretation of Metaphors and Metonymies. In René Dirven \& R. Pörings (Eds.), Metaphor and Metonymy in Comparison and Contrast (pp. 161-206). Berlin/New York: Mouton de Gruyter.

Croft, W., \& Cruse, D. A. (2004). Cognitive Linguistics. Cambridge, UK/New York: Cambridge University Press.

Cruse, D. A. (1999). Meaning in Language: An Introduction to Semantics and Pragmatics. Oxford/New York: Oxford University Press.

Crystal, D. (1992). An Encyclopedic Dictionary of Language and Languages. Oxford: Blackwell.

Cuyckens, H., Berg, T., Dirven, R., \& Panther, K.-U. (Eds.). (2003). Motivation in Language --- Studies in Honor of Günter Radden. Amsterdam/Philadelphia: John Benjamins.

Cuyckens, H., Dirven, R., \& Taylor, J. R. (Eds.). (2003). Cognitive Approaches to Lexical Semantics. Berlin: Mouton de Gruyter.

Cuyckens, H., \& Zawada, B. (Eds.). (2001). Polysemy in Cognitive Linguistics. Amsterdam: John Benjamins.

Deignan, A. (2006). The Grammar of Linguistic Metaphors. In A. Stefanowitsch \& S. T. Gries (Eds.), Corpus-based Approaches to Metaphor and Metonymy (pp. 106-122). Berlin: Mouton de Gruyter.

Delpit, L. D. (1988). The Silenced Dialogue: Power and Pedagogy in Educating Other People's Children. Harvard Educational Review, 58(3), 280-298.

Dirven, R. (1999). Conversion as a Conceptual Metonymy of Basic Event Schemata. In K.-U. Panther \& G. Radden (Eds.), Metonymy in Language and Thought (pp. 275-287). Amsterdam/Philadelphia: John Benjamins.

Dirven, R. (2002). Metonymy and Metaphor: Different Mental Strategies of Conceptualization. In R. Dirven \& R. Pörings (Eds.), Metaphor and Metonymy in Comparison and Contrast (pp. 75-111). Berlin/New York: Mouton de Gruyter. 
Dirven, R., \& Pörings, R. (Eds.). (2002). Metaphor and Metonymy in Comparison and Contrast. Berlin/New York: Mouton de Gruyter.

Dirven, R., \& Verspoor, M. (Eds.). (1998). Cognitive Exploration of Language and Linguistics Amsterdam/Philadelphia: John Benjamins.

Dodge, E., \& Lakoff, G. (2005). Image Schemas: From Linguistic Analysis to Neural Grounding. In B. Hampe (Ed.), From Perception to Meaning: Image Schemas in Cognitive Linguistics (pp. 57-92). Berlin: Walter de Gruyter.

Eco, U. (1979). The Role of the Reader: Explorations in the Semiotics of Texts. Bloomington: Indiana University Press.

Engel, S. (1999). Context is Everything: The Nature of Memory. New York: W. H. Freeman and Company.

Evans, V. (2006). Lexical Concepts, Cognitive Models and Meaning-construction. Cognitive Linguistics, 17(4), 491-534.

Evans, V. (2007). Towards a Cognitive Compositional Semantics: An Overview of LCCM theory. In U. Magnusson, H. Kardela \& A. Glaz (Eds.), Further Insights into Semantics and Lexicography (pp. 11-42). Lublin: Wydawnictwo UMCS.

Evans, V. (2009). How Words Mean. London: Oxford University Press.

Evans, V. (2010a). Cognitive Linguisitcs. In L. Cummings (Ed.), The Pragmatics Encyclopedia (pp. 46-49). London \& New York: Routledge.

Evans, V. (2010b). Figurative Language Understanding in LCCM Theory. Cognitive Linguistics, 21(4), 601-662.

Evans, V., Bergen, B. K., \& Zinken, J. (2007a). The Cognitive Linguistics Enterprise: An Overview. In V. Evans, B. K. Bergen \& J. Zinken (Eds.), The Cognitive Linguistics Reader (pp. 2-36). London/Oakville: Equinox.

Evans, V., Bergen, B. K., \& Zinken, J. (2007b). The Cognitive Linguistics Reader. London/Oakville: Equinox.

Evans, V., \& Green, M. (2006). Cognitive Linguistics: An Introduction. Edinburgh: Edinburgh University Press Ltd.

Evans, V., \& Zinken, J. (to appear). Figurative Language in a Modern Theory of Meaning Construction: A Lexical Concepts and Cognitive Models Approach. In C. M. a. R. Chrisley (Ed.), Art, Body and Embodiment. Cambridge: Cambridge Scholars Press.

Fagan Iii, J. F., \& Twarog Singer, L. (1979). The Role of Simple Feature Differences in Infants' Recognition of Faces. [doi: DOI: 10.1016/S0163-6383(79)800065]. Infant Behavior and Development, 2, 39-45.

Fang, Y., \& Zeng, D. (2007a). A Cognitive Approach to the Grammaticalization of Duoshao. Studies in Language and Linguistics, 27(3), 76-81.

Fang, Y., \& Zeng, D. (2007b). The Grammaticalization and Subjectivization of the Antonymous Compound Haodai. Journal of Zhejiang University (Humanities and Social Sciences), 37(1), 64-71.

Fauconnier, G. (1997). Mappings in Thought and Language. Cambridge: Cambridge University Press.

Fauconnier, G. (2002). Cognitive Linguistics. In L. Nadel (Ed.), Encyclopedia of Cognitive Science (pp. 539-543). Basingstoke: Palgrave Macmillan.

Fauconnier, G., \& Turner, M. (1999). Metonymy and Conceptual Integration. In K.U. Panther \& G. Radden (Eds.), Metonymy in Language and Thought (pp. 77-90). Amsterdam/Philadelphia: John Benjamins.

Feldman, J., \& Narayanan, S. (2004). Embodied Meaning in a Neural Theory of Language. Brain and Language, 89, 385-392. 
Feyaerts, K. (1999). Metonymic Hierarchies: The Conceptualization of Stupidity in German Idiomatic Expressions. In K.-U. Panther \& G. Radden (Eds.), Metonymy in Language and Thought (pp. 309-332). Amsterdam/Philadelphia:

J. Benjamins.

Feyaerts, K. (2000). Refining the Inheritance Hypothesis: Interaction between Metaphoric and Metonymic Hierarchies. In A. Barcelona (Ed.), Metaphor and Metonymy at the Crossroads : A Cognitive Perspective (pp. 59-78). Berlin/New York: Mouton de Gruyter.

Fillmore, C. J. (2006(1972)). Frame Semantics. In D. Geeraerts (Ed.), Cognitive Linguistics: Basic Readings (pp. 373-400). Amsterdam: Mouton de Gruyter.

Fischer, O. (2000). Grammaticalization: Unidirectional, Non-reversable? --- The Case of to Before the Infinitive in English. In O. Fischer, A. Rosenbach \& D. Stein (Eds.), Pathways of Change: Grammaticalization in English (pp. 149169). Amsterdam: John Benjamins.

Fischer, O., Norde, M., \& Perridon, H. (Eds.). (2004). Up and Down the Cline-- The Nature of Grammaticalization. Amsterdam/Philadelphia: John Benjamins.

Fischer, O., Rosenbach, A., \& Stein, D. (Eds.). (2000). Pathways of Change: Grammaticalization in English. Amsterdam/Philadelphia: John Benjamins.

Fisiak, J. (Ed.). (1985). Historical Semantics - Historical Word-formation. Berlin: Mouton de Gruyter.

Frank, R. M., Dirven, R., \& Ziemke, T. (Eds.). (2008). Body, Lanugage and Mind Volume 2: Sociocultural Situatedness. Berlin: Mouton de Gruyter.

Frye, D., Rawling, P., Moore, C., \& Myers, I. (1983). Object-person Discrimination and Communication at 3 and 10 Months. [doi: DOI: 10.1037/00121649.19.3.303]. Developmental Psychology, 19(3), 303-309.

Fu, X. (2005). On Properties of Speech Act. Social Sciences in Nanjing, 206(4), 8186.

Fu, X. (2006). Guan Yu Gou Shi You + VP (On the Construction of You + VP). Journal of China University of Geosciences (Social Sciences Edition), 6(5), 97-103, 108.

Fu, X. (2007). "You + VP" Yan Jiu Zong Shu (A Review of the Study on "You + $\left.V P^{\prime \prime}\right)$. Newsletter for Research in Chinese Studies, 26(3), 1-9.

$\mathrm{Fu}, \mathrm{X}$. (2010). Review on Metaphor, Metonymy, and Experientialist Philosophy Challenging Cognitive Semantics by Verena Haser, Mouton de Gruyter, Berlin/New York, 2005. International journal of Mind, Brain \& Cognition, 1(1), 115-118.

Gallagher, S. (2005). How the Body Shapes the Mind. Oxford: Oxford University Press.

Gallese, V., \& Lakoff, G. (2005). The Brain's Concepts: The Role of the Sensorymotor System in Conceptual Knowledge. Cognitive Neuropsychology, 22(3/4), 455-479.

Gardner, H. (1985). The Mind's New Science --- A History of the Cognitive Revolution. New York: Basic Books.

Geeraerts, D. (1988). Where does Prototypicality Come from? In B. Rudzka-ostyn (Ed.), Topics in Cognitive Linguistics (pp. 207-229).

Amsterdam/Philadelphia: John Benjamins.

Geeraerts, D. (1989). Prospects and Problems of Prototype Theory. Linguistics, 27, 587-612. 
Geeraerts, D. (1990). The Lexicographical Treatment of Prototypical Polysemy. In S. L. Tsohatzidis (Ed.), Meanings and Prototypes-- Studies in Lingusitic Categorization (pp. 195-210). London/New York: Routledge.

Geeraerts, D. (1997). Diachronic Prototype Semantics. Oxford: Clarendon Press.

Geeraerts, D. (2006a). Introduction: A Rough Guide to Cognitive Linguistics. In D. Geeraerts (Ed.), Cognitive Linguistics: Basic Readings (pp. 1-28). Berlin: Mouton de Gruyter.

Geeraerts, D. (Ed.). (2006b). Cognitive Linguistics - Basic Readings. Berlin: Walter de Gruyter.

Geeraerts, D., \& Cuyckens, H. (2007a). Introducing Cognitive Linguistics. In D. Geeraerts \& H. Cuyckens (Eds.), The Oxford Handbook of Cognitive Linguistics (pp. 3-21). Oxford: The Oxford University Press.

Geeraerts, D., \& Cuyckens, H. (2007b). The Oxford Handbook of Cognitive Linguistics. Oxford/New York: Oxford University Press.

Geeraerts, D., \& Peirsman, Y. (2011). Zones, Facets and Prototype-based Metonymy. In R. Benczes, A. Barcelona \& F. Ruiz de Mendoza (Eds.), Defining Metonymy in Cognitive Linguistics: Towards a Consensus View. Amsterdam: John Benjamins.

Gelb, M. J. (1998). How toThink like Leonardo da Vinci: Seven Steps to Genius Every Day. New York: Delacorte Press.

Gentner, D., \& Goldin-Meadow, S. (Eds.). (2003). Language in Mind: Advances in the Study of Language and Thought. Cambridge, Massachusetts: The MIT Press.

Gentner, D., Imai, M., \& Boroditsky, L. (2002). As Time Goes by: Evidence for Two Systems in Processing Space Time Metaphors. Language and Cognitive Processes, 17(5), 537-565.

Gibbs, R. W. (1994). The Poetics of Mind: Figurative Thought, Language, and Understanding. Cambridge [England]/New York, USA: Cambridge University Press.

Gibbs, R. W. (2005). The Psychological Status of Image Schemas. In B. Hampe (Ed.), From Perception to Meaning: Image Schemas in Cognitive Linguistics (pp. 113-136). Berlin: Walter de Gruyter.

Gibbs, R. W. (2006). Embodiment and Cognitive Science. Cambridge: Cambridge University Press.

Gibbs, R. W. (2006). Introspection and Cognitive Linguistics. Annual Review of Cognitive Linguistics, 4, 135-151.

Gibbs, R. W., \& Colston, H. L. (1995). The Cognitive Psychological Reality of Image Schemas and Their Transformations. Cognitive Linguistics, 6(4), 347378.

Gibbs, R. W., \& Matlock, T. (2001). Psycholinguistic Perspectives on Polysemy. In H. Cuyckens \& B. Zawada (Eds.), Polysemy in Cognitive Linguistics (pp. 213-239). Amsterdam/Philadelphia: John Benjamins.

Givón, T. (1971). Historical Syntax and Synchronic Morphology: An Archaeologist's Field Trip. Chicago Linguistic Society, 7, 394-415.

Glenberg, A. M., Havas, D., Becker, R., \& Rinck, M. (2005). Grounding Language in Bodily States. In D. Pecher \& R. A. Zwaan (Eds.), Grounding Cognition --The Role of Perception and Action in Memory, Language, and Thinking (pp. 115-128). Cambridge: Cambridge University Press.

Glucksberg, S. (2001). Understanding Figurative Language: From Metaphors to Idioms. Oxford: Oxford University Press. 
Glucksberg, S., \& Keysar, B. (1993). How Metaphors Work. In A. Ortony (Ed.), Metaphor and Thought (2 ed., pp. 401-424). Cambridge: Cambridge University Press.

Goldstone, R. L., \& Barsalou, L. W. (1998). Reuniting Perception and Conception. Cognition, 65(2-3), 231-262.

Gong, S.-P., Ahrens, K., \& Huang, C.-R. (2008). Chinese Word Sketch and Mapping Principles: A Corpus-Based Study of Conceptual Metaphors Using the BUILDING Source Domain. International Journal of Computer Processing of Oriental Languages, 21(2), 3-17.

Goossens, L. (1989). Metonymy in Metaphorization: From Body Parts (and Other Donor Domains) to Linguistic Action Duisburg: L.A.U.D.,.

Goossens, L. (1995). By Word of Mouth: Metaphor, Metonymy, and Linguistic Action in a Cognitive Perspective. Amsterdam/Philadelphia: J. Benjamins Pub. Co.

Goossens, L. (2000). Patterns of Meaning Extension, "Parallel Chaining", Subjectification, and Modal Shifts. In A. Barcelona (Ed.), Metaphor and Metonymy at the Crossroads: A Cognitive Perspective (pp. 149-170). Berlin/New York: Mouton de Gruyter.

Goossens, L. (2002). Metaphtonymy: The Interaction of Metaphor and Metonymy in Expressions for Linguistic Action. In R. Dirven \& R. Pörings (Eds.), Metaphor and Metonymy in Comparison and Contrast (pp. 349-378). Berlin/New York: Mouton de Gruyter.

Gorfein, D. S. (Ed.). (2001). On the Consequences of Meaning Selection:

Perspectives on Resolving Lexical Ambiguity. Washington, D.C.: American Psychological Association.

Grady, J. E. (1997). THEORIES ARE BUILDINGS Revisited. Cognitive Linguistics, 8(4), 267-290.

Grice, P. (1991). Studies in the Way of Words. London: Harvard University Press. Group $\mu$. (1970). Rhtoric Généale. Paris: Larousse.

Gumperz, J. J., \& Levinson, S. C. (Eds.). (1996). Rethinking Linguistic Relativity. Cambridge: Cambridge University Press.

Hampe, B. (2005a). Image Schemas in Cognitive Linguistics: Introduction. In B. Hampe (Ed.), From Perception to Meaning: Image Schemas in Cognitive Linguistics (pp. 1-12). Berlin: Walter de Gruyter.

Hampe, B. (Ed.). (2005b). From Perception to Meaning: Image Schemas in Cognitive Linguistics. Berlin: Walter de Gruyter.

Hanks, P. (2006). Nicknames. In K. Brown \& e. al (Eds.), Encyclopedia of Languages and Lingusitics (2 ed., Vol. 8, pp. 624-626). Oxford: Elsevier.

Haser, V. (2005). Metaphor, Metonymy, and Experientialist Philosophy: Challenging Cognitive Semantics. Berlin/New York: Mouton de Gruyter.

Haspelmath, M. (1989). From Purposive to Infinitive--- A Universal Path of Grammaticalization. Folia Linguistica Historica, 10, 287-310.

Haspelmath, M. (2004). On Directionality in Language Change with Particular Reference to Grammaticalization. In O. Fischer, M. Norde \& H. Perridon (Eds.), Up and Down the Cline - The Nature of Grammaticalization (pp. 1744). Amsterdam: John Benjamins.

Hauk, O., Johnsrude, I., \& Pulvermüler, F. (2004). Somatotopic Representation of Action Words in Human Motor and Premotor Cortex. Neuron, 41, 301-307.

Hein, G. E. (1999). Is Meaning Making Constructivism? Is Constructivism Meaning Making? The Exhibitionist, 18(2), 15-18. 
Heine, B. (1993). Auxiliaries: Cognitive Forces and Grammaticalization. New York: Oxford University Press.

Heine, B. (1997a). Possession: Cognitive Sources, Forces, and Grammaticalization. Cambridge: Cambridge University Press.

Heine, B. (1997b). Possession: Cognitive Sources, Forces, and Grammaticalization Cambridge/New York: Cambridge University Press.

Heine, B., Claudi, U., \& Hunnemeyer, F. (1991). Grammaticalization: A Conceptual Framework Chicago: University of Chicago Press.

Heine, B., Claudi, U., \& Hünnemeyer, F. (1991). From Cognition to Gramamr: Evidence from African Languages. In E. C. Traugott \& B. Heine (Eds.), Approaches to Grammaticalization: Volume I. Theoretical and Methodological Issues (pp. 149-188). Amsterdam: John Benjamins.

Hespos, S. J., \& Baillargeon, R. (2001). Reasoning about Containment Events in Very Young Infants. Cognition, 78, 207-245.

Holmes, J., Vine, B., \& Johnson, G. (1998). Guide to The Wellington Corpus of Spoken New Zealand English Wellington: School of Linguistics and Applied Language Studies, Victoria University of Wellington.

Hopper, P. J., \& Traugott, E. C. (1993). Grammaticalization. Cambridge [England]/New York, USA: Cambridge University Press.

Hopper, P. J., \& Traugott, E. C. (2003). Grammaticalization (2 ed.). Cambridge: Cambridge University Press.

Hubbard, E., Piazza, M., Pinel, P., \& Dehaene, S. (2005). Interactions between Numbers and Space in Parietal Cortex. Nature Reviews: Neuroscience, 6(6), 435-448.

Hubbard, E., \& Teuscher, U. (2010). Neural Constraints on Temporal-Spatial Metaphors. SSRN eLibrary.

Iljic, R. (2009). Grammaticalization of the Notion of "Passing" in Chinese (Aspectual Values). Bulletin of the School of Oriental and African Studies, 72(03), 513-524. doi: doi:10.1017/S0041977X0999005X

Jakobson, R. (1971a). Aphasia as a Linguistic Topic Selected Writings (pp. 229-238). The Hague/Paris: Mouton.

Jakobson, R. (1971b). Two Aspects of Language and Two Types of Aphasic Disturbances Selected Writings (pp. 239-259). The Hague/Paris: Mouton.

Jakobson, R. (1971/2002). The Metaphoric and Metonymic Poles. In R. Dirven \& R. Pörings (Eds.), Metaphor and Metonymy in Comparison and Contrast. Berlin/New York: Mouton de Gruyter.

Janda, L. A. (2006). Cognitive Linguistics. Glossos, 8. Retrieved from

Janssen, T. A. J. M. (2003). Monosemy versus Polysemy. In H. Cuyckens, R. Dirven \& J. R. Taylor (Eds.), Cognitive Approaches to Lexical Semantics (pp. 93122). Berlin/New York: Mouton de Gruyter.

Johnson, M. (1987). The Body in the Mind: The Bodily Basis of Meaning, Imagination, and Reasoning. Chicago: University of Chicago Press.

Johnson, M. (2005). The Philosophical Significance of Image Schemas. In B. Hampe (Ed.), From Perception to Meaning: Image Schemas in Cognitive Linguistics (pp. 15-34). Berlin: Walter de Gruyter.

Johnson, M. (2007). The Meaning of the Body: Aesthetics of Human Understanding. Chicago: University of Chicago Press.

Johnson, M., \& Lakoff, G. (2002). Why Cognitive Linguistics Requires Embodied Realism. Cognitive Linguistics, 13(3), 245-263. 
Kalisz, R. (2007). Metonymy and Semantic Representations. In K. Kosecki (Ed.), Perspectives on Metonymy (pp. 31-41). Berlin/New York/Oxford: Peter Lang.

Katz, A. N., Cacciari, C., Gibbs, R. W. J., \& Turner, M. (Eds.). (1998). Figurative Language and Thought. Oxford: Oxford University Press.

Kemmer, S., \& Barlow, M. (2000a). Introduction: A Usage-based Conception of Language. In S. Kemmer \& M. Barlow (Eds.), Usage-based Models of Language. Stanford: CSLI Publications.

Kemmer, S., \& Barlow, M. (Eds.). (2000b). Usage-based Models of Language. Stanford: CSLI Publications.

Knapp, H. P., \& Corina, D. P. (2010). A Human Mirror Neuron System for Language: Perspectives from Signed Languages of the Deaf. [doi: DOI: 10.1016/j.bandl.2009.04.002]. Brain and Language, 112(1), 36-43.

Kobayashi, H. (1997). The Role of Actions in Making Inferences about the Shape and Material of Solid Objects among Japanese 2 Year-old Children. Cognition, 63, 251-269.

Koch, P. (1999). Frame and Contiguity: On the Cognitive Bases of Metonymy and Certain Types of Word Formation. In K.-U. Panther \& G. Radden (Eds.), Metonymy in Language and Thought (pp. 139-167). Amsterdam/Philadelphia: J. Benjamins.

Koch, P. (2001). Metonymy: Unity in Diversity. Journal of Historical Pragmatics, 2(2), 201-244.

Koch, P. (2004). Metonymy between Pragmatics, Reference, and Diachrony. Metaphorik.de(07), 6-54.

Kosslyn, S. M., Ganis, G., \& Thompson, W. L. (2001). Neural Foundations of Imagery. Nature Reviews: Neuroscience, 2, 635-642.

Kotovsky, L., \& Baillargeon, R. (1994). Calibration-Based Reasoning about Collision Events in 11-Month-Old Infants. Cognition, 51, 107-129.

Kotovsky, L., \& Baillargeon, R. (1998). The Development of Calibration-based Reasoning about Collision Events in Young Infants. Cognition, 67, 311-351.

Kövecses, Z. (2000). The Scope of Metaphor. In A. Barcelona (Ed.), Metaphor and Metonymy at the Crossroads: A Cognitive Perspective (pp. 79-92). Berlin/New York: Mouton de Gruyter.

Kövecses, Z. (2005). A Broad View of Cognitive Linguisitcs. Acta Lnguistica Hungarica, 52(2-3), 135-172.

Kövecses, Z. (Ed.). (2008). The Conceptual Structure of Happiness. Helsinki: Helsinki Collegium for Advanced Studies. .

Kövecses, Z., \& Radden, G. (1998). Metonymy: Developing a Cognitive Linguistic View. Cognitive Linguistics, 9(1), 37-77.

Krauss, S. E. (2005). Research Paradigms and Meaning Making: A Primer. The Qualitative Report, 10(4), 758-770.

Kristiansen, G., Achard, M., \& Dirven, R. (Eds.). (2006). Cognitive Linguistics: Current Applications and Future Perspectives. Berlin: Walter de Gruyter.

Kurzman, C. (2008). Meaning-making in Social Movements: Introduction. Anthropological Quarterly, 81(1), 5-15.

Kuteva, T. (1991). The Auxiliarization Constraint and Reference. Unpublished Paper.

Kuteva, T. (2004). Auxiliation: An Enquiry into the Nature of Grammaticalization Oxford: Oxford University Press.

Kwiatkowska, A. (2007). Pre-linguisitc and Non-linguistic Metonymy. In K. Kosecki (Ed.), Perspectives on Metonymy (pp. 297-307). Berlin: Peter Lang. 
Lacey, S., Stilla, R., \& Sathian, K. (in press). Metaphorically Feeling:

Comprehending Textural Metaphors Activates Somatosensory Cortex. Brain and Language.

Lakoff, G. (1987a). Cognitive Models and Prototype Theory. In U. Neisser (Ed.), Concepts and Conceptual Development: Ecological and Intellectual Factors in Categorization (pp. 63-100). Cambridge: Cambridge University Press.

Lakoff, G. (1987b). Women, Fire, and Dangerous Things: What Categories Reveal about the Mind. Chicago: The University of Chicago Press.

Lakoff, G. (1990). The Invariance Hypothesis: Is Abstract Reason Based on Imageschemas? Cognitive Linguistics, 1(1), 39-74.

Lakoff, G. (2009). The Neural Theory of Metaphor. SSRN eLibrary.

Lakoff, G., \& Johnson, M. (1980). Metaphors We Live by. Chicago: Chicago University Press.

Lakoff, G., \& Johnson, M. (1999). Philosophy in the Fesh: The Embodied Mind and Its Challenge to Western Thought New York: Basic Books.

Lakoff, G., \& Turner, M. (1989). More than Cool Reason: A Field Guide to Poetic Metaphor. Chicago: The University of Chicago Press.

Landau, B. (2000). Language and Space. In B. Landau, J. Sabini, J. Jonides \& E. L. Newport (Eds.), Perception, Cognition, and Language (pp. 209-230). Cambridge: MIT.

Langacker, R. W. (1987). Foundations of Cognitive Grammar Vol. I: Theorectical Prerequisites. Stanford: Stanford University Press.

Langacker, R. W. (1991). Foundations of Cognitive Grammar, vol. 2, Descriptive Application. Stanford: Stanford: University Press.

Langacker, R. W. (1993). Reference-point Constructions. Cognitive Linguistics, 4(1), $1-38$.

Langacker, R. W. (1999). Grammar and Conceptualization. Berlin/New York: Mouton de Gruyter.

Langacker, R. W. (2004). Metonymy in Grammar. Journal of Foreign Languages, 6, 2-24.

Langacker, R. W. (2009). Metonymic Grammar. In K.-U. Panther \& L. L. Thornburg (Eds.), Metonymy and Metaphor in Grammar (pp. 45-71). Berlin: Mouton de Gruyter.

LaVelle, A. (2007). Metonymy: A Peircean Semiotic Categorization and Typologization in Relation to Other Tropes and Sign Types. $\mathrm{PhD} \mathrm{PhD}$, University of New Mexico.

Lee, D. (2001). Cognitive Linguistics: An Introduction. Victoria, Austria: Oxford University Press.

Lehmann, C. (2002). Thoughts on Grammaticalization Vol. 2. Erfurt: Arbeitspapiere des Seminars für Sprachwissenschaft der Universität Erfurt.

Leslie, A. M. (1988). The Necessity of Illusion: Perception and Thought in Infancy. In L. Weiskrantz (Ed.), Thought Without Language (pp. 185-210). Oxford: Clarendon Press.

López-Couso, M. a. J., \& Seoane, E. (Eds.). (2008). Rethinking Grammaticalization: New Perspectives. Amsterdam/Philadelphia: John Benjamins.

Mandler, J. M. (1983). Representation. In J. H. Flavell \& E. M. Markman (Eds.), Handbook of Child Psychology: Cognitive Development (4 ed., Vol. 3). New York: Wiley.

Mandler, J. M. (1988). How to Build a Baby: On the Development of an Accessible Representational System. Cognitive Development, 3, 113-136. 
Mandler, J. M. (1989). Categorization in Infancy and Early Childhood. In M. A. Luszcz \& T. Nettelbeck (Eds.), Psychological Development: Perspectives across the Life-span (pp. 127-139). Amsterdam: Elsevier.

Mandler, J. M. (1992). How to Build a Baby II: Conceptual Primitives. Psychological Review, 99, 587-604.

Mandler, J. M. (1993). On Concepts. Cognitive Development, 8(2), 141-148.

Mandler, J. M. (1994). From Perception to Conception. In P. V. Geert, L. P. Moss \& W. J. Baker (Eds.), Annals of Theoretical Psychology (Vol. 10, pp. 43-57). New York: Plenum Press.

Mandler, J. M. (1996). Preverbal Representation and Language. In P. Bloom, M. A. Peterson, L. Nadel \& M. F. Garrett (Eds.), Language and Space (pp. 365384). Cambridge: The MIT Press.

Mandler, J. M. (1997). Development of Categorization: Perceptual and Conceptual Categories. In G. Bremner, A. Slater \& G. Butterworth (Eds.), Infant Development: Recent Advances (pp. 163-189). Hove, East Sussex, UK: Psychology Press.

Mandler, J. M. (1998a). Babies Think Before They Speak. Human Development(41), 116-126.

Mandler, J. M. (1998b). The Rise and Fall of Semantic Memory. In M. A. Conway, S. E. Gathercole \& C. Cornoldi (Eds.), Theories of Memory (Vol. II, pp. 147169). Sussex: Psychology Press.

Mandler, J. M. (1999). Seeing is not the Same as Thinking: Commentary on "Making Sense of Infant Categorization". Developmental Review, 19, $297-$ 306.

Mandler, J. M. (2000). Perceptual and Conceptual Processes in Infancy. Journal of Cognition and Development, 1, 3-36.

Mandler, J. M. (2003). Conceptual Categorization. In D. H. Rakison \& L. M. Oakes (Eds.), Early Category and Concept Development: Making Sense of the Blooming, Buzzing Confusion (pp. 103-131). Oxford: Oxford University Press.

Mandler, J. M. (2004a). The Foundations of Mind: Origins of Conceptual Thought. Oxford: Oxford University Press.

Mandler, J. M. (2004b). Thought before Language. Trends in Cognitive Sciences, $8(11), 508-513$.

Mandler, J. M. (2005). How to Build a Baby III: Image Schemas and the Transition to Verbal Thought. In B. Hampe (Ed.), From Perception to Meaning: Image Schemas in Cognitive Linguistics (pp. 137-164). Berlin: Walter de Gruyter.

Mandler, J. M. (2006). Actions Organize the Infant's World. In K. HIrsh-Pasek \& R. M. Golinkoff (Eds.), Action Meets Word --- How Children Learn Verbs (pp. 111-133). Oxford: Oxford University Press.

Mandler, J. M. (2007a). The Conceptual Foundations of Animals and Artifacts. In E. Margolis \& S. Laurence (Eds.), Creations of the Mind: Theories of Artifacts and Their Representation (pp. 191-211). Oxford: Oxford University Press.

Mandler, J. M. (2007b). On the Origins of the Conceptual System. [doi: DOI: 10.1037/0003-066X.62.8.741]. American Psychologist, 62(8), 741-751.

Mandler, J. M. (2008). On the Birth and Growth of Concepts. Philosophical Psychology, 21(2), 207-230.

Mandler, J. M. (2010). The Spatial Foundations of the Conceptual System. Language and Cognition, 2(1), 21-44. 
Mandler, J. M., \& Bauer, P. J. (1988). The Cradle of Categorization: Is the Basic Level Basic? Cognitive Development, 3, 247-264.

Mandler, J. M., Bauer, P. J., \& McDonough, L. (1991). Separating the Sheep from the Goats: Differentiating Global Categories. Cognitive Psychology, 23, 263298.

Mandler, J. M., \& McDonough, L. (1993). Concept Formation in Infancy. Cognitive Development, 8, 291-318.

Mandler, J. M., \& McDonough, L. (1996). Drinking and Driving Don't Mix: Inductive Generalization in Infancy. Cognition, 59, 307-335.

Mandler, J. M., \& McDonough, L. (1998). Studies in Inductive Inference in Infancy. Cognitive Psychology, 37, 60-96.

Mandler, J. M., \& McDonough, L. (2000). Advancing downward to the Basic Level. Journal of Cognition and Development, 1(4), 379-403.

Markert, K., \& Hahn, U. (1997). On the Interaction of Metonymies and Anaphora. Paper presented at the Proceedings of the Fifteenth International Joint Conference on Artifical Intelligence - Volume 2, Nagoya, Japan.

Marsen, S. (2009). Is Meaning Information? Some Thoughts on Linguistic Ambiguity, Embodied Emotion and the Making of Meaning. Semiotica, 1741(4), 203-225.

Mashal, N., Faust, M., Hendler, T., \& Jung-Beeman, M. (2007). An fMRI Investigation of the Neural Correlates Underlying the Processing of Novel Metaphoric Expressions. Brain and Language, 100, 115-126.

Matlock, T., Ramscar, M., \& Boroditsky, L. (2005). On the Experiential Link Between Spatial and Temporal Language. Cognitive Science: A Multidisciplinary Journal, 29(4), 655-664.

McDonough, L., \& Mandler, J. M. (1998). Inductive Generalization in 9- and 11Month-Olds. Developmental Science, 1(2), 227-232.

Mittelberg, I., \& Waugh, L. (2008). Metonymy First, Metaphor Second: A Two-Step Model for the Interpretation of Metaphoric Gestures. SSRN eLibrary.

Mittelberg, I., \& Waugh, L. R. (2009). Metonymy First, Metaphor Second: A Cognitive-semiotic Approach to Multimodal Figures of Thought in Cospeech Gesture. In C. J. Forceville \& E. Urios-Aparisi (Eds.), Multimodal Metaphor (pp. 329-357). Berlin: Mouton de Gruyter.

Mkhatshwa, S. N. L. (1991). Metaphorical Extensions as a Basis for Grammaticalization: With Special Reference to Zulu Auxiliary Verbs. M.A., University of South Africa, Pretoria.

Morgan, J., O'Neil, C., \& Harré, R. (1979). Nicknames: Their Origins and Social Consequences. London: Routledge \& Kegan Paul.

Morrongiello, B. A., Fenwick, K. D., \& Chance, G. (1998). Crossmodal Learning in Newborn Infants: Inferences about Properties of Auditory-Visual Events. Infant Behavior and Development, 21(4), 543-554.

Mujic, B. K. (2009). Linguistic and Pictorial Metonymy in Advertising. In J. Valenzuela, A. Rojo \& C. Soriano (Eds.), Trends in Cognitive Linguistics: Theoretical and Applied Models. Frankfurt: Peter Lang.

Musson, G., \& Tietze, S. (2004). Places and Spaces: The Role of Metonymy in Organizational Talk. Journal of Management Studies, 41(8), 1301-1323. doi: 10.1111/j.1467-6486.2004.00476.x

Nelson, K. (2000). Global and Functional: Mandler's Perceptual and Conceptual Processes in Infancy. Journal of Cognition and Development, 1, 49-54. 
Nerlich, B. (2010). Synecdoche: A Trope, a Whole Trope, and Nothing but a Trope? In A. Burkhardt \& B. Nerlich (Eds.), Tropical Truth(s): The Epistemology of Metaphor and other Tropes (pp. 297-320). Berlin: De Gruyter.

Nerlich, B., \& Clarke, D. D. (2003). Polysemy and Flexibility: Introduction and Overview. In B. Nerlich, Z. Todd, V. Herman \& D. D. Clarke (Eds.), Polysemy: Flexible Patterns of Meaning in Mind and Language (pp. 3-30). Berlin: Mouton de Gruyter.

Nerlich, B., Todd, Z., Herman, V., \& Clarke, D. D. (Eds.). (2003). Polysemy: Flexible Patterns of Meaning in Mind and Language. Berlin: Mouton de Gruyter.

Nunberg, G. (1995). Transfers of Meaning. Journal of Semantics, 12, 109-132.

Oakley, T. (2007). Image Schemas. In D. Geeraerts \& H. Cuyckens (Eds.), The Oxford Handbook of Cognitive Linguistics (pp. 214-235). Oxford: Oxford University Press.

Ortony, A. (Ed.). (1993). Metaphor and Thought (2 ed.). Cambridge: Cambridge University Press.

Pankhurst, A. (1999). Recontextualization of Metonymy in Narrative and the Case of Morrison's Song of Solomon. In K.-U. Panther \& G. Radden (Eds.), Metonymy in Language and Thought (pp. 385-399). Amsterdam/Philadelphia: John Benjamins.

Panther, K.-U. (1994). Sentence Types, Speech Acts, and Metonymy. Hamburg: Seminar fur Englische Sprache and Kultur.

Panther, K.-U. (2003). Review of Metonymy, Grammar and Communication. Annual Review of Cognitive Linguistics, 1(1), 276-288.

Panther, K.-U. (2005). The Role of Conceptual Metonymy in Meaning Construction. In F. J. R. d. M. Ibáñez \& M. S. P. Cervel (Eds.), Cognitive Linguistics: Internal Dynamics and Interdisciplinary Interaction (pp. 353-386). Berlin: Mouton de Gruyter.

Panther, K.-U. (2006). Metonymy as a Usage Event. In G. Kristiansen, M. Achard, R. Dirven \& F. J. Ruiz de Mendoza Ibáñez (Eds.), Cognitive Linguistics: Current Applications and Future Perspectives (pp. 147-185). Berlin/New York Mouton de Gruyter.

Panther, K.-U., \& Radden, G. (1999). Metonymy in language and thought. Amsterdam ; Philadelphia: J. Benjamins.

Panther, K.-U., \& Radden, G. (1999). Metonymy in Language and Thought. Amsterdam/Philadelphia: John Benjamins.

Panther, K.-U., \& Thornburg, L. L. (1998). A Cognitive Approach to Inferencing in Conversation. Journal of Pragmatics(30), 755-769.

Panther, K.-U., \& Thornburg, L. L. (1999). The Potentiality for Actuality Metonymy in English and Hungarian. In K.-U. Panther \& G. Radden (Eds.), Metonymy in Language and Thought. Amsterdam/Philadelphia: John Benjamins.

Panther, K.-U., \& Thornburg, L. L. (2000). The EFFECT FOR CAUSE Metonymy in English Grammar. In A. Barcelona (Ed.), Metaphor and Metonymy at the Crossroads: A Cognitive Perspective. Berlin/New York: Mouton de Gruyter.

Panther, K.-U., \& Thornburg, L. L. (2002). The Role of Metaphor and Metonymy in English -er Nominals. In R. Dirven \& R. Pörings (Eds.), Metaphor and Metonymy in Comparison and Contrast (pp. 279-319). Berlin/New York: Mouton de Gruyter.

Panther, K.-U., \& Thornburg, L. L. (2003a). Metonymies as Natural Inference and Activation Schemas: The Case of Dependent Clauses as Independent Speech 
Acts. In K.-U. Panther \& L. L. Thornberg (Eds.), Metonymy and Pragmatic Inferencing (pp. 127-147). Amsterdam/Philadelphia: John Benjemins.

Panther, K.-U., \& Thornburg, L. L. (2003b). Metonymy and Pragmatic Inferencing. Philadelphia: John Benjamins.

Panther, K.-U., \& Thornburg, L. L. (2003c). On the Nature of Conceptual Metonymy. In K.-U. Panther \& L. L. Thornberg (Eds.), Metonymy and Pragmatic Inferencing (pp. 1-20). Amsterdam: John Benjamins.

Panther, K.-U., Thornburg, L. L., \& Barcelona, A. (Eds.). (2009). Metonymy and Metaphor in Grammar. Amsterdam: John Benjamins.

Paradis, C. (2004). Where does Metonymy Stop? Senses Facets, and Active Zones. Metaphor and symbol, 4(19), 245-264.

Pastra, K. (2009). Metonymy and Metaphor in Cross-Media Semantic Interplay: the COSMOROE Framework and Annotated Corpus. Paper presented at the RaAM 2009 International Workshop on Metaphor, Metonymy and Multimodality, Amsterdam.

Pecher, D., \& Zwaan, R. A. (Eds.). (2005). Grounding Cognition ---The Role of Perception and Action in Memory, Language, and Thinking. Cambridge: Cambridge University Press.

Peirsman, Y., \& Geeraerts, D. (2006). Metonymy as a Prototypical Category. Cognitive Linguistics, 17(3), 269-316.

Poulin-Dubois, D., Lepage, A., \& Ferland, D. (1996). Infants' Concept of Animacy. [doi: DOI: 10.1016/S0885-2014(96)90026-X]. Cognitive Development, 11(1), 19-36.

Pruyne, T. W. (2002). Sports Nicknames: 20,000 Professionals Worldwide. Jefferson, N.C: McFarland \& Co Inc.

Pustejovsky, J. (1998). The Generative Lexicon. MA: MIT

Quinn, P. C. (2011). Born to Categorize. In U. Goswami (Ed.), The Wiley-Blackwell handbook of Childhood Cognitive Development (2 ed., pp. 129-152). West Sussex: Wiley-Blackwell.

Quinn, P. C., Doran, M. M., Reiss, J. E., \& Hoffman, J. E. (2009). Time Course of Visual Attention in Infant Categorization of Cats Versus Dogs: Evidence for a Head Bias as Revealed Through Eye Tracking. Child Development, 80(1), 151-161. doi: 10.1111/j.1467-8624.2008.01251.x

Quinn, P. C., \& Eimas, P. D. (1996). Perceptual Cues That Permit Categorical Differentiation of Animal Species by Infants. Journal of Experimental Child Psychology, 63, 189-211.

Quinn, P. C., \& Eimas, P. D. (1998). Evidence for a Global Categorical Representation of Humans by Youg Infants. Journal of Experimental Child Psychology, 69, 151-174.

Quinn, P. C., \& Johnson, M. H. (2000). Global-Before-Basic Object Categorization in Connectionist Networks and 2-Month-Old Infants. Infancy, 1(1), 31-46.

Quinn, P. C., Yahr, J., Kuhn, A., Slater, A. M., \& Pascalis, O. (2002). Representation of the Gender of Human Faces by Infants: A Preference for Female. Perception, 31, 1109-1121.

Radden, G. (1992). The Cognitive Approach to Natural Language. In R. Dirven \& M. Putz (Eds.), Thirty Years of Linguistic Evolution: Studies in Honour of Rene Dirven on the Occasion of His Sixtieth Birthday (pp. 513-541). Armsterdam/Philadelphia: John Benjamins. 
Radden, G. (2000). How Metonymic are Metaphors? In A. Barcelona (Ed.), Metaphor and Metonymy at the Crossroads: A Cognitive Perspective (pp. 93109). Berlin/New York: Mouton de Gruyter.

Radden, G. (2002). How Metonymic are Metaphors? In R. Dirven \& R. Pörings (Eds.), Metaphor and Metonymy in Comparison and Contrast (pp. 407-434). Berlin/New York: Mouton de Gruyter.

Radden, G. (2005). The Ubiquity of Metonymy. In J. L. Otal, I. Navarro i Ferrando $\&$ B. Bellés Fortuno (Eds.), Cognitive and Discourse Approaches to Metaphor and Metonymy (pp. 11-28). Castello de la Plana: Publicacions de la Universitat Jaume I.

Radden, G. (2008). The Cognitive Approach to Language. In József Andor, Béla Hollósy, T. Laczkó \& Péter Pelyvás (Eds.), When Grammar Minds Language and Literature: Festschrift for Prof. Béla Korponay on the Occasion of his 80th Birthday (pp. 387-412). Debrecen: Institute of English and American Studies.

Radden, G., \& Dirven, R. (2007). Cognitive English Grammar. Amsterdam: John Benjamins.

Radden, G., Köpcke, K.-M., Berg, T., \& Siemund, P. (2007a). The Construction of Meaning in Language. In G. Radden, K.-M. Köpcke, T. Berg \& P. Siemund (Eds.), Aspects of Meaning Construction (pp. 1-15). Amsterdam/Philadelphia: John Benjamins.

Radden, G., Köpcke, K.-M., Berg, T., \& Siemund, P. (Eds.). (2007b). Aspects of Meaning Construction. Amsterdam/Philadelphia: John Benjamins.

Radden, G., \& Kövecses, Z. (1999). Towards a Theory of Metonymy In Klaus-Uwe Panther \& Günter Radden (Eds.), Metonymy in Language and Thought (pp. 17-59). Amsterdam/Philadelphia: John Benjamins.

Radden, G., \& Panther, K.-U. (Eds.). (2004). Studies in Linguistic Motivation. Berlin/New York: Mouton de Gruyter.

Rakison, D. H. (2003). Parts, Motion, and the Development of the AnimateInanimate Distinction in Infancy. In D. H. Rakison \& L. M. Oakes (Eds.), Early Category and Concept Development: Making Sense of the Blooming, Buzzing Confusion (pp. 159-192). Oxford: Oxford University Press.

Rakison, D. H. (2004). Infants' Sensitivity to Correlations between Static and Dynamic Features in A Category Context. Journal of Experimental Child Psychology, 89, 1-30.

Rakison, D. H., \& Butterworth, G. E. (1998). Infants' Use of Object Parts in Early Categorization. Developmental Psychology, 34(1), 49-62.

Rakison, D. H., \& Cohen, L. B. (1999). Infants' Use of Functional Parts in Basic-like Categorization. Developmental Science, 2(4), 423-431.

Rakison, D. H., \& Oakes, L. M. (Eds.). (2003). Early Category and Concept Development: Making Sense of the Blooming, Buzzing Confusion. Oxford: Oxford University Press.

Rakison, D. H., \& Poulin-Dubois, D. (2002). You Go This Way and I'll Go That Way: Developmental Changes in Infants' Detection of Correlations among Static and Dynamic Features in Motion Events. Child Development, 73(3), 682-699.

Rakison, D. H., \& Woodward, A. L. (2008). New Perspectives on the Effects of Action on Perceptual and Cognitive Development. Developmental Psychology, 44(5), 1209-1213. 
Rakison, D. H., \& Yermolayeva, Y. (2010). Infant Categorization. Wiley Interdisciplinary Reviews: Cognitive Science, 1(6), 894-905. doi: $10.1002 /$ wcs. 81

Reed, C. L., McGoldrick, J. E., Shackelford, R., \& Fidopiastis, C. (2004). Are Human Bodies Represented Differently From Other Animate and Inanimate Objects? Visual Cognition, 11, 523-550.

Reed, S. K. (2000). Problem Solving. In A. E. Kazdin (Ed.), Encyclopedia of Psychology (Vol. 8, pp. 71-75). Washington, DC: American Psychological Assocition and Oxford University Press.

Riad, S., \& Vaara, E. (2011). Varieties of National Metonymy in Media Accounts of International Mergers and Acuqisitions. Journal of Management Studies, 48(4), 737-771.

Riemer, N. (2002). When is a Metonymy no Longer a Metonymy? In R. Dirven \& R. Pörings (Eds.), Metaphor and Metonymy in Comparison and Contrast (pp. 379-406). Berlin/New York: Mouton de Gruyter.

Rohrer, T. (2005). Image Schemata in the Brain. In B. Hampe (Ed.), From Perception to Meaning: Image Schemas in Cognitive Linguistics (pp. 165196). Berlin: Walter de Gruyter.

Rohrer, T. (2007). Embodiment and Experientialism. In D. Geeraerts \& H. Cuyckens (Eds.), The Oxford Handbook of Cognitive Linguistics (pp. 25-47). Oxford: Oxford University Press.

Rosch, E. (1973). Natural Categories. Cognitive Psychology, 4, 328-350.

Rosch, E. (1975). Cognitive Reference Points. Cognitive Psychology, 7, 532-547.

Rosch, E., \& Lloyd, B. B. (Eds.). (1978). Cognition and Categorization Hillsdale, N.J. :: L. Erlbaum.

Rudzka-ostyn, B. (Ed.). (1988). Topics in Cognitive Linguistics. Amsterdam/Philadelphia: John Benjamins.

Ruiz de Mendoza, F. J. (1997). Cognitive and Pragmatic Aspects of Metonymy. Cuadernos de Filologia Inglesa(612), 161-178.

Ruiz de Mendoza, F. J. (1998). Understanding through Metonymy: The Role of Metonymy in Communication and Cognition. In B. Penas (Ed.), The Pragmatics of Understanding and Misunderstanding (pp. 197-208). Universidad de Zaragoza: Servicio de Publicaciones.

Ruiz de Mendoza, F. J. (2000). The Role of Mappings and Domains in Understanding Metonymy. In A. Barcelona (Ed.), Metaphor and Metonymy at the Crossroads: A Cognitive Perspective (pp. 109-132). Berlin/New York: Mouton de Gruyter.

Ruiz de Mendoza, F. J., \& Díez Velasco, O. I. (2002). Patterns of Conceptual Interaction. In R. Dirven \& R. Pörings (Eds.), Metaphor and Metonymy in Comparison and Contrast (pp. 489-532). Berlin/New York: Mouton de Gruyter.

Ruiz de Mendoza, F. J., \& Díez Velasco, O. I. (2003). High-level Action Metonymies in English and Spanish. Linguistics (Jezikoslovlje), 4(1), 121138.

Ruiz de Mendoza, F. J., \& Díez Velasco, O. I. (2003). High-level Metonymy and Linguistic Structure. In C. Inchaurralde \& C. Florén (Eds.), Interaction and Cognition in Linguistics (pp. 189-210). Frankfurt and New York: Peter Lang. Ruiz de Mendoza, F. J., \& Díez Velasco, O. I. (2004). Metonymic Motivation in Anaphoric Reference. In G. Radden \& K.-U. Panther (Eds.), Studies in Linguistic Motivation (pp. 293-320). Berlin/New York: Mouton de Gruyter. 
Ruiz de Mendoza, F. J., \& Otal Campo, J. L. (2002). Metonymy, Grammar, and Communication Granada: Comares.

Ruiz de Mendoza, F. J., \& Pérez Hernández, L. (2001). Metonymy and the Grammar: Motivation, Constraints and Interaction. Language \& Communication(21), 321-357.

Schreurs, B. H. J., Bakker, A. B., \& Schaufeli, W. B. (2009). Does Meaning-making Help during Organizational Change? Development and Validation of a New Scale. Career Development International, 14(6), 508-533.

Scott, P. H., Mortimer, E. F., \& Aguiar, O. G. (2006). The Tension Between Authoritative and Dialogic Discourse: A Fundamental Characteristic of Meaning Making Interactions in High School Science Lessons. Science Education, 90(4), 605-631.

Seoane, E., \& López-Couso, M. a. J. (Eds.). (2008). Theoretical and Empirical Issues in Grammaticalization Amsterdam/Philadelphia: John Benjamins.

Seto, K.-i. (1999). Distinguishing Metonymy from Synecdoche. In K.-U. Panther \& G. Radden (Eds.), Metonymy in Language and Thought (pp. 91-120). Amsterdam/Philadelphia: J. Benjamins.

Seto, K.-i. (2003). Metonymic Polysemy and Its Place in Meaning Extension. In B. Nerlich, Z. Todd, V. Herman \& D. D. Clarke (Eds.), Polysemy: Flexible Patterns of Meaning in Mind and Language (pp. 195-214). Berlin: Mouton de Gruyter.

Sharoff, S. (2006). Creating General-Purpose Corpora Using Automated Search Engine Queries. In M. Baroni \& S. Bernardini (Eds.), WaCky! Working Papers on the Web as Corpus (pp. 63-98). Bologna: GEDIT.

Sharoff, S. (2007). Classifying Web Corpora into Domain and Genre Using Automatic Feature Identification. Cahiers du Cental, 5, 1-10.

Shi, Y. (2006). Yu Fa Hua de Dong Yin Yu Ji Zhi (Motivation and Mechanism of Grammaticalization in Chinese). Beijing: Beijing University Press.

Shi, Y., \& Li, C. N. (2004). Han Yu Yu Fa Hua de Li Cheng: Xing Tai Ju Fa Fa Zhan de Dong Yin he Ji Zhi (A History of Grammaticalization in Chinese: Motivations and Mechanisms of Evolution of Chinese Morpho-syntax). Beijing: Beijing University Press.

Sinha, C., Silva Sinha, V. d., Zinken, J., \& Sampaio, W. (2011). When Time is not Space: The Social and Linguistic Construction of Time Intervals and Temporal Event Relations in an Amazonian Culture. Language and Cognition, 3(1), 137-169.

Slobin, D. I. (1994). Talking Perfectly: Discourse Origins of the Present Perfect. In W. Pagliuca (Ed.), Perspectives on Grammaticalization (pp. 119-133). Amsterdam/Philadelphia: John Benjamins.

Slobin, D. I. (1996). From "Thought and Language" to "Thinking for Speaking". In J. J. Gumperz \& S. C. Levinson (Eds.), Rethinking Linguistic Relativity (pp. 7096). Cambridge: Cambridge University Press.

Smith, L. B. (2005). Action Alters Shape Categories. Cognitive Science, 29, 665679.

Sperber, D., \& Wilson, D. (1995). Relevance: Communication and Cognition (Second ed.). Oxford: Blackwell.

Stallard, D. (1993). Two Kinds of Metonymy. Paper presented at the Annual Meeting of the ACL -- Proceedings of the 31st Annual Meeting on Association for Computational Linguistics, Columbus, Ohio. 
Stefanowitsch, A., \& Gries, S. T. (2006). Corpus-based Approaches to Metaphor and Metonymy. Berlin/New York: Mouton de Gruyter.

Stirling, L. (1996). Metonymy and Anaphora. Belgian Journal of Linguistics, 10, 6988.

Sugeno, T. (1996). Metonymy as the Primary Trope. Poetica, 46, 101-118.

Sun, C. (1996). Word-Order Change and Grammaticalization in the History of Chinese. California: Stanford University Press.

Sun, C. (2008). Two Conditions and Grammaticalization of the Chinese Locative. In D. Xu (Ed.), Space in Language of China: Cross-Linguistic Synchronic and Diachronic Perspectives (pp. 199-227). Paris: Springer Science + Business Media B. V.

Sweep, J. (2009). Metonymy without a Referential Shift. Linguistics in the Netherlands, 26(1), 103-114.

Taylor, J. R. (1995). Linguistic Categorizatin: Prototypes in Linguistic Theory (2nd ed.). Oxford: Clarendon Press.

Taylor, J. R. (2002a). Category Extension by Metonymy and Metaphor. In R. Dirven \& R. Pörings (Eds.), Metaphor and Metonymy in Comparison and Contrast (pp. 323-348). Berlin/New York: Mouton de Gruyter.

Taylor, J. R. (2002b). Cognitive Grammar. Oxford/New York: Oxford University Press.

Taylor, J. R. (2003). Linguistic Categorization. Oxford: Oxford University Press.

Taylor, J. R. (2006). Polysemy and the Lexicon. In G. Kristiansen, M. Achard, R. Dirven \& F. J. Ruiz de Mendoza Ibáñez (Eds.), Cognitive Linguistics: Current Applications and Future Perspectives (pp. 51-70). Berlin/New York: Mouton de Gruyter.

Thelen, E. (2000). Grounding in the World: Developmental Origins of the Embodied Mind. Infancy, 1(1), 3-28.

Thornburg, L. L., \& Panther, K.-U. (1997). Speech Act Metonymies. In W.-A. Liebert, G. Redeker \& L. Waugh (Eds.), Discourse and Perspective in Cognitive Linguistics (pp. 205-219). Amsterdam/Philadelphia: John Benjemins.

Tietze, S. (1998). The Role of Language in the Process of Creating Meaning in a Professional Organisation. PhD Phd, Sheffield Hallam University, Sheffield.

Ting, J., \& Chang, M. (2004). The Category of GEI in Mandarin Chinese and Grammaticalization. Taiwan Journal of Linguistics, 2(2), 45-74.

Tomasello, M. (2003). Constructing a Language. Cambridge: Harvard University Press.

Torralbo, A., Santiago, J., \& Lupiáñez, J. (2006). Flexible Conceptual Projection of Time onto Spatial Frames of Reference. Cognitive Science, 30, 745-757.

Träuble, B., \& Pauen, S. (2007). The Role of Functional Information for Infant Categorization. Cognition, 105, 362-379.

Traugott, E. C., \& Dasher, R. B. (2002). Regularity in Semantic Change. Cambridge: Cambridge University Press.

Traugott, E. C., \& König, E. (1991). The Semantics-Pragmatics of Grammaticalization Revisited. In E. C. Traugott \& B. Heine (Eds.), Approaches to Grammaticalization: Volume I. Theoretical and Methodological Issues (pp. 189-218). Amsterdam: John Benjamins.

Trenholm, S., \& Jensen, A. (2004). Interpersonal Communication (5 ed.). Oxford: Oxford University Press. 
Tsohatzidis, S. L. (1990a). Introduction. In S. L. Tsohatzidis (Ed.), Meanings and Prototypes-- Studies in Lingusitic Categorization (pp. 1-13). London/New York: Routledge.

Tsohatzidis, S. L. (Ed.). (1990b). Meanings and Prototypes-- Studies in Lingusitic Categorization. London/New York: Routledge.

Turati, C., Simion, F., \& Zanon, L. (2003). Newborns' Perceptual Categorization for Closed and Open Geometric Forms. Infancy, 4(3), 309-325. doi: 10.1207/s15327078in0403_01

Turner, M., \& Fauconnier, G. (2000). Metaphor, Metonymy, and Binding. In A. Barcelona (Ed.), Metaphor and Metonymy at the Crossroads: A Cognitive Perspective (pp. 133-145). Berlin/New York: Mouton de Gruyter.

Ullmann, S. (1962). Semantics: An Introduction to the Science of Meaning. Oxford: Basil Blackwell.

Ungerer, F., \& Schmid, H.-J. (2006). An Introduction to Cognitive Linguistics (2nd ed.). Harlow: Pearson/Longman.

Varela, F. J., Thompson, E., \& Rosch, E. (1991). The Embodied Mind: Cognitive Science and Human Experience Cambridge, Mass.: MIT Press.

Verhoeven, E., Skopeteas, S., Shin, Y.-M., Nishina, Y., \& Helmbrecht, J. (Eds.). (2008). Studies on Grammaticalization. Berlin/New York: Mouton de Gruyter.

Vine, B. (1999). Guide to The New Zealand Component of the International Corpus of English (ICE-NZ). Wellington: School of Linguistics and Applied Language Studies, Victoria University of Wellington.

Vygotsky, L. (1986). Thought and Language. Cambridge, Mass.: MIT Press.

Walkden, G. (2011). Abduction or Inertia? The Logic of Syntactic Change. In C. Cummins, C.-H. Elder, T. Godard, M. Macleod, E. Schmidt \& G. Walkden (Eds.), Proceedings of the Sixth Cambridge Postgraduate Conference in Language Research (pp. 230-239). Cambridge: Cambridge Institute of Language Research.

Wallace, D. (1999). The Impact of Email on Discursive Relations in the Workplace. Paper presented at the NZ Linguistics Society Conference, Massey University.

Wallace, D. (2000a). Email and the Problems of Communication. M/C: A Journal of Media and Culture, 3(4).

Wallace, D. (2000b). Positively Exploiting Email's Ambiguity. Employment Today, 38.

Wallace, D. (2001). Swimming in Separate Lanes: Rhetorical Practices in an Online Discussion Group. English in Aotearoa(43).

Wang, S.-h., Baillargeon, R., \& Brueckner, L. (2004). Young Infants' Reasoning about Hidden Objects: Evidence from Violation-of-Expectation Tasks with Test Trials only. Cognition, 93, 167-198.

Wang, S. H., Kaufman, L., \& Baillargeon, R. (2003). Should All Stationary Objects Move when Hit? Developments in Infants' Causal and Statistical Expectations about Collision Events. Infant Behavior and Development, 26(4), 529-567.

Warren, B. (1995). Distinguishing between Metaphor and Metonymy. In G.

Melchers \& B. Warren (Eds.), Studies in Anglistics (pp. 137-150). Stockholm: Almqvist \& Wilsell International.

Warren, B. (1998). What is Metonymy? In R. M. Hogg \& L. V. Bergen (Eds.), Historical Linguistics (Vol. 2 Germanic Linguistics, pp. 301-310). 
Warren, B. (1999). Aspects of Referential Metonymy. In K.-U. Panther \& G. Radden (Eds.), Metonymy in Language and Thought (pp. 121-135).

Amsterdam/Philadelphia: John Benjamins.

Warren, B. (2002). An Alternative Account of the Interpretation of Referential Metonymy and Metaphor. In R. Dirven \& R. Pörings (Eds.), Metaphor and Metonymy in Comparison and Contrast (pp. 113-133). Berlin/New York: Mouton de Gruyter.

Warren, B. (2004). Anaphoric Pronouns of Metonymic Expressions. Metaphorik.de(07/2004).

Warren, B. (2006). Referential metonymy. Stockholm: Almqvist \& Wiksell International.

Wilcox, P. (2004). A Cognitive Key: Metonymic and Metaphorical Mappings in ASL. Cognitive Linguistics, 15(2), 197-222.

Wilcox, T., \& Chapa, C. (2002). Infants' Reasoning about Opaque and Transparent Occluders in an Individuation Task. Cognition, 85, B1-B10.

Willems, R. M., \& Hagoort, P. (2007). Neural Evidence for the Interplay between Language, Gesture, and Action: A Review. Brain and Language, 101(3), 278-289.

Willems, R. M., Hagoort, P., \& Casasanto, D. (2010). Body-specific Representations of Action Verbs: Neural Evidence from Right-and Left-handers. Psychological Science, 21(1), 67-74.

Wilson, R. A. (2010). Meaning Making and the Mind of the Externalist. In R. Menary (Ed.), The Extended Mind (pp. 167-188). Cambridge, MA: MIT.

Wisher, I., \& Diewald, G. (Eds.). (2002). New Reflections on Grammaticalization. Amsterdam/Philadelphia: John Benjamins.

Yap, F.-h., Choi, P.-l., \& Cheung, K.-s. (2010). Delexicalizing di: How a Chinese Noun Has Evolved into an Attitudinal Nominalizer. In A. V. Linden, J.-C. Verstraete \& K. Davidse (Eds.), Formal Evidence in Grammaticalization Research (pp. 63-91). Amsterdam/Philadelphia: John Benjamins.

Younger, B. A., \& Fearing, D. D. (2000). A Global-to-Basic Trend in Early Categorization: Evidence From a Dual-Category Habituation Task. Infancy, 1(1), 47-58.

Zheng, D., \& Mai, M. (1964). Guhanyu Yufaxue Ziliao Huibian (A Collection of Materials on Old Chinese Grammar). Beijing: Zhonghua Shuju.

Ziemke, T. (2003). What's that Thing Called Embodiment? In Alterman \& Kirsh (Eds.), Proceedings of the 25th Annual Conference of the Cognitive Science Society (pp. 1134-1139). Mahwah, NJ: Lawrence Erlbaum.

Ziemke, T., Zlatev, J., \& Frank, R. M. (2007). Body, Language, and Mind - Volume 1: Embodiment Berlin/New York: Mouton de Gruyter. 